FABIO YUKIO TORIUMI

CONTROLE DIGITAL DE UMA PLANTA GIROSCÓPIO 
FABIO YUKIO TORIUMI

\title{
CONTROLE DIGITAL DE UMA PLANTA GIROSCÓPIO
}

\author{
Dissertação apresentada à Escola Po- \\ litécnica da Universidade de São Paulo para \\ obtenção do título de Mestre em Ciências.
}


FABIO YUKIO TORIUMI

\section{CONTROLE DIGITAL DE UMA PLANTA GIROSCÓPIO}

Dissertação apresentada à Escola Politécnica da Universidade de São Paulo para obtenção do título de Mestre em Ciências.

Área de Concentração:

Engenharia de Sistemas

Orientador:

Prof. Dr. Bruno Augusto Angélico 
Este exemplar foi revisado e corrigido em relação à versão original, sob responsabilidade única do autor e com a anuência de seu orientador.

São Paulo, de de

Assinatura do autor:

Assinatura do orientador:

Catalogação-na-publicação

Toriumi, Fabio Yukio

Controle Digital de uma Planta Giroscópio / F. Y. Toriumi -- versão corr. -São Paulo, 2016

$155 \mathrm{p}$.

Dissertação (Mestrado) - Escola Politécnica da Universidade de São Paulo. Departamento de Engenharia de Telecomunicações e Controle.

1.Controle digital 2.Sistemas dinâmicos 3.Controle (Teoria de Sistemas e Controle) I.Universidade de São Paulo. Escola Politécnica. Departamento de Engenharia de Telecomunicações e Controle II.t. 
Dedico à minha família e à minha namorada, que sempre me incentivaram e apoiaram em todos os momentos. 


\section{AGRADECIMENTOS}

Ao professor Dr. Bruno Augusto Angélico pela orientação, amizade e constante estímulo transmitido durante todo o período de mestrado.

Especialmente aos meus pais Mario Teruaki Toriumi e Elisa Akemi Abe Toriumi, e meu irmão Felipe Yoshio Toriumi, que sempre me incentivaram e me apoiaram desde o início do curso, e à minha namorada Elaine Naomi Watanabe, que me incentivou a iniciar o curso e esteve ao meu lado em todas as suas etapas.

Aos professores José Jaime da Cruz e Fabio de Oliveira Fialho pelo apoio nos tópicos desta dissertação, e aos meus amigos Gabriel Pereira das Neves e Fernando dos Santos Barbosa, do laboratório LCA (Laboratório de Controle Aplicado), pelo companheirismo e apoio durante todo o trabalho.

Ao LAC (Laboratório de Automação e Controle) da Escola Politécnica da USP pela disponibilização dos recursos físicos.

Aos meus familiares, amigos e todos que colaboraram, diretamente ou indiretamente, na execução desta dissertação.

Ao CNPq (Conselho Nacional de Desenvolvimento Científico e Tecnológico) pela bolsa de mestrado concedida com o número de processo 161464/2014-9. 


\section{RESUMO}

Esta dissertação apresenta a modelagem matemática de um giroscópio de controle de momento $(\mathrm{CMG})$ e aplica técnicas de controle digital para o controle de atitude dessa planta. CMGs são atuadores importantes para o controle de atitude de corpos no espaço, tais como satélites e veículos espaciais. Desenvolve-se a modelagem da planta por meio da mecânica Lagrangiana e estudam-se técnicas específicas de controle PID (proporcionalintegral-derivativo) e LQR (linear-quadratic regulator) digitais para aplicação em sistemas com a planta nas configurações desacoplada e acopladas de fase mínima e não-mínima. Realizam-se ensaios via simulação, com o modelo não-linear da planta, bem como via experimentos práticos, com o sistema real, para validar os controladores projetados. Com isso, obtêm-se resultados positivos com ambas as técnicas estudadas, com exceção ao caso acoplado de fase não-mínima, para o qual uma das técnicas não foi capaz de controlar a atitude da planta.

Palavras-Chave - Controle digital. Sistemas dinâmicos. Controle (Teoria de Sistemas e Controle). Giroscópio CMG. Controle aplicado. 


\section{ABSTRACT}

This dissertation presents the mathematical modeling of a control moment gyroscope $(\mathrm{CMG})$ and applies digital control techniques for its attitude control. CMGs are important actuators for bodies attitude control in the space, such as satellites and space vehicles. The system modeling is developed with the Lagrangian mechanics and a digital proportionalintegral-derivative (PID) and a digital linear-quadratic regulator (LQR) control techniques are studied and applied to a decoupled system and coupled systems with minimum and non-minimum phases. Simulation tests with the non-linear plant model and experimental tests with the real plant are done in order to validate the control techniques. Then, positive results are reached with both studied techniques, excepting in the coupled system with non-minimum phase, in which one technique was not able to control it.

Keywords - Digital control. Dynamic systems. Control (Systems and Control Theory). CMG gyroscope. Control Application. 


\section{LISTA DE FIGURAS}

1 Sistema experimental . . . . . . . . . . . . . . . . 22

2 Descrição da planta giroscópio CMG modelo 750 . . . . . . . . . . . . . . . 24

3 Sistema de coordenadas dos corpos da planta . . . . . . . . . . . . 26

4 Diagrama de blocos considerado para o projeto dos controladores . . . . . 37

$5 \quad$ Diagrama de blocos do sistema com controle LQR e integradores . . . . . . 39

6 Diagrama de blocos da planta com realimentação de velocidade em tempo

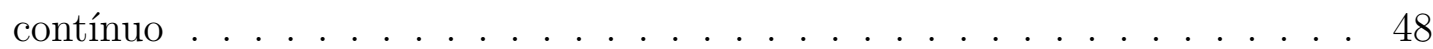

7 Diagrama de blocos da planta com realimentação de velocidade em tempo discreto . . . . . . . . . . . . . . . . . . . 49

8 Lugar das raízes do controlador PID f . . . . . . . . . . . . . . . 52

$9 \quad$ Estrutura do diagrama de blocos dos arquivos Simulink . . . . . . . . . . 55

10 Gráficos com os sinais $r(t)$ dos ensaios considerados para o teste de acompanhamento de referência . . . . . . . . . . . . . . . . . 58

11 Gráficos com os sinais $d(t)$ dos ensaios considerados para o teste de rejeição à perturbação na saída da planta . . . . . . . . . . . . . . . . . . . . . 58

12 Gráficos com a visão ampliada dos sinais de saída obtidos na região inicial do primeiro pulso retangular aplicado nos experimentos dos testes de acompanhamento de referência com pulsos retangulares simultâneos (ensaio 2) - Sistemas de fase mínima (desacoplado e acoplado) . . . . . . . . . . . . . 61

13 Gráficos com os sinais de erros de acompanhamento de referência obtidos nos experimentos dos testes de acompanhamento de referência com sinais senoidais (ensaio 3) - Sistemas de fase mínima (desacoplado e acoplado) . .

14 Gráficos com a visão ampliada dos sinais de saída obtidos na região inicial do primeiro pulso retangular aplicado nos experimentos dos testes de rejeição à perturbação na saída da planta com pulsos retangulares simultâneos (ensaio 2) - Sistemas de fase mínima (desacoplado e acoplado) 64 
15 Gráficos com a visão ampliada na região de regime permanente dos sinais de saída obtidos nos experimentos dos testes de rejeição à perturbação com sinais senoidais (ensaio 3) - Sistemas de fase mínima (desacoplado e acoplado) 65

16 Gráficos com os resultados do teste de acompanhamento de referência com sinais do ensaio 3 com amplitude de $40^{\circ}$ - Sistema desacoplado com controle

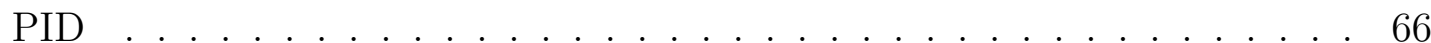

17 Gráficos com a visão ampliada em cada região dos pulsos retangulares aplicados nos experimentos dos testes de acompanhamento de referência com pulsos retangulares simultâneos (ensaio 2) - Sistema acoplado de fase não-mínima com o controle LQR . . . . . . . . . . . . . . . . . . 68

18 Etapas da fixação dos sistemas de coordenadas aos corpos da planta . . . . 74

19 Análise dos ângulos $\theta_{1}$ e $\psi_{1}$ para o cálculo de ${ }_{0}^{1} R \ldots$. . . . . . . . . . 78

20 Análise dos ângulos $\theta_{2}$ e $\psi_{2}$ para o cálculo de ${ }_{1}^{2} R \ldots$. . . . . . . . . . . 79

21 Análise dos ângulos $\theta_{3}$ e $\psi_{3}$ para o cálculo de ${ }_{2}^{3} R \ldots$. . . . . . . . . . . 81

22 Análise dos ângulos $\theta_{4}$ e $\psi_{4}$ para o cálculo de ${ }_{3}^{4} R \ldots$. . . . . . . . . . . . 82

23 Gráficos com os sinais obtidos no procedimento Nutation: Frequency \& Mode Shapes . . . . . . . . . . . . . . . . . . . 87

24 Gráficos com os sinais obtidos no procedimento Precession . . . . . . . . . 89

25 Configurações da planta para os procedimentos Inertia Tests . . . . . . . . 91

26 Gráficos com os sinais das respostas obtidas nos procedimentos Inertia Tests 92

27 Gráficos com os sinais obtidos nos procedimentos Control Effort Gain Tests 96

28 Gráficos com os resultados do teste de acompanhamento de referência com sinais do ensaio 1 (positivo) - Sistema desacoplado com controle PID . . . 101

29 Gráficos com os resultados do teste de acompanhamento de referência com sinais do ensaio 1 (negativo) - Sistema desacoplado com controle PID . . . 102

30 Gráficos com os resultados do teste de rejeição à perturbação com sinais do ensaio 1 (positivo) - Sistema desacoplado com controle PID . . . . . . . 103

31 Gráficos com os resultados do teste de rejeição à perturbação com sinais do ensaio 1 (negativo) - Sistema desacoplado com controle PID . . . . . . . 104 
32 Gráficos com os resultados do teste de acompanhamento de referência com sinais do ensaio 2 - Sistema desacoplado com controle PID . . . . . . . . . 105

33 Gráficos com os resultados do teste de rejeição à perturbação com sinais do ensaio 2 - Sistema desacoplado com controle PID . . . . . . . . . . . . . 106

34 Gráficos com os resultados do teste de acompanhamento de referência com sinais do ensaio 3 - Sistema desacoplado com controle PID . . . . . . . . . 107

35 Gráficos com os resultados do teste de rejeição à perturbação com sinais do ensaio 3 - Sistema desacoplado com controle PID . . . . . . . . . . . . . 108

36 Gráficos com os resultados do teste de acompanhamento de referência com sinais do ensaio 1 (positivo) - Sistema desacoplado com controle LQR . . . 110

37 Gráficos com os resultados do teste de acompanhamento de referência com sinais do ensaio 1 (negativo) - Sistema desacoplado com controle LQR . . . 111

38 Gráficos com os resultados do teste de rejeição à perturbação com sinais do ensaio 1 (positivo) - Sistema desacoplado com controle LQR . . . . . . 112

39 Gráficos com os resultados do teste de rejeição à perturbação com sinais do ensaio 1 (negativo) - Sistema desacoplado com controle LQR . . . . . . 113

40 Gráficos com os resultados do teste de acompanhamento de referência com sinais do ensaio 2 - Sistema desacoplado com controle LQR . . . . . . . . . 114

41 Gráficos com os resultados do teste de rejeição à perturbação com sinais do ensaio 2 - Sistema desacoplado com controle LQR . . . . . . . . . . . . 115

42 Gráficos com os resultados do teste de acompanhamento de referência com sinais do ensaio 3 - Sistema desacoplado com controle LQR . . . . . . . . . 116

43 Gráficos com os resultados do teste de rejeição à perturbação com sinais do ensaio 3 - Sistema desacoplado com controle LQR . . . . . . . . . . . . 117

44 Gráficos com os resultados do teste de acompanhamento de referência com sinais do ensaio 1 (positivo) - Sistema acoplado de fase mínima com controle PID

45 Gráficos com os resultados do teste de acompanhamento de referência com sinais do ensaio 1 (negativo) - Sistema acoplado de fase mínima com con- 
46 Gráficos com os resultados do teste de rejeição à perturbação com sinais do ensaio 1 (positivo) - Sistema acoplado de fase mínima com controle PID 121

47 Gráficos com os resultados do teste de rejeição à perturbação com sinais do ensaio 1 (negativo) - Sistema acoplado de fase mínima com controle PID 122

48 Gráficos com os resultados do teste de acompanhamento de referência com sinais do ensaio 2 - Sistema acoplado de fase mínima com controle PID . . 123

49 Gráficos com os resultados do teste de rejeição à perturbação com sinais do ensaio 2 - Sistema acoplado de fase mínima com controle PID . . . . . . 124

50 Gráficos com os resultados do teste de acompanhamento de referência com sinais do ensaio 3 - Sistema acoplado de fase mínima com controle PID . . 125

51 Gráficos com os resultados do teste de rejeição à perturbação com sinais do ensaio 3 - Sistema acoplado de fase mínima com controle PID . . . . . . 126

52 Gráficos com os resultados do teste de acompanhamento de referência com sinais do ensaio 1 (positivo) - Sistema acoplado de fase mínima com controle LQR . . . . . . . . . . . . . . . . . . . . . 128

53 Gráficos com os resultados do teste de acompanhamento de referência com sinais do ensaio 1 (negativo) - Sistema acoplado de fase mínima com controle LQR . . . . . . . . . . . . . . . . . . . . . . . . . . . . 12

54 Gráficos com os resultados do teste de rejeição à perturbação com sinais do ensaio 1 (positivo) - Sistema acoplado de fase mínima com controle LQR 130

55 Gráficos com os resultados do teste de rejeição à perturbação com sinais do ensaio 1 (negativo) - Sistema acoplado de fase mínima com controle LQR131

56 Gráficos com os resultados do teste de acompanhamento de referência com sinais do ensaio 2 - Sistema acoplado de fase mínima com controle LQR . . 132

57 Gráficos com os resultados do teste de rejeição à perturbação com sinais do ensaio 2 - Sistema acoplado de fase mínima com controle LQR . . . . . 133

58 Gráficos com os resultados do teste de acompanhamento de referência com sinais do ensaio 3 - Sistema acoplado de fase mínima com controle LQR . . 134

59 Gráficos com os resultados do teste de rejeição à perturbação com sinais do ensaio 3 - Sistema acoplado de fase mínima com controle LQR . . . . . 135 
60 Gráficos com os resultados do teste de acompanhamento de referência com sinais do ensaio 2 - Sistema acoplado de fase não-mínima com controle LQR137

61 Diagrama de blocos do Simulink para a simulação do sistema com o controlador PID . . . . . . . . . . . . . . . . . . . . . 139

62 Diagrama de blocos do Simulink para o experimento do sistema com o controlador PID . . . . . . . . . . . . . . . . . . . . . . 140

63 Diagrama de blocos do Simulink para a simulação do sistema com o controlador LQR . . . . . . . . . . . . . . . . . . . . . . . . . . . . 141

64 Diagrama de blocos do Simulink para o experimento do sistema com o controlador LQR . . . . . . . . . . . . . . . . . . . . . . . . . . . 142

65 Subsistemas com o diagrama de blocos da planta, utilizado para a simulação (a) e para o experimento (b) do sistema com o controlador PID . 143

66 Subsistemas com o diagrama de blocos do controlador PID (a), da realimentação de velocidade da planta realizada para o controle PID (b), e do controle PI simples de velocidade do disco. . . . . . . . . . . . . . . . . . . 144

67 Algoritmo do bloco Matlab Function com as equações discretas do controlador LQR . . . . . . . . . . . . . . . . . . . . . . . . . . . . . . 144 


\section{LISTA DE TABELAS}

1 Valores numéricos dos parâmetros da planta . . . . . . . . . . . . . . . . 29

2 Dados de desempenho dos resultados obtidos na região inicial do primeiro pulso retangular dos testes de acompanhamento de referência com pulsos retangulares simultâneos (ensaio 2) - Sistemas de fase mínima (desacoplado

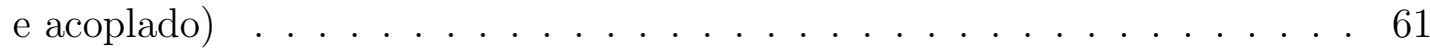

3 Dados de desempenho dos resultados obtidos na região inicial do primeiro pulso retangular dos testes de rejeição à perturbação na saída da planta com pulsos retangulares simultâneos (ensaio 2) - Sistemas de fase mínima (desacoplado e acoplado) . . . . . . . . . . . . . . . . . . . 63

4 Dados de desempenho dos resultados obtidos em cada região dos pulsos retangulares aplicados no teste de acompanhamento de referência com pulsos retangulares simultâneos (ensaio 2) - Sistema acoplado de fase não-mínima com o controle LQR . . . . . . . . . . . . . . . . . . . . . . . 67

5 Momentos de inércia fornecidos pela ECP . . . . . . . . . . . . . . 90

6 Velocidades obtidas nos procedimentos Inertial Tests . . . . . . . . . . . . 93

7 Momentos de inércia calculados . . . . . . . . . . . . . . . . . 93

8 Valores obtidos no procedimento Control Effort Gain Test \#2 . . . . . . . 97

9 Ganhos dos atuadores calculados . . . . . . . . . . . . . . . . 97

10 Ganhos dos encoders calculados . . . . . . . . . . . . . . . . . 98 


\section{SUMÁRIO}

1 Introdução $\quad 16$

1.1 Revisão Bibliográfica . . . . . . . . . . . . . . . . . . . . 16

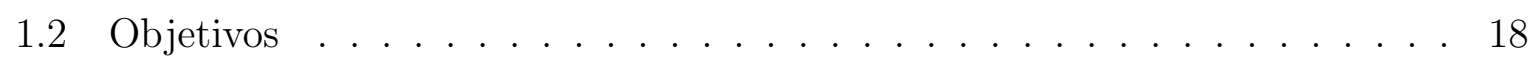

1.2 .1 Objetivo Geral . . . . . . . . . . . . . . . 18

1.2 .2 Objetivos Específicos . . . . . . . . . . . . . 18

1.3 Justificativa . . . . . . . . . . . . . . . . . . . . . . . . . 18

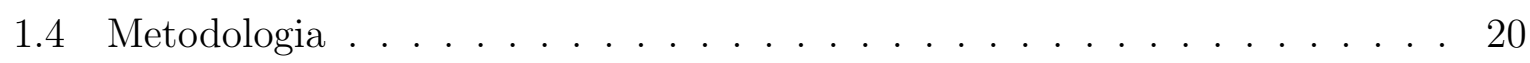

1.5 Artigos publicados . . . . . . . . . . . . . . 21

2 Modelagem Matemática da Planta CMG 22

2.1 Descrição do Sistema Experimental . . . . . . . . . . . . . . . . 22

2.2 Sistemas de Coordenadas dos Corpos . . . . . . . . . . . . . . 24

2.3 Equações não-lineares da Planta . . . . . . . . . . . . . . . . . 25

2.4 Equações linearizadas da Planta . . . . . . . . . . . . . . . . . . 27

2.5 Parâmetros do Giroscópio . . . . . . . . . . . . . . . . . 28

2.6 Modelos Lineares Multivariáveis da Planta . . . . . . . . . . . . . . . . . 29

2.6.1 Modelo Desacoplado da Planta (Caso Desacoplado de Fase Mínima) 29

2.6.2 Modelo Acoplado da Planta . . . . . . . . . . . . . . . . . 33

2.6.2.1 Caso Acoplado de Fase Mínima . . . . . . . . . . . . 33

2.6.2.2 Caso Acoplado de Fase Não-Mínima . . . . . . . . . . . 35

3 Estratégias de Controle $\quad 37$

3.1 Controle PID Digital . . . . . . . . . . . . . . . . . 37

3.2 Controle LQR Digital com Ação Integrativa . . . . . . . . . . . . . . . . 39 
3.2 .1 Inserção dos Integradores . . . . . . . . . . . . . . . . . . . . . . . . 40

3.2.2 Estratégia de Controle LQR Digital . . . . . . . . . . . . . . . . . . 41

3.2.2.1 Controle Ótimo Quadrático (COQ) . . . . . . . . . . . 42

3.2.2.2 Regulador Linear Quadrático (LQR) . . . . . . . . . . 43

4 Projetos dos Controladores $\quad 47$

4.1 Projeto do Controlador PID Digital . . . . . . . . . . . . . . . . . 47

4.1 .1 Realimentação de Velocidade na Planta . . . . . . . . . . . . . . . . 47

4.1.2 Projeto do Controlador . . . . . . . . . . . . . . . . . . . 50

4.2 Projeto do Controlador LQR Digital . . . . . . . . . . . . . . . . 53

5 Procedimento Experimental $\quad 55$

6 Análise e Discussão dos Resultados $\quad 59$

6.1 Casos de Fase Mínima (Desacoplado e Acoplado) . . . . . . . . . . . . . . 59

6.2 Caso Acoplado de Fase Não-Mínima . . . . . . . . . . . . . . . . . . . . 67

7 Conclusões e Sugestões de Trabalhos Futuros $\quad 69$

$\begin{array}{ll}\text { Referências } & 71\end{array}$

Apêndice A - Fixação dos Sistemas de Coordenadas aos Corpos $\quad 73$

$\begin{array}{ll}\text { Apêndice B - Vetores de Velocidade Angular } & 75\end{array}$

$\begin{array}{ll}\text { Apêndice C - Matrizes de Rotação } & 77\end{array}$

Apêndice D - Matrizes de Inércia Central dos Corpos $\quad 83$

Apêndice E - Equações Não-Lineares $\quad 84$

Apêndice F - Modelo Não-Linear $\quad 85$ 
Apêndice I - Diagrama de Blocos criados no Simulink 


\section{INTRODUÇÃO}

É possível reconhecer três categorias diferentes de giroscópios em relação ao aspecto construtivo (ARMENISE et al., 2010): vibratórios, ópticos e mecânicos.

Os giroscópios vibratórios utilizam elementos ressonantes, tais como materiais piezoelétricos, para detectar a variação na orientação espacial de um corpo. Por outro lado, os giroscópios ópticos utilizam bobinas de fibras ópticas para detectar variações na orientação, com base na alteração do padrão de interferência. Estes dois tipos são utilizados como sensores inerciais.

Um giroscópio mecânico pode ser definido, de modo geral, como um corpo sólido capaz de rotacionar em altas velocidades angulares em relação a um eixo que passa em um ponto fixo, podendo ou não ser o centro de gravidade do corpo (SCARBOROUGH, 1958). Este tipo de giroscópio possui uma configuração básica que explora as propriedades inerciais de um disco rotacionando em alta velocidade, o qual tende a manter a direção de seu eixo de rotação devido à tendência de um corpo a resistir a mudanças de direção de seu momento. Este tipo de giroscópio pode ser utilizado como um sensor inercial ou como um atuador. Um atuador deste tipo muito conhecido é o CMG (Control Moment Gyroscope ou Giroscópio de Controle de Momento), que é muito utilizado para estabilização e orientação de satélites e veículos espaciais. Sua estrutura é composta por um disco e por um, ou mais, gimbals ${ }^{1}$ motorizados que mudam a direção do momento angular do disco. Conforme o disco é inclinado, ocorre uma mudança de orientação do seu momento angular, o que gera um torque giroscópico em sua estrutura. CMGs vêm sendo utilizados por décadas em grandes veículos espaciais, como o Skylab, o Mir Space Station e o International Space Station.

\subsection{Revisão Bibliográfica}

Os giroscópios em geral podem ser utilizados tanto como sensores como atuadores. Existem artigos na literatura que exploram a aplicação do giroscópio como um atuador, como em Brown e Xu (1997) que utiliza um giroscópio acoplado dentro de uma estru-

\footnotetext{
${ }^{1}$ Estruturas geralmente em formato de arco.
} 
tura em forma de roda, para efetuar a sua direção e estabilidade explorando as forças e reações giroscópicas, possibilitando a locomoção em altas velocidades, tanto em terrenos irregulares como em aplicações anfíbias. Em Townsend e Shenoi (2014) dois giroscópios são utilizados para minimizar o balanço de um navio causado pelas ondas do mar. Em Gagne et al. (2012) um CMG é posicionado na estrutura de um equipamento, utilizado em cirurgias cardíacas, para minimizar o deslocamento do epicárdio (superfície do coração), no qual o cirurgião opera diretamente em um procedimento coronary artery bypass graft (CABG). Em Yetkin et al. (2014) um CMG é utilizado na estrutura de uma bicicleta para equilibrá-la verticalmente em repouso. Em Bhat e Tiwari (2009) são analisados diferentes arranjos de estruturas com diversas quantidades de CMGs para o controle de atitude ${ }^{2}$ de um veículo espacial. E, por fim, em Yavuzoglu et al. (2011) é feita uma análise do uso de CMG para o controle de atitude de um satélite AGILE por meio de simulações e testes; sendo nestes quatro últimos explorado o uso de CMGs. Diferentes técnicas de controle foram aplicadas nestes artigos para atingir seus objetivos.

Nesta dissertação utiliza-se uma planta giroscópio CMG da fabricante ECP, modelo 750. Diferentes técnicas de controle aplicadas nessa planta podem ser encontradas em Reyhanoglu e Loo (2006), Durand et al. (2014), Abbas et al. (2013) e Mardan et al. (2015), entre outros.

Em Reyhanoglu e Loo (2006) um controle por realimentação de estados é aplicado por meio de uma abordagem de passos retroativos em cascada (cascaded back-stepping) para o controle do deslocamento angular de dois corpos específicos da planta em uma configuração restrita (com um grau de liberdade a menos). Em Durand et al. (2014) um controle LQR (Linear-Quadratic Regulator), com atualização do ganho de realimentação baseado em eventos, é aplicado em tempo contínuo para o controle de atitude da planta por meio de ação integrativa. Em Abbas et al. (2013) um controle por realimentação de estados com uma estratégia LPV (linear parameter-varying) é aplicado em tempo contínuo para o controle de atitude da planta por meio de pré filtros nas entradas de referência. E por fim em Mardan et al. (2015) um controle $H_{2} / H_{\infty}$ baseado em LMI (Linear Matrix Inequalities) é aplicado em tempo contínuo para o controle de atitude da planta; sendo nestes três últimos explorado o controle de atitude da planta.

\footnotetext{
${ }^{2}$ Controle da orientação de um objeto em relação a um sistema inercial de referência.
} 


\subsection{Objetivos}

Os objetivos desta dissertação são divididos entre objetivo geral e objetivos específicos.

\subsubsection{Objetivo Geral}

Efetuar a modelagem e o controle de atitude de uma planta giroscópio CMG por meio de técnicas de controle digital.

\subsubsection{Objetivos Específicos}

- Estudar o comportamento e as principais características da planta;

- Determinar os valores numéricos dos parâmetros da planta;

- Obter um modelo não-linear e modelos lineares, em tempo discreto, da planta;

- Estudar técnicas de controle digital;

- Projetar controladores digitais com base nas técnicas de controle estudadas e nos modelos lineares em tempo discreto;

- Executar simulações, com o modelo não-linear obtido, e experimentos práticos, com a planta física, para cada controlador projetado;

- Analisar e comparar os resultados obtidos.

\subsection{Justificativa}

A abordagem do controle na forma digital é explorada nesta dissertação devido à grande tendência atual da aplicação deste tipo de controle em relação ao controle analógico. Isso se deve, principalmente, à disponibilidade de hardwares digitais de baixo custo e às vantagens em trabalhar com sinais digitais em relação a sinais de tempo contínuo, conforme apontado no Capítulo 1 em Ogata (1995). Tal texto enfatiza a grande versatilidade do controlador digital, no qual pode ser utilizada uma gama maior de leis de controle em relação ao controlador analógico, além de executar, rapidamente, operações computacionais complexas com precisão, ao contrário do controlador analógico que exige um alto custo conforme o aumento da complexidade das operações. 
No Capítulo 12 em Chen (1995) (p. 478), também são enfatizadas as vantagens que sistemas digitais possuem em relação a sistemas analógicos, argumentando que sistemas digitais são mais precisos, mais confiáveis, mais flexíveis e geralmente mais baratos que os sistemas analógicos, além de mostrar que sinais digitais são menos suscetíveis a ruídos e oscilações da rede de energia, concluindo que frequentemente é mais desejável a realização digital de controladores.

Um controlador digital pode ser projetado em tempo contínuo e, em seguida, discretizado, ou projetado diretamente em tempo discreto. A primeira abordagem tem como principais vantagens o fato de ser mais intuitivo e a enorme quantidade de técnicas de projeto presentes na literatura técnica. No entanto, se o efeito do atraso devido às conversões A/D (Analógico para Digital) e D/A (Digital para Analógico) não for considerado no projeto, o desempenho do sistema de controle pode ser degradado. Tal efeito é minimizado com aumento da frequência de amostragem. Em contrapartida, elevadas frequências de amostragem podem resultar em problemas de aproximação numérica, principalmente em hardwares com aritmética em ponto fixo.

Por outro lado, o projeto diretamente no plano- $z$ já considera os efeitos "nocivos" da amostragem. Tem como desvantagem o fato de ser menos intuitivo e não possuir uma literatura tão vasta como no caso contínuo.

Conforme visto na Seção 1.1, o CMG é utilizado como atuador em diversas aplicações; algumas em modo regulatório, no qual não são inseridos sinais de referência para o posicionamento da estrutura, ou seja, é feito apenas o posicionamento em torno de um ponto de operação, como em Gagne et al. (2012) e Yetkin et al. (2014); e outras em modo de rastreamento, nas quais são inseridos sinais de referência para a orientação da estrutura, como em Bhat e Tiwari (2009) e Yavuzoglu et al. (2011). O modo de rastreamento é muito utilizado e explorado para o controle de atitude de CMGs, como pode ser visto em Durand et al. (2014), Abbas et al. (2013) e Mardan et al. (2015); que utilizam a mesma planta CMG, mas aplicam diferentes técnicas de controle.

Com base no exposto acima, as justificativas para esta dissertação são listadas:

- O controle digital é muito versátil;

- Sistemas embarcados com algoritmos de controle digital são essenciais para o desenvolvimento tecnológico;

- O projeto de controladores em tempo discreto já considera os efeitos das conversões $\mathrm{A} / \mathrm{D}$ e $\mathrm{D} / \mathrm{A}$; 
- Boa parte dos sistemas de controle de atitude de satélites e veículos espaciais são embarcados e utilizam o CMG como atuadores.

\subsection{Metodologia}

Para alcançar os objetivos desta dissertação, obtém-se primeiramente o modelo matemático não-linear da planta giroscópio CMG, por meio da mecânica Lagrangiana, que por sua vez é utilizada para derivar o modelo matemático linearizado, em torno de um ponto de operação. Feito isso, obtêm-se os valores numéricos de alguns parâmetros da planta física por meio dos procedimentos descritos no manual do giroscópio (ECP, 1999).

Em seguida, definem-se três pontos de operação específicos, nos quais um gera um modelo desacoplado de fase mínima da planta e, os outros dois, geram modelos acoplados, uma de fase mínima e outra de fase não-mínima. Após serem descritos em forma de espaço de estados, em tempo contínuo, calculam-se os seus equivalentes em tempo discreto, os quais serão utilizados como modelos lineares para os projetos dos controladores digitais.

Na etapa do projeto do controle digital, são estudadas algumas técnicas específicas (estratégias) de controle PID (proporcional-integral-derivativo) digital e LQR (LinearQuadratic Regulator) digital, sendo que, para o projeto do controlador PID digital, considerase uma realimentação de velocidade na planta para amortecer a sua ressonância de nutação, característica comentada na Seção F.1, e para o projeto do controlador LQR digital, considera-se uma inserção de integradores na malha de controle.

Por conseguinte, validam-se os controladores projetados via simulações, com o modelo não-linear em tempo contínuo da planta, e de experimentos práticos, com a planta física. Os resultados obtidos são analisados e comparados. 


\subsection{Artigos publicados}

Durante o desenvolvimento desta dissertação, alguns artigos foram gerados, diretamente ou indiretamente, pelos temas estudados e desenvolvidos. Alguns deles, que foram aceitos para publicação, são:

- o artigo "Geometric Modeling and Robust Control of a Gyroscopic System" foi apresentado na "27th Conference on Mechanical Vibration and Noise" da "ASME (The American Society of Mechanical Engineers)", em Agosto de 2015, Boston, Massachusetts, USA. Esta dissertação contribuiu, neste artigo, com a parte da modelagem da planta giroscópio CMG;

- o artigo "State Feedback Decoupling Control of a Control Moment Gyroscope" foi aprovado pela revista "Journal of Control, Automation and Electrical Systems", e foi publicado online, com número DOI 10.1007/s40313-016-0277-8, em Setembro de 2016. Esta dissertação contribuiu, neste artigo, com a parte da modelagem da planta giroscópio CMG;

- o artigo "Controle de Atitude Digital Aplicado a uma Planta Giroscópio" apresentado no "XXI Congresso Brasileiro de Automática" em Outubro de 2016, Vitória, Espirito Santo, Brasil. Este artigo foi gerado durante o desenvolvimento desta dissertação, com os resultados obtidos nos experimentos práticos realizados com os sistemas compostos pela planta na configuração desacoplada de fase mínima e com os controladores PID ou LQR digitais projetados nesta dissertação. Há apenas algumas diferenças neste artigo, que são pequenas variações obtidas nos valores calculados dos momentos de inércia dos corpos, e nos valores adotados para as amplitudes e durações do pulso retangular no procedimento experimental. 


\section{MODELAGEM MATEMÁTICA DA PLANTA CMG}

Neste capítulo, descreve-se a planta giroscópio CMG (Control Moment Gyroscope) utilizada e sua modelagem matemática é desenvolvida. O manual que acompanha o equipamento (ECP, 1999) utiliza o método de Kane para realizar a modelagem, porém de forma bem resumida e sem apresentar as etapas de seu desenvolvimento. Portanto, optase por refazer a modelagem utilizando a mecânica Lagrangiana e fixando os sistemas de coordenadas aos corpos da planta por meio de uma outra convenção, diferente da adotada no manual.

\subsection{Descrição do Sistema Experimental}

O sistema experimental utilizado nesta dissertação é composto por três subsistemas conforme a Figura 1: Computador Pessoal, Caixa de Comando e a Planta Giroscópio CMG.

Figura 1: Sistema experimental

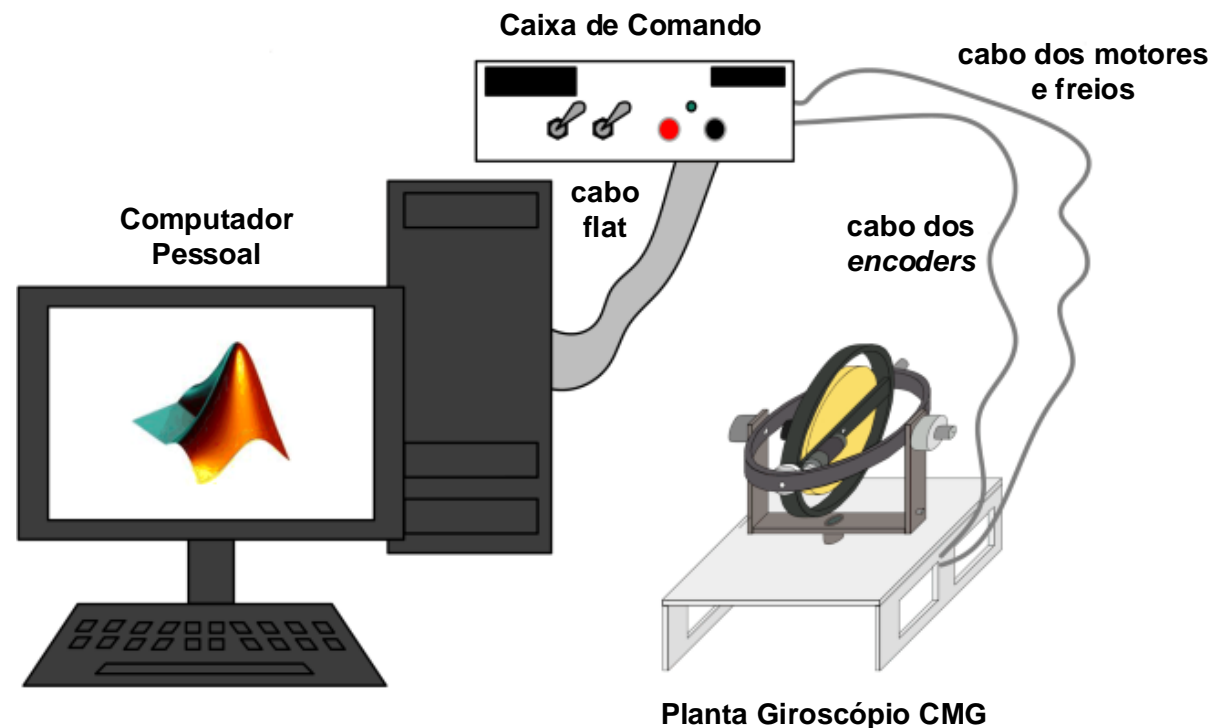

Fonte: Autor.

O Computador Pessoal é utilizado para a execução das simulações e experimentos práticos por meio do programa MATLAB ${ }^{\circledR} /$ Simulink. 
A Caixa de Comando da fabricante ECP é composta pelo amplificador de potência e pelos comandos manuais localizados em seu painel: dois botões para ligar e desligar o aparelho e duas chaves interruptoras para acionar os freios. Ela é conectada a uma placa de processamento digital de sinais, instalada no barramento PCI do computador pessoal, via cabo flat.

A Planta Giroscópio CMG da fabricante ECP, modelo 750, possui uma estrutura versátil com 4 graus de liberdade, na qual podem ser aplicados até dois sinais de entrada simultâneos (torques de dois motores), e são gerados quatro sinais de saída (posições angulares de quatro corpos). Ele permite limitar a quantidade de graus de liberdade de maneira a se obter desde sistemas SISO para controle linear simples, com apenas um grau de liberdade, até sistemas MIMO para controle não-linear, com quatro graus de liberdade.

Essa planta eletromecânica, ilustrada na Figura 2, é composta por quatro corpos rígidos rotacionais $(A, B, C$ e $D)$, possuindo cada um uma posição angular $\psi_{n}(n=$ 1,2,3,4), respectivamente, em torno de seu eixo de rotação \#n. Um disco axissimétrico de bronze (corpo $D$ ) é rotacionado em torno do eixo \#4 por meio de um motor DC (motor \#4), responsável por fornecer o torque $T_{m_{4}}$. O encoder incremental $D$ é responsável por medir a posição angular $\psi_{4}$ do corpo $D$, em relação ao corpo $C$. O gimbal interno (corpo $C$ ) é rotacionado em torno do eixo \#3 por intermédio de outro motor DC (motor \#3), responsável por fornecer o torque $T_{m_{3}}$. A posição angular $\psi_{3}$ do corpo $C$, em relação ao corpo $B$, é medida pelo encoder incremental $C$. Outro gimbal externo (corpo $B$ ) rotaciona em torno do eixo \#2, no qual não é aplicado nenhum torque externo, e a posição angular $\psi_{2}$ do corpo $B$, em relação ao corpo $A$, é medida pelo encoder incremental $B$. Da mesma forma, uma base giratória (corpo $A$ ) rotaciona em torno do eixo \#1 também sem a ação de um torque externo. A posição angular $\psi_{1}$ do corpo $A$, em relação à base fixa da planta, é medida pelo encoder incremental $A$.

Os eixos \#1 e \#2 possuem freios, que podem ser acionados manualmente por meio de chaves interruptoras no painel da caixa de comando, para impedir o deslocamento angular dos corpos $A$ e $B$, respectivamente. Há também sensores inerciais (inertial switches) instalados nos corpos $A, B$ e $C$ que detectam qualquer condição de velocidade acima do limite nos eixos \#1, \#2 e \#3, respectivamente. Quando algum desses sensores detecta uma condição de alta variação angular, a caixa de comando é desligada, acionando, assim, os freios dos eixos \#1 e \#2. Dessa forma, esses eixos são desacelerados e parados toda vez que uma condição de alta velocidade angular for detectada. 
Figura 2: Descrição da planta giroscópio CMG modelo 750

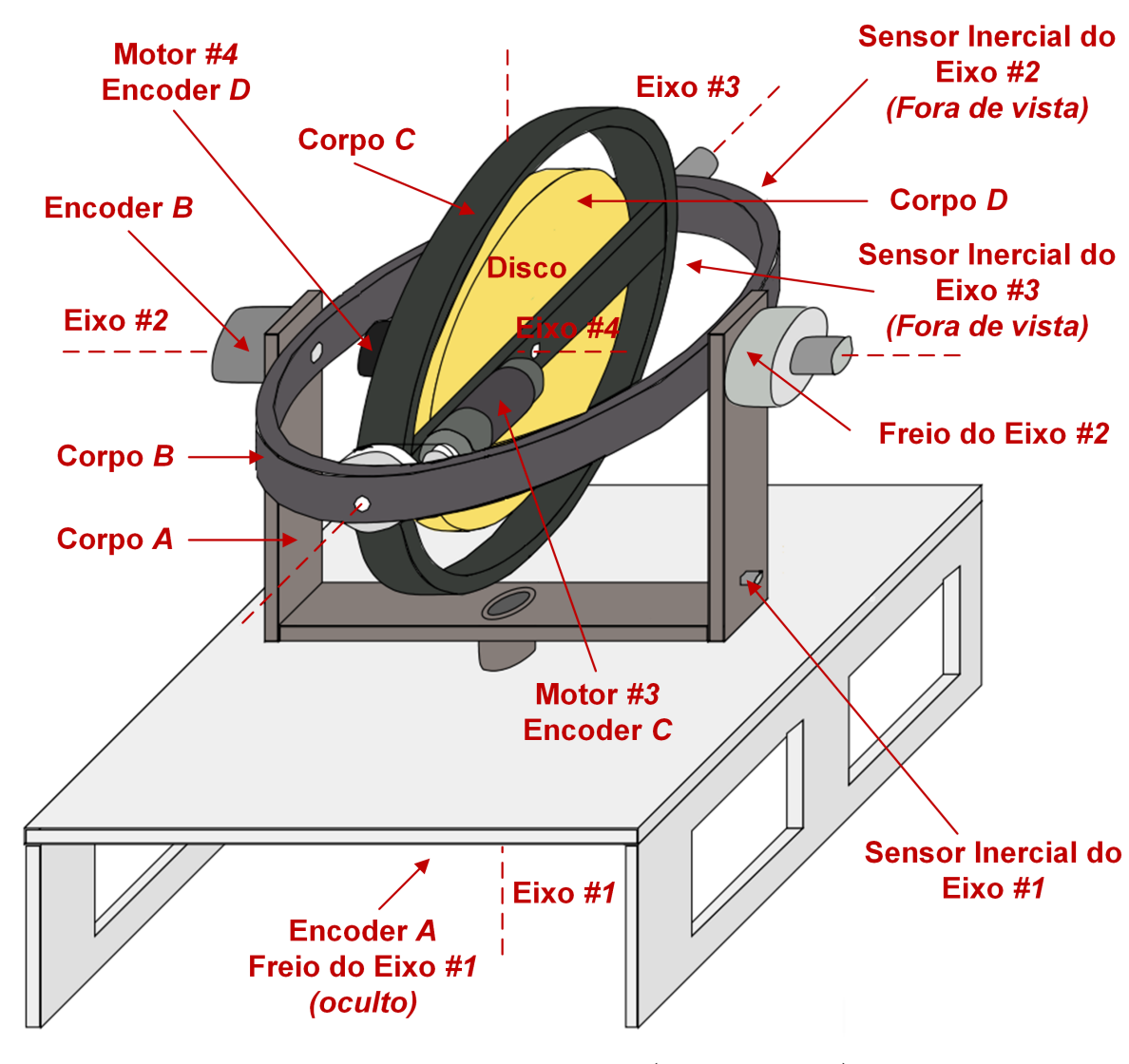

Fonte: adaptado de (ECP, 1999).

Como a estrutura montada do giroscópio é toda simétrica, a origem do centro de massa de cada corpo está localizada sempre no centro do disco (corpo $D$ ), onde também serão adotadas as origens de todos os sistemas de coordenadas dos corpos, para que apenas a dinâmica rotacional da planta seja considerada nos cálculos de sua modelagem, possibilitando que as matrizes de inércia sejam simplificadas e consideradas como diagonais, o que minimiza os efeitos da gravidade.

\subsection{Sistemas de Coordenadas dos Corpos}

Os sistemas de coordenadas, definidas com a notação $\{n\}(n=1,2,3,4)$, são fixadas aos corpos rígidos $A, B, C$ e $D$ da planta, respectivamente, por meio da convenção definida na Seção 3.4 em Craig (2005), com o objetivo de determinar a posição e a orientação do efetuador com base nas variáveis das juntas da planta (ângulos, no caso de juntas rotacionais, ou deslocamentos lineares, no caso de juntas prismáticas). No caso da planta CMG, há apenas juntas rotacionais (não há deslocamento linear dos corpos) e, por 
analogia, o seu efetuador é o disco ${ }^{1}$.

A descrição detalhada desse procedimento de fixação dos sistemas de coordenadas aos corpos rígidos da planta CMG é apresentada no Apêndice A, onde define-se o ângulo formado entre os versores $\hat{X}_{n}$ e $\hat{X}_{n-1} \operatorname{como} \theta_{n}$.

Porém, para o processo de modelagem da planta, são definidos os ângulos $\psi_{n}$ como sendo as posições angulares dos corpos. Uma vez que os encoders são do tipo incremental, elas devem ser zeradas no inicio de cada experimento, obedecendo o seguinte posicionamento inicial de cada corpo $\left(\psi_{n}=0\right)$ :

- O ângulo $\psi_{1}$ pode ser zerado em qualquer posição inicial da base giratória (corpo $A)$, pois isso não afeta a dinâmica do giroscópio;

- O ângulo $\psi_{2}$ deve ser zerado posicionando o gimbal externo (corpo $B$ ) perpendicularmente à base giratória (corpo $A)$;

- O ângulo $\psi_{3}$ deve ser zerado posicionando o gimbal interno (corpo $C$ ) perpendicularmente ao gimbal externo (corpo $B)$;

- E por fim, o ângulo $\psi_{4}$ pode ser zerado em qualquer posição do disco (corpo $\left.D\right)$, uma vez que sua posição angular não afeta diretamente a dinâmica do giroscópio.

Essa orientação do giroscópio é denominado de "configuração de reset" e nela há uma diferença entre $\theta_{n}$ e $\psi_{n}$ somente para $n=2$, onde os versores $\hat{X}_{2}$ e $\hat{X}_{1}$ formam um ângulo de $90^{\circ}$ entre si $\left(\psi_{2}=\theta_{2}+90^{\circ}\right)$, como pode ser observado na Seção C.2. Para os outros valores de $n$, os versores $\hat{X}_{n}$ e $\hat{X}_{n-1}$ estão sobrepostos um ao outro, ou seja, $\psi_{n}=\theta_{n}$.

Todos os sistemas de coordenadas resultantes possuem suas origens fixadas no centro do disco (Corpo D), por definição. Porém, para facilitar a visualização do resultado, exibido na Figura 3, eles foram deslocados de suas origens, com exceção de $\{4\}$.

\subsection{Equações não-lineares da Planta}

As equações não-lineares da dinâmica da planta são obtidas pela Formulação Dinâmica de Lagrange (Lagrangian Dynamic Formulation), descrita na Seção 6.9 em Craig (2005):

$$
\tau_{n_{e x t}}-\frac{\mathrm{d}}{\mathrm{d} t} \frac{\partial L}{\partial \dot{\psi}_{n}}+\frac{\partial L}{\partial \psi_{n}}=0
$$

\footnotetext{
${ }^{1}$ Fisicamente, a planta CMG não possui um efetuador, mas o último sistema de coordenadas a ser fixado nela é o do disco (corpo D), como pode ser visto no Apêndice A.
} 
Figura 3: Sistema de coordenadas dos corpos da planta

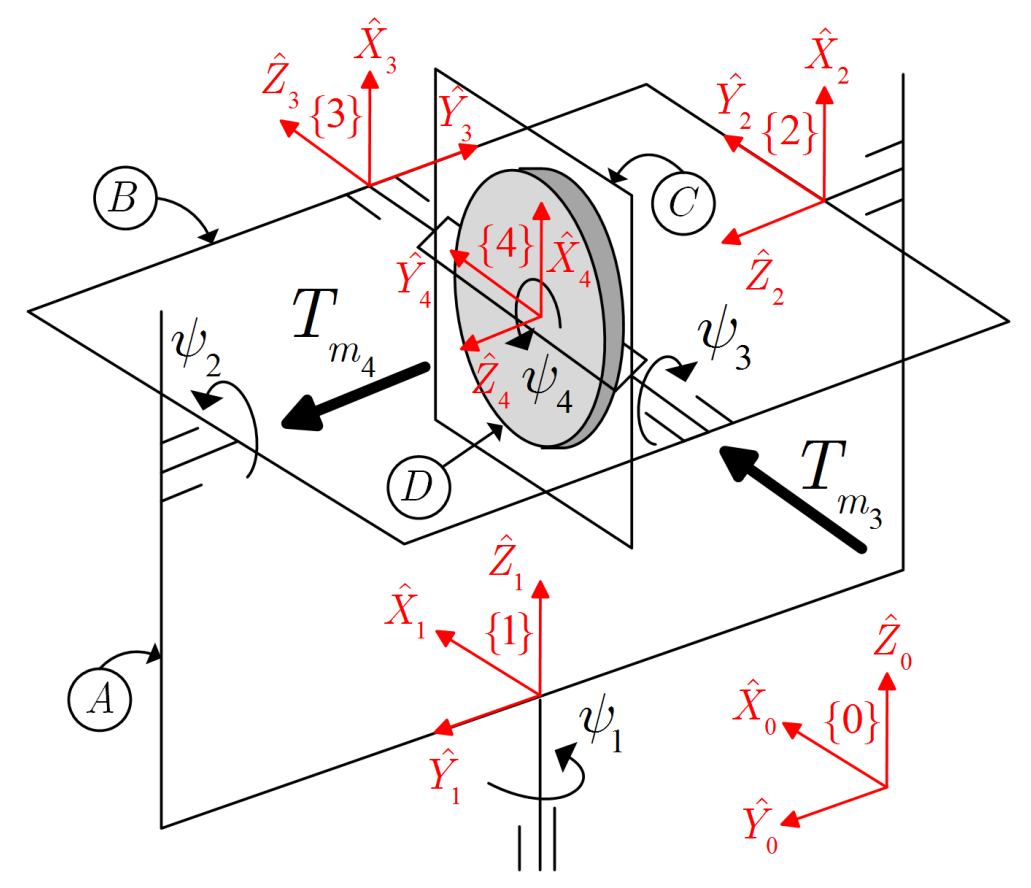

Fonte: Autor.

sendo $L$ a função escalar denominada Lagrangeano, $\tau_{n_{e x t}}$ os torques externos aplicados nos eixos $\# n, \dot{\psi}_{n}=\omega_{n}$ e $n=1,2,3,4$.

Como as origens dos centros de massa dos corpos e do sistema de coordenadas inercial $\{0\}$ estão localizadas sempre no centro do disco (corpo $D$ ), o Lagrangeano é composto somente pela energia cinética da planta:

$$
L=K=\sum_{n=1}^{4}\left[\frac{1}{2} \cdot m_{n} \cdot V_{c_{n}}^{T} \cdot V_{c_{n}}+\frac{1}{2} \cdot{ }^{n} \omega_{n}{ }^{T} \cdot\left(I_{n} \cdot{ }^{n} \omega_{n}\right)\right],
$$

sendo:

- $m_{n}$ as massas dos corpos rígidos atrelados aos sistemas de coordenadas $\{n\}$ (Corpos $A, B, C$ e $D$ são atrelados aos sistemas de coordenadas $\{1\},\{2\},\{3\}$ e $\{4\}$, respectivamente);

- $V_{c_{n}}$ as velocidades dos centros de massa dos corpos rígidos atrelados a $\{n\}$ em relação ao sistema inercial $\{0\}$;

- ${ }^{n} \omega_{n}$ os vetores de velocidade angular dos corpos rígidos em relação aos seus próprios sistemas de coordenadas $\{n\}$, calculados e apresentados em (B.2) a (B.5) no Apêndice B;

- $I_{n}$ as matrizes de inércia central dos corpos rígidos atrelados aos sistemas de coordenadas $\{n\}$, apresentadas em (D.1) no Apêndice D. 
Porém, como os corpos rígidos do giroscópio não se deslocam linearmente, ou seja, $V_{c_{n}}=0$. O Lagrangeano resultante é:

$$
L=K=\sum_{n=1}^{4}\left[\frac{1}{2} \cdot{ }^{n} \omega_{n}{ }^{T} \cdot\left(I_{n} \cdot{ }^{n} \omega_{n}\right)\right] .
$$

Calculando $(2.3)^{2}$, resulta em:

$$
\begin{aligned}
L= & \frac{I_{y y_{D}}}{2} \omega_{4}^{2}+\frac{\left(I_{x x_{C}}+I_{x x_{D}}\right)}{2} \omega_{3}^{2}+\frac{\left[I_{y y_{B}}+I_{y y_{C}}+I_{y y_{D}}+\left(I_{z z_{C}}+I_{x x_{D}}-I_{y y_{C}}-I_{y y_{D}}\right) \sin ^{2}\left(\psi_{3}\right)\right]}{2} \omega_{2}^{2}+ \\
& \frac{\left[I_{z z_{A}}+I_{z z_{B}}+I_{z z_{C}}+I_{x x_{D}}+\left(I_{x x_{B}}+I_{x x_{C}}-I_{z z_{B}}-I_{z z_{C}}\right) \sin ^{2}\left(\psi_{2}\right)+\left(I_{y y_{C}}+I_{y y_{D}}-I_{z z_{C}}-I_{x x_{D}}\right) \cos ^{2}\left(\psi_{2}\right) \sin ^{2}\left(\psi_{3}\right)\right]}{2} \omega_{1}^{2}+ \\
& I_{y y_{D}} \cos \left(\psi_{3}\right) \omega_{2} \omega_{4}+I_{y y_{D}} \cos \left(\psi_{2}\right) \sin \left(\psi_{3}\right) \omega_{1} \omega_{4}-\left(I_{x x_{C}}+I_{x x_{D}}\right) \sin \left(\psi_{2}\right) \omega_{1} \omega_{3}+ \\
& \left(I_{y y_{C}}+I_{y y_{D}}-I_{z z_{C}}-I_{x x_{D}}\right) \cos \left(\psi_{2}\right) \cos \left(\psi_{3}\right) \sin \left(\psi_{3}\right) \omega_{1} \omega_{2} .
\end{aligned}
$$

Ao resolver (2.1) ${ }^{3}$ para $n=1,2,3,4$, geram-se as equações não-lineares da planta, descritas em (E.1) a (E.4) no Apêndice E, que possuem a forma:

$$
\begin{aligned}
T_{m_{4}}+ & f_{4}\left(\psi_{2}, \psi_{3} ; \omega_{1}, \omega_{2}, \omega_{3} ; \dot{\omega}_{1}, \dot{\omega}_{2}, \dot{\omega}_{4}\right)=0, \\
T_{m_{3}}+ & f_{3}\left(\psi_{2}, \psi_{3} ; \omega_{1}, \omega_{2}, \omega_{4} ; \dot{\omega}_{1}, \dot{\omega}_{3}\right)=0 \\
& f_{2}\left(\psi_{2}, \psi_{3} ; \omega_{1}, \omega_{2}, \omega_{3}, \omega_{4} ; \dot{\omega}_{1}, \dot{\omega}_{2}, \dot{\omega}_{4}\right)=0, \\
& f_{1}\left(\psi_{2}, \psi_{3} ; \omega_{1}, \omega_{2}, \omega_{3}, \omega_{4} ; \dot{\omega}_{1}, \dot{\omega}_{2}, \dot{\omega}_{3}, \dot{\omega}_{4}\right)=0
\end{aligned}
$$

sendo que as funções $f_{4}, f_{3}, f_{2}$ e $f_{1}$ não dependem das posições angulares $\psi_{1}$ e $\psi_{4}$, pois o comportamento dinâmico do sistema é idêntico em quaisquer posições iniciais arbitrárias da base giratória e do disco.

Vale ressaltar que algumas dinâmicas não foram consideradas na modelagem, como o atrito nas juntas da planta. O modelo não-linear resultante ${ }^{4}$, que é utilizado nas simulações desta dissertação, é descrito no Apêndice F, onde também é descrito o procedimento utilizado para a sua validação.

\subsection{Equações linearizadas da Planta}

As equações linearizadas da planta são geradas nesta dissertação por meio da Expansão em Série de Taylor das equações não-lineares (E.1) à (E.4), na qual são utilizados os dois primeiros termos da expansão (ordens 0 e 1), em torno do seguinte ponto de

\footnotetext{
${ }^{2}$ com auxílio da ferramenta symbolic toolbox do programa MATLAB ${ }^{\circledR}$.

${ }^{3}$ lembrando que os torques externos $\tau_{4_{e x t}}$ e $\tau_{3_{e x t}}$ são, respectivamente, os torques aplicados pelos motores \#4 $\left(T_{m_{4}}\right)$ e \#3 $\left(T_{m_{3}}\right)$ e os torques externos $\tau_{2_{e x t}}$ e $\tau_{1_{e x t}}$ são nulos.

${ }^{4}$ composto pela solução das equações não-lineares (E.1) a (E.4) explicitamente para as variáveis de aceleração angular $\dot{\omega}_{n}$.
} 
operação, que é de equilíbrio estável, definido como:

$$
\begin{gathered}
\dot{\omega}_{1}=\dot{\omega}_{2}=\dot{\omega}_{3}=\dot{\omega}_{4}=0 \mathrm{rad} / \mathrm{s}^{2} ; \quad \omega_{1}=\omega_{2}=\omega_{3}=0 \mathrm{rad} / \mathrm{s} ; \quad \psi_{1}=\psi_{4}=0 \mathrm{rad} ; \\
\omega_{4}=\Omega(\mathrm{em} \mathrm{rad} / \mathrm{s}), \quad \psi_{2}=\psi_{2_{0}}(\mathrm{em} \mathrm{rad}), \quad \psi_{3}=\psi_{3_{0}}(\mathrm{em} \mathrm{rad}) .
\end{gathered}
$$

Considerando pequenas variações em torno desse ponto de operação, as equações linearizadas da planta são calculadas, resultando nas seguintes equações:

$$
\begin{aligned}
& T_{m_{4}}-I_{y y_{D}} \dot{\omega}_{4}-I_{y y_{D}} \cos \left(\psi_{3_{o}}\right) \dot{\omega}_{2}-I_{y y_{D}} \sin \left(\psi_{3_{o}}\right) \cos \left(\psi_{2_{o}}\right) \dot{\omega}_{1}=0, \\
& T_{m_{3}}-\left(I_{x x_{C}}+I_{x x_{D}}\right) \dot{\omega}_{3}+\left(I_{x x_{C}}+I_{x x_{D}}\right) \sin \left(\psi_{2_{o}}\right) \dot{\omega}_{1}-I_{y y_{D}} \sin \left(\psi_{3_{o}}\right) \Omega \omega_{2}+ \\
& I_{y y_{D}} \cos \left(\psi_{3_{o}}\right) \cos \left(\psi_{2_{o}}\right) \Omega \omega_{1}=0 \\
& -I_{y y_{D}} \cos \left(\psi_{3_{o}}\right) \dot{\omega}_{4}-\left(I_{y y_{B}}+I_{y y_{C}}+I_{y y_{D}}\right) \dot{\omega}_{2}+\left(I_{y y_{C}}+I_{y y_{D}}-I_{z z_{C}}-I_{x x_{D}}\right) \sin ^{2}\left(\psi_{3_{o}}\right) \dot{\omega}_{2}- \\
& \left(I_{y y_{C}}+I_{y y_{D}}-I_{z z_{C}}-I_{x x_{D}}\right) \cos \left(\psi_{2_{o}}\right) \cos \left(\psi_{3_{o}}\right) \sin \left(\psi_{3_{o}}\right) \dot{\omega}_{1}+I_{y y_{D}} \sin \left(\psi_{3_{o}}\right) \Omega \omega_{3}- \\
& I_{y y_{D}} \sin \left(\psi_{3_{o}}\right) \sin \left(\psi_{2_{o}}\right) \Omega \omega_{1}=0, \\
& -I_{y y_{D}} \sin \left(\psi_{3_{o}}\right) \cos \left(\psi_{2_{o}}\right) \dot{\omega}_{4}+\left(I_{x x_{C}}+I_{x x_{D}}\right) \sin \left(\psi_{2_{o}}\right) \dot{\omega}_{3}+ \\
& \left(I_{z z_{C}}+I_{x x_{D}}-I_{y y_{C}}-I_{y y_{D}}\right) \cos \left(\psi_{2_{o}}\right) \cos \left(\psi_{3_{o}}\right) \sin \left(\psi_{3_{o}}\right) \dot{\omega}_{2}-\left(I_{z z_{A}}+I_{z z_{B}}+I_{z z_{C}}+I_{x x_{D}}\right) \dot{\omega}_{1}+ \\
& \left(I_{z z_{B}}+I_{z z_{C}}-I_{x x_{B}}-I_{x x_{C}}\right) \sin ^{2}\left(\psi_{2_{o}}\right) \dot{\omega}_{1}+\left(I_{z z_{C}}+I_{x x_{D}}-I_{y y_{C}}-I_{y y_{D}}\right) \sin ^{2}\left(\psi_{3_{o}}\right) \cos ^{2}\left(\psi_{2_{o}}\right) \dot{\omega}_{1}- \\
& I_{y y_{D}} \cos \left(\psi_{3_{o}}\right) \cos \left(\psi_{2_{o}}\right) \Omega \omega_{3}+I_{y y_{D}} \sin \left(\psi_{3_{o}}\right) \sin \left(\psi_{2_{o}}\right) \Omega \omega_{2}=0 .
\end{aligned}
$$

\subsection{Parâmetros do Giroscópio}

A planta CMG, modelo 750 da fabricante ECP, possui alguns parâmetros físicos, como os momentos de inércia dos corpos e os ganhos dos encoders e dos atuadores. Seus valores numéricos são necessários para a simulação numérica do modelo da planta, sendo alguns fornecidos pela fabricante em seu manual (ECP, 1999) e outros obtidos ao executar procedimentos específicos, detalhados também no manual. Os detalhes da execução destes procedimentos são apresentados no Apêndice G e os valores obtidos estão descritos na Tabela 1. Neste apêndice, os valores dos momentos de inércia dos corpos são calculados em Kg.m² na Seção G.1, os ganhos dos atuadores são calculados em N.m/counts ${ }^{5}$ na Seção G.2, e os ganhos dos encoders incrementais em counts/rad ${ }^{6}$ na Seção G.3.

\footnotetext{
${ }^{5}$ essa unidade counts se refere ao valor digital do conversor D/A de 16-bit, limitado entre $-2^{15}$ a $2^{15}$. ${ }^{6}$ essa outra unidade counts se refere aos pulsos de encoder, pois os encoders da planta são incrementais.
} 
Tabela 1: Valores numéricos dos parâmetros da planta

\begin{tabular}{cccc}
\hline Parâmetro & Símbolo & Valor & Unidade \\
\hline & $I_{z z_{A}}$ & 0,0698 & \\
Momentos & $I_{x x_{B}}$ & 0,0119 & \\
de & $I_{y y_{B}}$ & 0,0178 & \\
Inércia & $I_{z z_{B}}$ & 0,0297 & \\
dos & $I_{x x_{C}}$ & 0,0124 & Kg.m ${ }^{2}$ \\
Corpos & $I_{y y_{C}}$ & 0,0278 & \\
$(A, B, C, D)$ & $I_{z z_{C}}$ & 0,0188 & \\
& $I_{x_{D}}$ & 0,0148 & \\
& $I_{y y_{D}}$ & 0,0273 & \\
\hline Ganho & $k_{e_{A}}$ & 81504 & \\
dos & $k_{e_{B}}$ & 81504 & counts \\
Encoders & $k_{e_{C}}$ & 124256 & rad \\
& $k_{e_{D}}$ & 33952 & \\
\hline Ganho & $k_{m_{4}}$ & $2,1544 \cdot 10^{-5}$ & N.m \\
dos Atuadores & $k_{m_{3}}$ & $8,9355 \cdot 10^{-5}$ & counts \\
\hline
\end{tabular}

Fonte: Autor e (ECP, 1999).

\subsection{Modelos Lineares Multivariáveis da Planta}

Nesta dissertação, são explorados três casos com configurações diferentes da planta: um caso desacoplado de fase mínima e dois casos acoplados, uma de fase mínima e outra de fase não-mínima.

\subsubsection{Modelo Desacoplado da Planta (Caso Desacoplado de Fase Mínima)}

A planta possui algumas configurações que geram modelos lineares multivariáveis desacoplados. Os pontos de operação (2.9) dessas configurações são definidos em:

$$
\Omega \neq 0 \mathrm{rad} / \mathrm{s} \text { e } \psi_{2_{0}}=c t e .(\mathrm{em} \mathrm{rad}) \text { e } \psi_{3_{0}}=0 \mathrm{rad}
$$

sendo $0<\Omega \leq 800 \mathrm{RPM}(\approx 83,776 \mathrm{rad} / \mathrm{s})$ e $0 \leq\left|\psi_{2_{0}}\right|<90^{\circ}(\approx 1,571 \mathrm{rad})$.

Independentemente do valor de $\Omega$ escolhido (dentro do intervalo definido acima), para valores de $\psi_{2_{0}}$ negativos ou nulo, geram-se modelos desacoplados de fase mínima, e para valores de $\psi_{2_{0}}$ positivos, geram-se modelos desacoplados de fase não-mínima.

O ponto de operação da configuração desacoplada considerado nesta dissertação é: $\Omega=400 \mathrm{RPM} \approx 41,888 \mathrm{rad} / \mathrm{s} ; \psi_{2_{0}}=0^{\circ}=0 \mathrm{rad} ; \mathrm{e} \psi_{3_{0}}=0^{\circ}=0 \mathrm{rad}$. 
Ao avaliar as equações linearizadas (2.10) a (2.13) neste ponto de operação, considerando os valores numéricos dos momentos de inércia dos corpos, descritos na Tabela 1 , obtêm-se respectivamente:

$$
\begin{aligned}
T_{m_{4}}-0,0273 \dot{\omega}_{4}-0,0273 \dot{\omega}_{2} & =0, \\
T_{m_{3}}-0,0272 \dot{\omega}_{3}+1,1435 \omega_{1} & =0, \\
0,0273 \dot{\omega}_{4}+0,0729 \dot{\omega}_{2} & =0, \\
0,1331 \dot{\omega}_{1}+1,1435 \omega_{3} & =0,
\end{aligned}
$$

chegando-se às seguintes equações:

$$
\begin{aligned}
& \dot{\omega}_{3}=42,0419 \omega_{1}+36,7647 T_{m_{3}}, \\
& \dot{\omega}_{2}=-21,9298 T_{m_{4}}, \\
& \dot{\omega}_{1}=-8,5916 \omega_{3} .
\end{aligned}
$$

Neste ponto, os sinais de controle enviados para os motores $\# h(h=4,3)$ estão em unidades do conversor D/A (counts) ${ }^{7}$. Para obter os torques aplicados $T_{m_{h}}$ pelos motores, em N.m, eles devem ser multiplicados pelos ganhos dos atuadores $k_{m_{h}}$. Assim como as velocidades $\omega_{n}(n=1,2,3,4)$, que estão em unidades de pulsos de encoder (counts), devem ser divididas pelos ganhos $k_{e_{m}}(m=A, B, C, D)$, respectivamente, para serem processadas $\mathrm{em} \mathrm{rad} / \mathrm{s}$.

Dessa forma, ao considerar esses ganhos em (2.19) a (2.21) tem-se que:

$$
\begin{aligned}
& \dot{\omega}_{3}=\left(42,0419 \frac{\omega_{1}}{k_{e_{1}}}+36,7647 T_{m_{3}} k_{m_{3}}\right) k_{e_{3}}=64,0945 \omega_{1}+408,1968 T_{m_{3}}, \\
& \dot{\omega}_{2}=-21,9298 T_{m_{4}} \cdot k_{m_{4}} \cdot k_{e_{2}}=-38,5074 T_{m_{4}}, \\
& \dot{\omega}_{1}=-8,5916 \omega_{3} \cdot \frac{k_{e_{1}}}{k_{e_{3}}}=-5,6355 \omega_{3} .
\end{aligned}
$$

As Equações (2.22) a (2.24), escritas na forma de representação em espaço de estados, geram o seguinte modelo em tempo contínuo:

$$
\left\{\begin{array}{l}
\dot{x}(t)=A x(t)+B u(t) \\
y(t)=C x(t)
\end{array}\right.
$$

\footnotetext{
${ }^{7} \mathrm{Na}$ caixa de comando, há uma malha interna de controle de corrente, cuja dinâmica é muito mais rápida que a dinâmica da planta e, portanto, assume-se que a relação entre o torque gerado e o sinal de controle na saída do conversor D/A é simplesmente dada por um ganho.
} 
com os vetores de estado $x(t)$ e de controle $u(t)$ definidos como:

$$
\begin{aligned}
& x(t)=\left[\begin{array}{lllll}
\psi_{2}(t) & \psi_{1}(t) & \omega_{3}(t) & \omega_{2}(t) & \omega_{1}(t)
\end{array}\right]^{T}, \\
& u(t)=\left[T_{m_{4}}(t) T_{m_{3}}(t)\right]^{T},
\end{aligned}
$$

sendo as matrizes $A, B$ e $C$ :

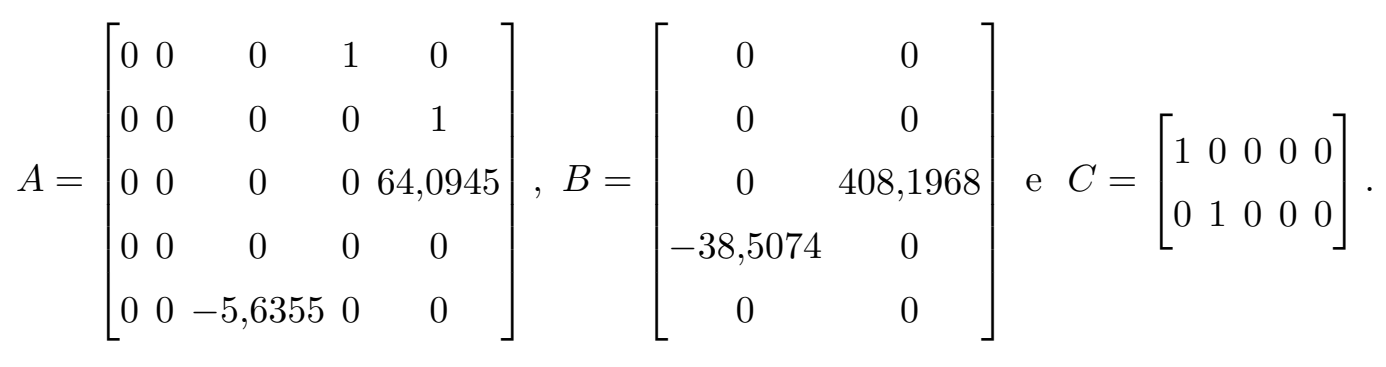

Como esse modelo não possui zeros de transmissão, o sistema é de fase mínima. Ele também é controlável e observável, pois as matrizes de controlabilidade e de observabilidade possuem posto completo. As variáveis a serem controladas são $y_{1}(t)=\psi_{2}(t)$ (posição angular do corpo $B$ - gimbal externo) e $y_{2}(t)=\psi_{1}(t)$ (posição angular do corpo $A$ - base giratória).

As funções de transferência do sistema podem ser encontradas por intermédio da Transformada de Laplace. Assim, das Equações (2.15) e (2.17) chega-se em $\frac{\psi_{2}(s)}{T_{m_{4}}(s)}$. Similarmente, as funções de transferência $\frac{\psi_{1}(s)}{T_{m_{3}}(s)}$ e $\frac{\psi_{3}(s)}{T_{m_{3}}(s)}$ são geradas a partir das Equações (2.16) e (2.18). As expressões resultantes, sem considerar os ganhos dos encoders e dos atuadores, são:

$$
\begin{aligned}
\frac{\psi_{3}(s)}{T_{m_{3}}(s)} & =\frac{0,1331}{0,0036 s^{2}+1,3077} \\
\frac{\psi_{2}(s)}{T_{m_{4}}(s)} & =-\frac{1}{0,0456 s^{2}} \\
\frac{\psi_{1}(s)}{T_{m_{3}}(s)} & =-\frac{1,1435}{0,0036 s^{3}+1,3077 s}
\end{aligned}
$$

Observa-se que o torque aplicado pelo motor \#4 $\left(T_{m_{4}}(t)=u_{1}(t)\right)$ efetua somente o deslocamento angular do gimbal externo $\left(\psi_{2}(t)=y_{1}(t)\right)$ e o torque aplicado pelo motor \#3 $\left(T_{m_{3}}(t)=u_{2}(t)\right)$ efetua, simultaneamente, os deslocamentos angulares do gimbal interno $\left(\psi_{3}(t)\right)$ e da base giratória $\left(\psi_{1}(t)=y_{2}(t)\right)$, caracterizando assim um sistema desacoplado (i.e. a variável de controle $u_{j}(t)$ atua somente na saída $\left.y_{j}(t)(j=1,2)\right)$.

Como nesta dissertação os controladores digitais serão projetados diretamente em tempo discreto, é necessário calcular o equivalente discreto do modelo em tempo contínuo, 
composto por (2.25) a (2.28), por meio dos cálculos descritos na Seção 5.5 em Ogata (1995). Para isso, é necessário definir o valor do período de amostragem $T_{s}$, determinado aqui conforme um critério prático descrito em Castrucci, Bittar e Sales (2011), que diz que uma forma prática de definir $T_{s}$ para o sistema é escolher um valor que seja pelo menos 10 vezes menor que o período das oscilações de saída, se a resposta transitória for subamortecida. Por meio da frequência de nutação obtida em experimentos na Seção F.1.1, chega-se a um período de oscilação de $T_{\text {experimento }} \approx 0,320$ segundos, o que sugere que $T_{s}$ seja menor ou igual à 0,032 segundos. Assim, para esta dissertação, escolhe-se um valor quatro vezes menor que o mínimo sugerido, definindo o período de amostragem como $T_{s}=0,008$ segundos.

Com $T_{s}$ definido, realiza-se os cálculos da discretização utilizando o comando $c 2 d$ do MATLAB ${ }^{\circledR}$ com a opção de discretização por ZOH (zero-order hold), com a seguinte sintaxe: [Phi,Gamma] $=\mathrm{c} 2 \mathrm{~d}(\mathrm{~A}, \mathrm{~B}, \mathrm{C}, 0, \mathrm{Ts})$. Desse modo, obtém-se o seguinte modelo equivalente, na forma de representação em espaço de estados, em tempo discreto:

$$
\left\{\begin{array}{rl}
x[k+1] & =\Phi x[k]+\Gamma u[k] \\
y[k] & =C x[k]
\end{array},\right.
$$

com os vetores de estado $x[k]$ e de controle $u[k]$ equivalentes em tempo discreto:

$$
\begin{aligned}
& x[k]=\left[\psi_{2}[k] \psi_{1}[k] \omega_{3}[k] \omega_{2}[k] \omega_{1}[k]\right]^{T}, \\
& u[k]=\left[T_{m_{4}}[k] T_{m_{3}}[k]\right]^{T},
\end{aligned}
$$

sendo as matrizes $\Phi, \Gamma$ e $C$ :

$$
\Phi=\left[\begin{array}{ccccc}
1 & 0 & 0 & 0,0080 & 0 \\
0 & 1 & -0,0002 & 0 & 0,0080 \\
0 & 0 & 0,9885 & 0 & 0,5108 \\
0 & 0 & 0 & 1 & 0 \\
0 & 0 & -0,0449 & 0 & 0,9885
\end{array}\right], \Gamma=\left[\begin{array}{ccc}
-0,0012 & 0 \\
0 & -0,0002 \\
0 & 3,2530 \\
-0,3081 & 0 \\
0 & -0,0735
\end{array}\right] \text { e } C=\left[\begin{array}{lllll}
1 & 0 & 0 & 0 & 0 \\
0 & 1 & 0 & 0 & 0
\end{array}\right],
$$

as matrizes equivalentes em tempo discreto de $A, B$ e $C$, respectivamente.

Apesar do modelo em tempo discreto resultante continuar sendo controlável e observável, esse método de discretização inseriu três zeros de transmissão, que não existiam no modelo em tempo contínuo, localizados em $z_{\text {trans }}=\{-0,2683 ;-1 ;-3,7271\}$, caracterizando-o como um sistema de fase não-mínima por possuir um zero fora do circulo unitário no plano-z. Como apontado em Astrom, Hagander e Sternby (1980) e Hagiwara, Yuasa e Araki (1992), sistemas com ordem relativa maior que dois podem resultar em 
sistemas discretos com zeros de fase não-mínima.

\subsubsection{Modelo Acoplado da Planta}

As outras configurações possíveis geram modelos lineares multivariáveis acoplados da planta. Os pontos de operação (2.9) dessas configurações são definidos em:

$$
\Omega \neq 0 \mathrm{rad} / \mathrm{s} \text { e } \psi_{2_{0}}=c t e .(\mathrm{em} \mathrm{rad}) \text { e } \psi_{3_{0}} \neq 0 \mathrm{rad}
$$

sendo $0<\Omega \leq 800 \mathrm{RPM}(\approx 83,776 \mathrm{rad} / \mathrm{s}), 0 \leq\left|\psi_{2_{0}}\right|<90^{\circ}(\approx 1,571 \mathrm{rad})$, e $0<\left|\psi_{3_{0}}\right|<$ $90^{\circ}(\approx 1,571 \mathrm{rad})$.

Independentemente dos valores de $\Omega$ e $\psi_{3_{0}}$ escolhidos (dentro dos intervalos definidos acima), para valores de $\psi_{2_{0}}$ negativos ou nulo, geram-se modelos acoplados de fase mínima, e para valores de $\psi_{2_{0}}$ positivos, geram-se modelos acoplados de fase não-mínima.

\subsubsection{Caso Acoplado de Fase Mínima}

O ponto de operação (2.9) de uma das configurações exploradas nesta dissertação é definido em:

$$
\Omega=41,888 \mathrm{rad} / \mathrm{s} ; \quad \psi_{2_{0}}=-20^{\circ} \approx-0,349 \mathrm{rad} ; \quad \text { e } \psi_{3_{0}}=20^{\circ} \approx 0,349 \mathrm{rad}
$$

Ao avaliar as equações linearizadas da planta (2.10) a (2.13) nesse ponto de operação, considerando os valores numéricos dos momentos de inércia dos corpos descritos na Tabela 1 , obtêm-se respectivamente:

$$
\begin{aligned}
T_{m_{4}} & -0,0273 \dot{\omega}_{4}-0,0257 \dot{\omega}_{2}-0,0088 \dot{\omega}_{1}=0, \\
T_{m_{3}} & -0,0272 \dot{\omega}_{3}-0,0093 \dot{\omega}_{1}-0,3911 \omega_{2}+1,0098 \omega_{1}=0, \\
& -0,0257 \dot{\omega}_{4}-0,0704 \dot{\omega}_{2}-0,0065 \dot{\omega}_{1}+0,3911 \omega_{3}+0,1338 \omega_{1}=0, \\
& -0,0088 \dot{\omega}_{4}-0,0093 \dot{\omega}_{3}-0,0065 \dot{\omega}_{2}-0,1325 \dot{\omega}_{1}-1,0098 \omega_{3}-0,1338 \omega_{2}=0,
\end{aligned}
$$

chegando-se às seguintes equações:

$$
\begin{aligned}
& \dot{\omega}_{3}=2,6918 \omega_{3}-14,3792 \omega_{2}+38,0446 \omega_{1}+0,9657 T_{m_{4}}+37,69 T_{m_{3}}, \\
& \dot{\omega}_{2}=8,1534 \omega_{3}+2,7886 \omega_{1}-20,4121 T_{m_{4}}-0,1024 T_{m_{3}}, \\
& \dot{\omega}_{1}=-7,8702 \omega_{3}-2,6918 \omega_{1}-2,8236 T_{m_{4}}-2,7054 T_{m_{3}} .
\end{aligned}
$$

De forma semelhante à realizada no caso desacoplado, os ganhos dos encoders e dos 
atuadores devem ser considerados em (2.42) a (2.44), resultando em:

$$
\begin{aligned}
\dot{\omega}_{3} & =\left(2,6918 \frac{\omega_{3}}{k_{e_{3}}}-14,3792 \frac{\omega_{2}}{k_{e_{2}}}+38,0446 \frac{\omega_{1}}{k_{e_{1}}}+0,9657 T_{m_{4}} k_{m_{4}}+37,69 T_{m_{3}} k_{m_{3}}\right) k_{e_{3}}, \\
\dot{\omega}_{2} & =\left(8,1534 \frac{\omega_{3}}{k_{e_{3}}}+2,7886 \frac{\omega_{1}}{k_{e_{1}}}-20,4121 T_{m_{4}} k_{m_{4}}-0,1024 T_{m_{3}} k_{m_{3}}\right) k_{e_{2}}, \\
\dot{\omega}_{1} & =\left(-7,8702 \frac{\omega_{3}}{k_{e_{3}}}-2,6918 \frac{\omega_{1}}{k_{e_{1}}}-2,8236 T_{m_{4}} k_{m_{4}}-2,7054 T_{m_{3}} k_{m_{3}}\right) k_{e_{1}},
\end{aligned}
$$

e ao substituir esses ganhos pelos valores da Tabela 1, tem-se que:

$$
\begin{aligned}
& \dot{\omega}_{3}=2,6918 \omega_{3}-21,9216 \omega_{2}+58,0004 \omega_{1}+2,5852 T_{m_{4}}+418,4703 T_{m_{3}}, \\
& \dot{\omega}_{2}=5,3481 \omega_{3}+2,7886 \omega_{1}-35,8423 T_{m_{4}}-0,7458 T_{m_{3}}, \\
& \dot{\omega}_{1}=-5,1624 \omega_{3}-2,6918 \omega_{1}-4,9580 T_{m_{4}}-19,7029 T_{m_{3}} .
\end{aligned}
$$

As matrizes $A, B$ e $C$ resultantes das Equações (2.48) a (2.50) escritas na forma de representação em espaço de estados em tempo contínuo (2.25) a (2.27) são:

$$
A=\left[\begin{array}{ccccc}
0 & 0 & 0 & 1 & 0 \\
0 & 0 & 0 & 0 & 1 \\
0 & 0 & 2,6918 & -21,9216 & 58,0004 \\
0 & 0 & 5,3481 & 0 & 2,7886 \\
0 & 0 & -5,1624 & 0 & -2,6918
\end{array}\right], B=\left[\begin{array}{ccc}
0 & 0 \\
0 & 0 \\
2,5852 & 418,4703 \\
-35,8423 & -0,7458 \\
-4,9580 & -19,7029
\end{array}\right] \text { e } C=\left[\begin{array}{lllll}
1 & 0 & 0 & 0 & 0 \\
0 & 1 & 0 & 0 & 0
\end{array}\right]
$$

Este modelo em tempo contínuo apresenta um zero de transmissão localizado em $s_{\text {trans }}=-122,9223$. Como esse zero está localizado no semi-plano esquerdo do plano-s, o sistema é de fase mínima. Ele também é controlável e observável.

As seguintes matrizes $\Phi, \Gamma$ e $C$ do modelo em espaço de estados em tempo discreto com ZOH são dadas por:

$$
\Phi=\left[\begin{array}{ccccc}
1 & 0 & 0,0002 & 0,0080 & 0,0001 \\
0 & 1 & -0,0002 & 0 & 0,0079 \\
0 & 0 & 1,0084 & -0,1765 & 0,4600 \\
0 & 0 & 0,0426 & 0,9963 & 0,0319 \\
0 & 0 & -0,0411 & 0,0036 & 0,9692
\end{array}\right], \Gamma=\left[\begin{array}{ccc}
-0,0011 & 0,0002 \\
-0,0002 & -0,0008 \\
0,0369 & 3,3333 \\
-0,2865 & 0,0632 \\
-0,0399 & -0,2244
\end{array}\right] \text { e } C=\left[\begin{array}{lllll}
1 & 0 & 0 & 0 & 0 \\
0 & 1 & 0 & 0 & 0
\end{array}\right] .
$$

Os zeros de transmissão estão localizados em $z_{\text {trans }}=\{0,3723 ;-1 ;-1,3591\}$ no plano$z$, o que também o caracteriza como um sistema de fase não-mínima. 


\subsubsection{Caso Acoplado de Fase Não-Mínima}

O ponto de operação da outra configuração explorada nesta dissertação é definido em:

$$
\Omega=41,888 \mathrm{rad} / \mathrm{s} ; \quad \psi_{2_{0}}=20^{\circ} \approx 0,349 \mathrm{rad} ; \text { e } \psi_{3_{0}}=-20^{\circ} \approx-0,349 \mathrm{rad}
$$

Ao avaliar as equações linearizadas da planta (2.10) a (2.13) nesse ponto de operação, considerando os valores numéricos dos momentos de inércia dos corpos descritos na Tabela 1, obtêm-se respectivamente:

$$
\begin{aligned}
T_{m_{4}}- & 0,0273 \dot{\omega}_{4}-0,0257 \dot{\omega}_{2}+0,0088 \dot{\omega}_{1}=0, \\
T_{m_{3}}- & 0,0272 \dot{\omega}_{3}+0,0093 \dot{\omega}_{1}+0,3911 \omega_{2}+1,0098 \omega_{1}=0, \\
- & 0,0257 \dot{\omega}_{4}-0,0704 \dot{\omega}_{2}+0,0065 \dot{\omega}_{1}-0,3911 \omega_{3}+0,1338 \omega_{1}=0, \\
& 0,0088 \dot{\omega}_{4}+0,0093 \dot{\omega}_{3}+0,0065 \dot{\omega}_{2}-0,1325 \dot{\omega}_{1}-1,0098 \omega_{3}-0,1338 \omega_{2}=0,
\end{aligned}
$$

chegando-se às seguintes equações:

$$
\begin{aligned}
& \dot{\omega}_{3}=-2,6918 \omega_{3}+14,3792 \omega_{2}+38,0446 \omega_{1}+0,9657 T_{m_{4}}+37,69 T_{m_{3}}, \\
& \dot{\omega}_{2}=-8,1534 \omega_{3}+2,7886 \omega_{1}-20,4121 T_{m_{4}}-0,1024 T_{m_{3}}, \\
& \dot{\omega}_{1}=-7,8702 \omega_{3}+2,6918 \omega_{1}+2,8236 T_{m_{4}}+2,7054 T_{m_{3}} .
\end{aligned}
$$

Ao considerar os ganhos dos encoders e dos atuadores em (2.58) a (2.60), tem-se que:

$$
\begin{aligned}
\dot{\omega}_{3} & =\left(-2,6918 \frac{\omega_{3}}{k_{e_{3}}}+14,3792 \frac{\omega_{2}}{k_{e_{2}}}+38,0446 \frac{\omega_{1}}{k_{e_{1}}}+0,9657 T_{m_{4}} k_{m_{4}}+37,69 T_{m_{3}} k_{m_{3}}\right) k_{e_{3}}, \\
\dot{\omega}_{2} & =\left(-8,1534 \frac{\omega_{3}}{k_{e_{3}}}+2,7886 \frac{\omega_{1}}{k_{e_{1}}}-20,4121 T_{m_{4}} k_{m_{4}}-0,1024 T_{m_{3}} k_{m_{3}}\right) k_{e_{2}}, \\
\dot{\omega}_{1} & =\left(-7,8702 \frac{\omega_{3}}{k_{e_{3}}}+2,6918 \frac{\omega_{1}}{k_{e_{1}}}+2,8236 T_{m_{4}} k_{m_{4}}+2,7054 T_{m_{3}} k_{m_{3}}\right) k_{e_{1}},
\end{aligned}
$$

e ao substituir esses ganhos pelos valores da Tabela 1, tem-se que:

$$
\begin{aligned}
& \dot{\omega}_{3}=-2,6918 \omega_{3}+21,9216 \omega_{2}+58,0004 \omega_{1}+2,5852 T_{m_{4}}+418,4703 T_{m_{3}}, \\
& \dot{\omega}_{2}=-5,3481 \omega_{3}+2,7886 \omega_{1}-35,8423 T_{m_{4}}-0,7458 T_{m_{3}} \\
& \dot{\omega}_{1}=-5,1624 \omega_{3}+2,6918 \omega_{1}+4,9580 T_{m_{4}}+19,7029 T_{m_{3}} .
\end{aligned}
$$

As matrizes $A, B$ e $C$ resultantes das Equações (2.64) a (2.66) escritas na forma de 
representação em espaço de estados em tempo contínuo (2.25) a (2.27) são:

$$
A=\left[\begin{array}{ccccc}
0 & 0 & 0 & 1 & 0 \\
0 & 0 & 0 & 0 & 1 \\
0 & 0 & -2,6918 & 21,9216 & 58,0004 \\
0 & 0 & -5,3481 & 0 & 2,7886 \\
0 & 0 & -5,1624 & 0 & 2,6918
\end{array}\right], B=\left[\begin{array}{ccc}
0 & 0 \\
0 & 0 \\
2,5852 & 418,4703 \\
-35,8423 & -0,7458 \\
4,9580 & 19,7029
\end{array}\right] \text { e } C=\left[\begin{array}{lllll}
1 & 0 & 0 & 0 & 0 \\
0 & 1 & 0 & 0 & 0
\end{array}\right] \text {. }
$$

Este modelo em tempo contínuo apresenta um zero de transmissão localizado em $s_{\text {trans }}=122,9223$. Como esse zero está localizado no semi-plano direito do plano-s, o sistema é de fase não-mínima, embora seja controlável e observável.

As matrizes do modelo em espaço de estados do equivalente em tempo discreto com ZOH são dadas por:

$$
\Phi=\left[\begin{array}{ccccc}
1 & 0 & -0,0002 & 0,0080 & 0,0001 \\
0 & 1 & -0,0002 & 0 & 0,0081 \\
0 & 0 & 0,9655 & 0,1727 & 0,4639 \\
0 & 0 & -0,0426 & 0,9963 & 0,0125 \\
0 & 0 & -0,0411 & -0,0036 & 1,0121
\end{array}\right], \Gamma=\left[\begin{array}{ccc}
-0,0011 & -0,0002 \\
0,0002 & 0,0004 \\
0,0047 & 3,3333 \\
-0,2865 & -0,0762 \\
0,0399 & 0,0899
\end{array}\right] \text { e } C=\left[\begin{array}{lllll}
1 & 0 & 0 & 0 & 0 \\
0 & 1 & 0 & 0 & 0
\end{array}\right]
$$

Este modelo possui três zeros de transmissão localizados em $z_{\text {trans }}=\{-0,7358 ;-1 ; 2,6863\}$ no plano- $z$, mantendo a característica de um sistema de fase não-mínima. 


\section{ESTRATÉGIAS DE CONTROLE}

Nesta dissertação são exploradas técnicas de controle digital para a execução de projetos de controladores digitais, com o objetivo de controlar a atitude da planta giroscópio, ou seja, fazer com que as saídas do sistema $y[k]=\left[\psi_{2}[k] \psi_{1}[k]\right]^{T}$ acompanhem sinais de referência $r[k]=\left[r_{\psi_{2}}[k] r_{\psi_{1}}[k]\right]^{T}$, como também rejeitem sinais de perturbação $d[k]=\left[d_{\theta_{2}}[k] d_{\theta_{1}}[k]\right]^{T}$, conforme o diagrama de blocos da Figura 4.

Figura 4: Diagrama de blocos considerado para o projeto dos controladores

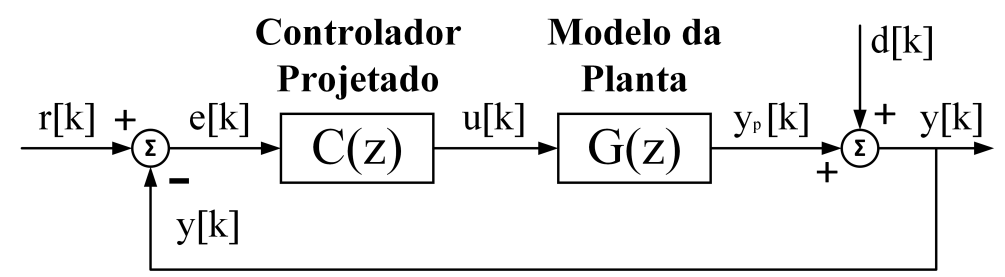

Fonte: Autor.

As velocidades angulares dos corpos $\omega_{n}=\dot{\psi}_{n}$ são indiretamente obtidas aqui pelo método retangular para trás (backward retangular rule) (FRANKLIN; POWELL; WORKMAN, 1997), a partir das medidas dos encoders incrementais.

\subsection{Controle PID Digital}

Para a estratégia de controle PID digital, são considerados nesta dissertação somente os elementos da diagonal principal da matriz de transferência em tempo discreto $G(z)$ da planta e, portanto, define-se o controlador a ser projetado $C(z)$ como uma matriz diagonal:

$$
G(z)=\left[\begin{array}{cc}
G_{1}(z) & 0 \\
0 & G_{2}(z)
\end{array}\right] \text { e } C(z)=\left[\begin{array}{cc}
C_{1}(z) & 0 \\
0 & C_{2}(z)
\end{array}\right]
$$

resultando na seguinte matriz de transferência de malha aberta do sistema:

$$
G(z) C(z)=\left[\begin{array}{cc}
G_{1}(z) C_{1}(z) & 0 \\
0 & G_{2}(z) C_{2}(z)
\end{array}\right] .
$$

Portanto, os elementos $C_{q}(z)$ da diagonal principal do controlador são projetados em 
relação aos elementos $G_{q}(z)^{1}(q=1,2)$. Para isso, é considerada a função de transferência discreta do controlador PID digital básico, com aproximações pelo método de Tustin para a parte integral e pelo método retangular para trás para a parte derivativa, dada por: (CASTRUCCI; BITTAR; SALES, 2011)

$$
\begin{aligned}
C_{q}(z) & =K_{p_{q}}\left(1+\frac{T_{s}}{2 T_{i_{q}}} \frac{(z+1)}{(z-1)}+\frac{T_{d_{q}}}{T_{s}} \frac{(z-1)}{z}\right)=a_{q}+b_{q} \frac{(z+1)}{(z-1)}+c_{q} \frac{(z-1)}{z}= \\
& =\frac{a_{q} z(z-1)+b_{q} z(z+1)+c_{q}(z-1)^{2}}{z(z-1)}
\end{aligned}
$$

$\operatorname{com} a_{q}=K_{p_{q}}, b_{q}=K_{p_{q}} \frac{T_{s}}{2 T_{i_{q}}}$ e $c_{q}=K_{p_{q}} \frac{T_{d_{q}}}{T_{s}}$.

De forma equivalente, pode-se escrever (3.3) como:

$$
C_{q}(z)=\frac{K_{q}\left(z-\alpha_{q}\right)\left(z-\beta_{q}\right)}{z(z-1)} .
$$

Assim, pode-se aplicar o método do lugar das raízes (L.R.) para o projeto do controlador, no qual $\alpha_{q}$ geralmente é escolhido de forma a cancelar um polo estável do elemento $G_{q}(z)^{2}$. Por conseguinte, como $G_{q}(z) C_{q}(z)$ é uma grandeza complexa, $\beta_{q}$ é definido de forma a atender a condição angular:

$$
\angle G_{q}(z) C_{q}(z)= \pm \pi(2 k+1), \text { sendo } k=0,1,2, \ldots
$$

e $K_{q}$ é definido de forma a atender a condição de módulo:

$$
\left|G_{q}(z) C_{q}(z)\right|=1
$$

sendo os valores de $z$, que atendem essas condições, os polos de malha fechada do sistema.

Portanto, pode-se alocar um par de polos complexos conjugados desejados de malha fechada $z_{0_{q}}$ e $z_{0_{q}}^{*}$, definidos, nesta dissertação, por meio das seguintes especificações de desempenho: tempo de acomodação $t_{s_{q}}(5 \%)^{3}$, em segundos, e máximo sobressinal percentual da resposta ao degrau $M_{p_{q}}(\%)^{4}$, em porcentagem. Com essas duas especificações

\footnotetext{
${ }^{1}$ Para facilitar o projeto de $C_{q}(z)$, os elementos $G_{q}(z)$ que forem negativos terão o seu sinal invertido no início do projeto, tornando-o positivo somente para o projeto do controlador, o que causará uma inversão de sinal em $K_{q}$ de $C_{q}(z)$, que será considerado no projeto do controlador PID digital na Seção 4.1.

${ }^{2}$ porém, no caso desta planta giroscópio, $G_{q}(z)$ não possui nenhum polo estável. Então, escolhe-se $\alpha_{q}$ com um valor real, dentro do circulo unitário $\left(0<\left|\alpha_{q}\right|<1\right)$.

${ }^{3}$ tempo em que a resposta alcança e permanece dentro de uma faixa de erro de $\pm 5 \%$ do valor de regime estacionário.

${ }^{4}$ dada por $M_{p}(\%)=\frac{\Delta_{p}-\Delta_{f}}{\Delta_{f}} \cdot 100 \%$, sendo $\Delta_{p}=v_{p}-v_{i}$ e $\Delta_{f}=v_{\infty}-v_{i}$, nos quais $v_{p}, v_{i}$ e $v_{\infty}$ são os valores de pico, inicial e de regime estacionário da resposta, respectivamente.
} 
são calculados o coeficiente de amortecimento $\zeta_{q}$ e a frequência natural $\omega_{n_{q}}$, que definem a localização desse par de polos no plano- $z$, dada por: (OGATA, 1995)

$$
z_{0_{q}}=e^{T_{S}\left(-\zeta_{q} \omega_{n_{q}} \pm j \omega_{n_{q}} \sqrt{1-\zeta_{q}^{2}}\right)}
$$

com período de amostragem $T_{s}=0,008$ segundos.

Após calculado $C_{q}(z)$, podem-se calcular as componentes $K_{p_{q}}, T_{i_{q}}$ e $T_{d_{q}}$ de (3.3) pelas seguintes equações:

$$
\begin{aligned}
K_{p_{q}} & =\frac{K_{q} \Upsilon_{q}}{2} ; \\
T_{i_{q}} & =\frac{T_{s} \Upsilon_{q}}{2\left(1+\alpha_{q} \beta_{q}-\alpha_{q}-\beta_{q}\right)} \\
T_{d_{q}} & =\frac{2 T_{s} \alpha_{q} \beta_{q}}{\Upsilon_{q}} .
\end{aligned}
$$

sendo $\Upsilon_{q}=\alpha_{q}+\beta_{q}-3 \alpha_{q} \beta_{q}+1$.

\subsection{Controle LQR Digital com Ação Integrativa}

Como objetiva-se o controle de atitude da planta para a estratégia de controle LQR digital, é considerada, primeiramente, uma inserção de integradores na malha de controle, como apresenta a Figura 5, onde são considerados os modelos obtidos em tempo discreto da planta na Seção 2.6, com uma realimentação de estados. Essa inserção gera um modelo aumentado da planta, no qual a estratégia de controle LQR digital é baseada e explorada.

Figura 5: Diagrama de blocos do sistema com controle LQR e integradores

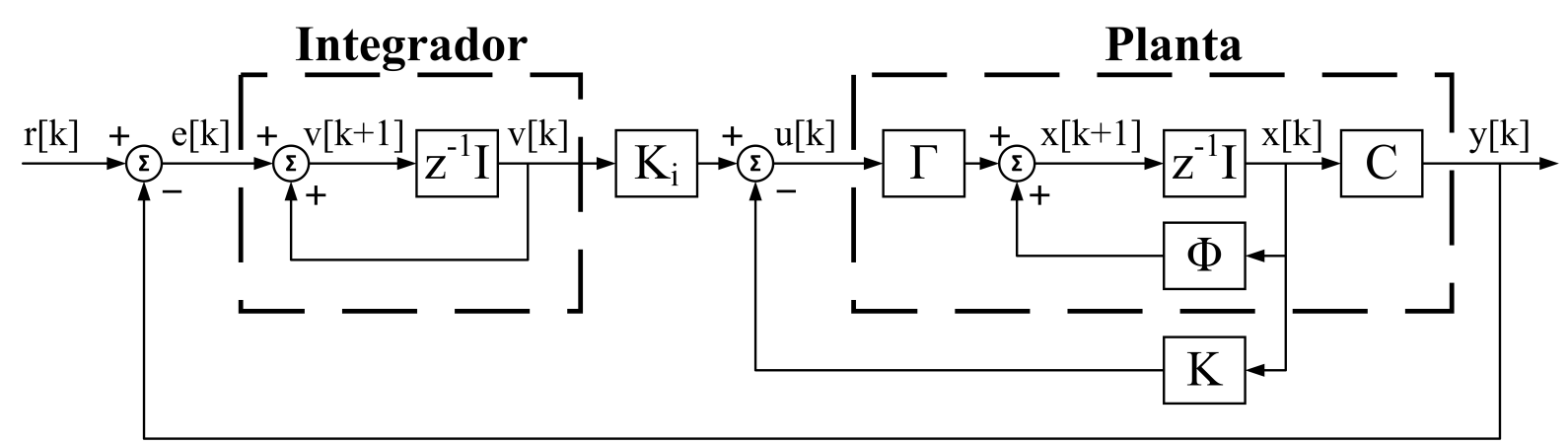

Fonte: Autor. 


\subsubsection{Inserção dos Integradores}

O sistema da Figura 5 é composto pelos seguintes elementos:

- $u[k]$ : vetor de controle, com dimensão $n_{u}$;

- $y[k]$ : vetor de saída, com dimensão $n_{y}$;

- $x[k]$ : vetor de estados da planta, com dimensão $n_{x}$;

- $v[k]$ : vetor de estados dos integradores inseridos, com dimensão $n_{v}=n_{y}$;

- $r[k]$ : vetor de referência, com dimensão $n_{r}=n_{y}$;

- $e[k]$ : vetor de erro de acompanhamento de referência, com dimensão $n_{e}=n_{y}$;

- $\Phi$ : matriz de estados da planta, com dimensão $n_{x} \times n_{x}$;

- $\Gamma$ : matriz de controle da planta, com dimensão $n_{x} \times n_{u}$;

- $C$ : matriz de saída da planta, com dimensão $n_{y} \times n_{x}$;

- $K$ : matriz de realimentação de estados da planta, com dimensão $n_{u} \times n_{x}$; e

- $K_{i}$ : matriz de realimentação de estados dos integradores, com dimensão $n_{u} \times n_{v}$.

A inserção dos integradores na malha de controle é realizada para corrigir o desvio da saída $y[k]$ em relação ao sinal de referência $r[k]$, na qual os integradores discretos obtêm a integral dos sinais de erros de acompanhamento de referência $e[k]=r[k]-y[k]$, e seu vetor de estados $v[k]$ altera enquanto $e[k]$ não for nula. Apesar da planta possuir polos em $z=1$ no plano- $z^{5}$, integradores são necessários devido aos efeitos do atrito nas juntas da planta.

Pela Figura 5, observa-se que a equação de estados dos integradores inseridos é dada por:

$$
v[k+1]=v[k]+r[k]-\underbrace{C x[k]}_{y[k]},
$$

o vetor de controle por:

$$
u[k]=K_{i} v[k]-K x[k],
$$

\footnotetext{
${ }^{5}$ equivalente a possuir polos na origem do plano- $s$, no caso em tempo contínuo.
} 
e, com a realimentação de estados na planta, a equação de estados do sistema em malha fechada é dada agora por:

$$
x[k+1]=(\Phi-\Gamma K) x[k]+\Gamma K_{i} v[k] .
$$

Para projetar as matrizes de ganho de realimentação de estados $K_{i}$ (para os estados dos integradores $v[k]$ ) e $K$ (para os estados da planta $x[k]$ ), o sistema aumentado em malha fechada, em tempo discreto, é considerado e composto por (3.11) e (3.13):

$$
\underbrace{\left[\begin{array}{l}
x[k+1] \\
v[k+1]
\end{array}\right]}_{x_{\text {aum }}[k+1]}=\underbrace{\left[\begin{array}{cc}
\Phi-\Gamma K & \Gamma K_{i} \\
-C & I_{n_{v} \times n_{v}}
\end{array}\right]}_{A m p} \cdot \underbrace{\left[\begin{array}{l}
x[k] \\
v[k]
\end{array}\right]}_{x_{\text {aum }}[k]}+\left[\begin{array}{c}
0_{n_{x} \times n_{r}} \\
I_{n_{v} \times n_{r}}
\end{array}\right] \cdot r[k],
$$

com a matriz Amp reescrita como:

$$
A m p=\underbrace{\left[\begin{array}{cc}
\Phi & 0_{n_{x} \times n_{v}} \\
-C & I_{n_{v} \times n_{v}}
\end{array}\right]}_{\Phi_{\text {aum }}}-\underbrace{\left[\begin{array}{c}
\Gamma \\
0_{n_{v} \times n_{u}}
\end{array}\right]}_{\Gamma_{\text {aum }}} \cdot \underbrace{\left[K-K_{i}\right]}_{K_{\text {aum }}},
$$

sendo $I$ e 0 as matrizes identidade e nula, respectivamente, com suas devidas dimensões; $x_{\text {aum }}[k]$ o vetor de estados do sistema aumentado, com dimensão $\left(n_{x}+n_{v}\right) ; \Phi_{\text {aum }}$ a matriz de estados do sistema aumentado, com dimensão $\left(n_{x}+n_{v}\right) \times\left(n_{x}+n_{v}\right) ; \Gamma_{\text {aum }}$ a matriz de controle do sistema aumentado, com dimensão $\left(n_{x}+n_{v}\right) \times n_{u}$; e $K_{\text {aum }}$ a matriz de realimentação do sistema aumentado, com dimensão $n_{u} \times\left(n_{x}+n_{v}\right)$.

Ao comparar (3.14) e (3.15) com (3.13), observa-se que o ganho $K_{\text {aum }}$ pode ser encontrado considerando o sistema aumentado $\left(\Phi_{\text {aum }}, \Gamma_{\text {aum }}\right)$, como $K$ pode ser encontrado para o sistema original $(\Phi, \Gamma)$. O ganho $K_{i}$ é dado pelas $n_{v}$ últimas colunas de $K_{\text {aum }}$ com sinal trocado. O sistema aumentado $\left(\Phi_{\text {aum }}, \Gamma_{\text {aum }}\right)$ é controlável se, e somente se, o sistema original $(\Phi, \Gamma)$ é controlável (FADALI M. SAMI; VISIOLI, 2013).

\subsubsection{Estratégia de Controle LQR Digital}

Primeiramente é apresentado, de forma breve, a teoria de Controle Ótimo Quadrático considerando o sistema aumentado $\left(\Phi_{\text {aum }}, \Gamma_{\text {aum }}\right)$ e, em seguida, seu caso particular ao considerar o problema em regime estacionário - o controle LQR. Nele são utilizadas a regra de Bryson, para a escolha das matrizes $Q$ e $R$, e o procedimento de pincer, para tentar impor uma especificação de desempenho ao problema - o tempo de acomodação $t_{s}$ dos estados $x_{\text {aum }}[k]$. 


\subsubsection{Controle Ótimo Quadrático (COQ)}

Como demonstrado no Capitulo 8 em Ogata (1995), o Controle Ótimo "otimiza" (minimiza ou maximiza) uma determinada função escolhida como índice de desempenho (performance index). A função escolhida busca levar os estados para zero e, ao mesmo tempo, minimizar o esforço de controle. Por outro lado, ao considerar o sistema aumentado $\left(\Phi_{\text {aum }}, \Gamma_{\text {aum }}\right)$, a função escolhida busca levar os estados para um valor constante $x_{\text {aum }}$ (não necessariamente igual a zero), que depende do sinal de referência $r[N]$.

Para a estratégia de Controle Ótimo Quadrático digital, considera-se um processo de controle finito ${ }^{6}$ para problema de regulação ${ }^{7}$ do sistema aumentado $\left(\Phi_{\text {aum }}, \Gamma_{\text {aum }}\right)$, com as condições iniciais $x_{\text {aum }}[0]$ conhecidas, e determina-se um vetor de controle $u[k]$, tal que o seguinte índice de desempenho seja minimizado:

$$
J_{(C O Q)}=\frac{1}{2} x_{\text {aum }}^{T}[N] S x_{\text {aum }}[N]+\frac{1}{2} \sum_{k=0}^{N-1}\left(x_{\text {aum }}^{T}[k] Q x_{\text {aum }}[k]+u^{T}[k] R u[k]\right),
$$

sendo $S$ e $Q$ matrizes simétricas de dimensões $\left(n_{x}+n_{v}\right) \times\left(n_{x}+n_{v}\right)$, definida ou semidefinida positivas, de ponderação do índice de desempenho, relativas ao vetor de estados e ao vetor de estados final, respectivamente, e $R$ matriz simétrica de dimensão $n_{u} \times n_{u}$, definida positiva, de ponderação do índice de desempenho, relativa ao vetor de controle. O primeiro termo da função custo $J_{(C O Q)}$ leva em conta o peso do vetor de estados no instante final de amostragem $N$, o primeiro termo dentro do somatório leva em conta o peso do erro durante o processo de controle, e o segundo leva em conta o peso do sinal de controle $u[k]$.

Há várias formas de calcular a solução do problema de minimização de $J_{(C O Q)}$, uma delas é pelo método de multiplicadores de Lagrange (Lagrange Multipliers), demonstrada na Seção 8.2 em Ogata (1995), no qual obtém-se o vetor de controle "ótimo" que minimiza (3.16), considerando que o sistema seja controlável. Sua forma equivalente para o sistema aumentado, em tempo discreto, é dada por:

$$
u[k]=-K_{\text {aum }}[k] x_{\text {aum }}[k],
$$

sendo $K_{\text {aum }}[k]$ a matriz de ganhos de realimentação variante no tempo, de dimensão

\footnotetext{
${ }^{6}$ ou seja, o instante final de amostragem $N$ é finito $(0 \leq k \leq N)$.

${ }^{7}$ é considerado o sistema em modo regulatório, ou seja, pretende-se que $x_{a u m}[N]$ convirja a um valor $x_{a_{u m}}$ no instante final de amostragem $N$, que é imposto por $r[N]$.
} 
$n_{u} \times\left(n_{x}+n_{v}\right)$, dada por:

$$
K_{\text {aum }}[k]=\left(R+\Gamma_{\text {aum }}^{T} P[k+1] \Gamma_{\text {aum }}\right)^{-1} \Gamma_{\text {aum }}^{T} P[k+1] \Phi_{\text {aum }},
$$

sendo a matriz $P[k]$, de dimensão $\left(n_{x}+n_{v}\right) \times\left(n_{x}+n_{v}\right)$, obtida pela equação de Riccati:

$$
P[k]=Q+\Phi_{\text {aum }}^{T} P[k+1]\left[I_{\left(n_{x}+n_{v}\right) \times\left(n_{x}+n_{v}\right)}-\Gamma_{\text {aum }}\left(R+\Gamma_{\text {aum }}^{T} P[k+1] \Gamma_{\text {aum }}\right)^{-1} \Gamma_{\text {aum }}^{T} P[k+1]\right] \Phi_{\text {aum }},
$$

assumindo que exista a inversa de $\left(R+\Gamma_{\text {aum }}^{T} P[k+1] \Gamma_{\text {aum }}\right)$.

No instante final de amostragem $k=N$, tem-se que $P[N]=S$. Com isso, dados $Q, R$ e $S$, pode-se resolver (3.19) de forma recursiva a partir de $k=N-1$ até $k=0$ e, então, calcular (3.18).

\subsubsection{Regulador Linear Quadrático (LQR)}

O Regulador Linear Quadrático (LQR - Linear Quadratic Regulator) é um tipo particular do Controle Ótimo Quadrático, no qual considera-se um processo de controle sem fim, ou seja, conforme $N$ se aproxima do infinito, a solução do controle ótimo se torna uma solução de regime estacionário, e a matriz de ganho de realimentação $K_{\text {aum }}[k]$ se torna uma matriz de ganho $K_{\text {aum }}$ constante (OGATA, 1995). Esta matriz $K_{\text {aum }}$, também chamada de matriz de ganho de regime estacionário, é mais fácil de ser implementada em um sistema de controle, no qual a solução com este ganho é a "ótima" para este caso, sendo não "ótima" somente durante o regime transitório. (FRANKLIN; POWELL; WORKMAN, 1997)

Para o caso do controle LQR, considera-se que $N$ se aproxima do infinito e, portanto, pode-se alterar o índice de desempenho para:

$$
J_{(L Q R)}=\frac{1}{2} \sum_{k=0}^{\infty}\left[x_{a u m}^{T}[k] Q x_{a u m}[k]+u^{T}[k] R u[k]\right],
$$

pois, se o regulador ótimo é estável, então $J_{(L Q R)}$ converge para um valor constante, sendo que $x_{\text {aum }}[\infty]$ converge a um valor $x_{a u m_{f}}$ constante de regime estacionário, que é imposto por $r[\infty]$ e, consequentemente, o primeiro termo de $J_{(C O Q)}$ pode ser removido no índice de desempenho $J_{(L Q R)}$ do regulador ótimo. (OGATA, 1995)

Portanto, resta a escolha das matrizes $Q$ e $R$, que é realizada de forma empírica conforme os requisitos desejados do projeto a ser realizado. As matrizes $Q$ e $R$ definem o peso relativo que o vetor de estados e os sinais de controle, respectivamente, terão no critério quadrático a ser minimizado. Como o primeiro termo dentro do somatório de 
$J_{(L Q R)}$ é maior ou igual a zero pata todo $k \geq 0$, este termo, influenciado pela matriz $Q$, penaliza desvios dos estados. E como o segundo termo é maior que zero para todo $k \geq 0$ e $u[k] \neq 0$, este termo, influenciado pela matriz $R$, penaliza as trajetórias do sinal de controle. Portanto, deve ser estabelecido um compromisso entre essas duas quantidades por meio da escolha de $Q$ e $R$, lembrando que $Q$ é uma matriz definida ou semi-definida positiva e $R$ é uma matriz definida positiva.

Uma das formas de escolher as matrizes $Q$ e $R$ é pela regra de Bryson, mencionada na Seção 7.6 em Franklin, Powell e Emami-Naeini (2009), que sugere um método razoável para obter valores aceitáveis de $x_{a u m}[k]$ e $u[k]$, em que são escolhidas inicialmente matrizes diagonais $Q$ e $R$ tais que:

$$
\begin{aligned}
Q_{i i} & =\frac{1}{\text { Máxima variação aceitável para } x_{a u m_{i}}^{2}}, \quad i=1,2,3, \ldots,\left(n_{x}+n_{v}\right) ; \\
R_{j j} & =\frac{1}{\text { Máxima variação aceitável para } u_{j}^{2}}, \quad j=1, \ldots, n_{u} .
\end{aligned}
$$

Essas matrizes são, então, ajustadas empiricamente ${ }^{8}$ até que seja atingido um compromisso aceitável entre desempenho e esforço de controle do sistema, levando em consideração os requisitos desejados de projeto.

Após definidas as matrizes $Q$ e $R$, por serem fracamente conectadas com as especificações de desempenho, pode-se utilizar o procedimento de pincer, mencionada no Capitulo 9.5 em Franklin, Powell e Workman (1997), para introduzir outro grau de liberdade ao problema, impondo que uma especificação de desempenho, o tempo de acomodação $t_{s}$ dos estados $x_{\text {aum }}$, seja satisfeita. Isso é feito ao impor que todos os polos de malha fechada permaneçam dentro do circulo de raio $\frac{1}{\alpha}$, sendo $\alpha>1$, fazendo com que a resposta transitória de malha fechada decaia com uma taxa de pelo menos $\frac{1}{\alpha^{k}}$. Este efeito é introduzido ao sistema modificando (3.20) para:

$$
J_{p p}=\sum_{k=0}^{\infty}\left(x_{\text {aum }}^{T}[k] Q x_{\text {aum }}[k]+u^{T}[k] R u[k]\right) \alpha^{2 k},
$$

sendo que o termo escalar $\alpha^{2 k}$ pode ser distribuído em (3.23) como $\alpha^{k} \alpha^{k}$, e definindo $v_{1}[k]=\alpha^{k} x_{a u m}[k]$ e $v_{2}[k]=\alpha^{k} u[k]$ resulta em:

$$
J_{p p}=\sum_{k=0}^{\infty}\left(v_{1}^{T}[k] Q v_{1}[k]+v_{2}^{T}[k] R v_{2}[k]\right) .
$$

\footnotetext{
${ }^{8}$ No caso desta dissertação, por meio de simulações com o sistema.
} 
Essa transformação altera o problema no controle LQR, definido pela nova equação de diferenças:

$$
v_{1}[k+1]=\alpha \Phi_{a u m} v_{1}[k]+\alpha \Gamma_{a u m} v_{2}[k]
$$

que possui um vetor de controle $v_{2}[k]$ "ótimo" que minimiza (3.24), considerando que o sistema seja controlável:

$$
\begin{aligned}
v_{2}[k] & =-K_{\text {aum }} v_{1}[k] \Rightarrow \\
\alpha^{k} u[k] & =-K_{\text {aum }}\left(\alpha^{k} x_{\text {aum }}[k]\right),
\end{aligned}
$$

$\mathrm{Ou}$

$$
u[k]=-K_{\text {aum }} x_{\text {aum }}[k]
$$

que possui a mesma forma do vetor de controle "ótimo" que minimiza a função custo (3.20) do problema original no controle LQR. Então, a matriz de ganhos de realimentação constante $K_{\text {aum }}$ é dada agora por:

$$
K_{\text {aum }}=\left(R+\alpha^{2} \Gamma_{\text {aum }}^{T} P_{\text {ss }} \Gamma_{\text {aum }}\right)^{-1} \alpha^{2} \Gamma_{\text {aum }}^{T} P_{\text {ss }} \Phi_{\text {aum }}
$$

sendo a matriz $P_{s s}$, de dimensão $\left(n_{x}+n_{v}\right) \times\left(n_{x}+n_{v}\right)$, obtida pela solução da equação de Riccati em sua forma estacionária ${ }^{9}$ :

$$
Q-P_{s s}+\alpha^{2} \Phi_{a u m}^{T} P_{s s}\left[I_{\left(n_{x}+n_{v}\right) \times\left(n_{x}+n_{v}\right)}-\alpha^{2} \Gamma_{a u m}\left(R+\alpha^{2} \Gamma_{a u m}^{T} P_{s s} \Gamma_{a u m}\right)^{-1} \Gamma_{a u m}^{T} P_{s s}\right] \Phi_{a u m}=0,
$$

assumindo que exista a inversa de $\left(R+\alpha^{2} \Gamma_{\text {aum }}^{T} P_{s s} \Gamma_{\text {aum }}\right)$.

Portanto, $x_{\text {aum }}[k]$ deve decair com uma taxa de pelo menos $1 / \alpha^{k}$. A relação entre o tempo de acomodação $t_{s}$ e o valor de $\alpha$, como demonstrado em Franklin, Powell e Workman (1997), é dada por:

$$
\left(\frac{1}{\alpha}\right)^{k} \leq 0,01 \Rightarrow \alpha \geq 100^{\frac{1}{k}}=100^{\frac{T_{s}}{t_{s}}}
$$

sendo $T_{s}$ o período de amostragem definido e $t_{s}$ o tempo de acomodação em que os estados de $x_{\text {aum }}[k]$ devem se aproximar do valor de regime estacionário, com um erro menor que $1 \%$.

Em suma, o procedimento de pincer modifica o índice de desempenho (3.20) do controle LQR para (3.23) ao introduzir uma constante $\alpha$ ao problema, que consequentemente também é introduzida no sistema aumentado $\left(\alpha \Phi_{\text {aum }}, \alpha \Gamma_{\text {aum }}\right)$. O índice de desempenho alterado (3.23) é minimizado por um vetor de controle $u[k]$ "ótimo" (3.27), no qual $K_{\text {aum }}$ é dada por (3.28). Este ganho $K_{\text {aum }}$ busca garantir que os estados de $x_{\text {aum }}[k]$ alcancem o valor de regime estacionário dentro do tempo de acomodação $t_{s}$.

\footnotetext{
${ }^{9} P[k]$ e $P[k+1]$ em $(3.19)$ se tornam iguais a $P_{s s}$ em regime estacionário.
} 
Devido ao fato de (3.29) ser quadrática em $P_{s s}$, há mais de uma solução possível, devendo ser escolhida uma solução definida positiva. No programa MATLAB ${ }^{\circledR}$, o comando dlqr fornece uma destas soluções para minimizar (3.20). Sua sintaxe, conforme a notação utilizada nesta dissertação, é dada por:

$$
\left[K_{\_} \text {aum, } P_{-} \text {SS }, E\right]=\operatorname{dlqr}(\text { alpha*Phi_aum, alpha*Gamma_aum, } Q, R) .
$$

Esse comando fornece [K_aum,P_ss,E], que são, respectivamente, a matriz de ganho de regime estacionário K_aum constante, relacionada a matriz P_ss, que é uma das soluções da equação de Riccati na forma estacionária, e os autovalores de malha fechada $E$ do sistema aumentado $\left(\alpha \Phi_{\text {aum }}, \alpha \Gamma_{\text {aum }}\right)$ com o vetor de controle (3.27). 


\section{PROJETOS DOS CONTROLADORES}

Os projetos dos controladores digitais são efetuados com base nas estratégias de controle, apresentadas no Capítulo 3, e nos modelos lineares obtidos em tempo discreto, apresentados na Seção 2.6. São definidas também as especificações de desempenho para cada controlador a ser projetado.

\subsection{Projeto do Controlador PID Digital}

Para o projeto do controlador PID digital, primeiramente realiza-se uma realimentação de velocidade na planta para amortecer a ressonância na frequência de nutação da planta, executada em tempo contínuo para facilitar a demonstração e os cálculos. Com isso, efetua-se a discretização do novo modelo resultante da planta e obtém-se sua matriz de transferência equivalente em tempo discreto, a qual é considerada para o projeto.

\subsubsection{Realimentação de Velocidade na Planta}

Essa realimentação de velocidade é realizada na planta para amortecer a ressonância na frequência de nutação, característica da planta comentada e analisada na Seção F.1, causada por um par de polos complexos conjugados da planta, que, para o caso do modelo desacoplado, estão localizados sobre o eixo imaginário em $s=0 \pm j 19,0054$.

Para isso, uma matriz de realimentação de estados $K_{r v}$ é utilizada, conforme apresentada na Figura 6, para realimentar apenas o estado $\omega_{3}(t)$, relacionado diretamente com a frequência de nutação, no modelo desacoplado em tempo contínuo da planta, composto por (2.25) a (2.28). 
Figura 6: Diagrama de blocos da planta com realimentação de velocidade em tempo contínuo

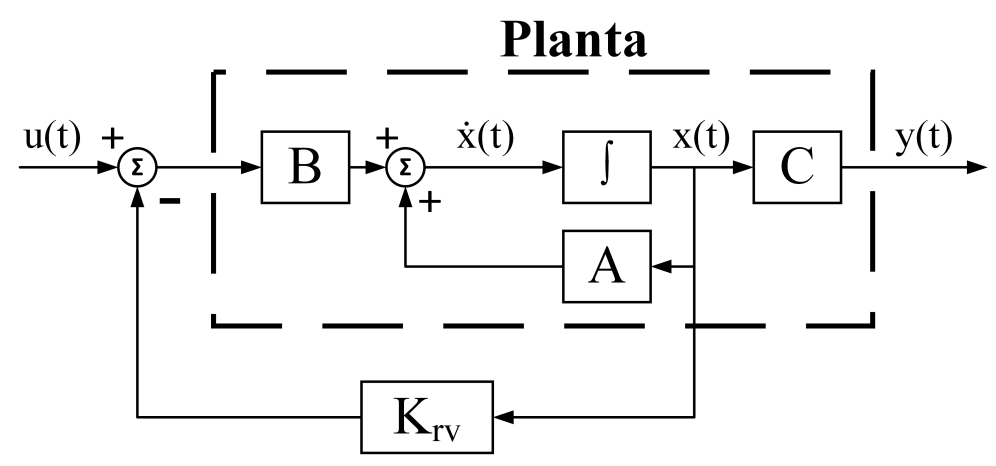

Fonte: Autor.

A nova matriz $A$ com essa realimentação é dada por $A_{v}=A-B K_{r v}$ :

$$
\begin{aligned}
K_{r v}= & {\left[\begin{array}{lllll}
0 & 0 & 0 & 0 & 0 \\
0 & 0 & k_{v} & 0 & 0
\end{array}\right], } \\
A_{v} & =\left[\begin{array}{ccccc}
0 & 0 & 0 & 1 & 0 \\
0 & 0 & 0 & 0 & 1 \\
0 & 0 & -408,1968 k_{v} & 0 & 64,0945 \\
0 & 0 & 0 & 0 & 0 \\
0 & 0 & -5,6355 & 0 & 0
\end{array}\right],
\end{aligned}
$$

sendo que as matrizes $B$ e $C$ permanecem as mesmas neste novo modelo.

A equação característica deste novo modelo $p(s)=\operatorname{det}\left(s I-A_{v}\right)$ é:

$$
p(s)=s^{3}\left(s^{2}+408,1968 k_{v} s+361,2065\right),
$$

na qual o antigo par de polos complexos conjugados puramente imaginários da planta podem ser amortecidos por meio do valor de $k_{v}$.

Definindo $\omega_{n}^{2}=361,2065$ e $2 \zeta \omega_{n}=408,1968 k_{v}$ tem-se que:

$$
k_{v}=\frac{2 \zeta \sqrt{361,2065}}{408,1968}
$$

e, com o intuito de efetuar um bom amortecimento na frequência de nutação, define-se empiricamente $\zeta=0,9665$, para obter $k_{v} \approx 0,09$. 
Portanto, a matriz $A_{v}$ para o $k_{v}$ definido é:

$$
A_{v}=\left[\begin{array}{ccccc}
0 & 0 & 0 & 1 & 0 \\
0 & 0 & 0 & 0 & 1 \\
0 & 0 & -36,7377 & 0 & 64,0945 \\
0 & 0 & 0 & 0 & 0 \\
0 & 0 & -5,6355 & 0 & 0
\end{array}\right]
$$

na qual o antigo par de polos complexos conjugados, que eram puramente imaginários, estão localizados agora em $s=-18,3689 \pm j 4,8777$. Este novo modelo continua sendo controlável e observável e também não possui zeros de transmissão, caracterizando-o como um sistema de fase mínima.

O equivalente discreto, obtido pelo método de discretização ZOH com período de amostragem $T_{s}=0,008$ segundos, é:

$$
\Phi_{v}=\left[\begin{array}{ccccc}
1 & 0 & 0 & 0,0080 & 0 \\
0 & 1 & -0,0002 & 0 & 0,0080 \\
0 & 0 & 0,7358 & 0 & 0,4426 \\
0 & 0 & 0 & 1 & 0 \\
0 & 0 & -0,0389 & 0 & 0,9895
\end{array}\right] \text { e } \Gamma_{v}=\left[\begin{array}{cc}
-0,0012 & 0 \\
0 & -0,0002 \\
0 & 2,8186 \\
-0,3081 & 0 \\
0 & -0,0668
\end{array}\right],
$$

sendo $C$ a mesma matriz do modelo em tempo contínuo. Da mesma forma que nos outros modelos, ele continua sendo controlável e observável, mas o método de discretização inseriu três zeros de transmissão, que não existiam no modelo em tempo contínuo, localizados em $z_{\text {trans }}=\{-0,2488 ;-1 ;-3,4695\}$, caracterizando-o como um sistema de fase não-mínima.

A Figura 7 apresenta o diagrama de blocos do equivalente discreto do novo modelo da planta com a realimentação de velocidade.

Figura 7: Diagrama de blocos da planta com realimentação de velocidade em tempo discreto

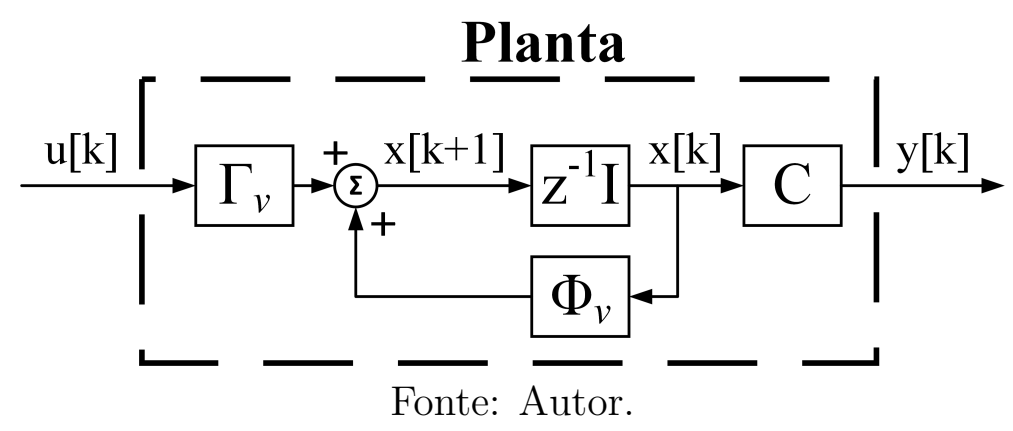


Assim, a matriz de transferência discreta $G_{v}(z)=C\left(z I-\Phi_{v}\right)^{-1} \Gamma_{v}$ deste novo modelo da planta é:

$$
G_{v}(z)=\left[\begin{array}{cc}
G_{v 1}(z) & 0 \\
0 & G_{v 2}(z)
\end{array}\right]
$$

sendo:

$$
\begin{aligned}
& G_{v 1}(z)=-12,32 \times 10^{-4} \frac{(z+1)}{(z-1)^{2}} \\
& G_{v 2}(z)=-1,82 \times 10^{-4} \frac{(z+3,47)(z+0,2488)}{(z-1)\left(z^{2}-1,725 z+0,7453\right)}
\end{aligned}
$$

\subsubsection{Projeto do Controlador}

Para o projeto do controlador PID digital $C(z)$, suas parcelas $C_{1}(z)$ e $C_{2}(z)$ são sintonizadas em relação a (4.8) e (4.9), respectivamente. Porém, como (4.8) e (4.9) são negativos, seus sinais são invertidos para facilitar o projeto, como comentado na Seção 3.1.

Com o intuito de obter amplitudes mínimas de sobressinais nos sinais de saída do sistema, definem-se empiricamente as seguintes especificações de desempenho para o projeto ${ }^{1}$ :

- para a parcela $C_{1}(z):\left\{\begin{array}{l}t_{s_{1}}(5 \%)=t_{s_{\psi_{2}}}(5 \%)=0,3 \text { segundos } \\ M_{p_{1}}(\%)=M_{p_{\psi_{2}}}(\%)=0,63 \%\end{array}\right.$, e

- para a parcela $C_{2}(z):\left\{\begin{array}{l}t_{s_{2}}(5 \%)=t_{s_{\psi_{1}}}(5 \%)=1 \text { segundo } \\ M_{p_{2}}(\%)=M_{p_{\psi_{1}}}(\%)=0,266 \times 10^{-7 \%} \approx 0 \%\end{array}\right.$

para obter os seguintes coeficientes de amortecimento e frequências naturais:

- para a parcela $C_{1}(z): \zeta_{1}=0,85$ e $\omega_{n_{1}}=11,7647$; e

- para a parcela $C_{2}(z): \zeta_{2}=0,99$ e $\omega_{n_{2}}=3,0303$,

que por sua vez, geram os seguintes pares de polos complexos conjugados a serem alocados no plano- $z$ :

- para a parcela $C_{1}(z): z_{0_{1}}=e^{-0,08 \pm j 0,0496}=0,9220 \pm j 0,0457$; e

- para a parcela $C_{2}(z): z_{0_{2}}=e^{-0,024 \pm j 0,0034}=0,9763 \pm j 0,0033$.

\footnotetext{
${ }^{1}$ Lembrando que, como a saída do sistema é $y(t)=\left[\psi_{2}(t) \psi_{1}(t)\right]$, as especificações de desempenho para a parcela $C_{1}(z)$ afetam $\psi_{2}$ e para a parcela $C_{2}(z)$ afetam $\psi_{1}$.
} 
Define-se, empiricamente por meio de simulações, $\alpha_{1}=0,99$ e $\alpha_{2}=0,984 \mathrm{em}$ (3.4), pois tanto (4.8) quanto (4.9) não possuem um polo estável a ser cancelado. Analisando a condição angular $(3.5)$ em $z_{0_{q}}$, obtêm-se $\beta_{q}(q=1,2)$ por meio das seguintes equações:

$$
\begin{aligned}
& \left.\angle G_{v q}(z) C_{q}(z)\right|_{z=z_{0_{q}}}=\pi \Rightarrow \angle z_{0_{q}}-\beta_{q}=\pi-\angle G_{v q}\left(z_{0_{q}}\right)-\angle z_{0_{q}}-\alpha_{q}+\angle z_{0_{q}}+\left\lfloor z_{0_{q}}-1, \mathrm{e}\right. \\
& \left\lfloor z_{0_{q}}-\beta_{q}=\operatorname{atan}\left(\frac{\operatorname{Im}\left(z_{0_{q}}\right)}{\operatorname{Re}\left(z_{0_{q}}\right)-\beta_{q}}\right) \Rightarrow \beta_{q}=\operatorname{Re}\left(z_{0_{q}}\right)-\frac{\operatorname{Im}\left(z_{0_{q}}\right)}{\tan \left(\left\lfloor z_{0_{q}}-\beta_{q}\right)\right.},\right.
\end{aligned}
$$

resultando em $\beta_{1}=0,9531$ e $\beta_{2}=0,9567$.

Ao analisar a condição de módulo (3.6) em $z_{0_{q}}$, obtêm-se $K_{q}$ pela seguinte equação:

$$
K_{q}=\frac{1}{\left|\frac{\left(z_{0_{q}}-\alpha_{q}\right)\left(z_{0_{q}}-\beta_{q}\right)}{z_{0_{q}}\left(z_{0_{q}}-1\right)} G_{v q}\left(z_{0_{q}}\right)\right|},
$$

chegando em $K_{1}=63,5463$ e $K_{2}=45,8557$.

Portanto, os elementos $C_{1}(z)$ e $C_{2}(z)^{2}$ resultantes do controlador projetado são:

$$
\begin{aligned}
& C_{1}(z)=\frac{K_{1}\left(z-\alpha_{1}\right)\left(z-\beta_{1}\right)}{z(z-1)}=-\frac{63,5463(z-0,99)(z-0,9531)}{z(z-1)} \\
& C_{2}(z)=\frac{K_{2}\left(z-\alpha_{2}\right)\left(z-\beta_{2}\right)}{z(z-1)}=-\frac{45,8557(z-0,9843)(z-0,9567)}{z(z-1)}
\end{aligned}
$$

Os polos resultantes de malha fechada da primeira parcela estão localizados em $z=$ $\{0,9902 ; 0,9220+j 0,0457 ; 0,9220-j 0,0457 ; 0,0876\}$, dentre os quais o segundo e o terceiro são o par de polos $z_{0_{1}}$ gerados pelas especificações de desempenho, e o primeiro e o quarto são os outros polos de malha fechada alocados de forma estável, mas fora das especificações de desempenho; e os da segunda parcela em $z=\{0,8865+j 0,2050 ; 0,8865-$ $j 0,2050 ; 0,9763+j 0,0033 ; 0,9763-j 0,0033 ;-0,0086\}$, dentre os quais o terceiro e o quarto são o par de polos $z_{0_{2}}$ gerados pelas especificações de desempenho, e o primeiro, o segundo e o quinto são os outros polos de malha fechada alocados de forma estável, mas fora das especificações de desempenho.

Os gráficos da Figura 8 apresentam o lugar das raízes resultante de cada parcela, sendo o da primeira parcela ilustrada no gráfico (a) e o da segunda parcela no gráfico (b), e suas visões ampliadas, na região do circulo unitário, ilustradas nos gráficos (c) e (d), respectivamente.

\footnotetext{
${ }^{2}$ Relembrando que, para facilitar o projeto de $C_{q}(z)$, foram considerados (4.8) e (4.9) com sinais invertidos no início do projeto, o que causa a inversão de sinal de $K_{q}$ em $C_{q}(z)$, ou seja, na realidade $K_{1}=-63,5463$ e $K_{2}=-45,8557$.
} 
Figura 8: Lugar das raízes do controlador PID

(a) Lugar das Raízes da $1^{a}$ Parcela

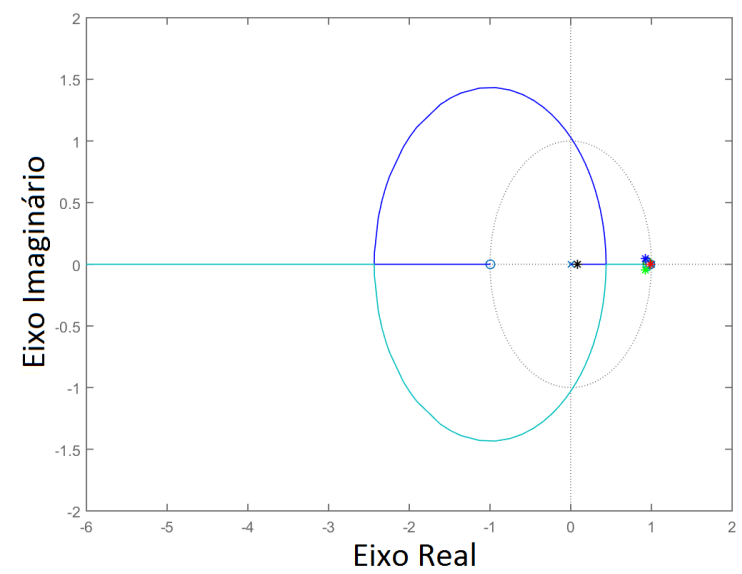

(c) Região do círculo unitário do gráfico (a)

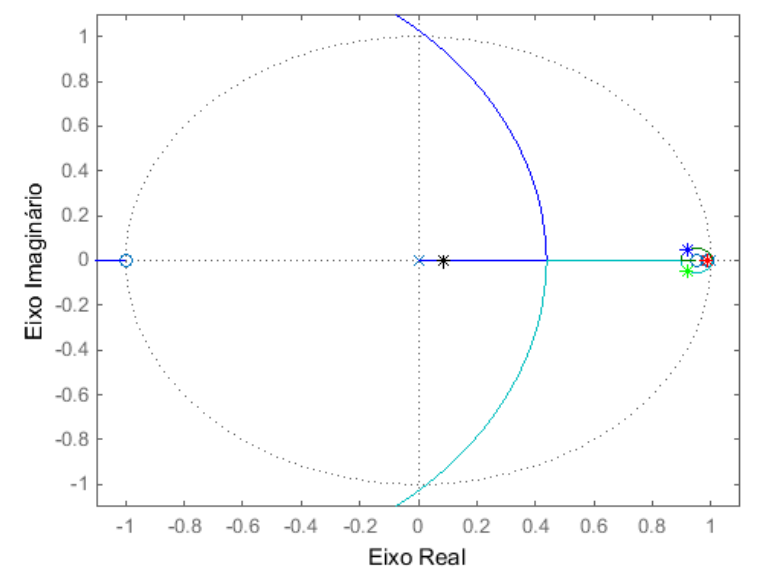

(b) Lugar das Raízes da $2^{a}$ Parcela

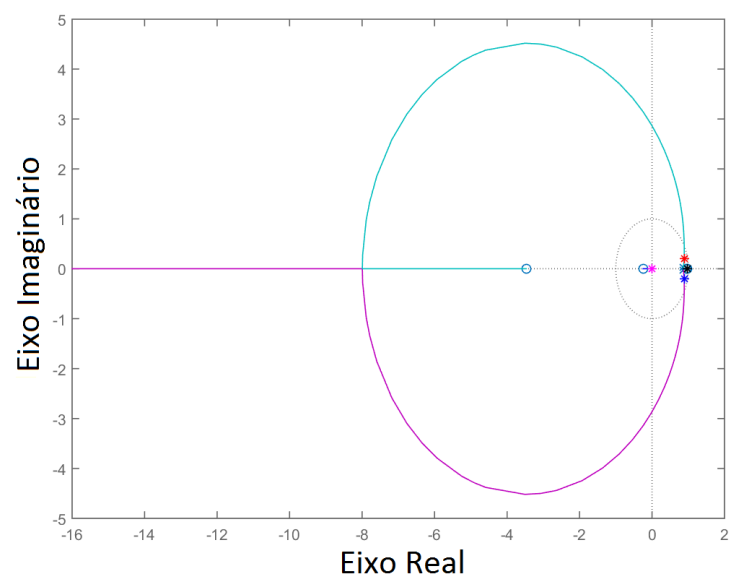

(d) Região do círculo unitário do gráfico (b)

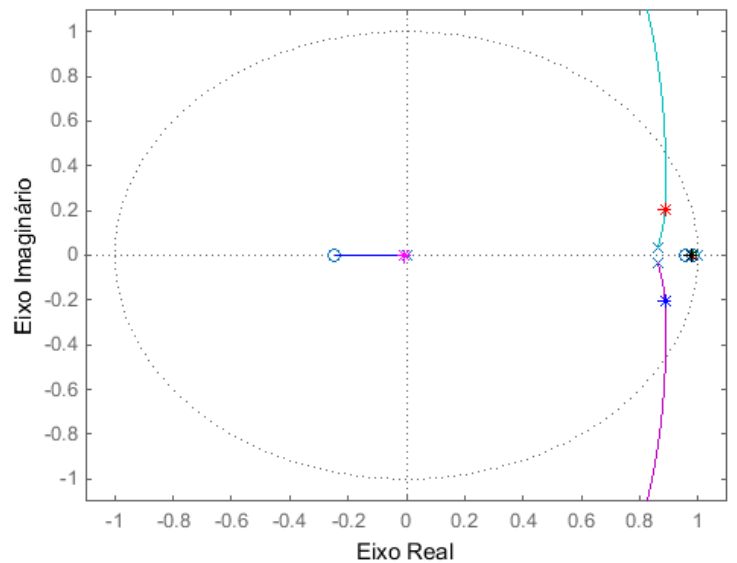

Fonte: Autor.

Esse controlador $C(z)$, projetado com base no modelo desacoplado da planta, é utilizado nas simulações e experimentos tanto para a planta na configuração desacoplada quanto na acoplada de fase mínima. Isso se deve ao fato do controlador, projetado com base no modelo acoplado de fase mínima da planta, ter apresentado resultados piores em relação aos obtidos com base no modelo desacoplado, nos quais os sinais de controle $u[k]$ apresentaram muita oscilação no regime transitório em praticamente todos os testes realizados, o que afetou o desempenho do sistema.

Pode-se também calcular as componentes equivalentes do controlador PID pelas equações (3.10), já considerando que $K_{1}=-63,5463$ e $K_{2}=-45,8557$, que resultam em:

- para a parcela $C_{1}(z): K_{p_{1}}=-3,5698, T_{i_{1}}=0,9587$ e $T_{d_{1}}=0,1344$;

- para a parcela $C_{2}(z): K_{p_{2}}=-2,6594, T_{i_{2}}=0,6822$ e $T_{d_{2}}=0,1299$; 
e substituindo em (3.3) obtêm-se:

$$
\begin{aligned}
& C_{1}(z)=-3,5698\left(1+0,0042 \frac{(z+1)}{(z-1)}+16,8 \frac{(z-1)}{z}\right) \\
& C_{2}(z)=-2,6594\left(1+0,0059 \frac{(z+1)}{(z-1)}+16,2375 \frac{(z-1)}{z}\right) .
\end{aligned}
$$

\subsection{Projeto do Controlador LQR Digital}

O sistema aumentado $\left(\Phi_{\text {aum }}, \Gamma_{\text {aum }}\right)$ equivalente da Figura 5 , para o caso da planta giroscópio tratado nesta dissertação, possuem os seguintes vetores de estados $x_{\text {aum }}[k]$ e de controle $u[k]$ :

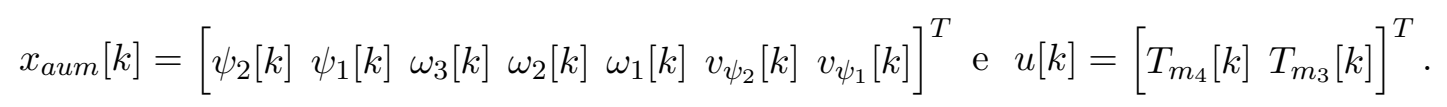

Então, para o projeto do controlador LQR digital, definem-se:

- Frequência de amostragem $f_{s}=125 \mathrm{~Hz} \Rightarrow T_{s}=0,008$ segundos;

- Máxima variação para $\psi_{2}$ e $\psi_{1}=5^{\circ} \approx 0,0873 \mathrm{rad} \approx 7113$ counts (unidades de pulsos de encoder) ${ }^{3}$;

- Máxima variação para $T_{m_{4}}=0,353 \mathrm{~N} . \mathrm{m}$ e $T_{m_{3}}=1,464 \mathrm{~N} \cdot \mathrm{m}^{4}$, o que equivale a $T_{m_{4}}=T_{m_{3}}=16384$ counts (unidades do conversor D/A); e

- Especificação de desempenho (tempo de acomodação para $\psi_{2}$ e $\psi_{1}$ ) como $t_{s}=1$ segundo.

Aplicando a regra de Bryson, obtêm-se as seguintes matrizes $Q$ e $R$ :

$$
Q=1,9767 \cdot 10^{-8} \cdot\left[\begin{array}{ll}
I_{2 x 2} & 0_{2 x 5} \\
0_{5 x 2} & 0_{5 x 5}
\end{array}\right] \text { e } R=3,7253 \cdot 10^{-9} \cdot I_{2 x 2} .
$$

sendo $I$ e 0 as matrizes identidade e nula, respectivamente, com suas devidas dimensões.

Pelo método de pincer, obtém-se:

$$
\alpha \geq 100^{\frac{T_{s}}{t_{s}}}=100^{\frac{0,008}{4}}=1,0375,
$$

\footnotetext{
${ }^{3}$ neste projeto, foram adotados restrições somente para $\psi_{2}=x_{a u m_{1}}$ e $\psi_{1}=x_{a u m_{2}}$. Como os outros elementos $\left(x_{a u m_{3}}\right.$ a $\left.x_{a u m_{7}}\right)$ do vetor de estados do sistema aumentado não foram restringidos para a regra de Bryson, as componentes da diagonal principal da matriz $Q$ resultante, relacionados a esses elementos de $x_{\text {aum }}$, são nulos.

${ }^{4}$ ambos foram definidos com a metade do valor de saturação do torque de seus respectivos motores.
} 
e define-se o menor valor $\alpha=1,0375$.

Dessa forma, aplica-se o comando dlqr do MATLAB ${ }^{\circledR}$ com a sintaxe: [K_aum, P_ss, E] $=\operatorname{dlqr}($ alpha*Phi_aum, alpha*Gamma_aum, $Q, R)$ e obtêm-se as seguintes matrizes de ganhos $K_{\text {aum }}$ de realimentação de estados do sistema aumentado para os casos desacoplado e acoplados:

$$
K_{\text {aum }}=\left[K-K_{i}\right], \text { sendo: }
$$

- para o caso desacoplado:

$$
\begin{aligned}
K & =\left[\begin{array}{ccccc}
-9,5755 & 0 & 0 & -0,8006 & 0 \\
0 & -6,6984 & 0,1056 & 0 & -0,3373
\end{array}\right], \\
K_{i} & =\left[\begin{array}{cc}
-0,3074 & 0 \\
0 & -0,2455
\end{array}\right]
\end{aligned}
$$

- para o caso acoplado de fase mínima:

$$
\begin{aligned}
& K=\left[\begin{array}{ccccc}
-8,9024 & -6,7414 & -0,0328 & -0,7319 & -0,7093 \\
1,4767 & -6,5345 & 0,0928 & 0,0295 & -0,3171
\end{array}\right], \\
& K_{i}=\left[\begin{array}{cc}
-0,2840 & -0,1874 \\
0,0714 & -0,2389
\end{array}\right] \text {; }
\end{aligned}
$$

- para o caso acoplado de fase não-mínima:

$$
\begin{aligned}
K & =\left[\begin{array}{ccccc}
-7,6021 & 8,7429 & -0,0283 & -0,7170 & 0,7387 \\
-3,6941 & -5,4230 & 0,1250 & -0,1150 & -0,3488
\end{array}\right], \\
K_{i} & =\left[\begin{array}{cc}
-0,2299 & 0,2607 \\
-0,1556 & -0,1811
\end{array}\right]
\end{aligned}
$$

sendo $K$ as matrizes de ganhos de realimentação do vetor de estados da planta $(x[k])$ e $K_{i}$ os ganhos dos integradores inseridos $(v[k])$. 


\section{PROCEDIMENTO EXPERIMENTAL}

As simulações, com o modelo não-linear da planta obtido e descrito no Apêndice F, e os experimentos práticos, com a planta física, são realizados por meio de arquivos MATLAB ${ }^{\circledR} /$ Simulink, na qual os arquivos do MATLAB ${ }^{\circledR}$ contêm o algoritmo para gerar os modelos matemáticos, os dados dos controladores e outras informações necessárias para o procedimento, como os sinais de acompanhamento de referência $r(t)$ e de perturbação na saída da planta $d(t)$; e os arquivos Simulink contêm os diagramas de blocos com uma estrutura composta por um bloco do controlador projetado, um bloco da planta, blocos que geram os sinais $r(t)$ e $d(t)$ e blocos que coletam dados gerados no sistema, como os sinais de saída do sistema $y(t)$ e os sinais dos estados da planta $x(t)^{1}$, conforme apresentado de forma simplificada na Figura 9. No Apêndice I estão ilustrados os diagramas de blocos utilizados para os testes realizados, e no Apêndice J estão descritos os algoritmos criados para os controles PID e LQR.

Figura 9: Estrutura do diagrama de blocos dos arquivos Simulink

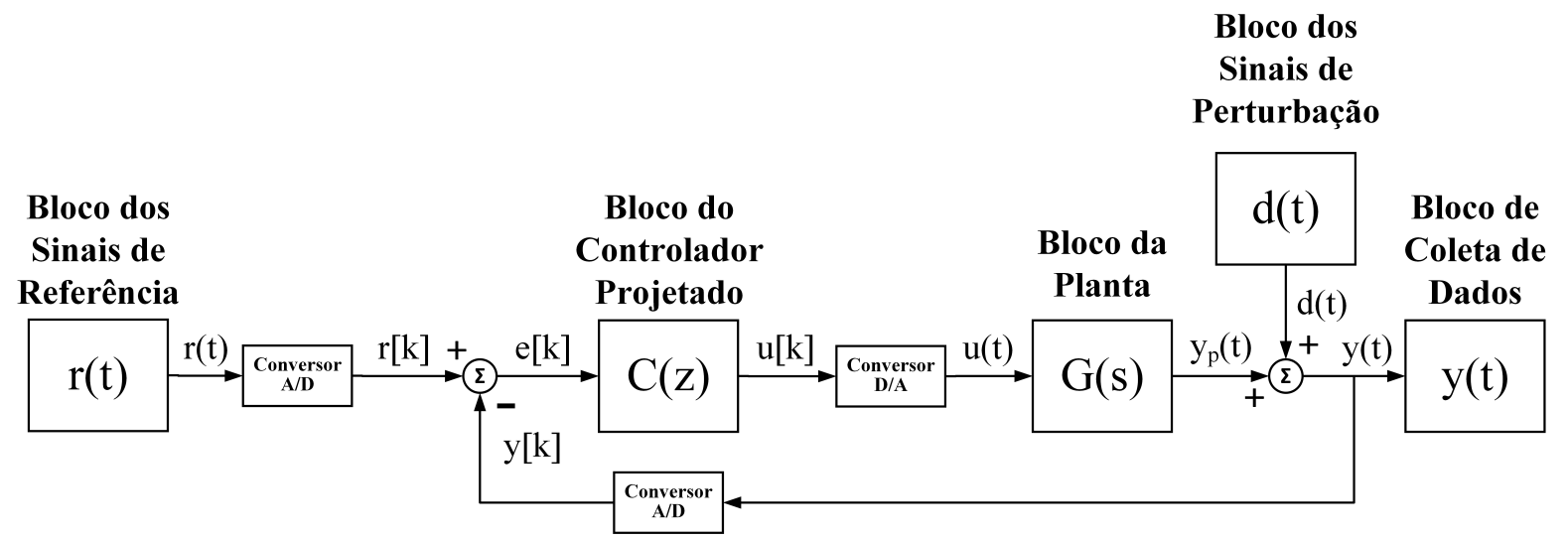

Fonte: Autor.

O bloco da planta é composto por uma S-Function parametrizada por um arquivo MATLAB ${ }^{\circledR}$ com informações para a simulação ou para o experimento prático. No caso da simulação, o arquivo contém o modelo não-linear da planta, descrito no Apêndice F; e no caso do experimento prático, o arquivo é um driver fornecido pela fabricante da planta, que é responsável pela comunicação em tempo real com a planta física.

\footnotetext{
${ }^{1}$ lembrando que os sinais das velocidades angulares dos corpos $\omega_{n}=\dot{\psi}_{n}$ são indiretamente obtidas pelo método retangular para trás (backward retangular rule) a partir dos sinais $\psi_{n}$.
} 
O bloco S-Function, do arquivo de simulação, recebe quatro sinais de entrada: os

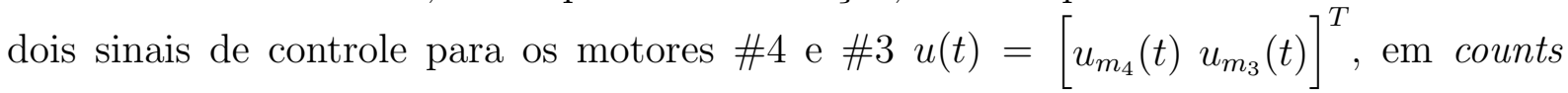
(unidades do conversor D/A), e os dois sinais para acionamento dos freios dos eixos \#2 e \#1; e fornece quatro sinais de saída, que são as posições angulares dos corpos $\psi_{n}(t)$ $(n=1,2,3,4)$, em counts (unidades de pulsos de encoder). O bloco S-Function, do arquivo de experimento, recebe apenas os dois sinais de controle para os motores \#4 e \#3 $(u(t))$ e fornece as mesmas quatro saídas (posições angulares dos corpos $\psi_{n}(t)$ ), que são os sinais fornecidos pelos encoders incrementais $A, B, C$ e $D$ da planta física.

O bloco do controlador PID digital é composto por dois blocos Discrete Transfer Function, onde estão inseridas as equações discretas (4.12) e (4.13) de $C_{1}(z)$ e $C_{2}(z)$, respectivamente, consideradas para os casos de fase mínima; e o bloco do controlador LQR é composto por um bloco Matlab Function, onde estão inseridas as equações de estados dos integradores (3.11) e do vetor de controle (3.12), sendo as matrizes $K$ e $K_{i}$ iguais a (4.19) e (4.20) para o caso desacoplado, (4.21) e (4.22) para o caso acoplado de fase mínima, e (4.23) e (4.24) para o caso acoplado de fase não-mínima.

Para avaliar o controle de atitude da planta, dois tipos de testes são realizados nas simulações e nos experimentos práticos: teste de acompanhamento de referência e teste de rejeição à perturbação na saída da planta. No primeiro teste é avaliado se o controlador projetado é capaz de fazer com que a saída do sistema $y(t)=\left[\psi_{2}(t) \psi_{1}(t)\right]^{T}$ acompanhe o sinal de referência $r(t)=\left[r_{\psi_{2}}(t) r_{\psi_{1}}(t)\right]^{T}$, e no segundo é avaliado se o controlador projetado é capaz de fazer com que o sistema rejeite perturbações $d(t)=\left[d_{\psi_{2}}(t) d_{\psi_{1}}(t)\right]^{T}$ em sua saída. Para isso, três ensaios com sinais diferentes são considerados para $r(t)$ e $d(t)$, tendo $r(t)$ uma amplitude de $15^{\circ}$ e $d(t)$ uma amplitude de $10^{\circ}$ :

Ensaio 1 - pulsos retangulares inseridos separadamente, com durações de 5 segundos cada, sendo que o sinal relacionado a $\psi_{1}\left(r_{\psi_{1}}(t)\right.$ e $\left.d_{\psi_{1}}(t)\right)$ é o primeiro a ser inserido e, após 5 segundos de seu término, é inserido o sinal relacionado a $\psi_{2}\left(r_{\psi_{2}}(t) \text { e } d_{\psi_{2}}(t)\right)^{2}$;

Ensaio 2 - pulsos retangulares inseridos simultaneamente com amplitudes opostas entre si, sendo que primeiramente são inseridos o sinal relacionado a $\psi_{1}\left(r_{\psi_{1}}(t)\right.$ e $\left.d_{\psi_{1}}(t)\right)$ com amplitude positiva e o sinal relacionado a $\psi_{2}\left(r_{\psi_{2}}(t)\right.$ e $\left.d_{\psi_{2}}(t)\right)$ com amplitude negativa, e após 5 segundos do término destes, são inseridos os mesmos sinais, mas com amplitudes invertidas;

\footnotetext{
${ }^{2}$ neste ensaio são considerados tanto o caso com ambos sinais com amplitude positiva, como o caso com amplitude negativa.
} 
Ensaio 3 - sinais senoidais com frequência de 0,4 radianos por segundo, sendo que o sinal relacionado a $\psi_{1}\left(r_{\psi_{1}}(t)\right.$ e $\left.d_{\psi_{1}}(t)\right)$ possui uma defasagem de $\pi / 2$ em relação ao sinal relacionado a $\psi_{2}\left(r_{\psi_{2}}(t)\right.$ e $\left.d_{\psi_{2}}(t)\right)$.

Estes testes são realizados para cada controlador digital projetado, tanto para o caso desacoplado como para o caso acoplado. Eles possuem um tempo de duração total de $t_{\text {run }}=35$ segundos e seguem o seguinte procedimento:

- Após posicionar a planta na "configuração de reset", descrita na Seção 2.2, os sinais dos encoders incrementais são zerados. Em seguida, posiciona-se, primeiramente, os gimbals externo e interno nas posições angulares $\psi_{2_{0}}$ e $\psi_{3_{0}}$, respectivamente, definidas no ponto de operação (2.14) para o caso desacoplado, (2.37) para o caso acoplado de fase mínima, e (2.53) para o caso acoplado de fase não-mínima. O procedimento é iniciado com os freios dos eixos \#1 e \#2 acionados e com um controlador PI simples ativado, que eleva a velocidade do disco e o mantém em $\Omega$ do ponto de operação. Em $t=8$ segundos, os freios são desacionados. Neste instante, a planta está no ponto de operação considerado;

- Em $t=10$ segundos, é desativado o controlador PI simples de velocidade do disco e ativa-se o controlador projetado para o sistema, mantendo a planta no ponto de operação considerado;

- Nesta etapa são inseridos os sinais para cada ensaio considerado acima, sendo $r(t)$ para o teste de acompanhamento de referência e $d(t)$ para o teste de rejeição à perturbação na saída da planta. Nos ensaios 1 e 2 (pulsos retangulares), eles são inseridos em $t=15$ segundos, e no ensaio 3 (sinais senoidais), são inseridos em $t=12$ segundos.

Para ilustrar os sinais dos ensaios, considere o caso desacoplado como exemplo. As Figuras 10 e 11 apresentam os sinais $r(t)$ e $d(t)$, respectivamente, que são inseridos em torno do ponto de operação $\psi_{2_{0}}=\psi_{1}=0^{\circ}$ (caso desacoplado) nos testes de acompanhamento de referência e de rejeição à perturbação na saída da planta, respectivamente, sendo nos gráficos (a) exibidos os pulsos retangulares separados com amplitude positiva do ensaio 1, nos gráficos (b) os pulsos retangulares separados com amplitude negativa também do ensaio 1, nos gráficos (c) os pulsos retangulares simultâneos do ensaio 2, e nos gráficos (d) os sinais senoidais do ensaio 3. E para os casos acoplados de fase mínima e não-mínima, os sinais $r(t)$ e $d(t)$ são inseridos em torno dos seus respectivos pontos de operação $\psi_{2_{0}}$ e $\psi_{1}=0^{\circ}$. 
Figura 10: Gráficos com os sinais $r(t)$ dos ensaios considerados para o teste de acompanhamento de referência

(a) Sinais do ensaio 1 (positivo)

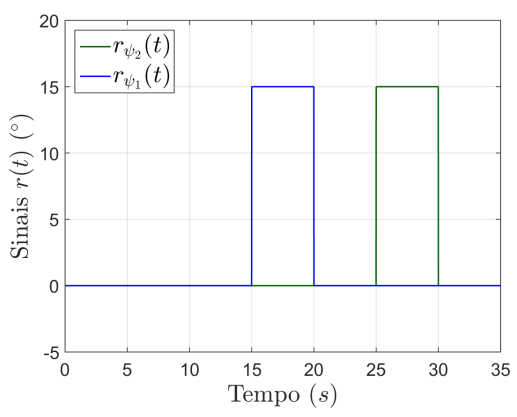

(c) Sinais do ensaio 2

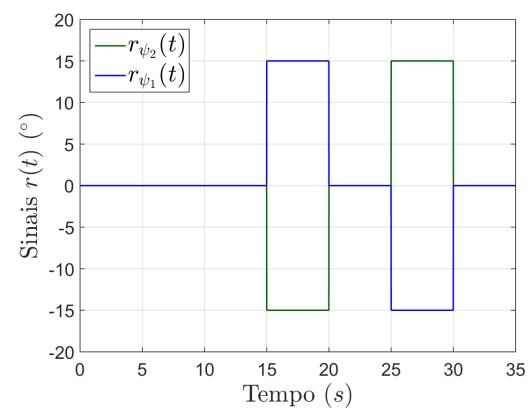

(b) Sinais do ensaio 1 (negativo)

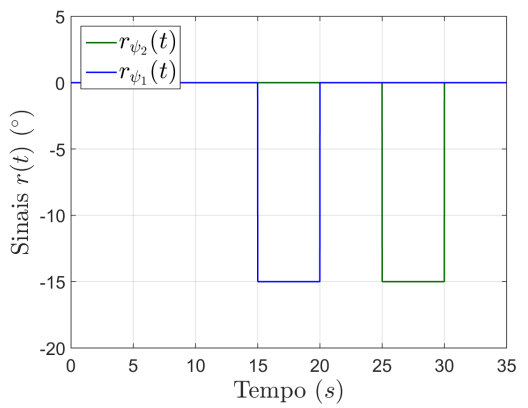

(d) Sinais do ensaio 3

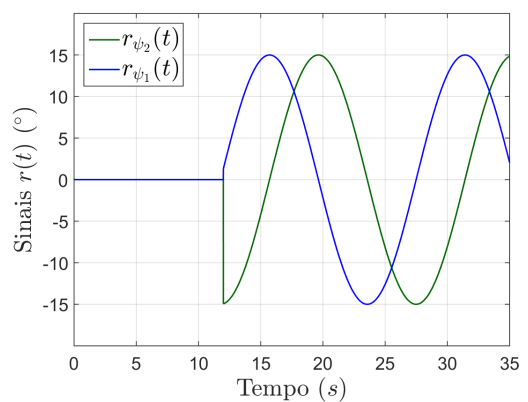

Fonte: Autor.

Figura 11: Gráficos com os sinais $d(t)$ dos ensaios considerados para o teste de rejeição à perturbação na saída da planta

(a) Sinais do ensaio 1 (positivo)

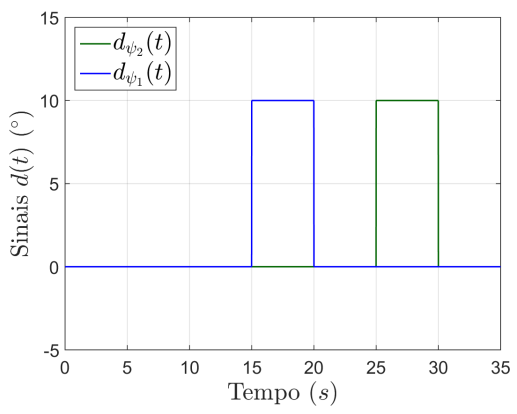

(c) Sinais do ensaio 2

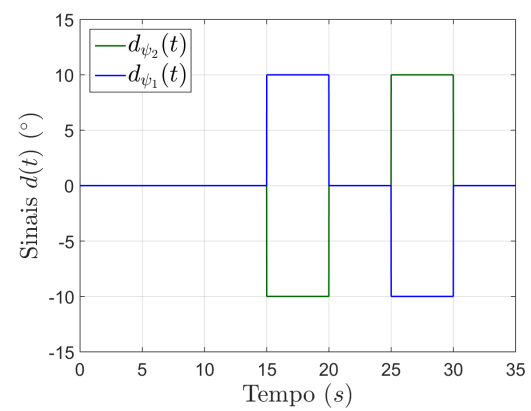

(b) Sinais do ensaio 1 (negativo)

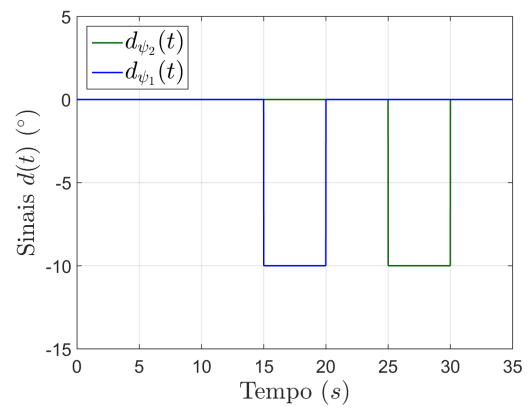

(d) Sinais do ensaio 3

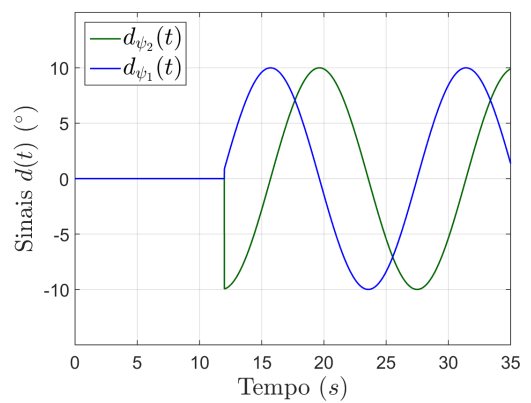

Fonte: Autor. 


\section{ANÁLISE E DISCUSSÃO DOS RESULTADOS}

Neste capítulo, são realizadas a análise e a discussão dos resultados obtidos, apresentados no Apêndice $\mathrm{H}$, nas simulações e nos experimentos práticos dos testes realizados nos sistemas com o controlador projetado (PID ou LQR) e com a planta (na configuração desacoplada ou acoplada, ambas de fase mínima, ou acoplada de fase não-mínima, consideradas nesta dissertação).

Isso é feito em duas partes: primeiro para os casos de fase mínima (casos desacoplado e acoplado de fase mínima), e depois para o caso de fase não-mínima (caso acoplado de fase não-mínima).

\subsection{Casos de Fase Mínima (Desacoplado e Acoplado)}

Vale ressaltar que o controlador PID, projetado com base no modelo desacoplado da planta, foi utilizado em ambos os sistemas com a planta nas configurações desacoplada e acoplada de fase mínima, como informado e justificado na Subseção 4.1.2, enquanto que para o controle LQR foram projetados um controlador para cada caso.

Analisando os resultados obtidos nos casos desacoplado e acoplado de fase mínima, apresentados nas Seções H.1 e H.2, respectivamente, observa-se que ambos os controladores digitais projetados atenderam o objetivo de controle de atitude da planta, tanto nas simulações como nos experimentos práticos, nos quais tanto os sinais de acompanhamento de referência $r(t)$ foram acompanhados, quanto os sinais de perturbação na saída $d(t)$ foram rejeitados, para os 3 ensaios de sinais considerados, com amplitude de $15^{\circ}$ para $r(t)$ e $10^{\circ}$ para $d(t)$.

Os resultados obtidos nos experimentos práticos são bem próximos aos obtidos nas simulações, sendo a diferença mais relevante o fato dos resultados dos experimentos práticos apresentarem "ruído" nos sinais de controle $u(t)$ e nas velocidades angulares $\omega\left(k T_{s}\right)$, mostrando que o fato de algumas dinâmicas não terem sido consideradas no processo de modelagem da planta não afetou, nesses casos de fase mínima, o desempenho dos resultados obtidos nas simulações quando comparados aos obtidos nos experimentos práticos. 
Nos testes de acompanhamento de referência aos pulsos retangulares (ensaios 1 e 2), o controlador PID digital projetado foi capaz de controlar o sistema, mas não atendeu exatamente às especificações de desempenho definidos no projeto, pois foram alocados apenas dois polos de malha fechada com essas especificações em cada parcela, sendo os outros polos alocados de forma estável mas fora das especificações, resultando em sinais de saída $y(t)$ com sobressinais acima das especificações, como pode ser visto nos gráficos (a) e (b) das Figuras 28, 29, 32, 44, 45 e 48 no Apêndice H.

Em contrapartida, os controladores LQR digitais projetados, em relação aos modelos desacoplado e acoplado da planta, apresentaram resultados melhores, atendendo à especificação de desempenho definida nos projetos (tempo de acomodação), e também praticamente não apresentaram sobressinais, sendo que as poucas vezes que apresentaram foram valores muito pequenos em comparação aos apresentados pelo controlador PID, como pode-se observar nos gráficos (a) e (b) das Figuras 36, 37, 40, 52, 53 e 56 no Apêndice H.

Para ilustrar melhor essa análise, a Figura 12 apresenta uma visão ampliada das respostas obtidas, nos experimentos dos testes de acompanhamento de referência com pulsos retangulares simultâneos (ensaio 2), na região inicial do primeiro pulso retangular aplicado (intervalo de tempo entre $t=14$ e $t=19$ segundos), na qual os gráficos da esquerda (a) e (c) apresentam os resultados com o controlador PID e os gráficos da direita (b) e (d) os com os controladores LQR.

Os dados de desempenho dos gráficos da Figura 12 estão relacionados na Tabela 2, dos quais constata-se que os sistemas com o controlador PID realmente apresentaram sinais de saída com sobressinais acima dos especificados no projeto $\left(M_{p_{\psi_{2}}}(\%)=0,63 \%\right.$ e $M_{p_{\psi_{1}}}(\%) \approx$ $0 \%$ ) , como também tempos de acomodação acima dos especificados $\left(t_{s_{\psi_{2}}}(5 \%)=0,3\right.$ segundos e $t_{s_{\psi_{1}}}(5 \%)=1$ segundo) ${ }^{1}$. Já os sistemas com os controladores LQR atenderam o tempo de acomodação adotado no projeto $\left(t_{s}=1\right.$ segundo) em ambos os casos desacoplado e acoplado, nos quais todas as saídas estão dentro da faixa de erro de $1 \%$ (valores entre $\pm 0,15^{\circ}$ ) do valor de regime estacionário a partir dos seus respectivos tempos de acomodação, sendo que somente a saída $\psi_{2}$ do caso desacoplado apresentou um leve sobressinal, quando comparado aos obtidos nos sistemas com o controlador PID, em $t=15,57$ segundos.

\footnotetext{
${ }^{1}$ lembrando que, para o projeto do controlador PID, foi considerado um tempo de acomodação para um erro de $5 \%$, ou seja, uma faixa de $\pm 0,75^{\circ}$ em torno do valor de regime estacionário, pois foram considerados pulsos retangulares com amplitude de $\pm 15^{\circ}$.
} 
Figura 12: Gráficos com a visão ampliada dos sinais de saída obtidos na região inicial do primeiro pulso retangular (intervalo de tempo entre $t=14$ e $t=19$ segundos) aplicado nos experimentos dos testes de acompanhamento de referência com pulsos retangulares simultâneos (ensaio 2), para os sistemas desacoplado [(a) e (b)] e acoplado de fase mínima $[(c)$ e $(d)]$, com os controles PID e LQR, respectivamente

(a) Sistema Desacoplado com PID - visão ampliada da Figura 32b

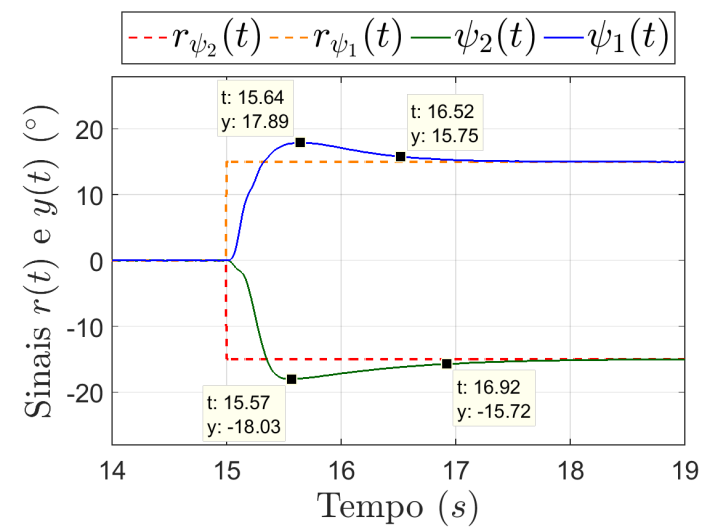

(c) Sistema Acoplado com PID - visão ampliada da Figura 48b
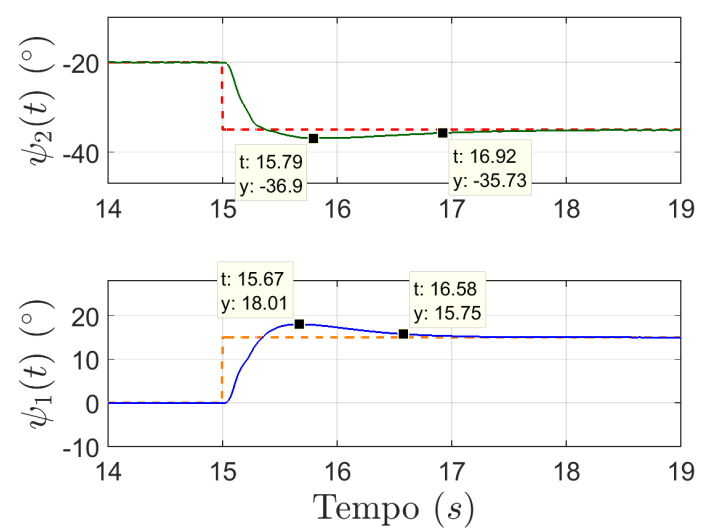

(b) Sistema Desacoplado com LQR - visão ampliada da Figura 40b

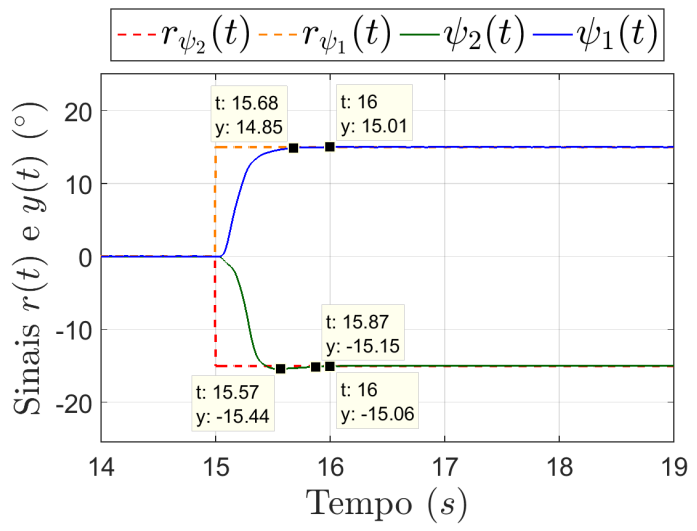

(d) Sistema Acoplado com LQR - visão ampliada da Figura 56b
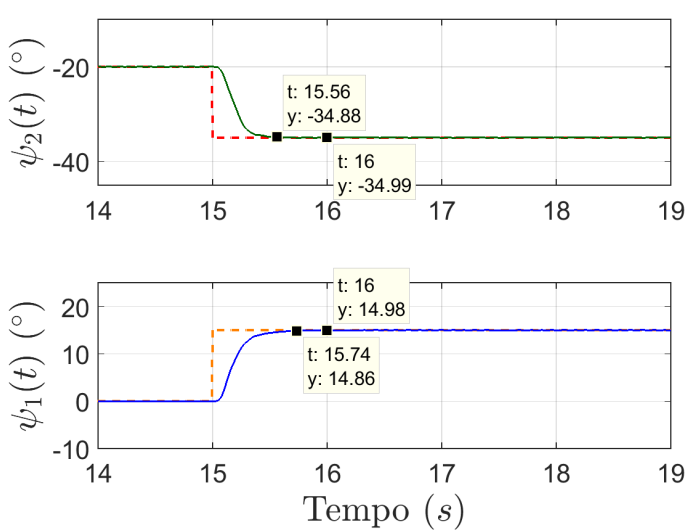

Fonte: Autor.

Tabela 2: Dados de desempenho dos resultados obtidos na região inicial do primeiro pulso retangular dos testes de acompanhamento de referência com pulsos retangulares simultâneos (ensaio 2) - Sistemas de fase mínima (desacoplado e acoplado)

\begin{tabular}{cccccc}
\hline Especificação & Saída & \multicolumn{2}{c}{ PID } & \multicolumn{2}{c}{ LQR } \\
\cline { 3 - 6 } de desempenho & $\boldsymbol{y}$ & Desac. & Acop. & Desac. & Acop. \\
\hline \multirow{2}{*}{$\boldsymbol{t}_{\boldsymbol{s}}$ (em s) } & $\psi_{2}$ & $1,92 \mathrm{~s}$ & $1,92 \mathrm{~s}$ & $0,87 \mathrm{~s}$ & $0,56 \mathrm{~s}$ \\
& $\psi_{1}$ & $1,52 \mathrm{~s}$ & $1,58 \mathrm{~s}$ & $0,68 \mathrm{~s}$ & $0,74 \mathrm{~s}$ \\
\hline \multirow{2}{*}{$\boldsymbol{M}_{\boldsymbol{p}}(\mathrm{em} \%)$} & $\psi_{2}$ & $20,2 \%$ & $12,67 \%$ & $2,93 \%$ & $\approx 0 \%$ \\
& $\psi_{1}$ & $19,27 \%$ & $20,07 \%$ & $\approx 0 \%$ & $\approx 0 \%$ \\
\hline
\end{tabular}

Fonte: Autor. 
Por outro lado, nos testes de acompanhamento de referência com sinais senoidais (ensaio 3), os sistemas com o controlador PID digital, para ambos os casos desacoplado (Figura 34) e acoplado (Figura 50), apresentaram resultados melhores em comparação aos sistemas com os controladores LQR (Figuras 42 e 58, respectivamente), como pode ser visto nos gráficos (a) e (b) dessas figuras, pois os erros de acompanhamento $e(t)=$ $y(t)-r(t)=\left[e_{\psi_{2}} e_{\psi_{1}}\right]^{T}$ foram menores que os dos sistemas com os controladores LQR digitais, como pode-se constatar na Figura 13, na qual os gráficos (a) e (c) exibem os sinais $e(t)$ obtidos nos experimentos com os sistemas contendo o controlador PID digital e a planta nos casos desacoplado e acoplado, respectivamente, e os gráficos (b) e (d) exibem os sinais $e(t)$ obtidos nos experimentos com os sistemas contendo os controladores LQR digitais e a planta nos casos desacoplado e acoplado, respectivamente.

Já nos testes de rejeição à perturbação na saída da planta, ao contrário do que ocorreu no teste de acompanhamento de referência, os sistemas com o controlador PID digital apresentaram melhores resultados nos testes com pulsos retangulares (ensaios 1 e 2), apresentados nos gráficos (a) e (b) das Figuras 30, 31, 33, 46, 47 e 49 no Apêndice H, pois os sinais de saída $y(t)$ dos sistemas com os controladores LQR digitais, embora tenham rejeitado os sinais de perturbação, apresentaram oscilações maiores em ambos os casos desacoplado e acoplado durante o regime transitório, como pode ser visto nos gráficos (a) e (b) das Figuras 38, 39, 41, 54, 55 e 57 no Apêndice H.

Para ilustrar melhor essa análise, de forma análoga ao realizado para o teste de acompanhamento de referência, a Figura 14 apresenta uma visão ampliada das respostas obtidas, nos experimentos dos testes de rejeição à perturbação na saída da planta com pulsos retangulares simultâneos (ensaio 2), na região inicial do primeiro pulso retangular.

Os dados de desempenho dos gráficos da Figura 14 estão relacionados na Tabela 3, dos quais constata-se que os sistemas com o controlador PID continuam apresentando sinais de saída com sobressinais acima dos especificados no projeto $\left(M_{p_{\psi_{2}}}(\%)=0,63 \%\right.$ e $M_{p_{\psi_{1}}}(\%) \approx$ $0 \%$ ) como também tempos de acomodação acima dos especificados $\left(t_{s_{\psi_{2}}}(5 \%)=0,3\right.$ segundos e $t_{s_{\psi_{1}}}(5 \%)=1$ segundo) ${ }^{2}$. Porém, os sistemas com os controladores LQR apresentaram agora oscilações com valores de sobressinais muito altos, e para alguns poucos casos não atenderam o tempo de acomodação adotado no projeto $\left(t_{s}=1\right.$ segundo) dentro da faixa de erro de $1 \%$ (valores entre $\pm 0,1^{\circ}$ ) do valor de regime estacionário, mas ainda são melhores que os apresentados pelo controlador PID.

\footnotetext{
${ }^{2}$ lembrando que, para o projeto do controlador PID, foi considerado um tempo de acomodação para um erro de $5 \%$, ou seja, uma faixa de $\pm 0,5^{\circ}$ em torno do valor de regime estacionário, pois foram considerados pulsos retangulares com amplitude de $\pm 10^{\circ}$.
} 
Figura 13: Gráficos com os sinais de erros de acompanhamento de referência $e(t)$ obtidos nos experimentos dos testes de acompanhamento de referência com sinais senoidais (ensaio 3), na região de regime permanente (intervalo de tempo entre $t=15$ e $t=35$ segundos), para os sistemas desacoplado [(a) e (b)] e acoplado de fase mínima [(c) e (d)], com os controles PID e LQR, respectivamente

(a) Sistema Desacoplado com PID - erro de acompanhamento da Figura 34b

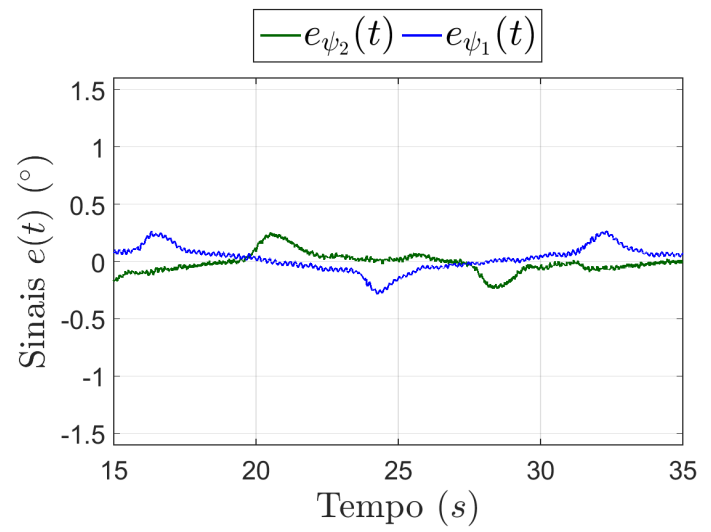

(c) Sistema Acoplado com PID - erro de acompanhamento da Figura 50b

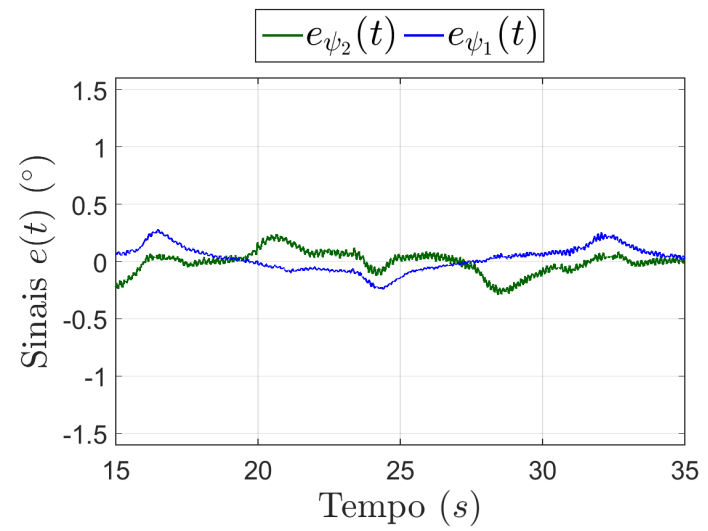

(b) Sistema Desacoplado com LQR - erro de acompanhamento da Figura 42b

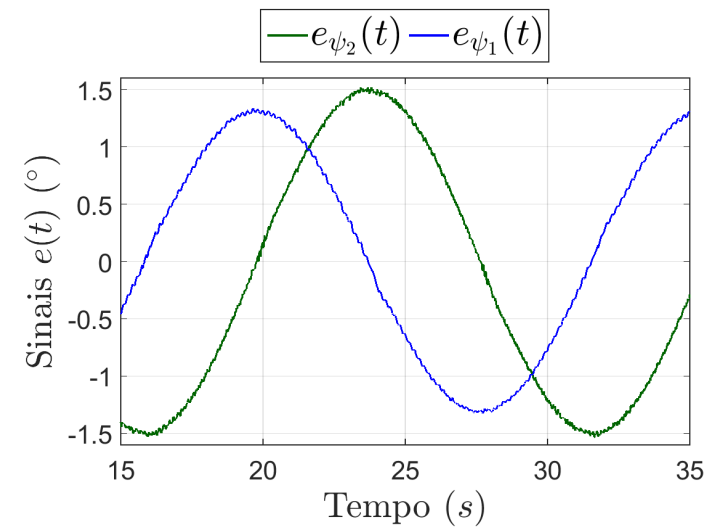

(d) Sistema Acoplado com LQR - erro de acompanhamento da Figura 58b

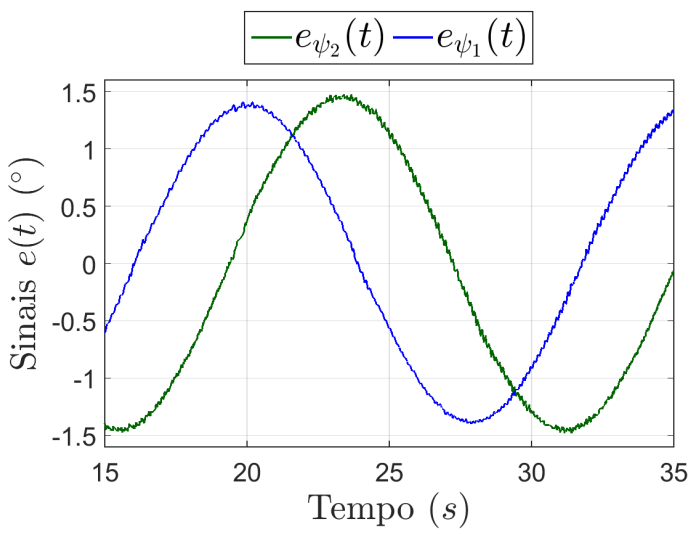

Fonte: Autor.

Tabela 3: Dados de desempenho dos resultados obtidos na região inicial do primeiro pulso retangular dos testes de rejeição à perturbação na saída da planta com pulsos retangulares simultâneos (ensaio 2) - Sistemas de fase mínima (desacoplado e acoplado)

\begin{tabular}{cccccc}
\hline Especificação & Saída & \multicolumn{2}{c}{ PID } & \multicolumn{2}{c}{ LQR } \\
\cline { 3 - 6 } de desempenho & $\boldsymbol{y}$ & Desac. & Acop. & Desac. & Acop. \\
\hline \multirow{2}{*}{$\boldsymbol{t}_{\boldsymbol{s}}$ (em s) } & $\psi_{2}$ & $1,52 \mathrm{~s}$ & $1,49 \mathrm{~s}$ & $1,23 \mathrm{~s}$ & $0,58 \mathrm{~s}$ \\
& $\psi_{1}$ & $1,47 \mathrm{~s}$ & $1,56 \mathrm{~s}$ & $0,78 \mathrm{~s}$ & $0,82 \mathrm{~s}$ \\
\hline \multirow{2}{*}{$\boldsymbol{M}_{\boldsymbol{p}}$ (em \%) } & $\psi_{2}$ & $10,94 \%$ & $8,3 \%$ & $97,39 \%$ & $57,1 \%$ \\
& $\psi_{1}$ & $17,76 \%$ & $18,21 \%$ & $66,8 \%$ & $54,43 \%$ \\
\hline
\end{tabular}

Fonte: Autor. 
Figura 14: Gráficos com a visão ampliada dos sinais de saída obtidos na região inicial do primeiro pulso retangular (intervalo de tempo entre $t=14$ e $t=19$ segundos) aplicado nos experimentos dos testes de rejeição à perturbação na saída da planta com pulsos retangulares simultâneos (ensaio 2), para os sistemas desacoplado [(a) e (b)] e acoplado de fase mínima [(c) e (d)], com os controles PID e LQR, respectivamente

(a) Sistema Desacoplado com PID - visão ampliada da Figura 33b
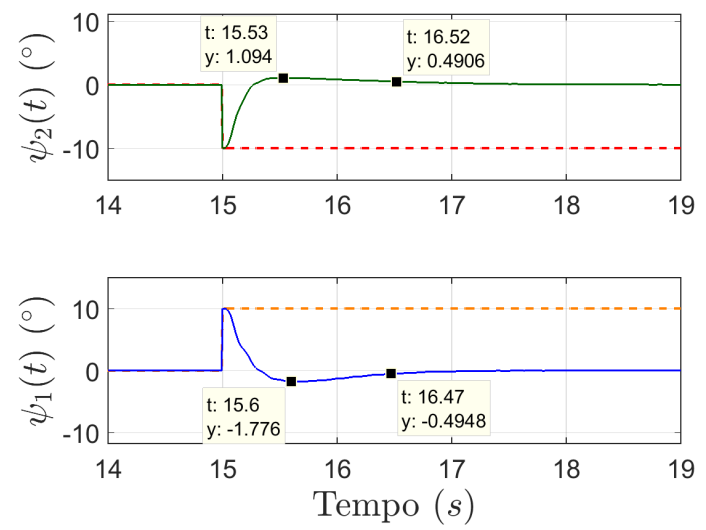

(c) Sistema Acoplado com PID - visão ampliada da Figura 49b
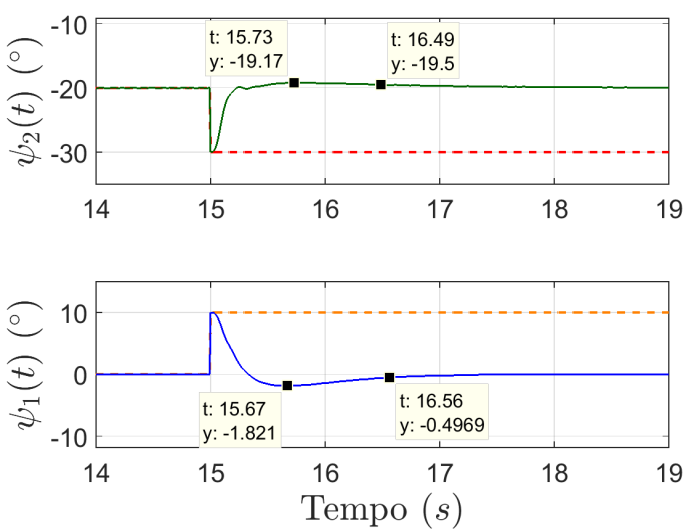

(b) Sistema Desacoplado com LQR - visão ampliada da Figura 41b
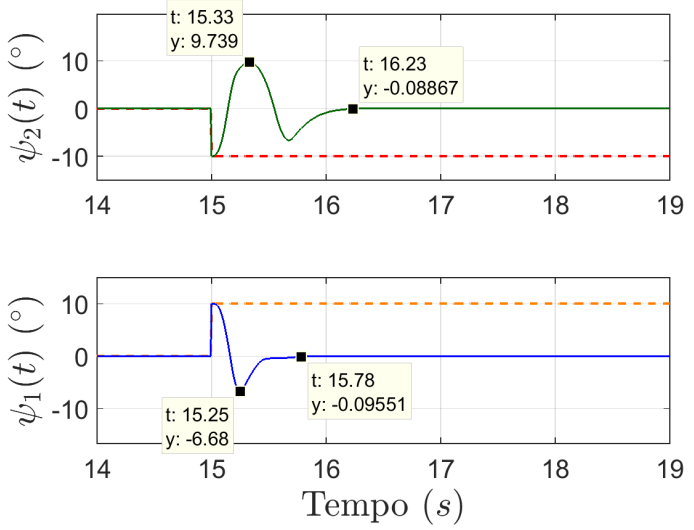

(d) Sistema Acoplado com LQR - visão ampliada da Figura 57b
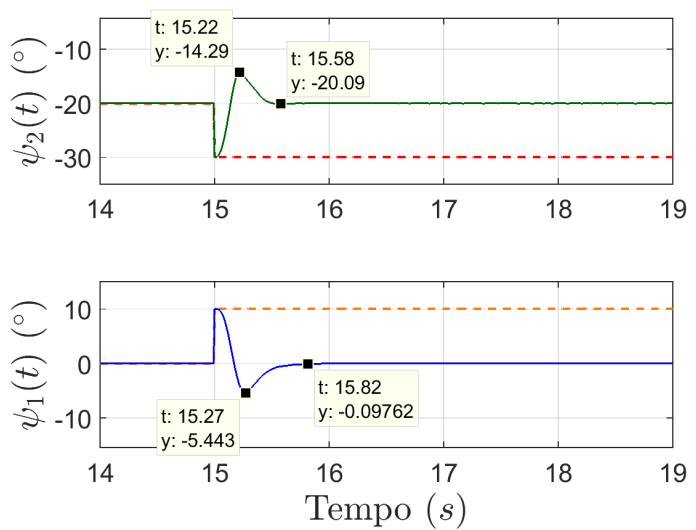

Fonte: Autor.

E, por outro lado, os sistemas com LQR apresentaram resultados melhores nos testes com sinais senoidais (ensaio 3), para ambos os casos desacoplado (Figura 43) e acoplado (Figura 59), em comparação aos sistemas com PID (Figuras 35 e 51, respectivamente), como pode ser visto nos gráficos (a) e (b) dessas figuras, pois os desvios das saídas foram menores que os dos sistemas com o controlador PID, como pode-se constatar na Figura 15, que apresenta uma visão ampliada das respostas obtidas, nos experimentos dos testes de rejeição à perturbação na saída da planta com sinais senoidais (ensaio 3), na região de regime permanente dos sinais de saída $y(t)$, na qual os gráficos da esquerda (a) e (c) apresentam os resultados com o controlador PID e os gráficos da direita (b) e (d) os com os controladores LQR. 
Figura 15: Gráficos com a visão ampliada na região de regime permanente (intervalo de tempo entre $t=15$ e $t=35$ segundos) dos sinais $y(t)$ obtidos nos experimentos dos testes de rejeição à perturbação com sinais senoidais (ensaio 3), para os sistemas desacoplado [(a) e (b)] e acoplado de fase mínima [(c) e (d)], com os controles PID e LQR, respectivamente

(a) Sistema Desacoplado com PID - visão ampliada da Figura 35b

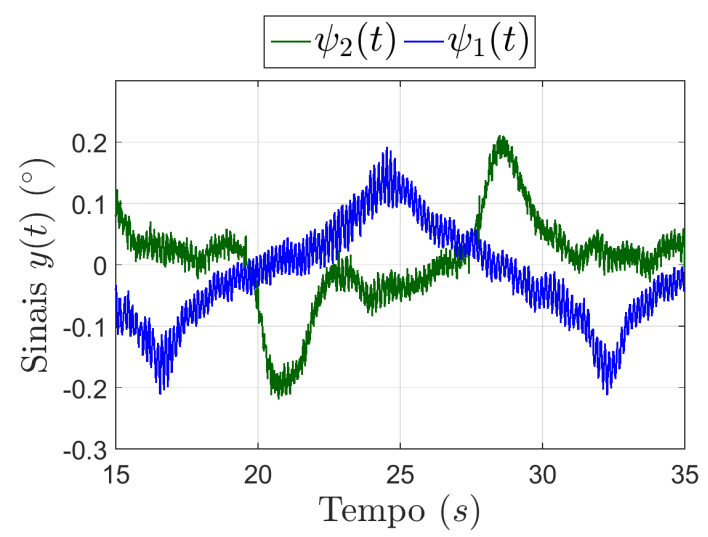

(c) Sistema Acoplado com PID - visão ampliada da Figura 51b
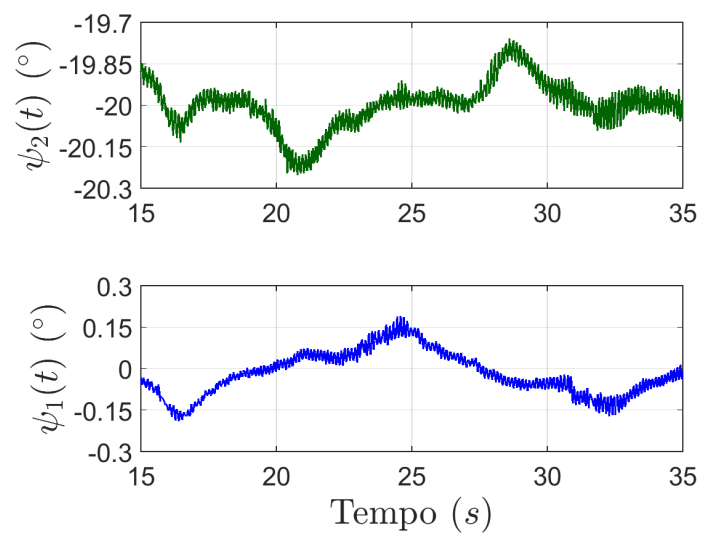

(b) Sistema Desacoplado com LQR - visão ampliada da Figura 43b

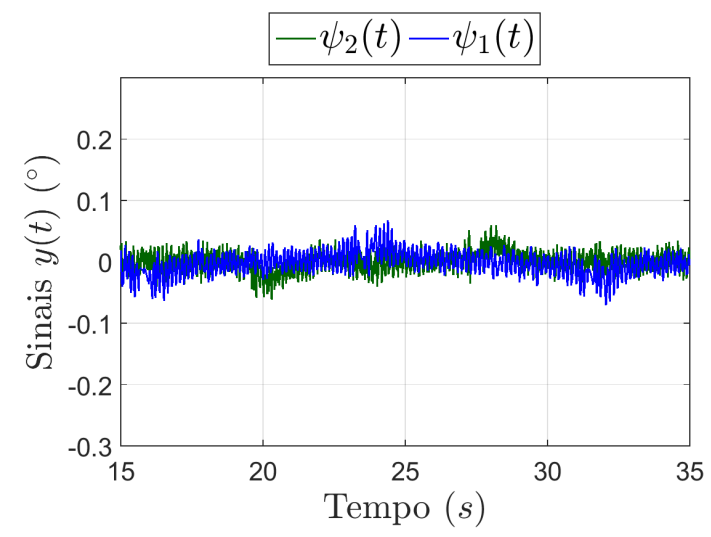

(d) Sistema Acoplado com LQR - visão ampliada da Figura 59b
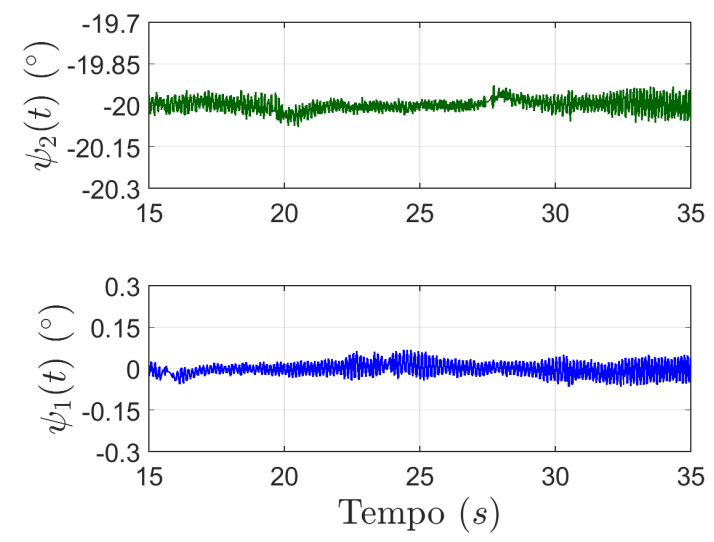

Fonte: Autor.

Os sistemas com LQR apresentaram sinais de controle $u(t)$ com amplitudes menores nos picos em comparação com os sistemas com o controlador PID digital em praticamente todos os ensaios do teste de acompanhamento de referência, como pode-se observar nos gráficos (c) e (d) desses testes.

O controlador PID projetado em relação ao modelo desacoplado da planta apresentou um resultado interessante para o caso desacoplado, no qual o acompanhamento de referência da planta foi atendida mesmo para sinais senoidais com amplitude de $40^{\circ}$, como pode ser visto na Figura 16, na qual observa-se que, mesmo a planta se distanciando bem do ponto de operação $\left(\psi_{2_{0}}=0^{\circ}, \psi_{3_{0}}=0^{\circ}\right.$ e $\left.\Omega=400 R P M\right)$, o acompanhamento do sinal de referência não é afetado neste caso. O mesmo teste foi aplicado ao controlador LQR 
projetado para o caso desacoplado, mas sem sucesso, pois o sistema se torna instável quando é inserido o sinal de referência $r(t)$.

Figura 16: Gráficos com os resultados do teste de acompanhamento de referência com sinais do ensaio 3 com amplitude de $40^{\circ}$ - Sistema desacoplado com controle PID, com os sinais obtidos na simulação [(a), (c) e (e)] e no experimento [(b), (d) e (f)]

$$
\text { (a) Sinais } r(t) \text { e } y(t) \mathrm{em}^{\circ}
$$

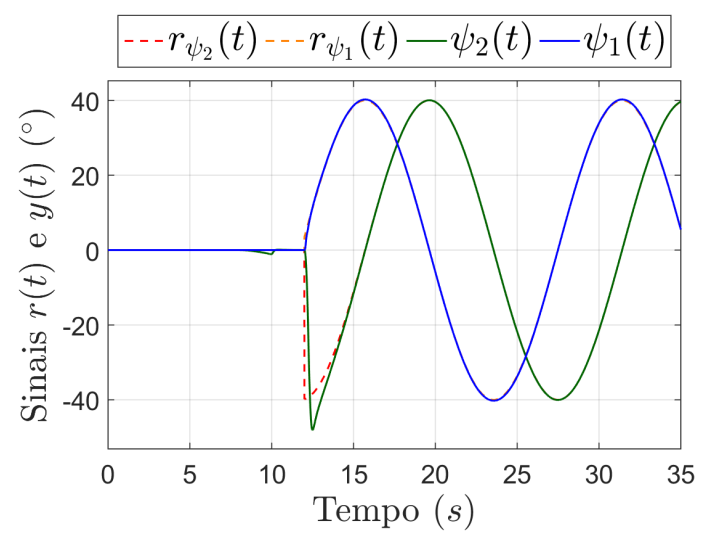

(c) Sinais de Controle $u(t)$ em N.m
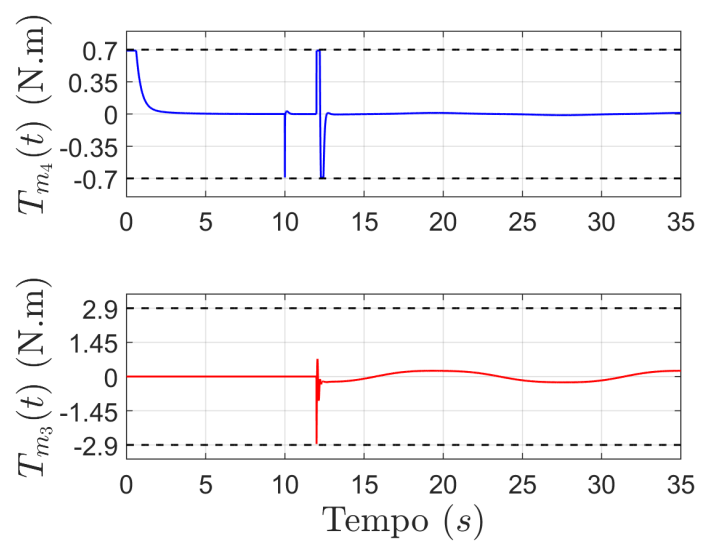

(e) Velocidades Angulares $\omega\left(k T_{s}\right) \mathrm{em}^{\circ} / \mathrm{s}$
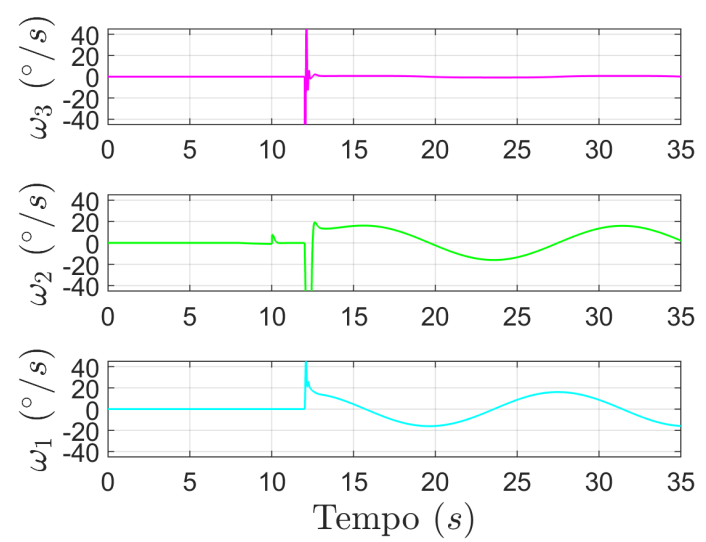

(b) Sinais $r(t)$ e $y(t) \mathrm{em}^{\circ}$

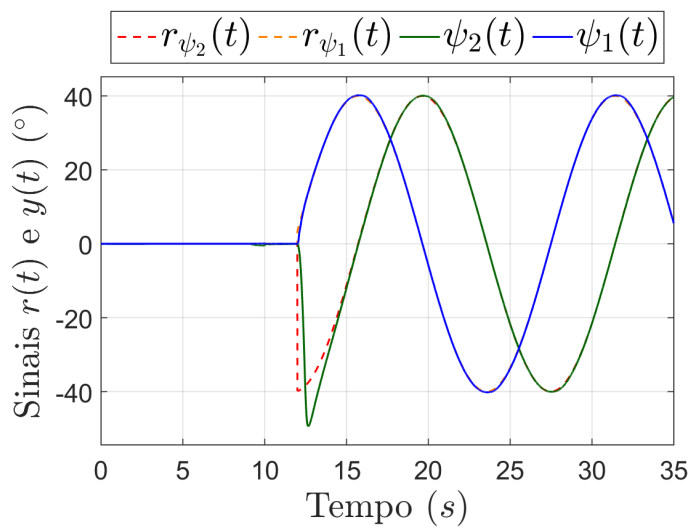

(d) Sinais de Controle $u(t)$ em N.m
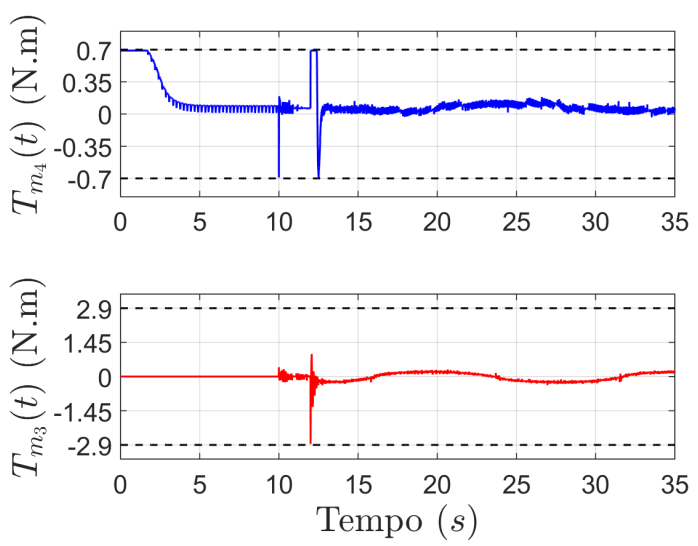

(f) Velocidades Angulares $\omega\left(k T_{s}\right) \mathrm{em} \% \mathrm{~s}$
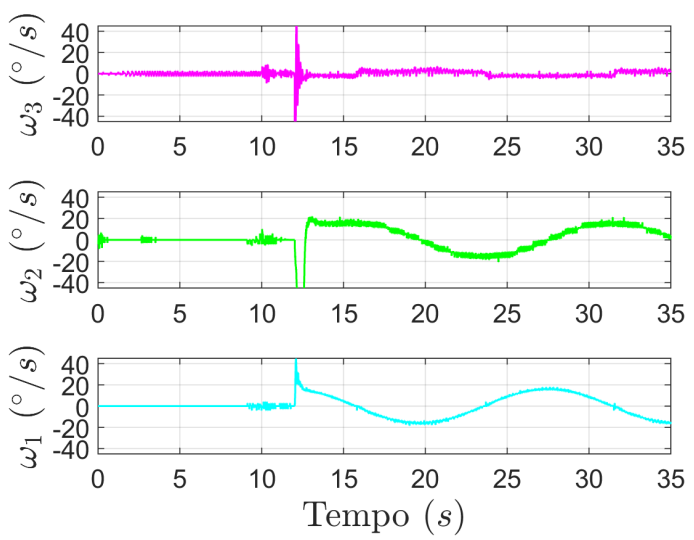

Fonte: Autor. 


\subsection{Caso Acoplado de Fase Não-Mínima}

O controlador PID projetado em relação ao modelo desacoplado da planta não é adequado para este caso, pois a matriz de transferência $G(z)$ do modelo acoplado de fase não-mínima possui um dos elementos da diagonal principal com sinal positivo, enquanto que os dois elementos da diagonal da matriz de transferência do modelo desacoplado possuem ambos sinais negativos, o que impossibilita o uso do controlador projetado em relação ao modelo desacoplado.

Portanto, para o caso acoplado de fase não-mínima, foram realizados ajustes no controlador PID, projetado a partir da diagonal principal do modelo acoplado de fase nãomínima da planta, de forma a obter o controle de atitude da planta, mas não foi possível controlá-la adequadamente.

Por outro lado, o controlador LQR projetado em relação ao modelo acoplado de fase não-mínima atendeu o controle de atitude, no qual alguns dos tempos de acomodação dos sinais de saída $y(t)$ ficaram levemente acima do especificado $\left(\psi_{2}\right.$ no inicio do primeiro pulso e $\psi_{1}$ no final do segundo pulso), e houve pequenos sobressinais nos sinais de saída $y(t)$ em alguns trechos do experimento (no inicio do primeiro pulso e no final do segundo pulso), como pode ser visto no gráfico da Figura 60b, fato que não ocorreu na simulação, ilustrada no gráfico da Figura 60a. Para auxiliar a análise, a Figura 17 apresenta a visão ampliada em cada região dos pulsos retangulares aplicados da Figura 60b e a Tabela 4 apresenta os dados de desempenho em cada uma dessas regiões.

Tabela 4: Dados de desempenho dos resultados obtidos em cada região dos pulsos retangulares aplicados no teste de acompanhamento de referência com pulsos retangulares simultâneos (ensaio 2) - Sistema acoplado de fase não-mínima com o controle LQR

\begin{tabular}{cccccc}
\hline Especificação & Saída & \multicolumn{2}{c}{ Primeiro Pulso } & \multicolumn{2}{c}{ Segundo Pulso } \\
\cline { 3 - 6 } de desempenho & $\boldsymbol{y}$ & Inicial & Final & Inicial & Final \\
\hline \multirow{2}{*}{$\boldsymbol{t}_{\boldsymbol{s}}(\mathrm{em} \mathrm{s})$} & $\psi_{2}$ & $1,03 \mathrm{~s}$ & $0,64 \mathrm{~s}$ & $0,55 \mathrm{~s}$ & $0,98 \mathrm{~s}$ \\
& $\psi_{1}$ & $0,99 \mathrm{~s}$ & $0,72 \mathrm{~s}$ & $0,73 \mathrm{~s}$ & $1,07 \mathrm{~s}$ \\
\hline \multirow{2}{*}{$\boldsymbol{M}_{\boldsymbol{p}}(\mathrm{em} \%)$} & $\psi_{2}$ & $16,79 \%$ & $\approx 0 \%$ & $\approx 0 \%$ & $15,27 \%$ \\
& $\psi_{1}$ & $14,07 \%$ & $\approx 0 \%$ & $\approx 0 \%$ & $14,92 \%$ \\
\hline
\end{tabular}

Fonte: Autor.

Com esses resultados, percebe-se que as simulações com o modelo não-linear obtido nesta dissertação, para este caso de fase não-mínima, apresentam resultados com algumas diferenças em relação aos resultados dos experimentos práticos, o que não ocorreu nos casos de fase mínima. 
Figura 17: Gráficos com a visão ampliada (da Figura 60b) em cada região dos pulsos retangulares aplicados nos experimentos dos testes de acompanhamento de referência com pulsos retangulares simultâneos (ensaio 2) para o sistema acoplado de fase não-mínima com o controle LQR.

(a) Região inicial do primeiro pulso retangular
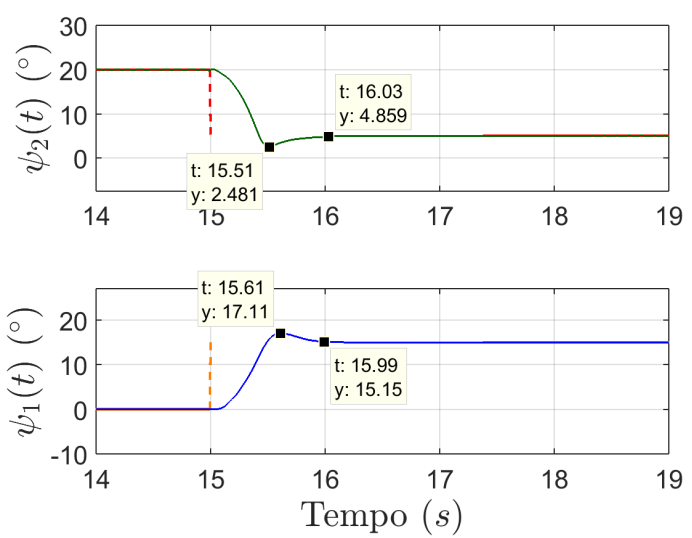

(c) Região inicial do segundo pulso retangular
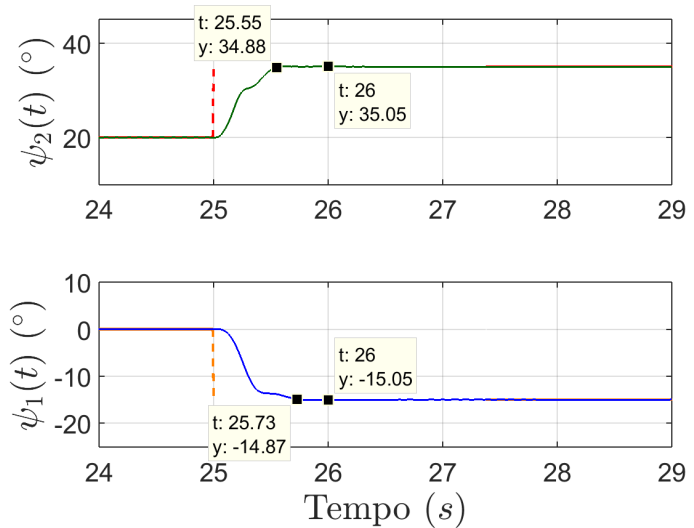

(b) Região final do primeiro pulso retangular
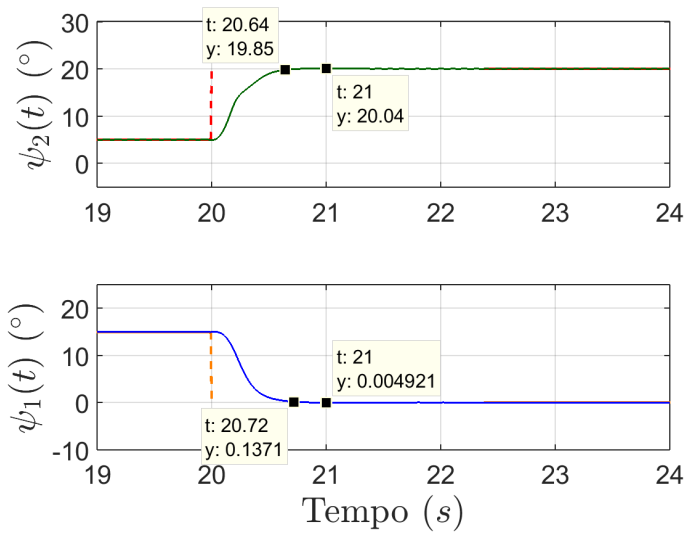

(d) Região final do segundo pulso retangular
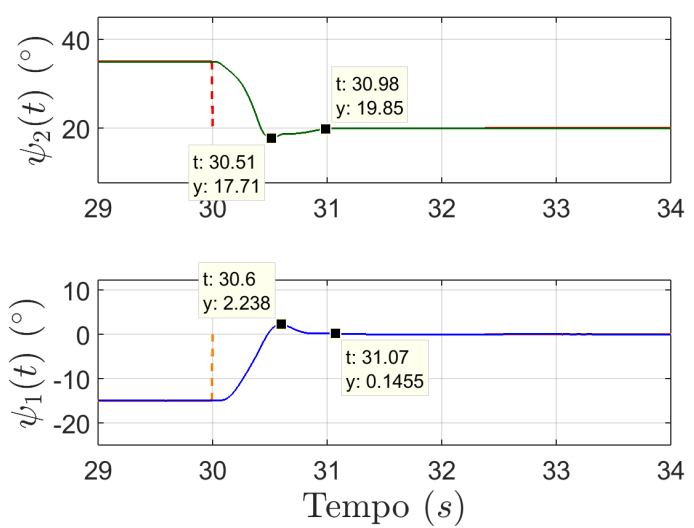

Fonte: Autor. 


\section{CONCLUSÕES E SUGESTÕES DE TRABALHOS FUTUROS}

Conforme a justificativa e a revisão bibliográfica apresentada, conclui-se que o estudo do uso de CMGs como atuadores é importante e bem explorado por pesquisadores. Como são muito utilizados para a estabilização de satélites e veículos espaciais, é importante o desenvolvimento de técnicas de controle digital, em sistemas embarcados, para o controle de atitude. Muitos autores exploram o controle de atitude da planta em tempo contínuo, sendo poucos em tempo discreto, aspecto essencial para o controle digital.

Os resultados obtidos pelos controladores PID e LQR digitais projetados, tanto para a planta na configuração desacoplada de fase mínima (no ponto de operação $\psi_{2_{0}}=0^{\circ}$, $\psi_{3_{0}}=0^{\circ}$ e $\Omega=400 R P M$ ) quanto na configuração acoplada de fase mínima (no ponto de operação $\psi_{2_{0}}=-20^{\circ}, \psi_{3_{0}}=20^{\circ}$ e $\left.\Omega=400 R P M\right)$ mostram que ambos atenderam o objetivo do controle de atitude da planta, como pode ser visto nos resultados obtidos dos testes de acompanhamento de referência e de rejeição à perturbação na saída planta, apresentados nas Seções H.1 e H.2. Estes resultados, ainda que obtidos de testes com sinais de amplitudes pequenas em torno do ponto de operação, de $15^{\circ}$ para o teste de acompanhamento de referência e $10^{\circ}$ para o teste de rejeição à perturbação na saída da planta, são suficientes e válidas, uma vez que os controladores são projetados em relação aos modelos da planta linearizados em torno desses pontos de operação, obtidos na Seção 2.6. Com isso, chegou-se às seguintes conclusões para estes casos de fase mínima:

- Os controladores LQR digitais projetados, tanto em relação ao modelo desacoplado como ao acoplado de fase mínima da planta, apresentaram vantagens nos testes de acompanhamento de referência com pulsos retangulares, nos quais os sinais de saída $y(t)$ atenderam às especificações de desempenho definidas no projeto e praticamente sem sobressinais, sendo muito leve nos poucos casos em que houve; e no teste de rejeição à perturbação na saída com sinais senoidais, no qual os sinais de saída $y(t)$ sofreram um desvio menor. Eles também geraram sinais de controle $u(t)$ com picos de amplitudes menores em praticamente todos os testes de acompanhamento de referência.

- O controlador PID digital projetado em relação ao modelo desacoplado da planta, 
usado tanto para o caso desacoplado como para o caso acoplado de fase mínima, apresentou vantagens no teste de acompanhamento de referencia com sinais senoidais, no qual os sinais de saída $y(t)$ apresentaram um erro de acompanhamento menor; e nos testes de rejeição à perturbação com pulsos retangulares, nos quais os sinais de saída $y(t)$ apresentaram menos oscilações. Ele também possui a vantagem de utilizar apenas os sinais de saída $y(t)$ e a velocidade angular $\omega_{3}\left(k T_{s}\right)$ para a realimentação de velocidade da planta, utilizada para amortecer a sua frequência de nutação; enquanto que os controladores LQR digitais utilizam os cinco estados para a realimentação de estados.

Os resultados obtidos pelos controladores PID e LQR digitais projetados para a planta na configuração acoplada de fase não-mínima (no ponto de operação $\psi_{2_{0}}=20^{\circ}$, $\psi_{3_{0}}=-20^{\circ}$ e $\left.\Omega=400 R P M\right)$ mostram que somente o controlador LQR atendeu o objetivo do controle de atitude da planta, não sendo possível projetar um controlador PID, pela estratégia utilizada nesta dissertação, capaz de atender o objetivo, como descrito na Seção H.3.

Portanto, considerando todos os casos (fases mínima e não-mínima), conclui-se que o uso dos controladores LQR, projetados nesta dissertação, é mais vantajoso pois, mesmo tendo parte dos resultados piores que os obtidos pelo controlador PID, ele foi o único que atendeu o controle de atitude da planta para todos os casos, nos quais atendeu praticamente bem à especificação de desempenho.

Vale ressaltar que essas conclusões são válidas para os controladores digitais projetados com base nas estratégias de controle apresentadas e com as especificações de desempenho definidas nesta dissertação. Se forem utilizadas outras estratégias de controle PID ou LQR, ou definidas outras especificações de desempenho, é bem provável que os controladores digitais projetados obtenham outros resultados que resultem em conclusões diferentes das apresentadas aqui.

Em relação ao modelo não-linear utilizado nas simulações conclui-se que, embora não tenham sido consideradas algumas dinâmicas na modelagem, como o atrito nas juntas da planta, ele foi adequado para avaliar as respostas dos sistemas com os controladores projetados em malha fechada, nos quais os resultados das simulações foram bem próximas dos resultados obtidos nos experimentos práticos.

Como sugestões de trabalhos futuros, pode-se explorar outras técnicas de controle digital, com ênfase em robustez, para o controle de atitude da planta, como as técnicas de controle LQG/LTR (Linear Quadratic Gaussian/Loop Transfer Recovery) e $H_{\infty}$. 


\section{REFERÊNCIAS}

ABBAS, R. S. et al. LPV state-feedback control of a control moment gyroscope. In: AMERICAN CONTROL CONFERENCE, 2013. Conference... [S.l.]: IEEE, 2013. p. 6841-6846.

ARMENISE, M. N. et al. Advances in gyroscope technologies. [S.l.]: Springer Science \& Business Media, 2010.

ASTROM, K. J.; HAGANDER, P.; STERNBY, J. Zeros of sampled systems. In: CONFERENCE ON DECISION AND CONTROL INCLUDING THE SYMPOSIUM ON ADAPTIVE PROCESSES, 19., 1980. Conference... [S.l.]: IEEE, 1980. p. 1077-1081.

BHAT, S.; TIWARI, P. K. Controllability of spacecraft attitude using control moment gyroscopes. IEEE Transactions on Automatic Control, v. 54, n. 3, p. 585-590, March 2009. ISSN 0018-9286.

BROWN, H. B. J.; XU, Y. A single wheel, gyroscopically stabilized robot. IEEE Robotics Automation Magazine, v. 4, n. 3, p. 39-44, Sept 1997. ISSN 1070-9932.

BUTIKOV, E. Precession and nutation of a gyroscope. European Journal of Physics, v. 27, n. 5, p. 1071-1081, 2006. Disponível em: 〈http://stacks.iop.org/0143-0807/27/i= $5 / \mathrm{a}=006\rangle$.

CASTRUCCI, P. B. L.; BITTAR, A.; SALES, R. M. Controle automático. [S.l.]: LTC, 2011.

CHEN, C.-T. Analog and digital control system design: transfer-function, state-space, and algebraic methods. [S.l.]: Saunders College Publishing, 1995.

CRAIG, J. J. Introduction to robotics: mechanics and control. [S.l.]: Addison-Weslg, 2005. v. 3.

DURAND, S. et al. Event-based LQR with integral action. In: EMERGING TECHNOLOGY AND FACTORY AUTOMATION (ETFA), 2014. Proceedings... [S.l.]: IEEE, 2014. p. 1-7.

ECP, T. P. Manual for model 750 Control Moment Gyroscope. [S.1.], 1999.

FADALI M. SAMI; VISIOLI, A. Digital control engineering: analysis and design. 2. ed. [S.1.]: Elsevier, 2013. ISBN 978-1-62198-792-5,978-1-935082-31-6,978-0-12-394391-0.

FRANKLIN, G. F.; POWELL, J. D.; EMAMI-NAEINI, A. Feedback control of dynamics systems. [S.l.]: Pearson Higher Education, 2009. v. 6.

FRANKLIN, G. F.; POWELL, J. D.; WORKMAN, M. L. Digital control of dynamic systems. [S.l.]: Addison-Wesley, 1997. v. 3. 
GAGNE, J. et al. Gyrolock: stabilizing the heart with control moment gyroscope (CMG); from concept to first in vivo assessments. IEEE Transactions on Robotic, v. 28, n. 4, p. 942-954, Aug 2012. ISSN 1552-3098.

HAGIWARA, T.; YUASA, T.; ARAKI, M. Limiting properties of the zeros of sampleddata systems with zero and first-order holds. In: CONFERENCE ON DECISION AND CONTROL, 31., 1992. Conference... [S.l.]: IEEE, 1992. v. 2, p. 1949-1954.

MARDAN, M. F. et al. Robust controller design for attitude control of control moment gyroscope. In: INTERNATIONAL BHURBAN CONFERENCE ON APPLIED SCIENCES AND TECHNOLOGY (IBCAST), 12., 2015. Conference... [S.1.]: IEEE, 2015. p. 163-168.

OGATA, K. Discrete-time control systems. [S.l.]: Prentice Hall Englewood Cliffs, 1995. v. 2.

REYHANOGLU, M.; LOO, J. Van de. State feedback tracking of a nonholonomic control moment gyroscope. In: CONFERENCE ON DECISION AND CONTROL, 45., 2006. Conference... [S.l.]: IEEE, 2006. p. 6156-6161.

SCARBOROUGH, J. B. The gyroscope: theory and application. New York and London: Interscience Publishers, 1958. 257 p.

SCHAUB, J. L. J. H. Analytical Mechanics of Space Systems. 1. ed. [S.l.]: AIAA (American Institute of Aeronautics \& Ast, 2003. (Aiaa Education Series). ISBN 1563475634,9781563475634 .

TEODORESCU, P. P. Mechanical systems, classical models. Mechanics of discrete and continuous systems. 1. ed. [S.1.]: Springer, 2008. v. 2. (Mathematical and Analytical Techniques with Applications to Engineering, v. 2). ISBN 1402089872,9781402089879.

TOWNSEND, N. C.; SHENOI, R. A. Control strategies for marine gyrostabilizers. IEEE Journal of Oceanic Engineering, v. 39, n. 2, p. 243-255, April 2014. ISSN 0364-9059.

YAVUZOGLU, E. et al. Verification of control moment gyroscopes based attitude control systems for agile satellite missions. In: INTERNATIONAL CONFERENCE ON RECENT ADVANCES IN SPACE TECHNOLOGIES (RAST), 5., 2011. Conference... [S.l.]: IEEE, 2011. p. 547-553.

YETKIN, H. et al. Gyroscopic stabilization of an unmanned bicycle. In: AMERICAN CONTROL CONFERENCE (ACC), 2014. Conference... [S.l.]: IEEE, 2014. p. 4549-4554. 


\section{APÊNDICE A - FIXAÇÃO DOS SISTEMAS DE COORDENADAS AOS CORPOS}

A fixação dos sistemas de coordenadas $\{n\}(n=1,2,3,4)$ aos corpos $A, B, C$ e $D$, respectivamente, é realizada pela convenção definida na Seção 3.4 em Craig (2005). Cada sistema de coordenadas é composto por um conjunto ortogonal de três versores $\hat{X}_{n}, \hat{Y}_{n}$ e $\hat{Z}_{n}$, e são numerados a partir da base fixa da planta, na qual o sistema de coordenadas inercial é fixada e recebe, por convenção, o número 0. O sistema de coordenadas do primeiro corpo rígido ligado à base fixa recebe o número 1, e assim por diante, até o sistema de coordenadas do último corpo (neste caso, número 4). Dessa forma, o sistema de coordenadas inercial $\{0\}$ é utilizado como referência para descrever a orientação de todos os corpos da planta.

No caso do giroscópio, as origens de todos os sistemas de coordenadas são adotadas no centro do disco (corpo $D$ ), mas para facilitar a visualização do processo de fixação dos sistemas de coordenadas nas etapas seguintes, seus versores $\hat{X}_{n}, \hat{Y}_{n}$ e $\hat{Z}_{n}$ estão deslocados de suas origens nas Figuras.

Primeiramente, são fixados os versores $\hat{Z}_{n}$, em seus respectivos corpos ${ }^{1}$, na mesma direção dos eixos \#n e com sentido arbitrário, conforme apresentado na Figura 18a. Em seguida, são fixados os versores $\hat{X}_{n}$ direcionados de forma perpendicular ao plano formado pelos versores $\hat{Z}_{n}$ e $\hat{Z}_{n+1}$ e com sentido também arbitrário, com exceção apenas ao versor $\hat{X}_{4}$ que, pelo fato de não existir o versor $\hat{Z}_{5}$, é fixado com a mesma direção e sentido do versor $\hat{X}_{3}$, como ilustrado na Figura 18b. E por fim, os versores $\hat{Y}_{n}$ são direcionados de forma a complementar o sistema ortogonal com orientação positiva, conforme exibido na Figura 18c. O sistema de coordenadas inercial $\{0\}$ é fixado com a mesma orientação do sistema de coordenadas $\{1\}$, completando a fixação de todos os sistemas de coordenadas da planta, como apresenta a Figura 18d.

\footnotetext{
${ }^{1}$ ressaltando que suas origens são fixadas no centro do disco (corpo $\left.D\right)$.
} 
Figura 18: Etapas da fixação dos sistemas de coordenadas aos corpos da planta

(a) Fixação dos versores $\hat{Z}_{n}$

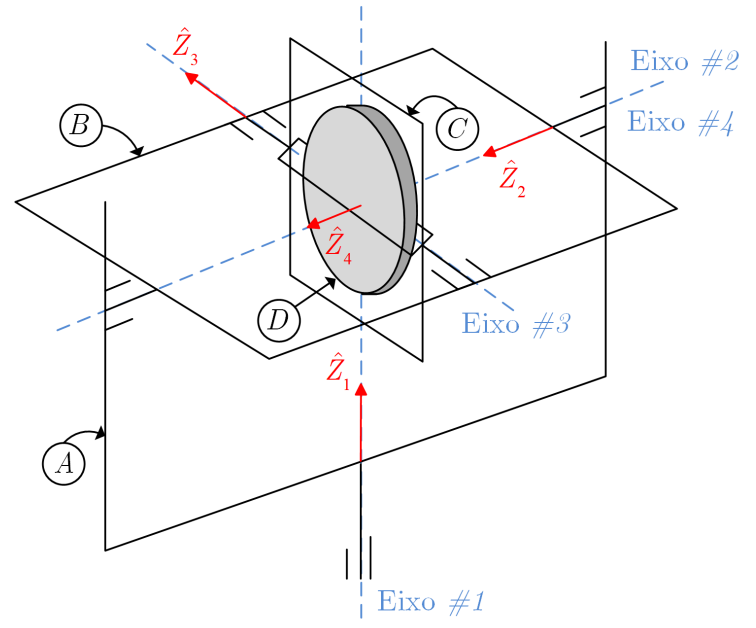

(c) Fixação dos versores $\hat{Y}_{n}$

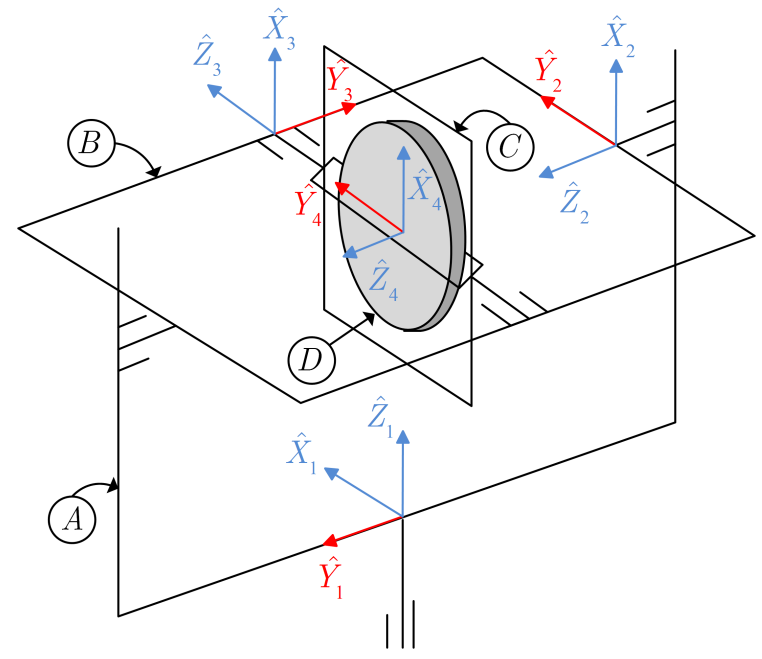

(b) Fixação dos versores $\hat{X}_{n}$

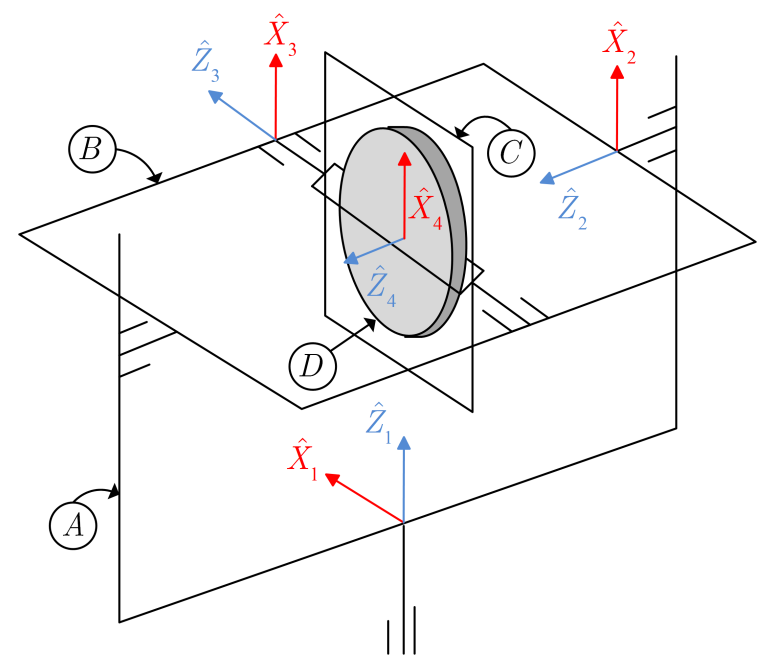

(d) Sistemas de coordenadas resultantes

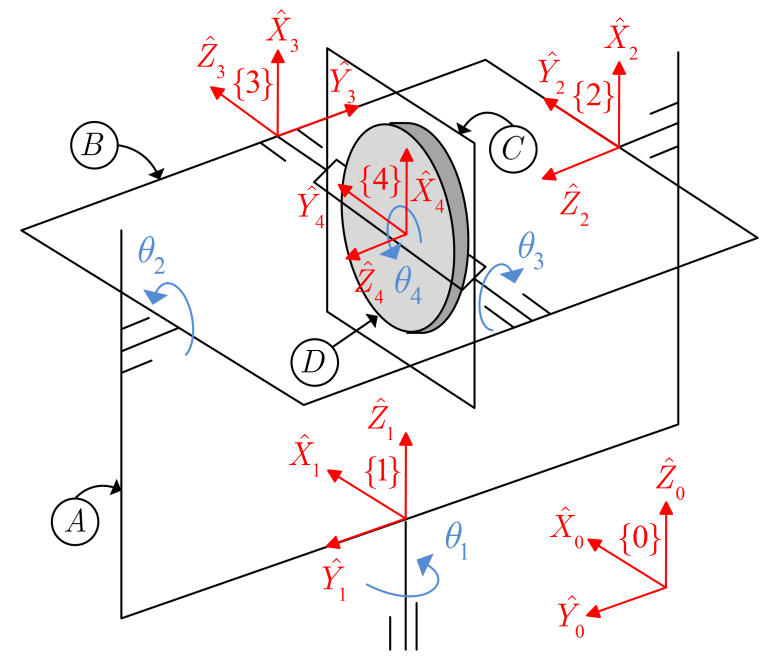

Fonte: Autor.

Com os sistemas de coordenadas fixados em seus respectivos corpos, definem-se as variáveis de junta $\theta_{n}$ como sendo os ângulos formados entre os versores $\hat{X}_{n}$ e $\hat{X}_{n-1}$, sendo nulo quando estes versores estão sobrepostos um ao outro, e adotado como positivo quando $\hat{X}_{n}$ é rotacionado pela regra da mão direita, em torno do eixo $\hat{Z}_{n}$, em relação a $\hat{X}_{n-1}$, conforme ilustrado também na Figura 18d. 


\section{APÊNDICE B - VETORES DE VELOCIDADE ANGULAR}

Por causa da convenção utilizada para a fixação dos sistemas de coordenadas $\{n\}$ aos corpos da planta, pode-se calcular a velocidade de cada corpo ordenadamente, começando pela base fixa da planta. Então, a velocidade angular total do corpo atrelado a $\{n\}$ será a sua própria velocidade angular somado à velocidade angular total do corpo atrelado a $\{n-1\}$. Com essas relações, é possível descrever a velocidade angular de um corpo da planta em relação ao próprio sistema de coordenadas atrelado a ele, que é denominada vetor de velocidade angular e recebe a notação ${ }^{n} \omega_{n}(n=1,2,3,4)$. Esse vetor é obtido por meio da seguinte formulação iterativa, demonstrada na Seção 5.6 em Craig (2005):

$$
{ }^{n} \omega_{n}={ }_{n-1}^{n} R \cdot{ }^{n-1} \omega_{n-1}+\dot{\psi}_{n} \cdot{ }^{n} \hat{Z}_{n},
$$

sendo ${ }_{n-1}^{n} R$ as matrizes de rotação, cuja a definição e os cálculos são apresentados no Apêndice C; $\dot{\psi}_{n}=\omega_{n}$ e o vetor ${ }^{n} \hat{Z}_{n}=\left[\begin{array}{lll}0 & 0 & 1\end{array}\right]^{T}$.

Por convenção ${ }^{0} \omega_{0}=\left[\begin{array}{lll}0 & 0 & 0\end{array}\right]^{T}$, pois a orientação do sistema inercial $\{0\}$ não se altera em nenhum instante de tempo.

\section{B.1 Cálculo de ${ }^{1} \omega_{1}$}

Para $n=1$, tem-se que:

$$
\begin{gathered}
{ }^{1} \omega_{1}={ }_{0}^{1} R \cdot{ }^{0} \omega_{0}+\dot{\psi}_{1} \cdot{ }^{1} \hat{Z}_{1}={ }_{0}^{1} R \cdot\left[\begin{array}{l}
0 \\
0 \\
0
\end{array}\right]+\omega_{1} \cdot\left[\begin{array}{l}
0 \\
0 \\
1
\end{array}\right] \Rightarrow \\
{ }^{1} \omega_{1}=\left[\begin{array}{l}
0 \\
0 \\
\omega_{1}
\end{array}\right] .
\end{gathered}
$$




\section{B.2 Cálculo de ${ }^{2} \omega_{2}$}

Considerando $n=2$, resulta em:

$$
\begin{aligned}
{ }^{2} \omega_{2}={ }_{1}^{2} R \cdot{ }^{1} \omega_{1}+\dot{\psi}_{2} \cdot{ }^{2} \hat{Z}_{2}= & {\left[\begin{array}{ccc}
\sin \left(\psi_{2}\right) & 0 & \cos \left(\psi_{2}\right) \\
\cos \left(\psi_{2}\right) & 0 & -\sin \left(\psi_{2}\right) \\
0 & 1 & 0
\end{array}\right] \cdot\left[\begin{array}{l}
0 \\
0 \\
\omega_{1}
\end{array}\right]+\omega_{2} \cdot\left[\begin{array}{l}
0 \\
0 \\
1
\end{array}\right] \Rightarrow } \\
{ }^{2} \omega_{2} & =\left[\begin{array}{c}
\cos \left(\psi_{2}\right) \omega_{1} \\
-\sin \left(\psi_{2}\right) \omega_{1} \\
\omega_{2}
\end{array}\right] .
\end{aligned}
$$

\section{B.3 Cálculo de ${ }^{3} \omega_{3}$}

Com $n=3$, obtém-se:

$$
\begin{gathered}
{ }^{3} \omega_{3}={ }_{2}^{3} R \cdot{ }^{2} \omega_{2}+\dot{\psi}_{3} \cdot{ }^{3} \hat{Z}_{3}=\left[\begin{array}{ccc}
\cos \left(\psi_{3}\right) & 0 & -\sin \left(\psi_{3}\right) \\
-\sin \left(\psi_{3}\right) & 0-\cos \left(\psi_{3}\right) \\
0 & 1 & 0
\end{array}\right] \cdot\left[\begin{array}{c}
\cos \left(\psi_{2}\right) \omega_{1} \\
-\sin \left(\psi_{2}\right) \omega_{1} \\
\omega_{2}
\end{array}\right]+\left[\begin{array}{c}
0 \\
0 \\
\omega_{3}
\end{array}\right] \Rightarrow \\
{ }^{3} \omega_{3}=\left[\begin{array}{c}
\cos \left(\psi_{3}\right) \cos \left(\psi_{2}\right) \omega_{1}-\sin \left(\psi_{3}\right) \omega_{2} \\
-\sin \left(\psi_{3}\right) \cos \left(\psi_{2}\right) \omega_{1}-\cos \left(\psi_{3}\right) \omega_{2} \\
-\sin \left(\psi_{2}\right) \omega_{1}+\omega_{3}
\end{array}\right] .
\end{gathered}
$$

\section{B.4 Cálculo de ${ }^{4} \omega_{4}$}

E por fim, para $n=4$, tem-se que:

$$
\begin{aligned}
{ }^{4} \omega_{4} & ={ }_{3}^{4} R \cdot{ }^{3} \omega_{3}+\dot{\psi}_{4} \cdot{ }^{4} \hat{Z}_{4}= \\
& =\left[\begin{array}{ccc}
\cos \left(\psi_{4}\right) & 0 & \sin \left(\psi_{4}\right) \\
-\sin \left(\psi_{4}\right) & 0 & \cos \left(\psi_{4}\right) \\
0 & -1 & 0
\end{array}\right] \cdot\left[\begin{array}{c}
\cos \left(\psi_{3}\right) \cos \left(\psi_{2}\right) \omega_{1}-\sin \left(\psi_{3}\right) \omega_{2} \\
-\sin \left(\psi_{3}\right) \cos \left(\psi_{2}\right) \omega_{1}-\cos \left(\psi_{3}\right) \omega_{2} \\
-\sin \left(\psi_{2}\right) \omega_{1}+\omega_{3}
\end{array}\right]+\left[\begin{array}{l}
0 \\
0 \\
\omega_{4}
\end{array}\right] \Rightarrow \\
{ }^{4} \omega_{4}= & {\left[\begin{array}{c}
\cos \left(\psi_{4}\right) \cos \left(\psi_{3}\right) \cos \left(\psi_{2}\right) \omega_{1}-\cos \left(\psi_{4}\right) \sin \left(\psi_{3}\right) \omega_{2}-\sin \left(\psi_{4}\right) \sin \left(\psi_{2}\right) \omega_{1}+\sin \left(\psi_{4}\right) \omega_{3} \\
-\sin \left(\psi_{4}\right) \cos \left(\psi_{3}\right) \cos \left(\psi_{2}\right) \omega_{1}+\sin \left(\psi_{4}\right) \sin \left(\psi_{3}\right) \omega_{2}-\cos \left(\psi_{4}\right) \sin \left(\psi_{2}\right) \omega_{1}+\cos \left(\psi_{4}\right) \omega_{3} \\
\sin \left(\psi_{3}\right) \cos \left(\psi_{2}\right) \omega_{1}+\cos \left(\psi_{3}\right) \omega_{2}+\omega_{4}
\end{array}\right] . }
\end{aligned}
$$




\section{APÊNDICE C - MATRIZES DE ROTAÇÃO}

As matrizes de rotação ${ }_{n}^{n-1} R(n=1,2,3,4)$ descrevem a orientação dos versores do sistema de coordenadas $\{n\}$ em relação ao sistema $\{n-1\}$. Isso é feito escrevendo os versores $\hat{X}_{n}, \hat{Y}_{n}$ e $\hat{Z}_{n}$ do sistema de coordenadas $\{n\}$ em termos do sistema de coordenadas $\{n-1\}$, definidos com as notações: ${ }^{n-1} \hat{X}_{n},{ }^{n-1} \hat{Y}_{n}$ e ${ }^{n-1} \hat{Z}_{n}$, respectivamente. Colocando estes vetores lado a lado como colunas de uma matriz 3x3 obtêm-se as matrizes de rotação:

$$
{ }_{n}^{n-1} R=\left[{ }^{n-1} \hat{X}_{n}{ }^{n-1} \hat{Y}_{n}{ }^{n-1} \hat{Z}_{n}\right]=\left[\begin{array}{cccc}
\hat{X}_{n} \cdot \hat{X}_{n-1} & \hat{Y}_{n} \cdot \hat{X}_{n-1} & \hat{Z}_{n} \cdot \hat{X}_{n-1} \\
\hat{X}_{n} \cdot \hat{Y}_{n-1} & \hat{Y}_{n} \cdot \hat{Y}_{n-1} & \hat{Z}_{n} \cdot \hat{Y}_{n-1} \\
\hat{X}_{n} \cdot \hat{Z}_{n-1} & \hat{Y}_{n} \cdot \hat{Z}_{n-1} & \hat{Z}_{n} \cdot \hat{Z}_{n-1}
\end{array}\right]
$$

sendo seus componentes referidos como cossenos diretores ${ }^{1}$.

Para os cálculos das matrizes de rotação a seguir, uma análise dos ângulos $\theta_{n}$ e $\psi_{n}$ é realizada pelas Figuras 19b a 22b, nas quais considera-se a planta orientada inicialmente na "configuração de reset" (definido na Seção 2.2). Fixa-se, então, o sistema de coordenadas $\{n-1\}$ e rotaciona-se $\{n\}$ em sentido positivo pela regra da mão direita até que o versor $\hat{X}_{n}$ forme um ângulo $\theta_{n}$ positivo com o versor $\hat{X}_{n-1}$. O ângulo $\psi_{n}$ indica o deslocamento angular resultante dos corpos. Nessas figuras são utilizados, como referência, os pontos de visão $n$ posicionados sobre os versores $\hat{Z}_{n}$, definidos nas Figuras 19a a 22a. Dessa forma, o sistema de coordenadas $\{n\}$ é rotacionado em sentido anti-horário nas Figuras 19b a $22 \mathrm{~b}$.

Feito isso, as matrizes de rotação ${ }_{n}^{n-1} R$ são calculadas e, como os sistemas de coordenadas $\{n\}$ são ortogonais, pode-se utilizar a propriedade ${ }_{n}^{n-1} R^{T}={ }_{n-1}^{n} R$, demonstrada na Seção 2.2 em Craig (2005), para calcular as matrizes de rotação ${ }_{n-1}^{n} R$, que são utilizadas para o cálculo dos vetores de velocidade angular ${ }^{n} \omega_{n}$ no Apêndice B.

\footnotetext{
${ }^{1}$ pois o produto escalar de dois vetores unitários fornece o cosseno do ângulo formado entre eles.
} 


\section{C.1 Cálculo da Matriz de Rotação $\left({ }_{0}^{1} R\right)$}

Para $n=1$, utiliza-se como referência o ponto de visão 1 da Figura 19a, fixa-se o sistema de coordenadas $\{0\}$ e rotaciona-se $\{1\}$ em sentido anti-horário em torno do eixo $\hat{Z}_{1}$. Os ângulos $\theta_{1}$ e $\psi_{1}$ podem ser analisados na Figura 19b.

Figura 19: Análise dos ângulos $\theta_{1}$ e $\psi_{1}$ para o cálculo de ${ }_{0}^{1} R$

(a) Ponto de Visão 1

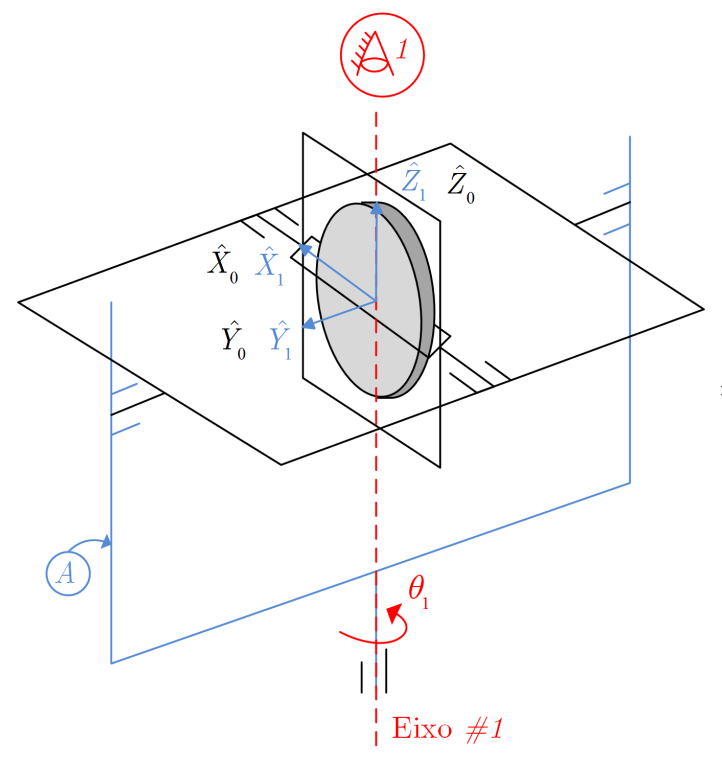

(b) Sistemas de Coordenadas $\{0\}$ e $\{1\}$ e deslocamento dos ângulos $\theta_{1}$ e $\psi_{1}$

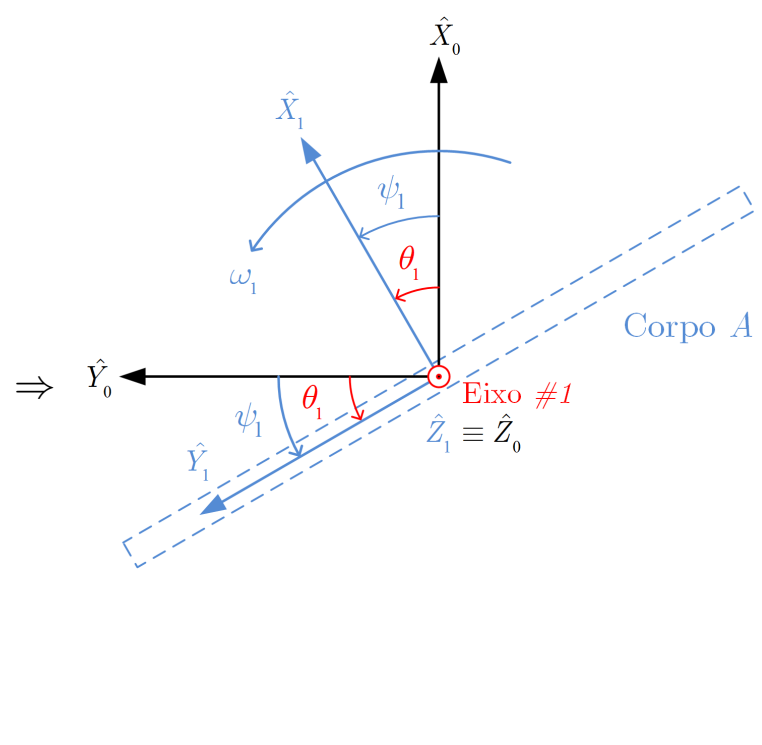

Fonte: Autor.

Substituindo $n=1$ em (C.1) obtém-se a matriz de rotação ${ }_{1}^{0} R$ :

$$
\begin{gathered}
{ }_{1}^{0} R=\left[\begin{array}{ccc}
\hat{X}_{1} \cdot \hat{X}_{0} & \hat{Y}_{1} \cdot \hat{X}_{0} & \hat{Z}_{1} \cdot \hat{X}_{0} \\
\hat{X}_{1} \cdot \hat{Y}_{0} & \hat{Y}_{1} \cdot \hat{Y}_{0} & \hat{Z}_{1} \cdot \hat{Y}_{0} \\
\hat{X}_{1} \cdot \hat{Z}_{0} & \hat{Y}_{1} \cdot \hat{Z}_{0} & \hat{Z}_{1} \cdot \hat{Z}_{0}
\end{array}\right]=\left[\begin{array}{ccc}
\cos \left(\theta_{1}\right) & \cos \left(\theta_{1}+90^{\circ}\right) & \cos \left(90^{\circ}\right) \\
\cos \left(90^{\circ}-\theta_{1}\right) & \cos \left(\theta_{1}\right) & \cos \left(90^{\circ}\right) \\
\cos \left(90^{\circ}\right) & \cos \left(90^{\circ}\right) & \cos \left(0^{\circ}\right)
\end{array}\right] \Rightarrow \\
{ }_{1}^{0} R=\left[\begin{array}{ccc}
\cos \left(\theta_{1}\right) & -\sin \left(\theta_{1}\right) & 0 \\
\sin \left(\theta_{1}\right) & \cos \left(\theta_{1}\right) & 0 \\
0 & 0 & 1
\end{array}\right],
\end{gathered}
$$

e como $\psi_{1}=\theta_{1}$, têm-se que:

$$
{ }_{1}^{0} R=\left[\begin{array}{ccc}
\cos \left(\psi_{1}\right) & -\sin \left(\psi_{1}\right) & 0 \\
\sin \left(\psi_{1}\right) & \cos \left(\psi_{1}\right) & 0 \\
0 & 0 & 1
\end{array}\right] .
$$


Por fim, utilizando a propriedade ${ }_{n-1}^{n} R={ }_{n}^{n-1} R^{T}$, para $n=1$, obtém-se:

$$
{ }_{0}^{1} R={ }_{1}^{0} R^{T}=\left[\begin{array}{ccc}
\cos \left(\psi_{1}\right) & -\sin \left(\psi_{1}\right) & 0 \\
\sin \left(\psi_{1}\right) & \cos \left(\psi_{1}\right) & 0 \\
0 & 0 & 1
\end{array}\right]^{T}=\left[\begin{array}{ccc}
\cos \left(\psi_{1}\right) & \sin \left(\psi_{1}\right) & 0 \\
-\sin \left(\psi_{1}\right) & \cos \left(\psi_{1}\right) & 0 \\
0 & 0 & 1
\end{array}\right]
$$

\section{C.2 Cálculo da Matriz de Rotação $\left({ }_{1}^{2} R\right)$}

De forma semelhante, para $n=2$ o ponto de visão 2 da Figura 20a é utilizado como referência. Fixa-se então o sistema de coordenadas $\{1\}$ e rotaciona-se $\{2\}$ em sentido anti-horário, em torno do eixo $\hat{Z}_{2}$, até que o ângulo $\theta_{2}$, formado entre os versores $\hat{X}_{2}$ e $\hat{X}_{1}$, seja positivo, o que resulta em $\psi_{2}=\theta_{2}+90^{\circ}$, como pode ser visto na Figura $20 \mathrm{~b}$.

Figura 20: Análise dos ângulos $\theta_{2}$ e $\psi_{2}$ para o cálculo de ${ }_{1}^{2} R$

(a) Ponto de Visão 2

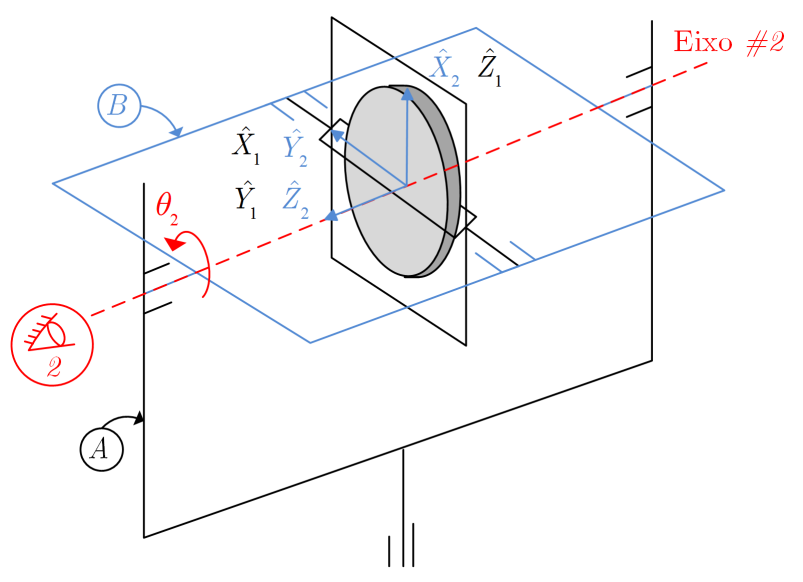

(b) Sistemas de Coordenadas $\{1\}$ e $\{2\}$ e deslocamento dos ângulos $\theta_{2}$ e $\psi_{2}$

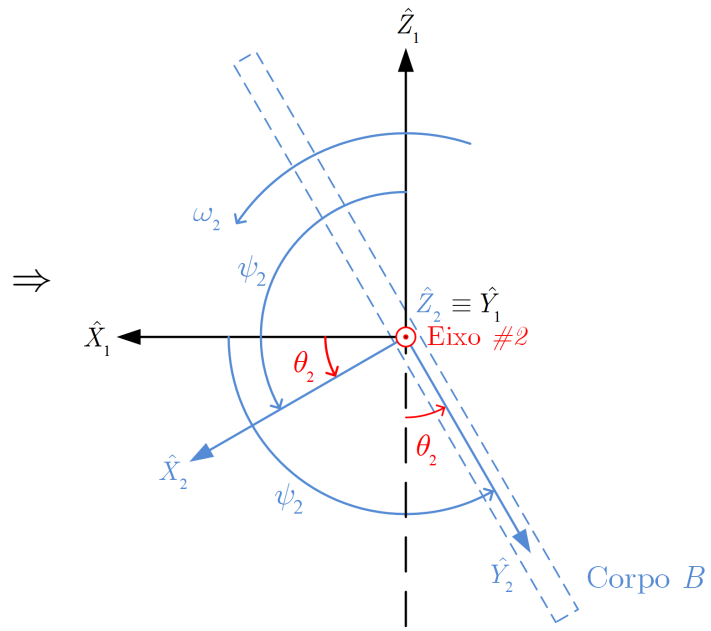

Fonte: Autor.

Substituindo $n=2$ em (C.1) obtém-se a matriz de rotação ${ }_{2}^{1} R$ :

$$
\begin{gathered}
{ }_{2}^{1} R=\left[\begin{array}{ccc}
\hat{X}_{2} \cdot \hat{X}_{1} & \hat{Y}_{2} \cdot \hat{X}_{1} & \hat{Z}_{2} \cdot \hat{X}_{1} \\
\hat{X}_{2} \cdot \hat{Y}_{1} & \hat{Y}_{2} \cdot \hat{Y}_{1} & \hat{Z}_{2} \cdot \hat{Y}_{1} \\
\hat{X}_{2} \cdot \hat{Z}_{1} & \hat{Y}_{2} \cdot \hat{Z}_{1} & \hat{Z}_{2} \cdot \hat{Z}_{1}
\end{array}\right]=\left[\begin{array}{ccc}
\cos \left(\theta_{2}\right) & \cos \left(\theta_{2}+90^{\circ}\right) & \cos \left(90^{\circ}\right) \\
\cos \left(90^{\circ}\right) & \cos \left(90^{\circ}\right) & \cos \left(0^{\circ}\right) \\
\cos \left(\theta_{2}+90^{\circ}\right) & \cos \left(\theta_{2}+180^{\circ}\right) & \cos \left(90^{\circ}\right)
\end{array}\right] \Rightarrow \\
{ }_{2}^{1} R=\left[\begin{array}{ccc}
\cos \left(\theta_{2}\right) & -\sin \left(\theta_{2}\right) & 0 \\
0 & 0 & 1 \\
-\sin \left(\theta_{2}\right) & -\cos \left(\theta_{2}\right) & 0
\end{array}\right],
\end{gathered}
$$


e como $\psi_{2}=\theta_{2}+90^{\circ} \Rightarrow \theta_{2}=\psi_{2}-90^{\circ}$, tem-se que:

$$
\begin{aligned}
\sin (A-B) & =\sin (A) \cos (B)-\cos (A) \sin (B) \Rightarrow \\
\sin \left(\theta_{2}\right)=\sin \left(\psi_{2}-90^{\circ}\right) & =\sin \left(\psi_{2}\right) \cos \left(90^{\circ}\right)-\cos \left(\psi_{2}\right) \sin \left(90^{\circ}\right)=-\cos \left(\psi_{2}\right), \\
\cos (A-B) & =\cos (A) \cos (B)+\sin (A) \sin (B) \Rightarrow \\
\cos \left(\theta_{2}\right)=\cos \left(\psi_{2}-90^{\circ}\right) & =\cos \left(\psi_{2}\right) \cos \left(90^{\circ}\right)+\sin \left(\psi_{2}\right) \sin \left(90^{\circ}\right)=\sin \left(\psi_{2}\right),
\end{aligned}
$$

e portanto, substituindo (C.4) e (C.5) em (C.3) obtém-se:

$$
{ }_{2}^{1} R=\left[\begin{array}{ccc}
\sin \left(\psi_{2}\right) & \cos \left(\psi_{2}\right) & 0 \\
0 & 0 & 1 \\
\cos \left(\psi_{2}\right) & -\sin \left(\psi_{2}\right) & 0
\end{array}\right]
$$

Por fim, como ${ }_{1}^{2} R={ }_{2}^{1} R^{T}$, têm-se que:

$$
{ }_{1}^{2} R=\left[\begin{array}{ccc}
\sin \left(\psi_{2}\right) & \cos \left(\psi_{2}\right) & 0 \\
0 & 0 & 1 \\
\cos \left(\psi_{2}\right) & -\sin \left(\psi_{2}\right) & 0
\end{array}\right]^{T}=\left[\begin{array}{ccc}
\sin \left(\psi_{2}\right) & 0 & \cos \left(\psi_{2}\right) \\
\cos \left(\psi_{2}\right) & 0 & -\sin \left(\psi_{2}\right) \\
0 & 1 & 0
\end{array}\right]
$$

\section{C.3 Cálculo da Matriz de Rotação $\left({ }_{2}^{3} R\right)$}

Analogamente, utilizando como referência o ponto de visão 3 da Figura 21a, fixa-se o sistema de coordenadas $\{2\}$ e rotaciona-se $\{3\}$ em sentido anti-horário em torno do eixo $\hat{Z}_{3}$ para a análise dos ângulos $\theta_{3}$ e $\psi_{3}$ na Figura 21 b.

Substituindo $n=3$ em (C.1) obtém-se a matriz de rotação ${ }_{3}^{2} R$ :

$$
\begin{gathered}
{ }_{3}^{2} R=\left[\begin{array}{ccc}
\hat{X}_{3} \cdot \hat{X}_{2} & \hat{Y}_{3} \cdot \hat{X}_{2} & \hat{Z}_{3} \cdot \hat{X}_{2} \\
\hat{X}_{3} \cdot \hat{Y}_{2} & \hat{Y}_{3} \cdot \hat{Y}_{2} & \hat{Z}_{3} \cdot \hat{Y}_{2} \\
\hat{X}_{3} \cdot \hat{Z}_{2} & \hat{Y}_{3} \cdot \hat{Z}_{2} & \hat{Z}_{3} \cdot \hat{Z}_{2}
\end{array}\right]=\left[\begin{array}{ccc}
\cos \left(\theta_{3}\right) & \cos \left(\theta_{3}+90^{\circ}\right) & \cos \left(90^{\circ}\right) \\
\cos \left(90^{\circ}\right) & \cos \left(90^{\circ}\right) & \cos \left(0^{\circ}\right) \\
\cos \left(\theta_{3}+90^{\circ}\right) & \cos \left(\theta_{3}+180^{\circ}\right) & \cos \left(90^{\circ}\right)
\end{array}\right] \Rightarrow \\
{ }_{3}^{2} R=\left[\begin{array}{ccc}
\cos \left(\theta_{3}\right) & -\sin \left(\theta_{3}\right) & 0 \\
0 & 0 & 1 \\
-\sin \left(\theta_{3}\right) & -\cos \left(\theta_{3}\right) & 0
\end{array}\right],
\end{gathered}
$$

e como $\psi_{3}=\theta_{3}$, tem-se que:

$$
{ }_{3}^{2} R=\left[\begin{array}{ccc}
\cos \left(\psi_{3}\right) & -\sin \left(\psi_{3}\right) & 0 \\
0 & 0 & 1 \\
-\sin \left(\psi_{3}\right) & -\cos \left(\psi_{3}\right) & 0
\end{array}\right]
$$


Figura 21: Análise dos ângulos $\theta_{3}$ e $\psi_{3}$ para o cálculo de ${ }_{2}^{3} R$

(a) Ponto de Visão 3

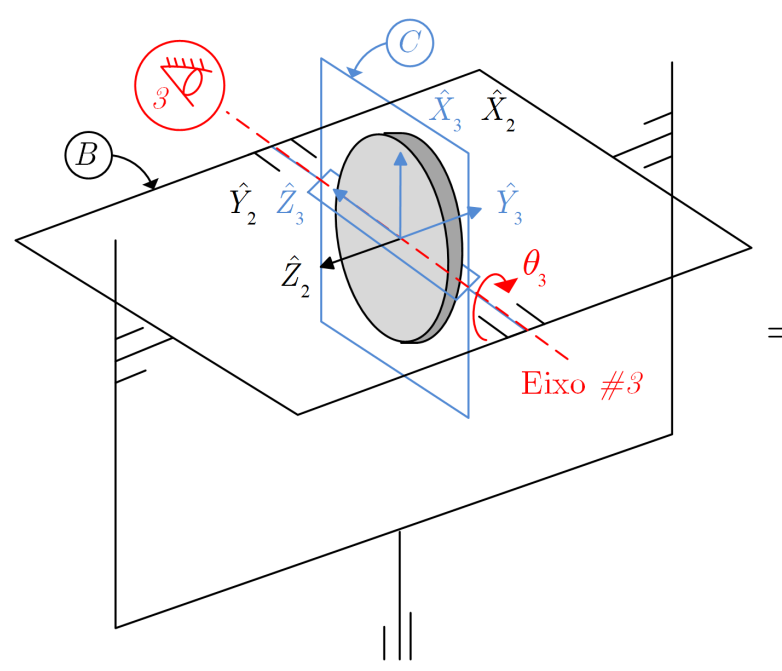

(b) Sistemas de Coordenadas $\{2\}$ e $\{3\}$ e deslocamento dos ângulos $\theta_{3}$ e $\psi_{3}$

Fonte: Autor.

Por fim, como ${ }_{2}^{3} R={ }_{3}^{2} R^{T}$, obtém-se:

$$
{ }_{2}^{3} R=\left[\begin{array}{ccc}
\cos \left(\psi_{3}\right) & -\sin \left(\psi_{3}\right) & 0 \\
0 & 0 & 1 \\
-\sin \left(\psi_{3}\right) & -\cos \left(\psi_{3}\right) & 0
\end{array}\right]^{T}=\left[\begin{array}{ccc}
\cos \left(\psi_{3}\right) & 0 & -\sin \left(\psi_{3}\right) \\
-\sin \left(\psi_{3}\right) & 0 & -\cos \left(\psi_{3}\right) \\
0 & 1 & 0
\end{array}\right] .
$$

\section{C.4 Cálculo da Matriz de Rotação $\left({ }_{3}^{4} R\right)$}

E por fim, utilizando o ponto de visão 4 da Figura 22a como referência, é fixado o sistema de coordenadas $\{3\}$ e rotacionado $\{4\}$ em sentido anti-horário em torno do eixo $\hat{Z}_{4}$, possibilitando a análise dos ângulos $\theta_{4}$ e $\psi_{4}$ na Figura $22 \mathrm{~b}$.

Substituindo $n=4$ em (C.1) obtém-se a matriz de rotação ${ }_{4}^{3} R$ :

$$
\begin{gathered}
{ }_{4}^{3} R=\left[\begin{array}{ccc}
\hat{X}_{4} \cdot \hat{X}_{3} & \hat{Y}_{4} \cdot \hat{X}_{3} & \hat{Z}_{4} \cdot \hat{X}_{3} \\
\hat{X}_{4} \cdot \hat{Y}_{3} & \hat{Y}_{4} \cdot \hat{Y}_{3} & \hat{Z}_{4} \cdot \hat{Y}_{3} \\
\hat{X}_{4} \cdot \hat{Z}_{3} & \hat{Y}_{4} \cdot \hat{Z}_{3} & \hat{Z}_{4} \cdot \hat{Z}_{3}
\end{array}\right]=\left[\begin{array}{ccc}
\cos \left(\theta_{4}\right) & \cos \left(\theta_{4}+90^{\circ}\right) & \cos \left(90^{\circ}\right) \\
\cos \left(90^{\circ}\right) & \cos \left(90^{\circ}\right) & \cos \left(180^{\circ}\right) \\
\cos \left(90^{\circ}-\theta_{4}\right) & \cos \left(\theta_{4}\right) & \cos \left(90^{\circ}\right)
\end{array}\right] \Rightarrow \\
{ }_{4}^{3} R=\left[\begin{array}{ccc}
\cos \left(\theta_{4}\right) & -\sin \left(\theta_{4}\right) & 0 \\
0 & 0 & -1 \\
\sin \left(\theta_{4}\right) & \cos \left(\theta_{4}\right) & 0
\end{array}\right],
\end{gathered}
$$


Figura 22: Análise dos ângulos $\theta_{4}$ e $\psi_{4}$ para o cálculo de ${ }_{3}^{4} R$

(a) Ponto de Visão 4

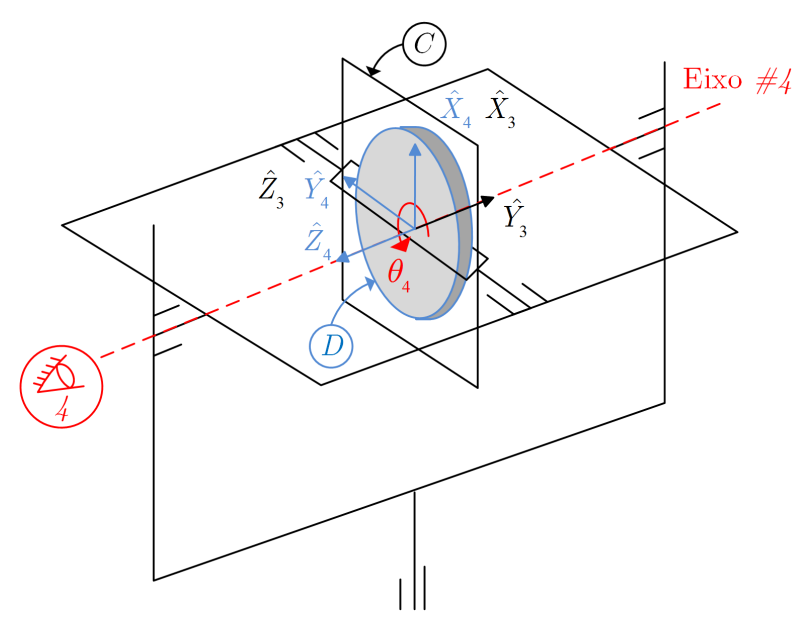

(b) Sistemas de Coordenadas $\{3\}$ e $\{4\}$ e deslocamento dos ângulos $\theta_{4}$ e $\psi_{4}$

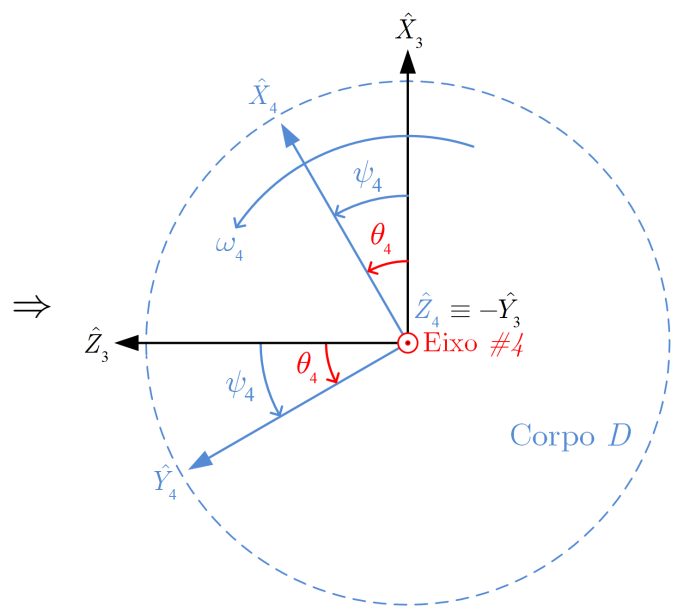

Fonte: Autor.

e como $\psi_{4}=\theta_{4}$, têm-se que:

$$
{ }_{4}^{3} R=\left[\begin{array}{ccc}
\cos \left(\psi_{4}\right) & -\sin \left(\psi_{4}\right) & 0 \\
0 & 0 & -1 \\
\sin \left(\psi_{4}\right) & \cos \left(\psi_{4}\right) & 0
\end{array}\right]
$$

Por fim, como ${ }_{3}^{4} R={ }_{4}^{3} R^{T}$, obtém-se:

$$
{ }_{3}^{4} R=\left[\begin{array}{ccc}
\cos \left(\psi_{4}\right) & -\sin \left(\psi_{4}\right) & 0 \\
0 & 0 & -1 \\
\sin \left(\psi_{4}\right) & \cos \left(\psi_{4}\right) & 0
\end{array}\right]^{T}=\left[\begin{array}{ccc}
\cos \left(\psi_{4}\right) & 0 & \sin \left(\psi_{4}\right) \\
-\sin \left(\psi_{4}\right) & 0 & \cos \left(\psi_{4}\right) \\
0 & -1 & 0
\end{array}\right]
$$




\section{APÊNDICE D - MATRIZES DE INÉRCIA CENTRAL DOS CORPOS}

As matrizes de inércia central dos corpos $m(m=A, B, C, D)$ são definidas com a notação $I_{n}(n=1,2,3,4)$, respectivamente, e são dadas em relação ao sistema de coordenadas inercial $\{0\}$. Seus elementos, definidos com as notações $I_{x x_{m}}, I_{y y_{m}}$ e $I_{z z_{m}}{ }^{1}$, são os momentos de inércia escalares em relação à direção dos versores $\hat{X}_{0}, \hat{Y}_{0}$ e $\hat{Z}_{0}$, respectivamente, nos corpos $m$.

$$
\begin{gathered}
I_{1}=\left[\begin{array}{ccc}
I_{x x_{A}} & 0 & 0 \\
0 & I_{y y_{A}} & 0 \\
0 & 0 & I_{z z_{A}}
\end{array}\right], I_{2}=\left[\begin{array}{ccc}
I_{z z_{B}} & 0 & 0 \\
0 & I_{x x_{B}} & 0 \\
0 & 0 & I_{y y_{B}}
\end{array}\right], \\
I_{3}=\left[\begin{array}{ccc}
I_{z z_{C}} & 0 & 0 \\
0 & I_{y y_{C}} & 0 \\
0 & 0 & I_{x x_{C}}
\end{array}\right], I_{4}=\left[\begin{array}{ccc}
I_{z z_{D}} & 0 & 0 \\
0 & I_{x x_{D}} & 0 \\
0 & 0 & I_{y y_{D}}
\end{array}\right],
\end{gathered}
$$

sendo $I_{z z_{D}}=I_{x x_{D}}$ devido ao formato axissimétrico do disco (Corpo $\left.D\right)$.

\footnotetext{
${ }^{1} I_{x x_{m}}, I_{y y_{m}}$ e $I_{z z_{m}}$ são equivalentes às notações $I_{m}, J_{m}$ e $K_{m}$ do manual da planta $(\mathrm{ECP}, 1999)$, respectivamente.
} 


\section{APÊNDICE E - EQUAÇÕES NÃO-LINEARES}

$T_{m_{4}}-I_{y y_{D}} \dot{\omega}_{4}-I_{y y_{D}} \cos \left(\psi_{3}\right) \dot{\omega}_{2}-I_{y y_{D}} \sin \left(\psi_{3}\right) \cos \left(\psi_{2}\right) \dot{\omega}_{1}+I_{y y_{D}} \sin \left(\psi_{3}\right) \omega_{3} \omega_{2}-$ $I_{y y_{D}} \cos \left(\psi_{3}\right) \cos \left(\psi_{2}\right) \omega_{3} \omega_{1}+I_{y y_{D}} \sin \left(\psi_{3}\right) \sin \left(\psi_{2}\right) \omega_{2} \omega_{1}=0$

$$
\begin{aligned}
& T_{m_{3}}-\left(I_{x x_{C}}+I_{x x_{D}}\right) \dot{\omega}_{3}+\left(I_{x x_{C}}+I_{x x_{D}}\right) \sin \left(\psi_{2}\right) \dot{\omega}_{1}-I_{y y_{D}} \sin \left(\psi_{3}\right) \omega_{4} \omega_{2}+ \\
& I_{y y_{D}} \cos \left(\psi_{3}\right) \cos \left(\psi_{2}\right) \omega_{4} \omega_{1}+\left(I_{x x_{D}}+I_{z z_{C}}-I_{y y_{C}}-I_{y y_{D}}\right) \cos \left(\psi_{3}\right) \sin \left(\psi_{3}\right) \omega_{2}^{2}+ \\
& {\left[2\left(I_{x x_{D}}+I_{z z_{C}}-I_{y y_{C}}-I_{y y_{D}}\right) \sin ^{2}\left(\psi_{3}\right)+\left(I_{x x_{C}}+I_{y y_{C}}+I_{y y_{D}}-I_{z z_{C}}\right)\right] \cos \left(\psi_{2}\right) \omega_{2} \omega_{1}+} \\
& \left(I_{y y_{C}}+I_{y y_{D}}-I_{x x_{D}}-I_{z z_{C}}\right) \cos ^{2}\left(\psi_{2}\right) \cos \left(\psi_{3}\right) \sin \left(\psi_{3}\right) \omega_{1}^{2}=0 \\
& -I_{y y_{D}} \cos \left(\psi_{3}\right) \dot{\omega}_{4}+\left[\left(I_{y y_{C}}+I_{y y_{D}}-I_{z z_{C}}-I_{x x_{D}}\right) \sin ^{2}\left(\psi_{3}\right)-\left(I_{y y_{B}}+I_{y y_{C}}+I_{y y_{D}}\right)\right] \dot{\omega}_{2}+ \\
& \left(I_{z z_{C}}+I_{x x_{D}}-I_{y y_{C}}-I_{y y_{D}}\right) \cos \left(\psi_{2}\right) \cos \left(\psi_{3}\right) \sin \left(\psi_{3}\right) \dot{\omega}_{1}+I_{y y_{D}} \sin \left(\psi_{3}\right) \omega_{4} \omega_{3}- \\
& I_{y y_{D}} \sin \left(\psi_{3}\right) \sin \left(\psi_{2}\right) \omega_{4} \omega_{1}+2\left(I_{y y_{C}}+I_{y y_{D}}-I_{z z_{C}}-I_{x x_{D}}\right) \cos \left(\psi_{3}\right) \sin \left(\psi_{3}\right) \omega_{3} \omega_{2}+ \\
& {\left[2\left(I_{y y_{C}}+I_{y y_{D}}-I_{z z_{C}}-I_{x x_{D}}\right) \sin ^{2}\left(\psi_{3}\right)+\left(I_{z z_{C}}-I_{x x_{C}}-I_{y y_{C}}-I_{y y_{D}}\right)\right] \cos \left(\psi_{2}\right) \omega_{3} \omega_{1}+} \\
& {\left[\left(I_{z z_{C}}+I_{x x_{D}}-I_{y y_{C}}-I_{y y_{D}}\right) \sin ^{2}\left(\psi_{3}\right)+\left(I_{x x_{B}}+I_{x x_{C}}-I_{z z_{B}}-I_{z z_{C}}\right)\right] \cos \left(\psi_{2}\right) \sin \left(\psi_{2}\right) \omega_{1}^{2}=0}
\end{aligned}
$$

$-I_{y y_{D}} \sin \left(\psi_{3}\right) \cos \left(\psi_{2}\right) \dot{\omega}_{4}+\left(I_{x x_{C}}+I_{x x_{D}}\right) \sin \left(\psi_{2}\right) \dot{\omega}_{3}+$

$$
\begin{aligned}
& \left(I_{z z_{C}}+I_{x x_{D}}-I_{y y_{C}}-I_{y y_{D}}\right) \cos \left(\psi_{2}\right) \cos \left(\psi_{3}\right) \sin \left(\psi_{3}\right) \dot{\omega}_{2}-\left(I_{z z_{A}}+I_{z z_{B}}+I_{z z_{C}}+I_{x x_{D}}\right) \dot{\omega}_{1}+ \\
& \left(I_{z z_{B}}+I_{z z_{C}}-I_{x x_{B}}-I_{x x_{C}}\right) \sin ^{2}\left(\psi_{2}\right) \dot{\omega}_{1}+\left(I_{z z_{C}}+I_{x x_{D}}-I_{y y_{C}}-I_{y y_{D}}\right) \sin ^{2}\left(\psi_{3}\right) \cos ^{2}\left(\psi_{2}\right) \dot{\omega}_{1}-
\end{aligned}
$$

$I_{y y_{D}} \cos \left(\psi_{3}\right) \cos \left(\psi_{2}\right) \omega_{4} \omega_{3}+I_{y y_{D}} \sin \left(\psi_{3}\right) \sin \left(\psi_{2}\right) \omega_{4} \omega_{2}+$

$$
\begin{aligned}
& {\left[2\left(I_{y y_{C}}+I_{y y_{D}}-I_{z z_{C}}-I_{x x_{D}}\right) \sin ^{2}\left(\psi_{3}\right)+\left(I_{z z_{C}}+I_{x x_{C}}+2 I_{x x_{D}}-I_{y y_{C}}-I_{y y_{D}}\right)\right] \cos \left(\psi_{2}\right) \omega_{3} \omega_{2}+} \\
& 2\left(I_{z z_{C}}+I_{x x_{D}}-I_{y y_{C}}-I_{y y_{D}}\right) \cos ^{2}\left(\psi_{2}\right) \cos \left(\psi_{3}\right) \sin \left(\psi_{3}\right) \omega_{3} \omega_{1}+ \\
& 2\left[\left(I_{y y_{C}}+I_{y y_{D}}-I_{z z_{C}}-I_{x x_{D}}\right) \sin ^{2}\left(\psi_{3}\right)+\left(I_{z z_{B}}+I_{z z_{C}}-I_{x x_{B}}-I_{x x_{C}}\right)\right] \cos \left(\psi_{2}\right) \sin \left(\psi_{2}\right) \omega_{2} \omega_{1}+ \\
& \left(I_{y y_{C}}+I_{y y_{D}}-I_{z z_{C}}-I_{x x_{D}}\right) \sin \left(\psi_{2}\right) \cos \left(\psi_{3}\right) \sin \left(\psi_{3}\right) \omega_{2}^{2}=0
\end{aligned}
$$




\section{APÊNDICE F - MODELO NÃO-LINEAR}

O modelo não-linear é derivado das equações não-lineares, obtidas e descritas no Apêndice E, ao calcular as soluções de (E.1) a (E.4) explicitamente para as variáveis de aceleração angular $\dot{\omega}_{n}(n=1,2,3,4)$, considerando os valores numéricos dos momentos de inércia da Tabela 1. Efetuando os cálculos com o auxílio do programa MATLAB ${ }^{\circledR}$, o modelo não-linear resultante é composto pelas quatro equações abaixo:

$$
\dot{\omega}_{4}=\frac{\eta_{4}}{\beta}, \quad \dot{\omega}_{3}=\frac{\eta_{3}}{\beta}, \quad \dot{\omega}_{2}=\frac{\eta_{2}}{\beta} \quad \text { e } \quad \dot{\omega}_{1}=\frac{\eta_{1}}{\beta}
$$

sendo:

$$
\begin{aligned}
& \beta=6168 \cos ^{2}\left(\psi_{2}\right)-1247 \cos ^{2}\left(\psi_{3}\right)+11051 ; \\
& \eta_{4}=\left[361197,2238 \cos ^{2}\left(\psi_{2}\right)+169322,3443 \cos ^{2}\left(\psi_{3}\right)+404798,5348\right] T_{m_{4}}- \\
& 135263,1579 \cos \left(\psi_{2}\right) \sin \left(\psi_{2}\right) \sin \left(\psi_{3}\right) T_{m_{3}}+5869,5\left[\sin \left(\psi_{2}\right) \omega_{1}-\omega_{3}\right] \cos \left(\psi_{3}\right) \sin \left(\psi_{3}\right) \omega_{4}+ \\
& {\left[3273,3684 \cos ^{2}\left(\psi_{2}\right)-4622,5 \cos ^{2}\left(\psi_{3}\right)+11051\right] \sin \left(\psi_{3}\right) \omega_{3} \omega_{2}+} \\
& {\left[-3273,3684 \cos ^{2}\left(\psi_{2}\right)+4622,5 \cos ^{2}\left(\psi_{3}\right)-9825,5\right] \cos \left(\psi_{2}\right) \cos \left(\psi_{3}\right) \omega_{3} \omega_{1}+} \\
& {\left[-3273,3684 \cos ^{2}\left(\psi_{2}\right)+4622,5 \cos ^{2}\left(\psi_{3}\right)+11051\right] \sin \left(\psi_{2}\right) \sin \left(\psi_{3}\right) \omega_{2} \omega_{1}+} \\
& {\left[3273,3684 \cos ^{2}\left(\psi_{2}\right)-4622,5 \cos ^{2}\left(\psi_{3}\right)+9825,5\right] \cos \left(\psi_{2}\right) \cos \left(\psi_{3}\right) \sin \left(\psi_{2}\right) \omega_{1}^{2} ; } \\
& \eta_{3}= {\left[15263.1579 \cos ^{2}\left(\psi_{2}\right) \cos ^{2}\left(\psi_{3}\right)+91501.5480 \cos ^{2}\left(\psi_{2}\right)-61108.7461 \cos ^{2}\left(\psi_{3}\right)+541549.9226\right] T_{m_{4}}-} \\
& 135263.1579 \cos ^{2}\left(\psi_{2}\right) \sin \left(\psi_{2}\right) \sin \left(\psi_{3}\right) T_{m_{3}}-3276 \cos \left(\psi_{2}\right) \cos \left(\psi_{3}\right) \sin \left(\psi_{2}\right) \omega_{4} \omega_{3}+ \\
& {\left[-6190.6765 \cos ^{2}\left(\psi_{2}\right)-1251.5846 \sin ^{2}\left(\psi_{3}\right)-9840.0441\right] \sin \left(\psi_{3}\right) \omega_{4} \omega_{2}+} \\
& {\left[2914.6765 \cos ^{2}\left(\psi_{2}\right)-1251.5846 \cos ^{2}\left(\psi_{3}\right)+14367.6287\right] \cos \left(\psi_{2}\right) \cos \left(\psi_{3}\right) \omega_{4} \omega_{1}+} \\
& {\left[-2210.6316 \cos ^{2}\left(\psi_{3}\right)+2894.6316\right] \sin ^{2}\left(\psi_{2}\right) \cos \left(\psi_{2}\right) \omega_{3} \omega_{2}-} \\
& 2210.6316 \cos ^{2}\left(\psi_{2}\right) \cos \left(\psi_{3}\right) \sin \left(\psi_{2}\right) \sin _{3}\left(\psi_{3}\right) \omega_{3} \omega_{1}+ \\
& {\left[-4875.4412 \cos ^{2}\left(\psi_{2}\right)+985.6801 \cos ^{2}\left(\psi_{3}\right)-8735.1654\right] \cos \left(\psi_{3}\right) \sin \left(\psi_{3}\right) \omega_{2}^{2}+} \\
& {\left[-8148.8096 \cos ^{2}\left(\psi_{2}\right)+11757.2030\right] \cos ^{2}\left(\psi_{2}\right) \omega_{2} \omega_{1}+} \\
& {\left[7540.2508 \cos ^{2}\left(\psi_{2}\right)-1971.3603 \cos ^{2}\left(\psi_{3}\right)+19419.6426\right] \cos { }^{2}\left(\psi_{3}\right) \cos \left(\psi_{2}\right) \omega_{2} \omega_{1}+} \\
& {\left[2664.8096 \cos ^{2}\left(\psi_{2}\right)-985.6801 \cos ^{2}\left(\psi_{3}\right)+10945.7970\right] \cos { }^{2}\left(\psi_{2}\right) \cos \left(\psi_{3}\right) \sin \left(\psi_{3}\right) \omega_{1}^{2} ; }
\end{aligned}
$$




$$
\begin{aligned}
\eta_{2}= & -\left[135263.1579 \cos ^{2}\left(\psi_{2}\right)+215000\right] \cos \left(\psi_{3}\right) T_{m_{4}}+15263.1579 \cos \left(\psi_{2}\right) \cos \left(\psi_{3}\right) \sin \left(\psi_{2}\right) \sin \left(\psi_{3}\right) T_{m_{3}}+ \\
& {\left[3276 \sin ^{2}\left(\psi_{2}\right)-9145.5\right]\left[\sin \left(\psi_{2}\right) \omega_{1}-\omega_{3}\right] \sin \left(\psi_{3}\right) \omega_{4}+} \\
& {\left[2210.6316 \cos ^{2}\left(\psi_{2}\right)+3375.5\right] \sin \left(\psi_{3}\right) \cos \left(\psi_{3}\right) \omega_{3} \omega_{2}-} \\
& {\left[2210.6316 \cos ^{2}\left(\psi_{2}\right) \cos ^{2}\left(\psi_{3}\right)+684 \cos ^{2}\left(\psi_{2}\right)+3375.5 \cos ^{2}\left(\psi_{3}\right)+1225.5\right] \cos \left(\psi_{2}\right) \omega_{3} \omega_{1}-} \\
& {\left[2210.6316 \cos ^{2}\left(\psi_{2}\right)+5869.5\right] \cos \left(\psi_{3}\right) \sin \left(\psi_{2}\right) \sin \left(\psi_{3}\right) \omega_{2} \omega_{1}+} \\
& {\left.\left[2210.6316 \cos ^{2}\left(\psi_{2}\right) \cos ^{2}\left(\psi_{3}\right)-5484 \cos ^{2}\left(\psi_{2}\right)+4622.5 \cos ^{2}\left(\psi_{3}\right)-9825.5\right] \sin \left(\psi_{2}\right) \cos \left(\psi_{2}\right) \omega_{1}^{2} ; \quad \text { F.5 }\right) } \\
\eta_{1}= & -135263.1579 \cos \left(\psi_{2}\right) \sin \left(\psi_{3}\right) T_{m_{4}}-\left[15263.1579 \cos ^{2}\left(\psi_{3}\right)-135263.1579\right] \sin \left(\psi_{2}\right) T_{m_{3}}+ \\
& 3276\left[\sin \left(\psi_{2}\right) \omega_{1}-\omega_{3}\right] \cos \left(\psi_{2}\right) \cos \left(\psi_{3}\right) \omega_{4}+\left[-2210.6316 \cos ^{2}\left(\psi_{3}\right)+2894.6316\right] \cos \left(\psi_{2}\right) \omega_{3} \omega_{2}- \\
& 2210.6316 \cos ^{2}\left(\psi_{2}\right) \cos \left(\psi_{3}\right) \sin \left(\psi_{3}\right) \omega_{3} \omega_{1}+\left[2210.6316 \cos ^{2}\left(\psi_{3}\right)+9441.3684\right] \sin \left(\psi_{2}\right) \cos \left(\psi_{2}\right) \omega_{2} \omega_{1}+ \\
& 2210.6316 \cos ^{2}\left(\psi_{2}\right) \cos \left(\psi_{3}\right) \sin \left(\psi_{2}\right) \sin \left(\psi_{3}\right) \omega_{1}^{2} .
\end{aligned}
$$

A validação deste modelo não-linear é realizada para avaliar a sua utilização nas simulações desta dissertação.

\section{F.1 Validação do Modelo Não-Linear}

A validação do modelo não-linear, obtido nesta dissertação, é realizada comparandose os resultados obtidos da sua simulação com a dos experimentos práticos, com a planta real, dos procedimentos "Nutation: Frequency \& Mode Shapes" e "Precession", descritos na Seção 6.2 do manual da planta (ECP, 1999). Estes dois procedimentos avaliam as principais características da planta, nos quais são calculadas a frequência de nutação, no primeiro procedimento, e a taxa de precessão, no segundo procedimento.

A precessão, descrita de forma genérica, é a mudança da orientação do eixo de rotação de um corpo rotacionando a uma dada velocidade angular. Esse fenômeno é explicado de forma didática em Butikov (2006) com o intuito de demonstrar o seu comportamento físico, e, de forma complementar, alguns livros de introdução à mecânica, como Teodorescu (2008) e Schaub (2003), explicam esse fenômeno com equações e demonstrações matemáticas.

E a nutação, também descrita de forma genérica, é a vibração que ocorre no eixo de rotação quando o corpo está sob a ação da precessão. A frequência dessa vibração é proporcional à velocidade angular do corpo, ou seja, quanto mais rápido o corpo estiver rotacionando mais rápida será a vibração. Esse fenômeno também é explicado de forma didática em Butikov (2006). 


\section{F.1.1 Descrição e Resultados Obtidos dos Procedimentos}

No primeiro procedimento Nutation: Frequency 8 Mode Shapes, a planta é posicionada na "configuração de reset", definida na Seção 2.2, o freio do eixo \#2 é ativado e, em seguida, um controlador PI (proporcional-integral) simples, que eleva a velocidade do disco $\omega_{4}$ a $\Omega=400$ RPM e o mantém nessa velocidade, é ativado. Em $t=15$ segundos, é aplicada uma tensão no motor \#3 de aproximadamente 5 volts (16000 counts (unidades do conversor D/A)) com intervalo de duração de 50 milissegundos. Dessa forma, o gimbal interno (corpo $C$ ) apresenta uma reação conhecida como "nutação", na qual sua velocidade angular $\omega_{3}$ oscila em uma certa frequência, que é calculada medindo-se o período desta oscilação.

No segundo procedimento Precession, realiza-se os mesmo passos do primeiro procedimento, alterando apenas o valor da tensão aplicada no motor \#3 para aproximadamente 2 volts (6000 counts (unidades do conversor D/A)) e o intervalo de duração para 8 segundos. Dessa forma, o gimbal interno (corpo $C$ ) é deslocado de sua posição inicial, mudando a orientação do eixo de rotação do disco, o que gera um torque induzido pela precessão, conhecido como "torque giroscópico", que causa um deslocamento angular da base giratória (corpo $A$ ) à uma velocidade angular $\omega_{1}$ constante. Esse valor constante resultante de $\omega_{1}$ é conhecida como "taxa de precessão".

Os sinais obtidos, ao executar o primeiro procedimento, estão ilustrados na Figura 23, na qual são apresentadas as velocidades $\omega_{3}$ e $\omega_{1}$ obtidas na simulação (gráfico (a)) e no experimento (gráfico (b)).

Figura 23: Gráficos com os sinais obtidos no procedimento Nutation: Frequency $\&$ Mode Shapes, sendo (a) da simulação e (b) do experimento

(a) Velocidades $\omega_{3}$ e $\omega_{1} \mathrm{em} \mathrm{rad} / \mathrm{s}$

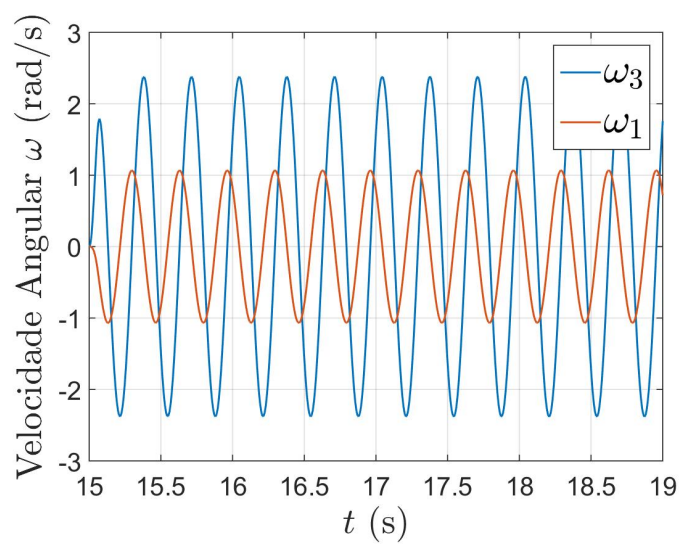

(b) Velocidades $\omega_{3}$ e $\omega_{1} \mathrm{em} \mathrm{rad} / \mathrm{s}$

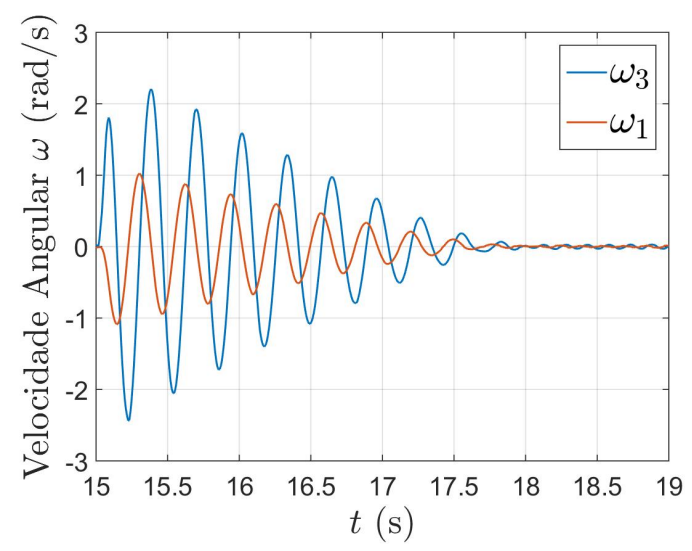

Fonte: Autor. 
Observa-se no gráfico (a) que a simulação apresentou uma resposta com velocidades oscilatórias sem amortecimento, fato já esperado pois não foram considerados os atritos nas juntas da planta durante a etapa de modelagem, como ressaltado no final da Seção 2.3; em contrapartida, no gráfico (b), observa-se que as velocidades obtidas no experimento prático foram amortecidas pelo atrito existente nas juntas da planta. Percebe-se que este fato não afeta a frequência de nutação na simulação, característica de interesse para avaliar o modelo não-linear obtido.

Portanto, para o cálculo da frequência de nutação, são coletados os intervalos de tempo entre o começo do $2^{\circ}$ ciclo $\left(t_{i}\right)$ e o fim do $4^{\circ}$ ciclo $\left(t_{f}\right)^{1}$ da oscilação de $\omega_{3}$, que na simulação são $t_{i}=15,30$ e $t_{f}=16,30$ segundos, e no experimento são $t_{i}=15,30$ e $t_{f}=16,26$ segundos. Calcula-se, então, o período da oscilação $T$ por meio da média da duração de um ciclo completo ${ }^{2}$ na simulação e no experimento, resultando em $T_{\text {simulação }} \approx 0,333$ segundos e $T_{\text {experimento }} \approx 0,320$ segundos, e suas respectivas frequências de nutação $f^{3}$, sendo $f_{\text {simulação }} \approx 18,868 \mathrm{rad} / \mathrm{s}$ e $f_{\text {experimento }} \approx 19,635 \mathrm{rad} / \mathrm{s}$.

Os sinais obtidos, ao executar o segundo procedimento, estão ilustrados na Figura 24, na qual são apresentadas as velocidades $\omega_{3}$ e $\omega_{1}$ obtidas na simulação (gráfico (a)) e no experimento (gráfico (b)), e os deslocamentos do gimbal interno $\psi_{3}$ e da base giratória $\psi_{1}$ obtidos na simulação (gráfico (c)) e no experimento (gráfico (d)). Observa-se que, de forma semelhante ao explicado no procedimento anterior, as respostas obtidas na simulação também não apresentaram amortecimento, fato que continua não afetando a característica de interesse, que neste procedimento é a taxa de precessão.

Como as velocidades obtidas nos gráficos das Figuras 24a e 24b apresentam oscilações, opta-se por calcular as velocidades $\omega_{1}$ por meio dos deslocamentos $\psi_{1}$, coletados nos gráficos das Figuras 24c e 24d, entre os instantes de tempo $t=17$ e $t=18$ segundos para obter valores mais precisos das taxas de precessão. Dessa forma, em $t=17$ segundos temse que $\psi_{1}=-0,9402 \mathrm{rad}$ na simulação e $\psi_{1}=-0,9972 \mathrm{rad}$ no experimento, e em $t=18$ segundos tem-se que $\psi_{1}=-1,4102 \mathrm{rad}$ na simulação e $\psi_{1}=-1,4806 \mathrm{rad}$ no experimento, que resultam nas taxas de precessão de $\omega_{1_{\text {simulacão }}}=-0,4700 \mathrm{rad} / \mathrm{s}$ e $\omega_{1_{\text {experimento }}}=-0,4834$ $\mathrm{rad} / \mathrm{s}$.

\footnotetext{
${ }^{1} \mathrm{O}$ primeiro ciclo é desconsiderado devido ao regime transitório da resposta, sendo optado a coleta do intervalo de tempo entre o começo do $2^{\circ}$ ciclo e o fim do $4^{\circ}$ ciclo, resultando em 3 ciclos completos $\left(n_{\text {ciclos }}=3\right)$.

${ }^{2}$ calculado pela relação $T=\frac{t_{f}-t_{i}}{n_{\text {ciclos }}}$, em segundos.

${ }^{3}$ calculado por $f=\frac{2 \pi}{T}$, em rad $/ \mathrm{s}$.
} 
Figura 24: Gráficos com os sinais obtidos no procedimento Precession, sendo [(a) e (c)] da simulação, e [(b) e (d)] do experimento

(a) Velocidades $\omega_{3}$ e $\omega_{1} \mathrm{em} \mathrm{rad} / \mathrm{s}$

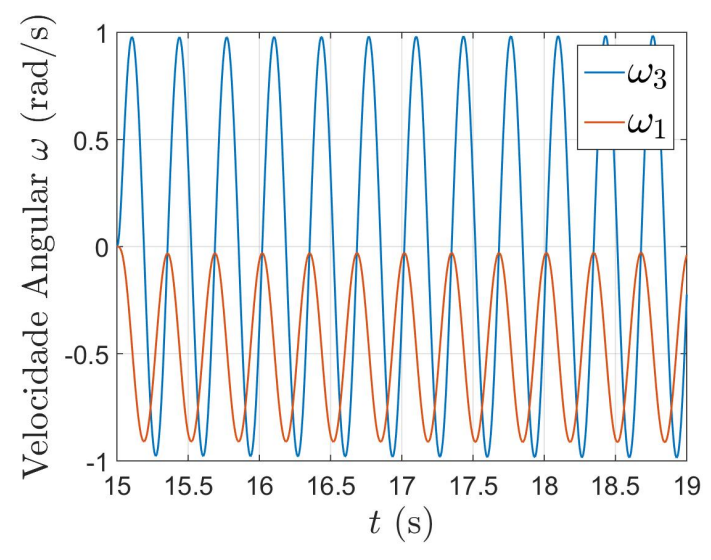

(c) Deslocamentos $\psi_{3}$ e $\psi_{1}$ em radianos

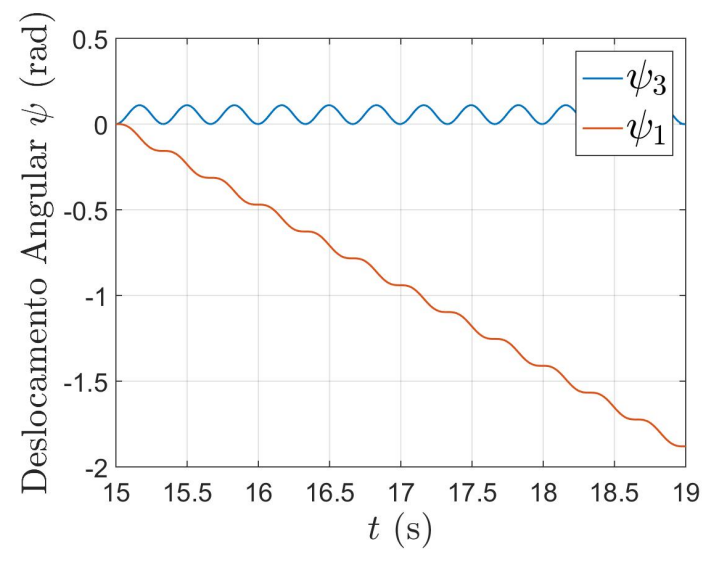

(b) Velocidades $\omega_{3}$ e $\omega_{1} \mathrm{em} \mathrm{rad} / \mathrm{s}$

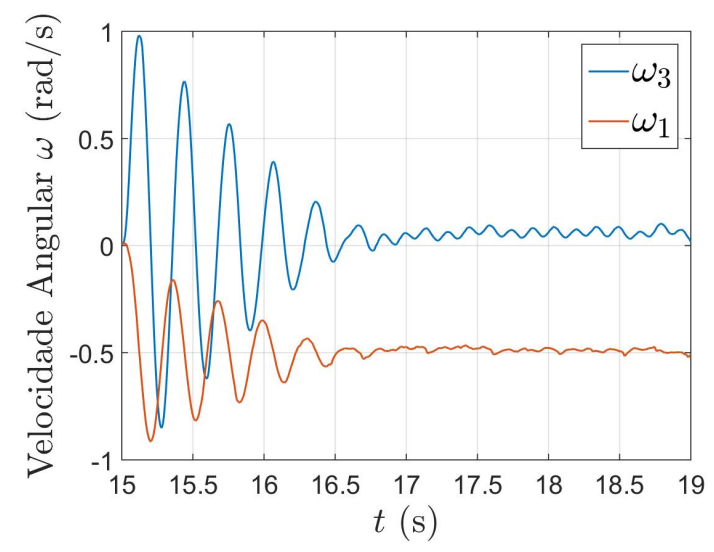

(d) Deslocamentos $\psi_{3}$ e $\psi_{1}$ em radianos

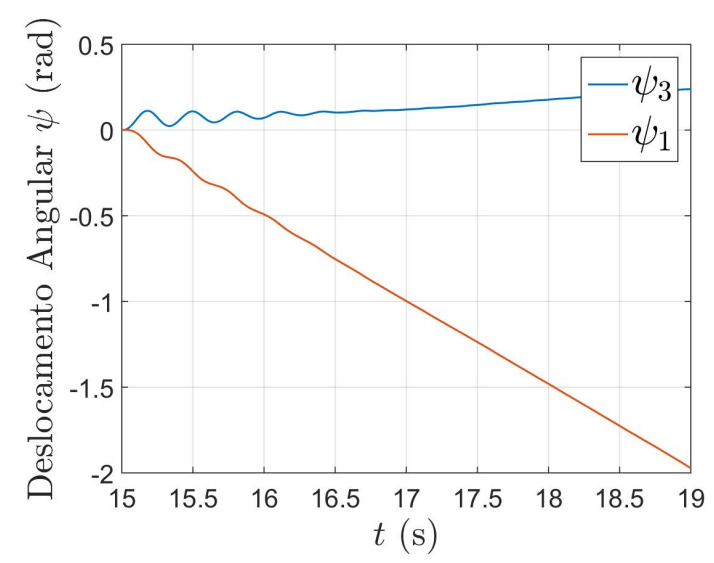

Fonte: Autor.

\section{F.1.2 Conclusão da Validação do Modelo Não-Linear}

Pode-se concluir que o modelo não-linear apresentou, de forma bem aproximada, as principais características da planta real, sendo que a frequência de nutação obtida na simulação apresentou um erro relativo percentual ${ }^{4}$, em relação à obtida no experimento, de $E_{\%}=3,91 \%$, e a taxa de precessão $E_{\%}=2,77 \%$.

Isso indica que, mesmo que não tenham sido consideradas algumas dinâmicas na etapa de modelagem da planta, como o atrito nas suas juntas, o modelo não-linear gerado é satisfatório para ser usado nas simulações desta dissertação, uma vez que os controladores são projetados com base em modelos lineares da planta.

$$
{ }^{4} E_{\%}=\left|\frac{x_{\text {simulação }}-x_{\text {experimento }}}{x_{\text {experimento }}}\right| \cdot 100 \%
$$




\section{APÊNDICE G - PARÂMETROS DA PLANTA}

Neste apêndice, são calculados os valores numéricos dos parâmetros inerentes à planta física, por meio de alguns procedimentos específicos fornecidos no manual da planta (ECP, 1999).

\section{G.1 Momentos de Inércia dos Corpos}

Os valores numéricos de uma parte dos momentos de inércia dos corpos são fornecidos pelo fabricante ${ }^{1}$ e estão relacionados na Tabela $5^{2}$.

Tabela 5: Momentos de inércia fornecidos pela ECP

\begin{tabular}{cccc}
\hline \multirow{2}{*}{ Corpo } & \multicolumn{2}{c}{ Momentos de Inércia } & \\
\cline { 2 - 3 } & \multicolumn{2}{c}{ Notações } & Valor $\left(\mathbf{K g} \cdot \mathbf{m}^{2}\right)$ \\
& do Manual & da Dissertação & \\
\hline $\mathrm{A}$ & $K_{A}$ & $I_{z z_{A}}$ & - \\
\hline \multirow{2}{*}{$\mathrm{B}$} & $I_{B}$ & $I_{x x_{B}}$ & 0,0119 \\
& $J_{B}$ & $I_{y y_{B}}$ & 0,0178 \\
& $K_{B}$ & $I_{z z_{B}}$ & 0,0297 \\
\hline \multirow{2}{*}{$\mathrm{C}$} & $I_{C}$ & $I_{x x_{C}}$ & - \\
& $J_{C}$ & $I_{y y_{C}}$ & - \\
\hline \multirow{2}{*}{$\mathrm{D}$} & $K_{C}$ & $I_{z z_{C}}$ & 0,0188 \\
& $I_{D}$ & $I_{x x_{D}}$ & 0,0148 \\
& $J_{D}$ & $I_{y y_{D}}$ & 0,0273 \\
\hline
\end{tabular}

Fonte: (ECP, 1999)

Os valores numéricos de $I_{y y_{C}}, I_{z z_{A}}$ e $I_{x x_{C}}{ }^{3}$ são calculados, respectivamente, com os dados obtidos ao executar ordenadamente os seguintes procedimentos: "Inertia Test

\footnotetext{
${ }^{1}$ mas poderiam também ser medidos experimentalmente usando o princípio da conservação de momento angular e outros princípios fundamentais, conforme informado no manual (ECP, 1999).

${ }^{2}$ As notações utilizadas no manual são diferentes das definidas nesta dissertação.

${ }^{3}$ De acordo com o manual, os valores destes momentos de inércia são os que mais variam de planta para planta, e são os que possuem a maior variância em relação ao valor obtido no cálculo pelas propriedades de massa (via geometria mecânica, densidade do material e pesos dos componentes).
} 
\#1 $\left(J_{C} \equiv I_{y y_{C}}\right)$ ", "Inertia Test \#2 $\left(K_{A} \equiv I_{z z_{A}}\right)$ " e "Inertia Test \#3 $\left(I_{C} \equiv I_{x x_{C}}\right)$ ", descritos na Subseção 6.1.1 do manual da planta (ECP, 1999).

No primeiro procedimento Inertia Test \#1 ( $\left.J_{C} \equiv I_{y y_{C}}\right)$, a planta é posicionada conforme Figura 25a, o freio do eixo \#1 é ativado e são aplicadas tensões $u_{m_{4}}$ no motor \#4 de aproximadamente 5 volts (16000 counts (unidades do conversor D/A)), durante o intervalo de tempo $t=1$ e $t=2$ segundos, e -5 volts $(-16000$ counts (unidades do conversor $\mathrm{D} / \mathrm{A})$ ), durante o intervalo de tempo $t=2$ e $t=3$ segundos. Dessa forma, o disco (corpo $D$ ) acelera (valor de $\omega_{4}$ aumenta positivamente) durante a aplicação da tensão com valor positivo e desacelera (valor de $\omega_{4}$ diminui) durante a aplicação da tensão com valor negativo; enquanto que o gimbal externo (corpo $B$ ) reage em sentido contrário ao disco, ou seja, ele acelera em sentido oposto (valor de $\omega_{2}$ aumenta negativamente) e desacelera durante a aplicação da tensão com valor negativo.

Figura 25: Configurações da planta para os procedimentos Inertia Tests
(a) Inertia Test \#1 $\left(I_{y y_{C}}\right)$
(b) Inertia Test \#2 $\left(I_{z z_{A}}\right)$
(c) Inertia Test \#3 $\left(I_{x x_{C}}\right)$
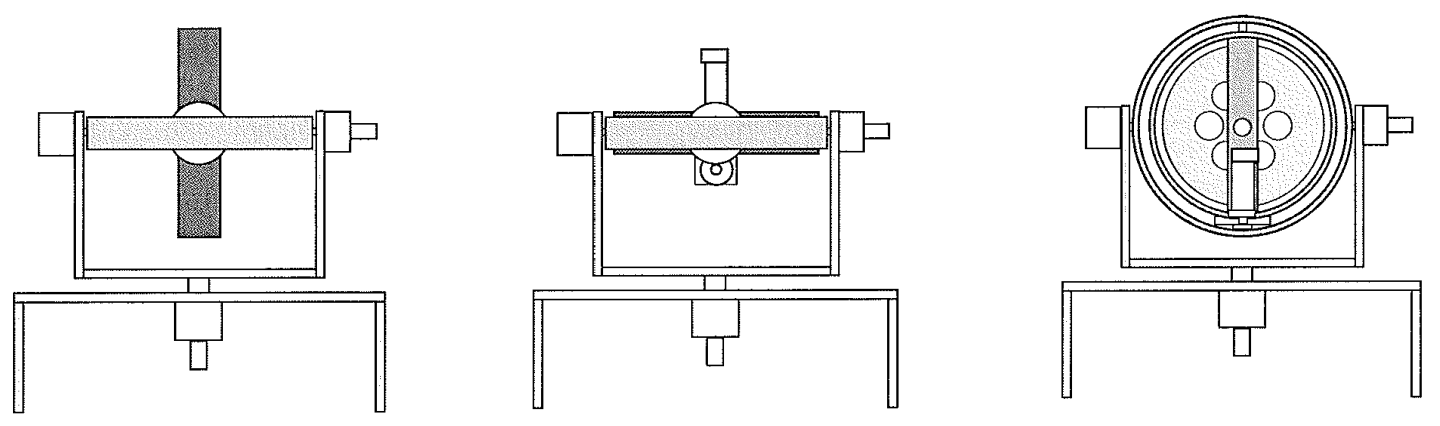

Fonte: Adaptado de (ECP, 1999).

No segundo procedimento Inertia Test \#2 $\left(K_{A} \equiv I_{z z_{A}}\right)$, a planta é posicionada conforme Figura 25b, o freio do eixo \#2 é ativado e são aplicadas tensões $u_{m_{4}}$ no motor \#4 de aproximadamente 5 volts (16000 counts (unidades do conversor D/A)), durante o intervalo de tempo $t=1$ e $t=3$ segundos, e -5 volts $(-16000$ counts (unidades do conversor $\mathrm{D} / \mathrm{A})$ ), durante o intervalo de tempo $t=3$ e $t=5$ segundos. Dessa forma, o disco (corpo $D$ ) se comporta da mesma maneira que no primeiro procedimento; porém agora a base giratória (corpo $A$ ) que reage em sentido contrário ao disco, ou seja, ela acelera em sentido oposto (valor de $\omega_{1}$ aumenta negativamente) e desacelera durante a aplicação da tensão com valor negativo.

E no terceiro procedimento Inertia Test \#3 $\left(I_{C} \equiv I_{x x_{C}}\right)$, a planta é posicionada conforme Figura 25c, o freio do eixo \#2 é ativado e são aplicadas tensões $u_{m_{3}}$ no motor \#3 de aproximadamente 5 volts (16000 counts (unidades do conversor D/A)), durante o 
intervalo de tempo $t=1$ e $t=1,2$ segundos, e -5 volts $(-16000$ counts (unidades do conversor D/A)), durante o intervalo de tempo $t=1,2$ e $t=1,4$ segundos. Dessa forma, o gimbal interno (corpo $C$ ) acelera (valor de $\omega_{3}$ aumenta positivamente) durante a aplicação da tensão com valor positivo e desacelera (valor de $\omega_{3}$ diminui) durante a aplicação da tensão com valor negativo; enquanto que a base giratória (corpo $A$ ) reage em sentido contrário ao gimbal interno, ou seja, ela acelera em sentido oposto (valor de $\omega_{1}$ aumenta negativamente) e desacelera durante a aplicação da tensão com valor negativo.

Os sinais obtidos, ao executar esses procedimentos, estão apresentados na Figura 26, na qual os gráficos (a), (b) e (c) exibem os sinais das velocidades, em rad/s, obtidos dos procedimentos Inertia Tests \#1 $\left(I_{y y_{C}}\right)$, \#2 $\left(I_{z z_{A}}\right)$ e \#3 $\left(I_{x x_{C}}\right)$, respectivamente.

Figura 26: Gráficos com os sinais das respostas obtidas nos procedimentos Inertia Tests: (a) \#1 $\left(I_{y y_{C}}\right)$, (b) \#2 $\left(I_{z z_{A}}\right)$ e $(\mathrm{c}) \# 3\left(I_{x x_{C}}\right)$
(a) $\omega_{4}$ e $\omega_{2} \mathrm{em} \mathrm{rad} / \mathrm{s}$
(b) $\omega_{4}$ e $\omega_{1} \mathrm{em} \mathrm{rad} / \mathrm{s}$
(c) $\omega_{3}$ e $\omega_{1} \mathrm{em} \mathrm{rad} / \mathrm{s}$
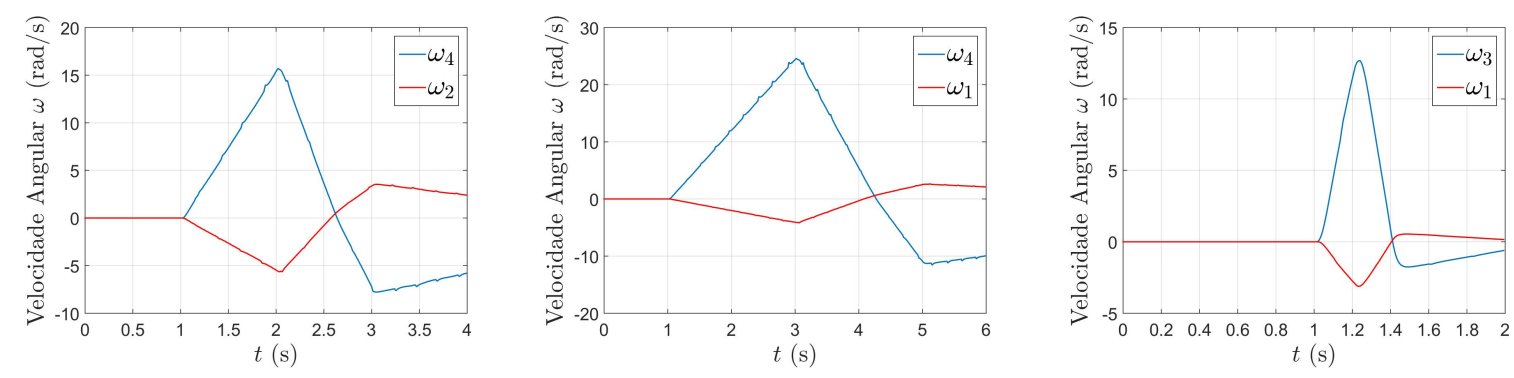

Fonte: Autor.

Por meio do princípio de conservação de momentos angulares de uma estrutura (os corpos da planta CMG, neste caso), que rotacionam em torno de um mesmo eixo sem a ação de um momento externo, tem-se a seguinte relação:

$$
J_{1} \Omega_{1}+J_{2} \Omega_{2}=C
$$

na qual são definidos os momentos de inércia e as velocidades angulares da estrutura $k$ $(k=1,2)^{4}$ com as notações $J_{k}$ e $\Omega_{k}{ }^{5}$, respectivamente, e $C$ é uma constante.

Igualando os momentos angulares dos instantes inicial e final, tem-se que:

$$
\begin{gathered}
J_{1} \Omega_{1_{o}}+J_{2} \Omega_{2_{o}}=J_{1} \Omega_{1_{f}}+J_{2} \Omega_{2_{f}} \Rightarrow \\
J_{1}=-J_{2} \cdot \frac{\left(\Omega_{2_{f}}-\Omega_{2_{o}}\right)}{\left(\Omega_{1_{f}}-\Omega_{1_{o}}\right)},
\end{gathered}
$$

\footnotetext{
${ }^{4}$ As estruturas 1 e 2 são compostas por um conjunto de corpos da planta e são diferentes entre si, conforme o procedimento a ser analisado.

${ }^{5}$ Vale ressaltar que a notação $\Omega_{k}$ é diferente da notação $\omega_{m}$, pois $\Omega_{k}$ é a velocidade angular total da estrutura $k(k=1,2)$ em relação ao sistema inercial $\{0\}$, ou seja, é a somatória das velocidades angulares dos corpos que compõe a estrutura $k$, e $\omega_{m}$ é a velocidade angular do corpo $m(m=A, B, C, D)$.
} 
sendo que $\Omega_{k_{o}}$ e $\Omega_{k_{f}}$ são, respectivamente, as velocidades inicial e final da estrutura $k$ em um determinado intervalo de tempo.

Na Tabela 6 estão relacionados os valores das velocidades inicial $\omega_{i_{o}}$ e final $\omega_{i_{f}}(i=$ 1,2,3,4), em radianos por segundo, coletados dos gráficos (a), (b) e (c) da Figura 26, sendo $\omega_{i_{o+}}$ e $\omega_{i_{f+}}$ os valores coletados do primeiro segmento da curva (intervalo de tempo da aplicação de tensão com valor positivo) e $\omega_{i_{o_{-}}}$e $\omega_{i_{f-}}$ do segundo segmento da curva (intervalo de tempo da aplicação de tensão com valor negativo).

Tabela 6: Velocidades obtidas nos procedimentos Inertial Tests

\begin{tabular}{ccccc}
\hline Inertial Test & $\omega_{i}(i=1,2,3,4)$ & $\omega_{i_{o+}}$ & $\omega_{i_{f+}}=\omega_{i_{o-}}$ & $\omega_{i_{f-}}$ \\
\hline \multirow{2}{*}{$\# 1\left(I_{y y_{C}}\right)$} & $\omega_{4}(i=4)$ & 0 & 15.69 & $-7,775$ \\
& $\omega_{2}(i=2)$ & 0 & $-5,637$ & 3,537 \\
\hline \multirow{2}{*}{$\left(I_{z z_{A}}\right)$} & $\omega_{4}(i=4)$ & 0 & 24,58 & $-11,29$ \\
& $\omega_{1}(i=1)$ & 0 & $-4,117$ & 2,583 \\
\hline \multirow{2}{*}{$\# 3\left(I_{x x_{C}}\right)$} & $\omega_{3}(i=3)$ & 0 & 12,69 & $-1,759$ \\
& $\omega_{1}(i=1)$ & 0 & $-3,118$ & 0,5469 \\
\hline
\end{tabular}

Fonte: Autor.

Com esses valores, são calculados os momentos de inércia $I_{y y_{C}}, I_{z z_{A}}$ e $I_{x x_{C}}$ aplicando a Equação (G.2) para o primeiro segmento (com $\omega_{i_{f+}}$ e $\omega_{i_{o+}}$ ) e para o segundo segmento $\left(\right.$ com $\omega_{i_{f-}}$ e $\left.\omega_{i_{o-}}\right)$ e realizando a média entre esses dois valores, ressaltando que:

- em Inertia Test \#1 $\left(I_{y y_{C}}\right): J_{1}=I_{y y_{B}}+I_{y y_{C}} ; J_{2}=I_{y y_{D}} ; \Omega_{1}=\omega_{2} ;$ e $\Omega_{2}=\omega_{4}+\omega_{2} ;$

- em Inertia Test \#2 $\left(I_{z z_{A}}\right): J_{1}=I_{z z_{A}}+I_{z z_{B}}+I_{y y_{C}} ; J_{2}=I_{y y_{D}} ; \Omega_{1}=\omega_{1} ;$ e $\Omega_{2}=\omega_{4}+\omega_{1}$;

- em Inertia Test \#3 $\left(I_{x x_{C}}\right): J_{1}=I_{x x_{C}}+I_{x x_{D}} ; J_{2}=I_{z z_{A}}+I_{x x_{B}} ; \Omega_{1}=\omega_{3}+\omega_{1} ; \mathrm{e}$ $\Omega_{2}=\omega_{1}$.

Os valores numéricos dos momentos de inércia $I_{z z_{A}}, I_{x x_{C}}$ e $I_{y y_{C}}$ resultantes estão relacionados na Tabela 7 .

Tabela 7: Momentos de inércia calculados

\begin{tabular}{cc}
\hline Momentos de Inércia & Valor $\mathbf{( K g . \mathbf { m } ^ { 2 } )}$ \\
\hline$I_{z z_{A}}$ & 0,0698 \\
$I_{x x_{C}}$ & 0,0124 \\
$I_{y y_{C}}$ & 0,0278 \\
\hline
\end{tabular}

Fonte: Autor. 


\section{G.2 Ganho dos Atuadores}

Outro parâmetro do giroscópio são os ganhos dos atuadores $k_{m_{h}}(h=4,3),{ }^{6}$ que relacionam os torques $T_{m_{h}}$ aplicados pelos motores $\# h$, em N.m, por unidades (counts) do conversor D/A (digital-analógico).

Esses ganhos teóricos, dados em N.m/counts (unidades do conversor D/A), são calculados através do produto:

$$
k_{m_{h}}=k_{c} \cdot k_{a} \cdot k_{t_{h}} \cdot k_{p_{h}}
$$

no qual:

- $k_{c}$ é o ganho do conversor D/A de 16-bit $=10 / 32768(\mathrm{~V} /$ counts $)$, sendo counts as unidades do conversor $\mathrm{D} / \mathrm{A}$;

- $k_{a}$ é o ganho do amplificador de corrente $\approx 0,4(\mathrm{~A} / \mathrm{V})$;

- $k_{t_{h}}$ é a constante de torque do motor $\approx 0,053$ (motor \#4), 0,12 (motor \#3) (N.m/A);

- $k_{p_{h}}$ é a relação de transmissão dos sistemas polia-correia $=3,33$ (motor \#4), 6,1 (motor \#3) (N.m saída/ N.m entrada).

E os ganhos experimentais, dados também em N.m/counts (unidades do conversor D/A), são calculados por meio das seguintes equações:

$$
\begin{gathered}
k_{m_{h}}=\frac{T_{m_{h}}}{u_{m_{h}}}, \\
T_{m_{4}}=I_{y y_{D}} \cdot \ddot{\psi}_{4}, \\
T_{m_{3}}=\omega_{1} \cdot H_{d i s c o}, \\
H_{d i s c o}=I_{x x_{D}} \cdot \Omega,
\end{gathered}
$$

sendo:

- $T_{m_{h}}$ o torque aplicado pelo motor \#h em N.m;

- $u_{m_{h}}$ o sinal de controle enviado para o motor \#h em counts (unidades do conversor $\mathrm{D} / \mathrm{A})$

\footnotetext{
${ }^{6}$ Esta constante envolve o ganho do conversor D/A (digital-analógico), o ganho estático do amplificador de corrente, a constante de torque do motor (a dinâmica do conjunto amplificador + motor é suficientemente mais rápida que a dinâmica do giroscópio e, portanto, é desprezada, sendo apenas o ganho considerado), e a relação de transmissão do sistema polia-correia.
} 
- $I_{y y_{D}}$ e $I_{x x_{D}}$ os momentos de inércia escalares do disco (corpo $D$ ), conforme Tabela 5

- $\ddot{\psi}_{4}$ a aceleração angular do disco em $\mathrm{rad} / \mathrm{s}^{2}$;

- $\omega_{1}$ a velocidade angular da base giratória (corpo $A$ ) em $\mathrm{rad} / \mathrm{s}$;

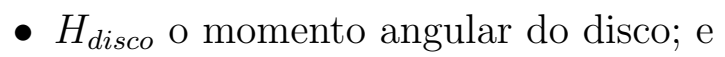

- $\Omega$ a velocidade angular constante do disco $\mathrm{em} \mathrm{rad} / \mathrm{s}$.

A equação (G.4) calcula o ganho $k_{m_{h}}$, relacionando o torque $T_{m_{h}}$ aplicado pelo motor $\#$, em N.m, com o sinal de controle $u_{m_{h}}$ enviado, em counts (unidades do conversor D/A). O torque aplicado $T_{m_{4}}$ pelo motor \#4 é calculado por meio da segunda Lei de Newton, em sua forma rotacional, que é representada pela equação (G.5), e o torque aplicado $T_{m_{3}}$ pelo motor \#3 é calculado por meio do produto vetorial giroscópico, que é representado pela equação (G.6), na qual o momento angular do disco $H_{\text {disco é calculado }}$ pela equação (G.7).

Os dados para o cálculo dos ganhos experimentais são obtidos ao executar os procedimentos "Control Effort Gain Test \#1 $\left(k_{g_{1}} \equiv k_{m_{4}}\right)$ " e "Control Effort Gain Test \#2 $\left(k_{g_{2}} \equiv k_{m_{3}}\right) " 7$, descritos na Subseção 6.1.2 do manual da planta (ECP, 1999).

No procedimento Control Effort Gain Test \#1 $\left(k_{g_{1}} \equiv k_{m_{4}}\right)$, a planta é posicionada conforme Figura 25c, os freios dos eixos \#1 e \#2 são ativados e são aplicadas tensões $u_{m_{4}}$ no motor \#4 de aproximadamente 5 volts (16000 counts (unidades do conversor D/A)), durante o intervalo de tempo $t=1$ e $t=5$ segundos, e -5 volts $(-16000$ counts (unidades do conversor $\mathrm{D} / \mathrm{A})$ ), durante o intervalo de tempo $t=5$ e $t=9$ segundos. Dessa forma, o disco (corpo $D$ ) acelera (valor de $\omega_{4}$ aumenta positivamente) durante a aplicação da tensão com valor positivo e desacelera (valor de $\omega_{4}$ diminui) durante a aplicação da tensão com valor negativo.

E no procedimento Control Effort Gain Test \#2 $\left(k_{g_{2}} \equiv k_{m_{3}}\right)$, a planta é posicionada conforme Figura 25a, o freio do eixo \#2 é ativado e, em seguida, dois controladores são ativados: um controlador PID (proporcional-integral-derivativo) simples que mantém o gimbal interno (corpo $C$ ) na posição vertical, e um controlador PI (proporcional-integral) simples que eleva a velocidade do disco $\omega_{4}$ a $\Omega=400$ RPM e o mantém nessa velocidade. Após alguns segundos, são aplicadas tensões $u_{m_{3}}$ no motor \#3 de aproximadamente 5

\footnotetext{
${ }^{7}$ Semelhante ao caso dos momentos de inércia, a notação definida aqui $\left(k_{m_{4}}\right.$ e $\left.k_{m_{3}}\right)$ é diferente do manual da planta $\left(k_{g_{1}}\right.$ e $k_{g_{2}}$, respectivamente).
} 
volts (16000 counts (unidades do conversor D/A)), durante o intervalo de tempo $t=15$ e $t=17$ segundos, e -5 volts $(-16000$ counts (unidades do conversor $\mathrm{D} / \mathrm{A})$ ), durante o intervalo de tempo $t=17$ e $t=19$ segundos. Dessa forma, como há um controle ativo para manter a posição vertical do gimbal interno (corpo $C$ ), a tensão aplicada $u_{m_{3}}$ é atenuada e varia entre aproximadamente 2,5 e 1,5 volts durante a aplicação da tensão com valor positivo, e varia entre -4 e -3 volts durante a aplicação da tensão com valor negativo; e, como o disco está rotacionando a uma velocidade constante de $\Omega=400$ RPM, um torque induzido pela precessão, chamado de "torque giroscópico", é gerado toda vez que uma tensão é aplicada no motor \#3, o que gera uma velocidade $\omega_{1}$ da base giratória (corpo $A$ ) em sentido oposto a tensão aplicada no motor \#3. O objetivo é medir o sinal de controle do motor \#3 $\left(u_{m_{3}}\right)$, em volts, durante um período em que a velocidade da base giratória $\omega_{1}$ esteja aproximadamente constante.

Executando esses procedimentos, obtêm-se os sinais apresentados na Figura 27, na qual os gráficos (a) e (b) exibem os sinais das velocidades, em rad/s, obtidos dos procedimentos Control Effort Gain Tests \#1 $\left(k_{m_{4}}\right)$ e \#2 $\left(k_{m_{3}}\right)$, respectivamente, e o gráfico (c) exibe o sinal resultante da tensão aplicada no motor \#3 $\left(u_{m_{3}}\right)$, em volts, obtido do procedimento Control Effort Gain Test \#2 $\left(k_{m_{3}}\right)$.

Figura 27: Gráficos com os sinais obtidos nos procedimentos Control Effort Gain Tests: (a) \#1 $\left(k_{m_{4}}\right)$, (b) e (c) \#2 $\left(k_{m_{3}}\right)$

(a) $\omega_{4} \mathrm{em} \mathrm{rad} / \mathrm{s}$

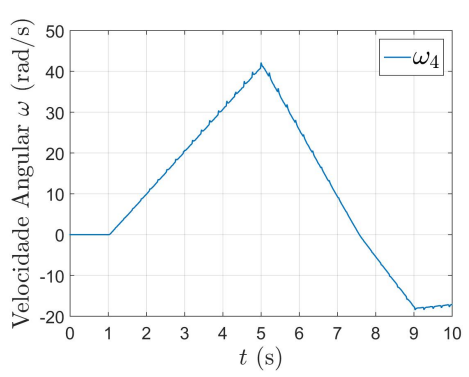

(b) $\omega_{1} \mathrm{em} \mathrm{rad} / \mathrm{s}$

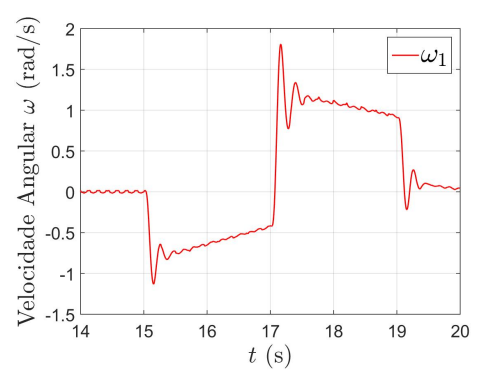

Fonte: Autor. (c) $u_{m_{3}}$ em volts

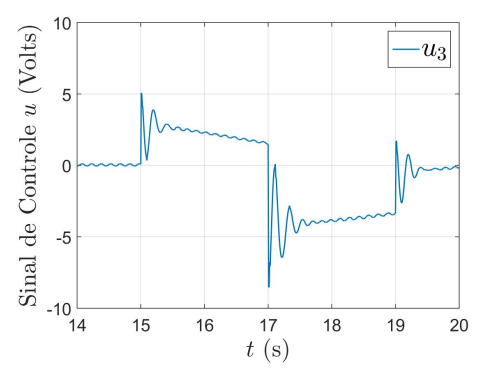

Para o cálculo de $k_{m_{4+}}$ e $k_{m_{4-}}$, são utilizadas as Equações (G.4) e (G.5), sendo a aceleração angular do disco $\ddot{\psi}_{4}$ obtida pela variação da velocidade $\omega_{4}$ no primeiro e no segundo segmento, causado pelo sinal de controle $u_{m_{4}}$ aplicado no procedimento Control Effort Gain Test \#1 $\left(k_{m_{4}}\right)$, sendo este convertido de volts para counts (unidades do conversor $\mathrm{D} / \mathrm{A}$ ) por meio da constante do ganho do conversor $\mathrm{D} / \mathrm{A}\left(k_{c}\right)$. Os valores da velocidade $\omega_{4}$, em radianos por segundo, são coletados do gráfico da Figura 27a, na qual $\omega_{4_{i+}}$ e $\omega_{4_{f+}}$ são coletados no primeiro segmento (intervalo de tempo da aplicação de tensão com valor positivo) e $\omega_{4_{i-}}$ e $\omega_{4_{f-}}$ no segundo segmento (intervalo de tempo da aplicação de 
tensão com valor negativo), sendo $\omega_{4_{i+}}$ nulo, $\omega_{4_{f+}}=\omega_{4_{i_{-}}}=42,16 \mathrm{rad} / \mathrm{s}$ e $\omega_{4_{f-}}=-18,45$ $\mathrm{rad} / \mathrm{s}$.

Para o cálculo de $k_{m_{3+}}$ e $k_{m_{3-}}$, são utilizadas as Equações (G.4), (G.6) e (G.7), sendo que $\Omega=400 \mathrm{RPM}=41,8879 \mathrm{rad} / \mathrm{s}$ no procedimento Control Effort Gain Test \#2 $\left(k_{m_{3}}\right)$, e os sinais da velocidade $\omega_{1}$, em radianos por segundos, são coletados dos gráficos da Figura $27 \mathrm{~b}$ e os sinais de controle $u_{m_{3}}$, em volts, da Figura $27 \mathrm{c}$, sendo $\omega_{1+}$ e $u_{m_{3+}}$ coletados no primeiro segmento (intervalo de tempo com sinal de controle positivo) e $\omega_{1-}$ e $u_{m_{3-}}$ no segundo segmento (intervalo de tempo com sinal de controle negativo). Esses valores estão relacionados na Tabela 8.

Tabela 8: Valores obtidos no procedimento Control Effort Gain Test \#2

\begin{tabular}{cccc}
\hline & tempo $(\mathbf{s})$ & $\omega_{1}(\mathrm{rad} / \mathbf{s})$ & $u_{m_{3}}$ (volts) \\
\hline \multirow{2}{*}{ Primeiro Segmento } & 15,54 & $\omega_{1_{i+}}=-0,7554$ & $u_{3_{i+}}=2,557$ \\
& 17 & $\omega_{1_{f+}}=-0,4156$ & $u_{3_{f+}}=1,473$ \\
\hline \multirow{2}{*}{ Segundo Segmento } & 17,66 & $\omega_{1_{i-}}=1,118$ & $u_{3_{i-}}=-4,09$ \\
& 19 & $\omega_{1_{f-}}=0,9098$ & $u_{3_{f-}}=-3,357$ \\
\hline
\end{tabular}

Fonte: Autor.

Efetuando a média entre os valores calculados para o primeiro e o segundo segmentos, têm-se os valores numéricos resultantes dos ganhos experimentais $k_{m_{4}}$ e $k_{m_{3}}$, relacionados na Tabela 9, na qual também estão relacionados os valores calculados dos ganhos teóricos.

Tabela 9: Ganhos dos atuadores calculados

\begin{tabular}{cc}
\hline Ganhos dos Atuadores & Valor (N.m/counts) \\
\hline$k_{m_{4}}$ teórico & $2.1544 \times 10^{-5}$ \\
$k_{m_{4}}$ experimental & $2.1919 \times 10^{-5}$ \\
\hline$k_{m_{3}}$ teórico & $8.9355 \times 10^{-5}$ \\
$k_{m_{3}}$ experimental & $9.8215 \times 10^{-5}$ \\
\hline
\end{tabular}

Fonte: Autor.

Nesta dissertação, optou-se pelo uso dos ganhos teóricos. 


\section{G.3 Ganho dos Encoders Incrementais}

Os ganhos $k_{e_{m}}$ dos encoders incrementais $m(m=A, B, C, D)$ relacionam a quantidade de pulsos de encoder (counts) por radianos de deslocamento angular dos corpos da planta CMG.

No procedimento "Feedback Sensor (Encoder) Gain Measurement", descrito na Subseção 6.1.3 do manual da planta (ECP, 1999), verifica-se a quantidade de pulsos de encoder (counts) no sinal de saída do encoder $m$ após efetuado uma revolução completa do corpo $m$. Com essa relação, pode-se calcular o ganho $k_{e_{m}}$, que relaciona a quantidade de pulsos de encoder (counts) por radianos.

Executando esses procedimentos, obtêm-se os valores numéricos relacionados na Tabela 10, sendo o fator multiplicativo "32" um ganho de firmware que aumenta a resolução interna dos encoders (de acordo com ECP (1999) pág. 72).

Tabela 10: Ganhos dos encoders calculados

\begin{tabular}{ccc}
\hline $\begin{array}{c}\text { Encoder } \\
m\end{array}$ & $\begin{array}{c}\text { Pulsos de encoder/Revolução } \\
\text { (counts } / \text { rev })\end{array}$ & $\begin{array}{c}\text { Ganho } k_{e_{m}} \\
\text { (counts } / \text { rad) }\end{array}$ \\
\hline$A$ & 16000 & $2547 \cdot 32$ \\
$B$ & 16000 & $2547 \cdot 32$ \\
$C$ & 24400 & $3883 \cdot 32$ \\
$D$ & 6667 & $1061 \cdot 32$ \\
\hline
\end{tabular}

Fonte: Autor.

Com isso, têm-se todos os valores numéricos dos parâmetros necessários para obter os modelos da planta. 


\section{APÊNDICE H - RESULTADOS DAS SIMULAÇÕES E EXPERIMENTOS}

Neste apêndice, são apresentados os resultados obtidos nas simulações e experimentos práticos dos testes realizados com os sistemas contendo os controladores digitais projetados e a planta nas três configurações exploradas nesta dissertação (caso desacoplado de fase mínima e casos acoplados de fase mínima e não-mínima). São apresentados nos gráficos da esquerda $[(a),(c)$ e (e)] os sinais obtidos na simulação e nos gráficos da direita [(b), (d) e (f)] os sinais obtidos no experimento prático. Os gráficos (a) e (b) exibem os sinais de saída do sistema $y(t)=\left[\psi_{2}(t) \psi_{1}(t)\right]^{T}{ }^{1}$ e os sinais de acompanhamento de referência $r(t)=\left[r_{\psi_{2}}(t) r_{\psi_{1}}(t)\right]^{T}$ ou os sinais de perturbação na saída da planta $d(t)=\left[\begin{array}{ll}d_{\psi_{2}} & d_{\psi_{1}}\end{array}\right]^{T} 2$, dependendo do teste realizado; os gráficos (c) e (d) exibem os sinais de controle $u(t)=\left[T_{m_{4}}(t) T_{m_{3}}(t)\right]^{T}{ }^{3}$ com os torques aplicados pelos motores \#4 e \#3, respectivamente, e o limite de saturação dos motores representadas pelas linhas pretas tracejadas; e os gráficos (e) e (f) exibem as velocidades angulares $\omega_{3}\left(k T_{s}\right), \omega_{2}\left(k T_{s}\right)$ e $\omega_{1}\left(k T_{s}\right)^{4}$, do vetor de estados da planta $x\left(k T_{s}\right)$, obtidas pelo método retangular para trás, dos corpos $C, B$ e $A$, respectivamente.

Os gráficos, neste apêndice, começam no instante de tempo $t=14$ segundos, para os ensaios com pulsos retangulares (ensaios 1 e 2), e $t=11$ segundos, para os ensaios com sinais senoidais (ensaio 3), pois na parte inicial do procedimento experimental são realizados apenas procedimentos que levam a planta ao seu ponto de operação e para ativar o controlador projetado. É a partir da inserção dos sinais dos ensaios que são coletados os dados de interesse dos resultados, com os quais é analisado o desempenho do sistema. A Figura 16, na Seção 6.1, apresenta um exemplo do procedimento experimental

\footnotetext{
${ }^{1} \psi_{2}(t)$ é representado pela curva verde contínua e $\psi_{1}(t)$ pela curva azul contínua.

${ }^{2} r_{\psi_{2}}(t)$ e $d_{\psi_{2}}(t)$ são representados pelas curvas vermelhas tracejadas e $r_{\psi_{1}}(t)$ e $d_{\psi_{1}}(t)$ pelas curvas laranjas tracejadas.

${ }^{3} T_{m_{4}}(t)$ é representado pela curva azul e $T_{m_{3}}(t)$ pela curva vermelha.

${ }^{4} \omega_{3}\left(k T_{s}\right)$ é representado pela curva lilás, $\omega_{2}\left(k T_{s}\right)$ pela curva verde e $\omega_{1}\left(k T_{s}\right)$ pela curva ciano.
} 
completo para o ensaio 3, na qual observa-se que não há dados de interesse para a análise do sistema até $t=11$ segundos.

\section{H.1 Caso Desacoplado}

Para o sistema desacoplado realiza-se o procedimento experimental, para cada ensaio de sinais considerados (Ensaios 1, 2 e 3), com os sistemas contendo a planta na configuração desacoplada (no ponto de operação $\psi_{2_{0}}=0^{\circ}, \psi_{3_{0}}=0^{\circ}$ e $\Omega=400 R P M$ ) e os controladores digitais projetados.

\section{H.1.1 Sistema com o Controlador PID}

Para o sistema desacoplado, o controlador digital PID utilizado é projetado em relação ao modelo desacoplado da planta. Os resultados obtidos para os 3 ensaios de sinais considerados são apresentados a seguir.

\section{H.1.1.1 Ensaio 1 - Pulsos Retangulares Separados}

As Figuras 28 e 29 apresentam os resultados obtidos no teste de acompanhamento de referência com amplitudes positivas e negativas, respectivamente. e as Figuras 30 e 31 os resultados obtidos no teste de rejeição à perturbação com amplitudes positivas e negativas, respectivamente. 
Figura 28: Gráficos com os resultados do teste de acompanhamento de referência com sinais do ensaio 1 (positivo) - Sistema desacoplado com controle PID, com os sinais obtidos na simulação [(a), (c) e (e)] e no experimento [(b), (d) e (f)]
(a) Sinais $r(t)$ e $y(t) \mathrm{em}^{\circ}$
(b) Sinais $r(t)$ e $y(t) \mathrm{em}^{\circ}$
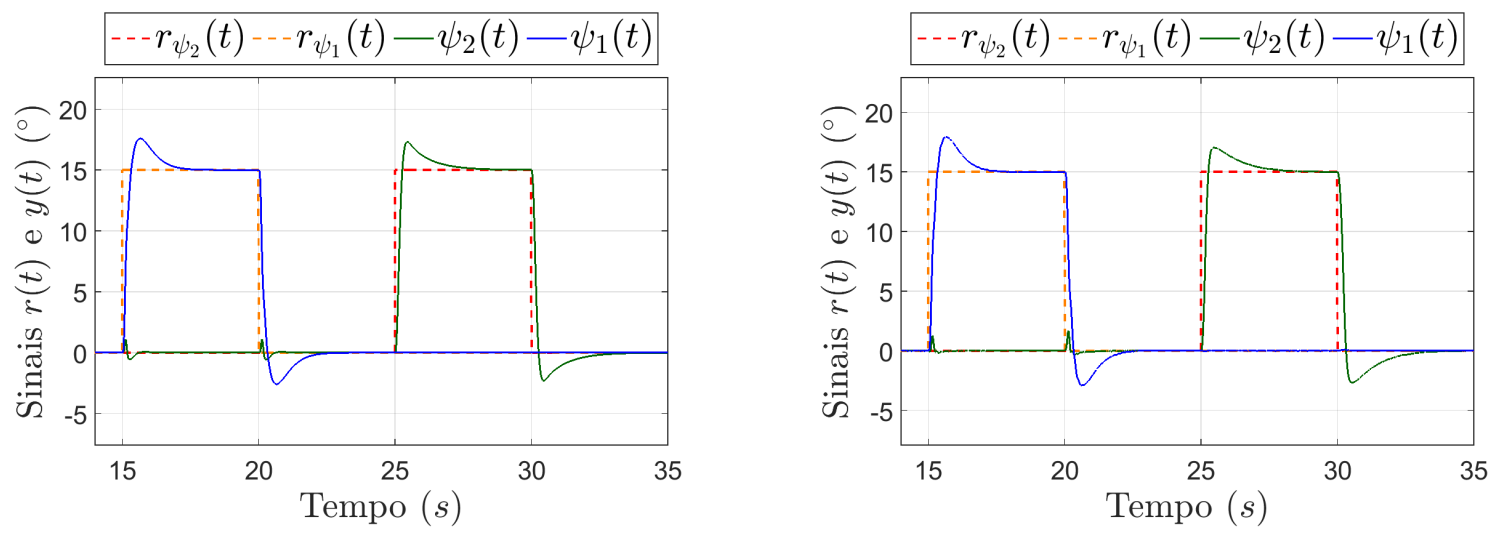

(c) Sinais de Controle $u(t)$ em N.m

(d) Sinais de Controle $u(t)$ em N.m
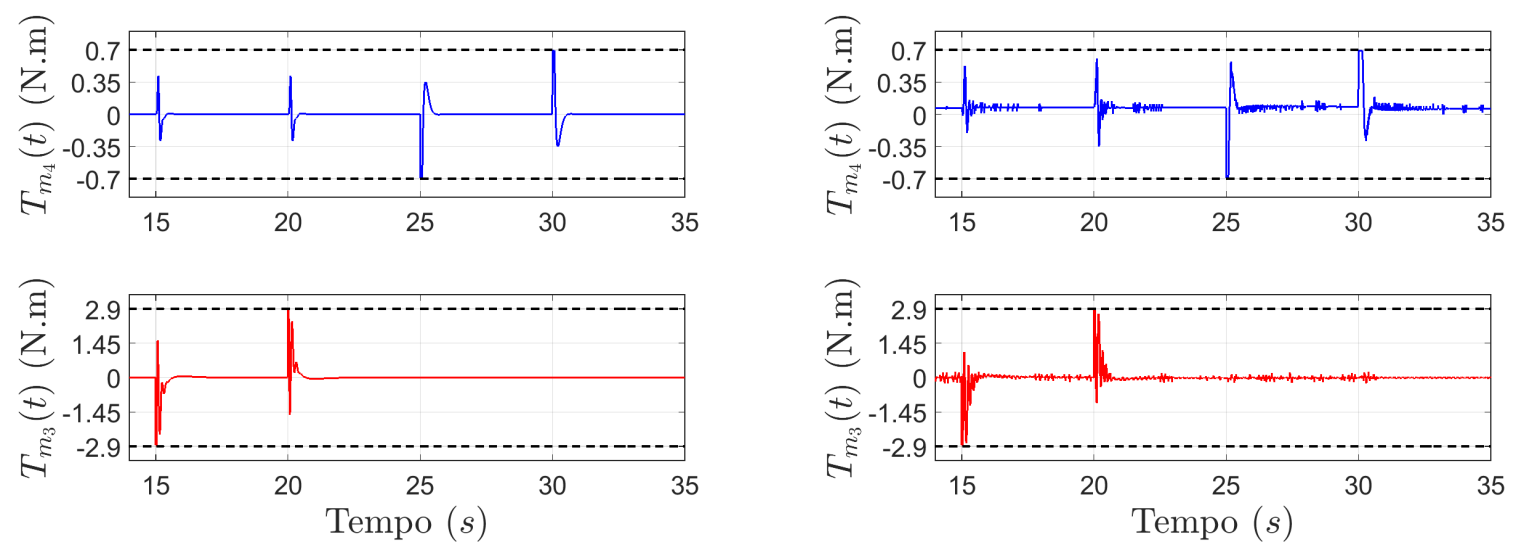

(e) Velocidades Angulares $\omega\left(k T_{s}\right)$ em $\% / \mathrm{s}$

(f) Velocidades Angulares $\omega\left(k T_{s}\right)$ em $\% / \mathrm{s}$
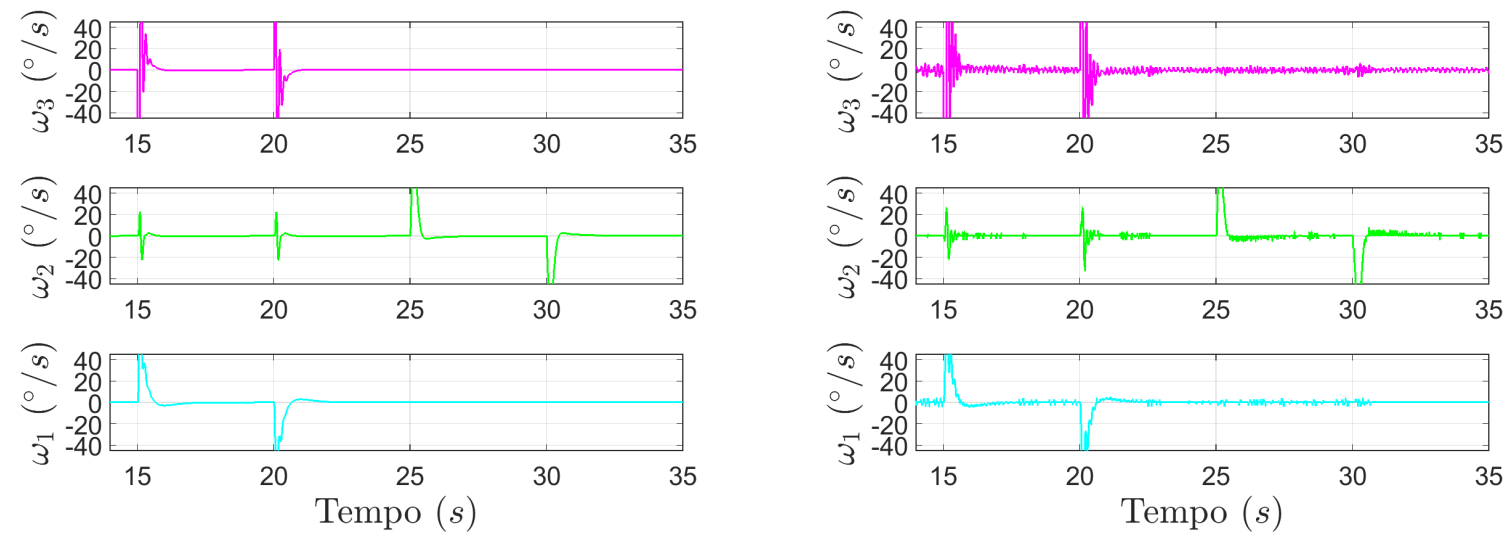

Fonte: Autor. 
Figura 29: Gráficos com os resultados do teste de acompanhamento de referência com sinais do ensaio 1 (negativo) - Sistema desacoplado com controle PID, com os sinais obtidos na simulação [(a), (c) e (e)] e no experimento [(b), (d) e (f)]

(a) Sinais $r(t)$ e $y(t)$ em $^{\circ}$

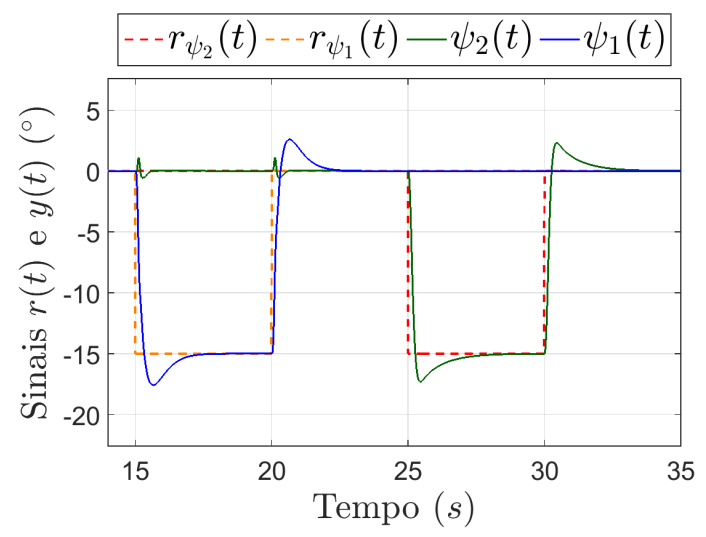

(c) Sinais de Controle $u(t)$ em N.m
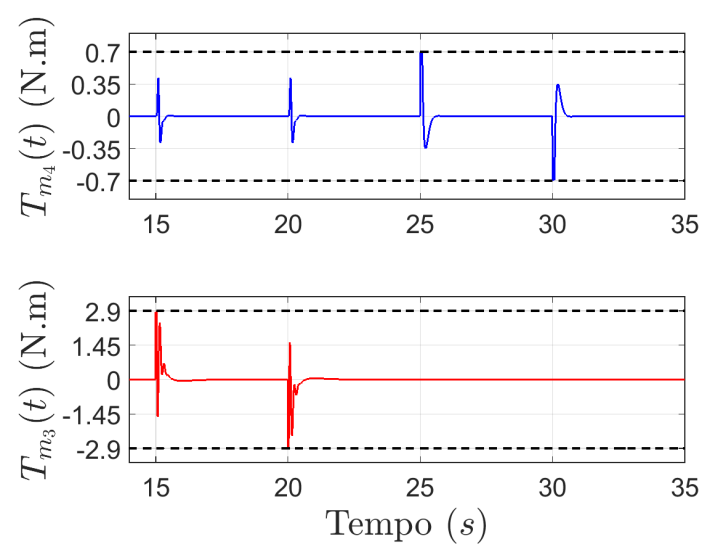

(e) Velocidades Angulares $\omega\left(k T_{s}\right)$ em $\% / \mathrm{s}$
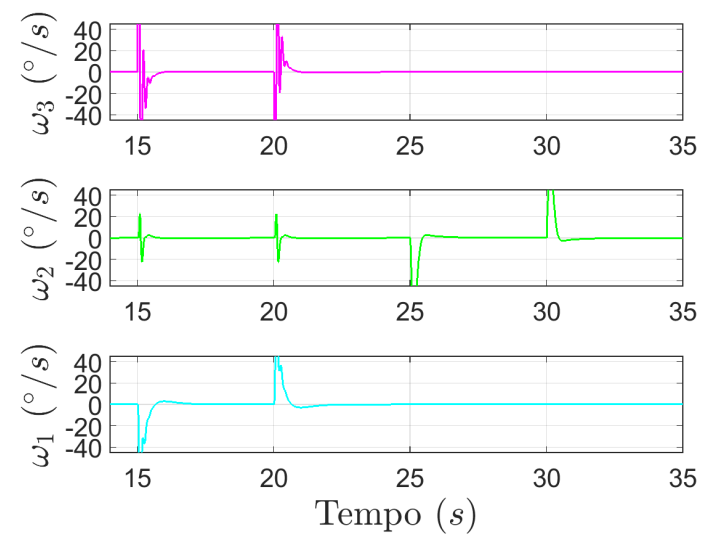

(b) Sinais $r(t)$ e $y(t) \mathrm{em}^{\circ}$

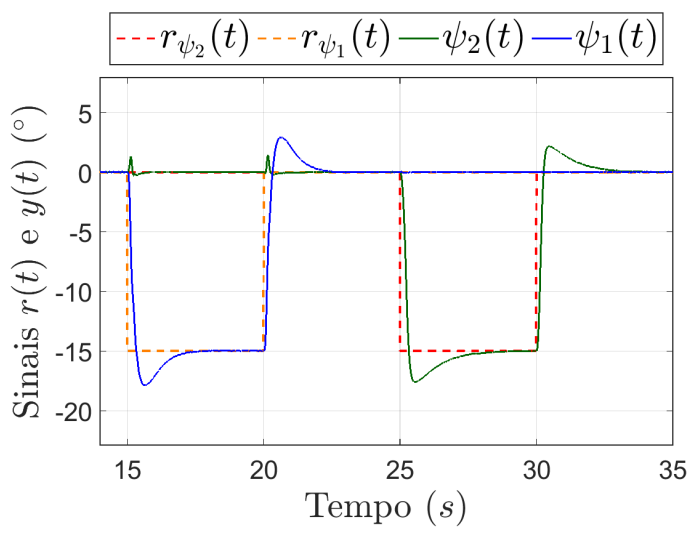

(d) Sinais de Controle $u(t)$ em N.m
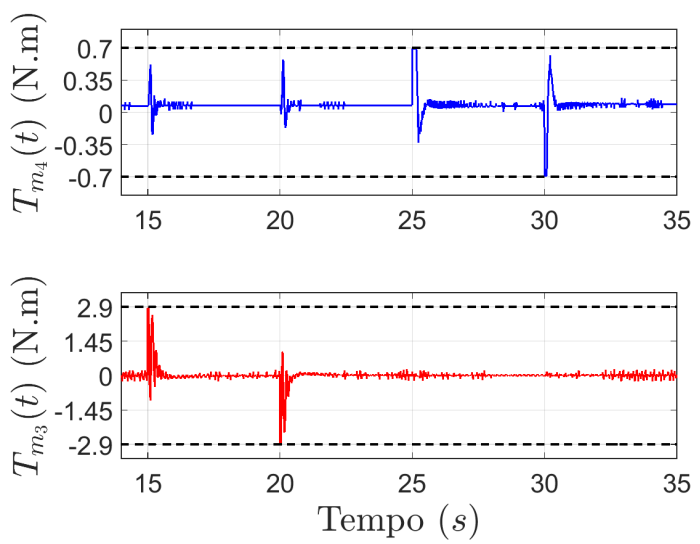

(f) Velocidades Angulares $\omega\left(k T_{s}\right)$ em $\% / \mathrm{s}$
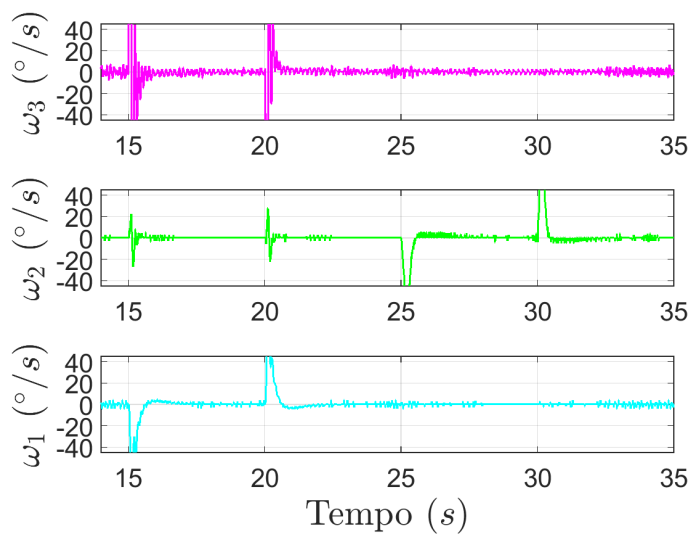

Fonte: Autor. 
Figura 30: Gráficos com os resultados do teste de rejeição à perturbação com sinais do ensaio 1 (positivo) - Sistema desacoplado com controle PID, com os sinais obtidos na simulação [(a), (c) e (e)] e no experimento [(b), (d) e (f)]

(a) Sinais $r(t)$ e $y(t) \mathrm{em}^{\circ}$

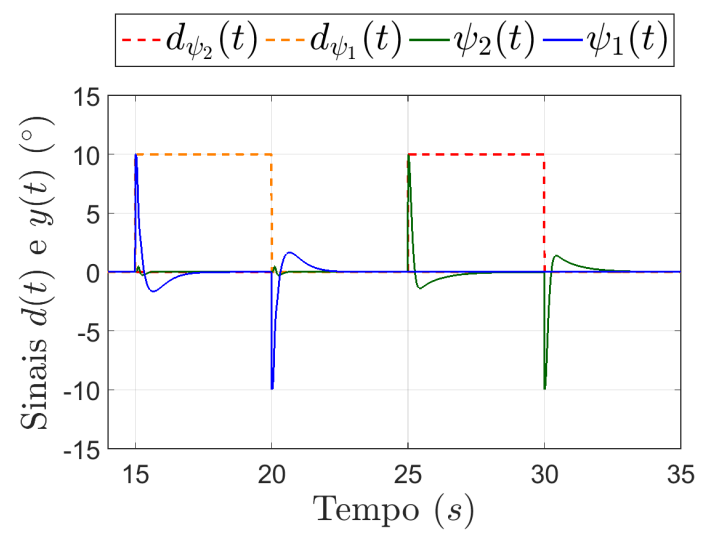

(c) Sinais de Controle $u(t)$ em N.m
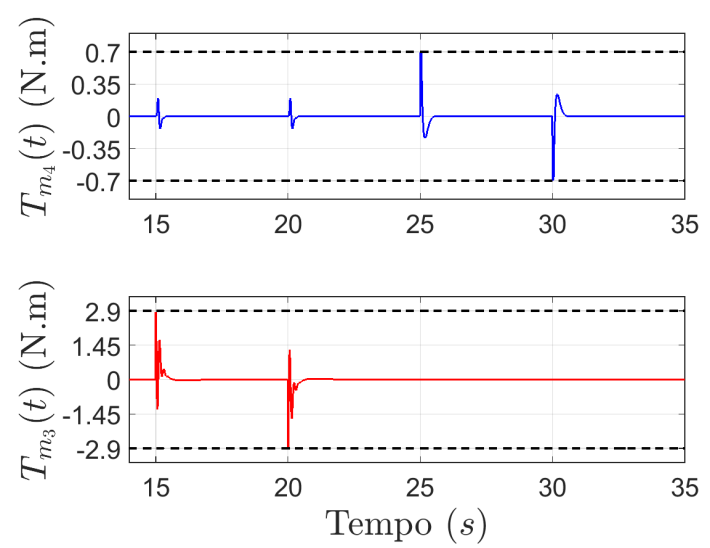

(e) Velocidades Angulares $\omega\left(k T_{s}\right)$ em $\% / \mathrm{s}$
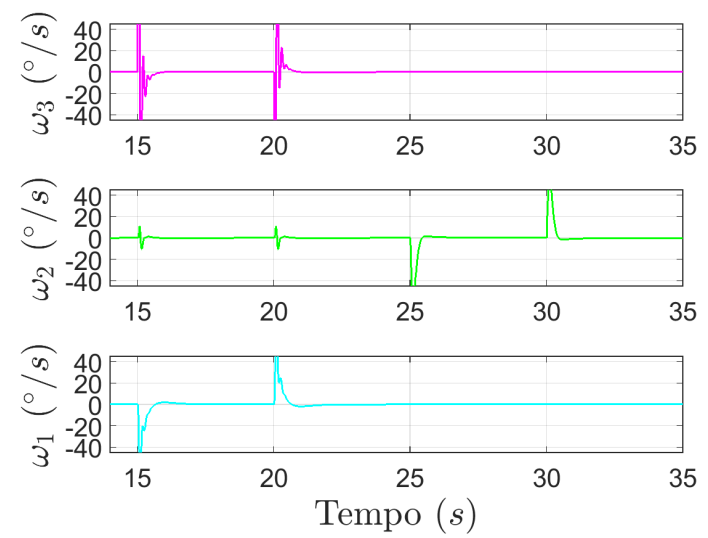

(b) Sinais $r(t)$ e $y(t)$ em $^{\circ}$

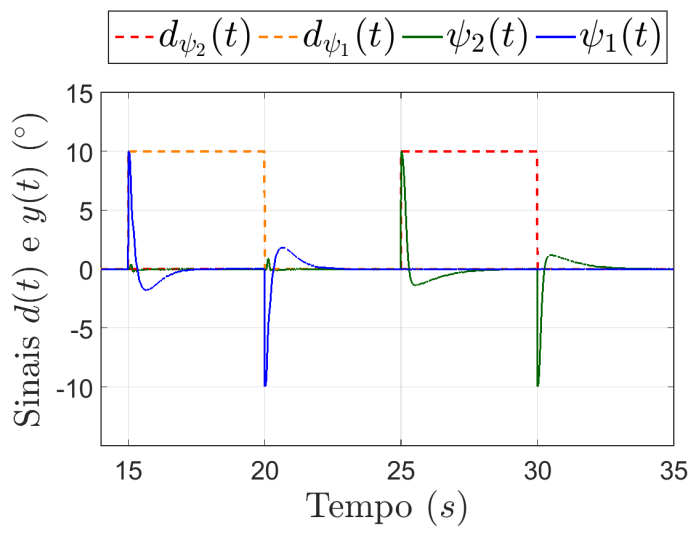

(d) Sinais de Controle $u(t)$ em N.m
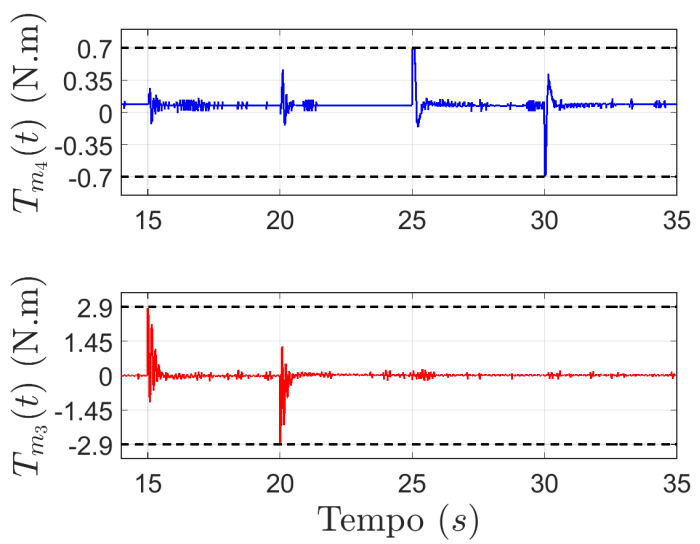

(f) Velocidades Angulares $\omega\left(k T_{s}\right)$ em $\% / \mathrm{s}$
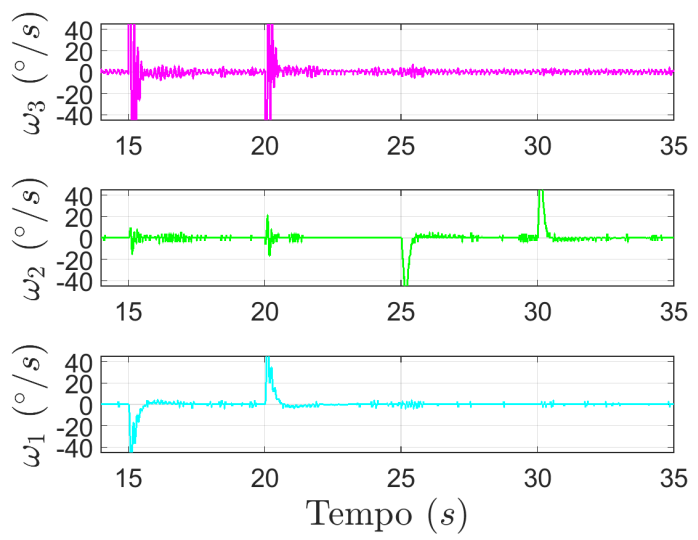

Fonte: Autor. 
Figura 31: Gráficos com os resultados do teste de rejeição à perturbação com sinais do ensaio 1 (negativo) - Sistema desacoplado com controle PID, com os sinais obtidos na simulação [(a), (c) e (e)] e no experimento [(b), (d) e (f)]
(a) Sinais $r(t)$ e $y(t)$ em $^{\circ}$
(b) Sinais $r(t)$ e $y(t) \mathrm{em}^{\circ}$
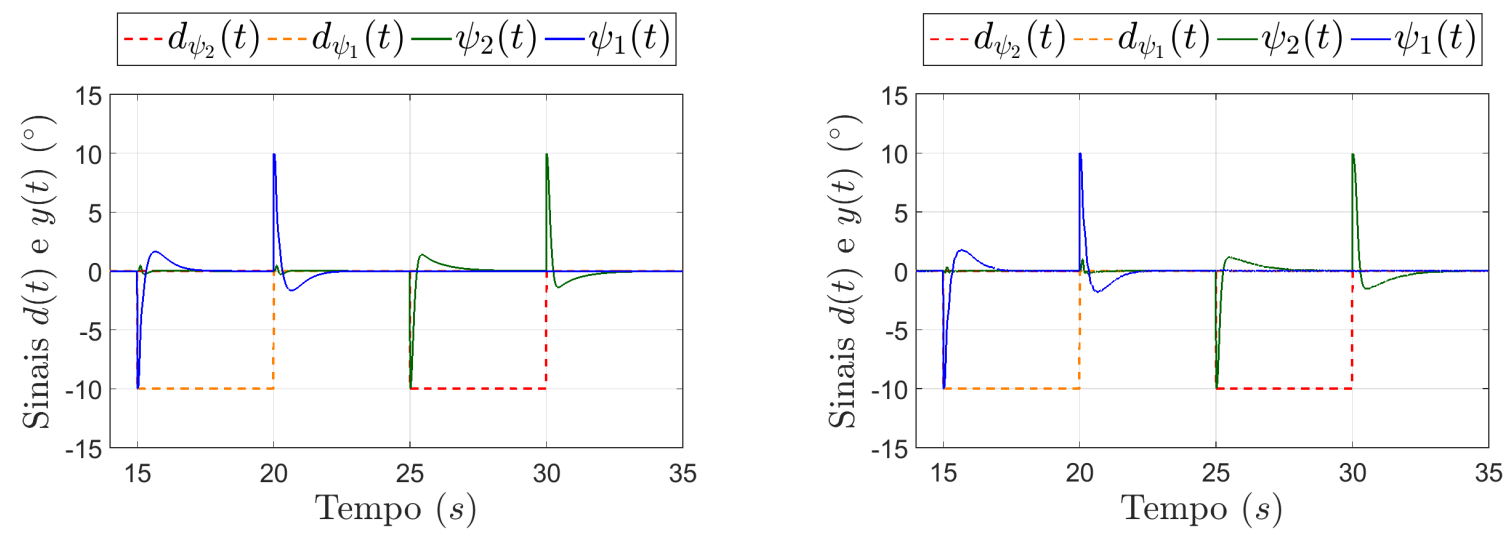

(c) Sinais de Controle $u(t)$ em N.m

(d) Sinais de Controle $u(t)$ em N.m
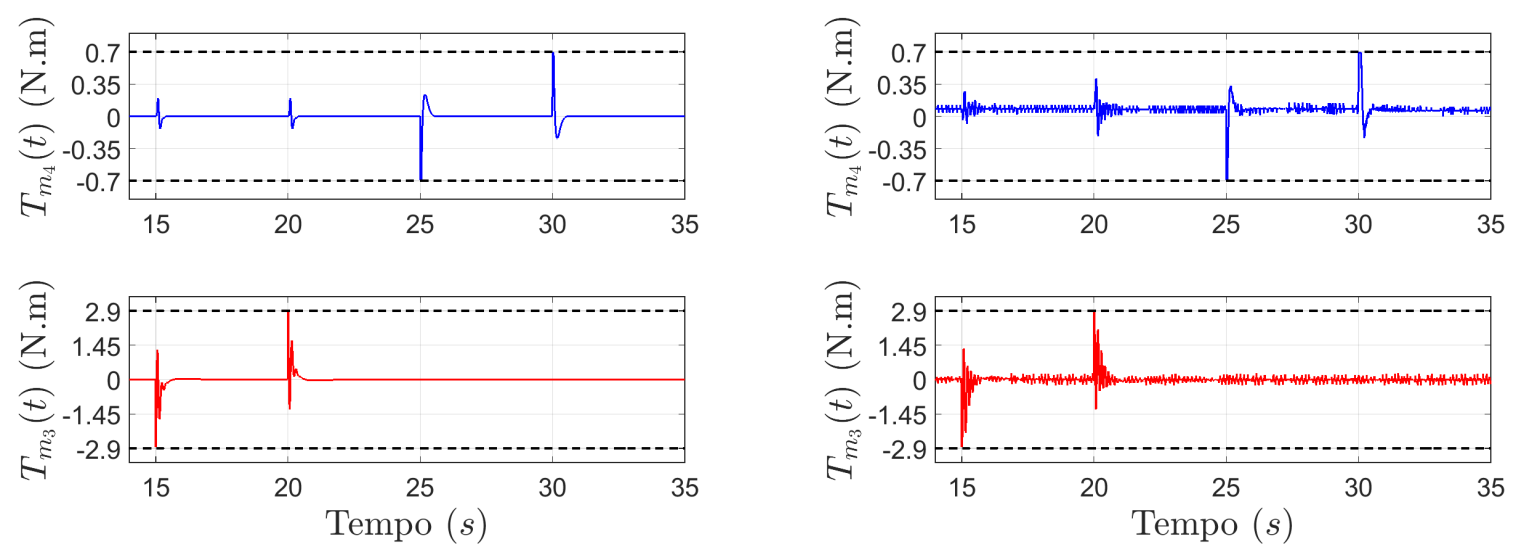

(e) Velocidades Angulares $\omega\left(k T_{s}\right)$ em $\% / \mathrm{s}$

(f) Velocidades Angulares $\omega\left(k T_{s}\right)$ em $\% / \mathrm{s}$
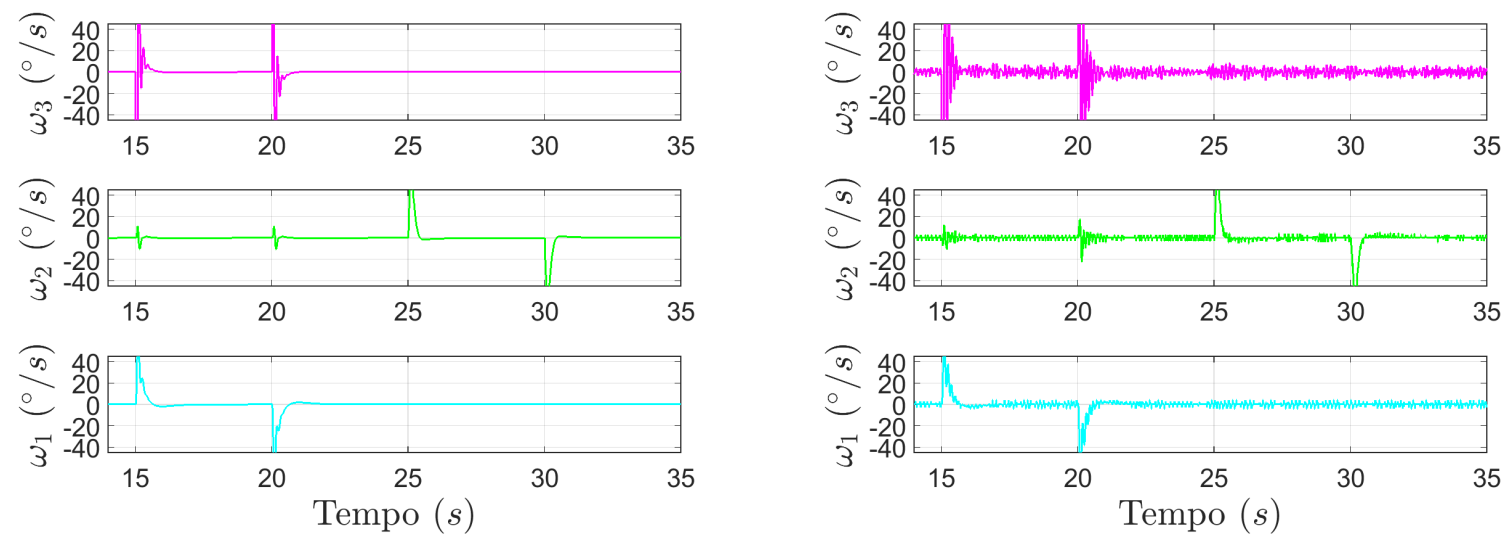

Fonte: Autor. 


\section{H.1.1.2 Ensaio 2 - Pulsos Retangulares Simultâneos}

As Figuras 32 e 33 apresentam os resultados obtidos nos testes de acompanhamento de referência e de rejeição à perturbação, respectivamente.

Figura 32: Gráficos com os resultados do teste de acompanhamento de referência com sinais do ensaio 2 - Sistema desacoplado com controle PID, com os sinais obtidos na simulação [(a), (c) e (e)] e no experimento [(b), (d) e (f)]

(a) Sinais $r(t)$ e $y(t) \mathrm{em}^{\circ}$

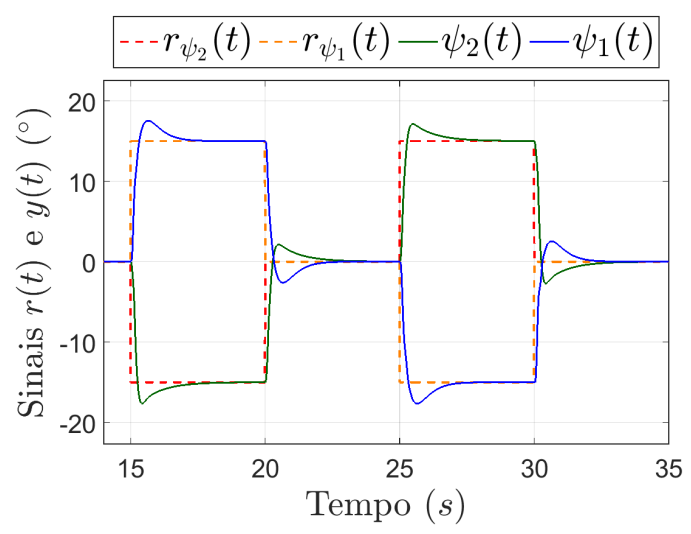

(c) Sinais de Controle $u(t)$ em N.m
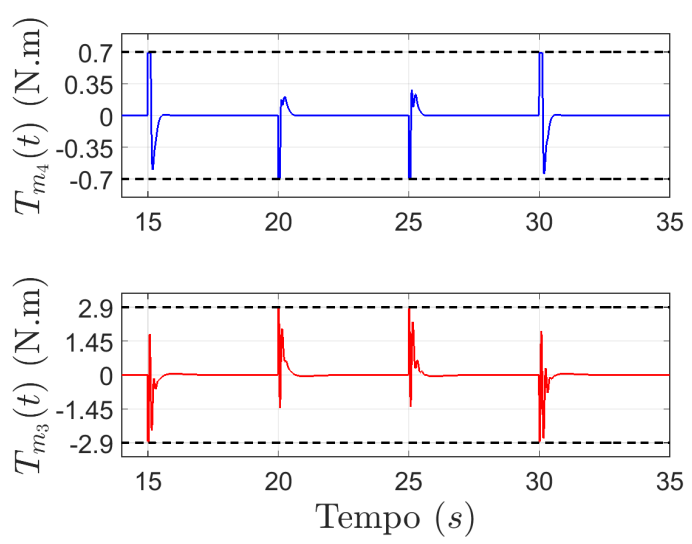

(e) Velocidades Angulares $\omega\left(k T_{s}\right) \mathrm{em}^{\circ} / \mathrm{s}$
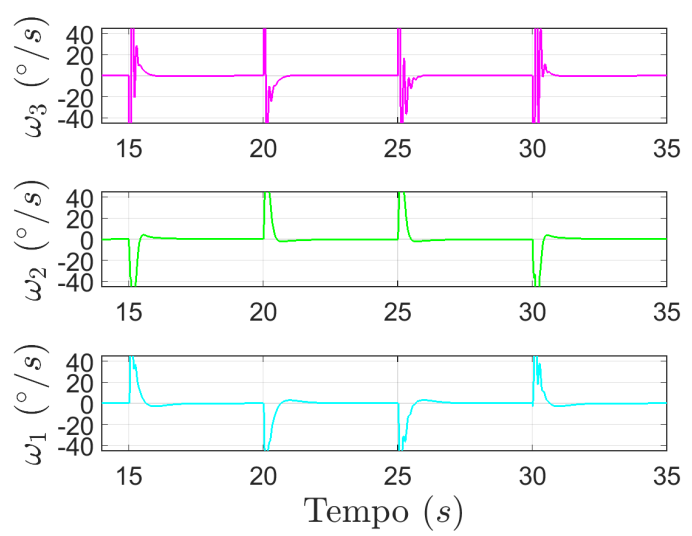

(b) Sinais $r(t)$ e $y(t) \mathrm{em}^{\circ}$

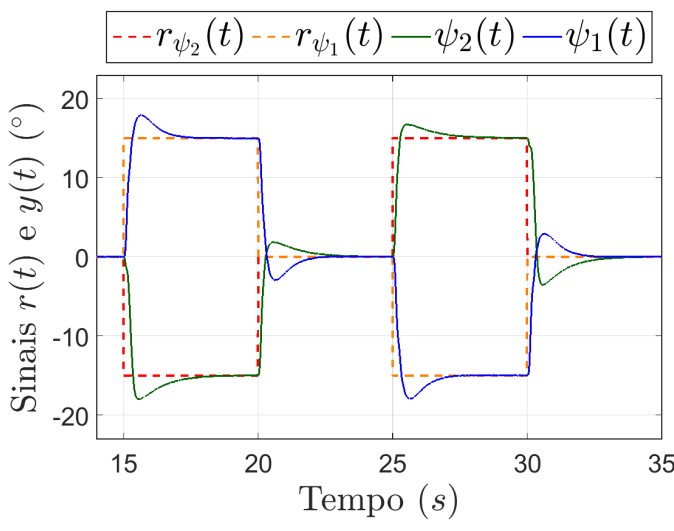

(d) Sinais de Controle $u(t)$ em N.m
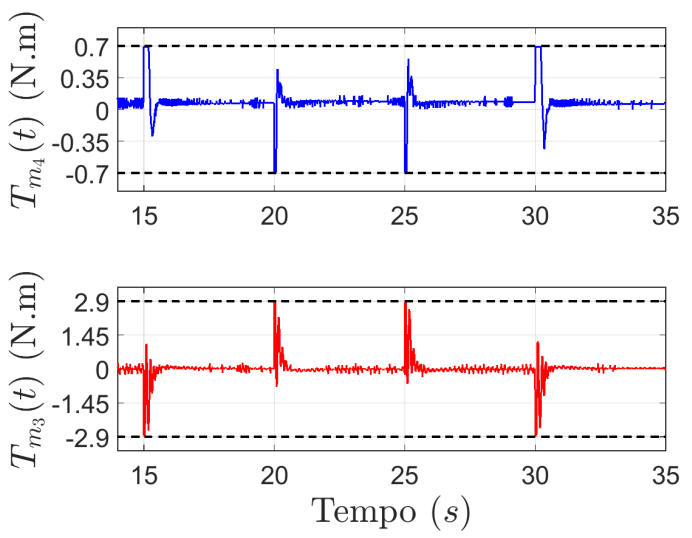

(f) Velocidades Angulares $\omega\left(k T_{s}\right)$ em $\% / \mathrm{s}$
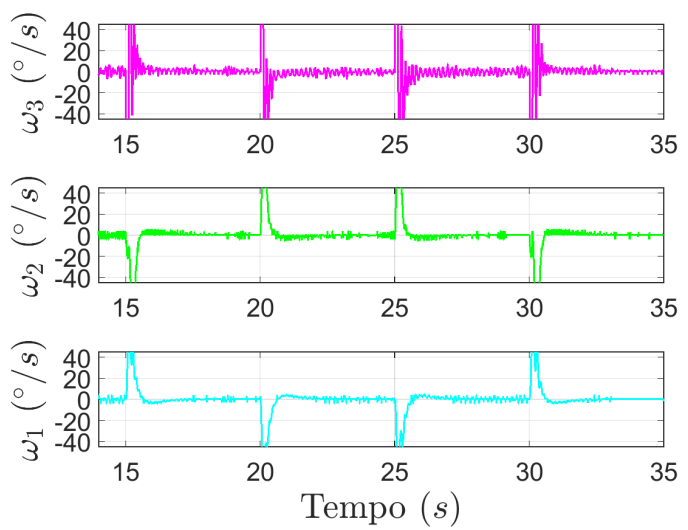

Fonte: Autor. 
Figura 33: Gráficos com os resultados do teste de rejeição à perturbação com sinais do ensaio 2 - Sistema desacoplado com controle PID, com os sinais obtidos na simulação [(a), (c) e (e)] e no experimento [(b), (d) e (f)]

(a) Sinais $r(t)$ e $y(t)$ em $^{\circ}$

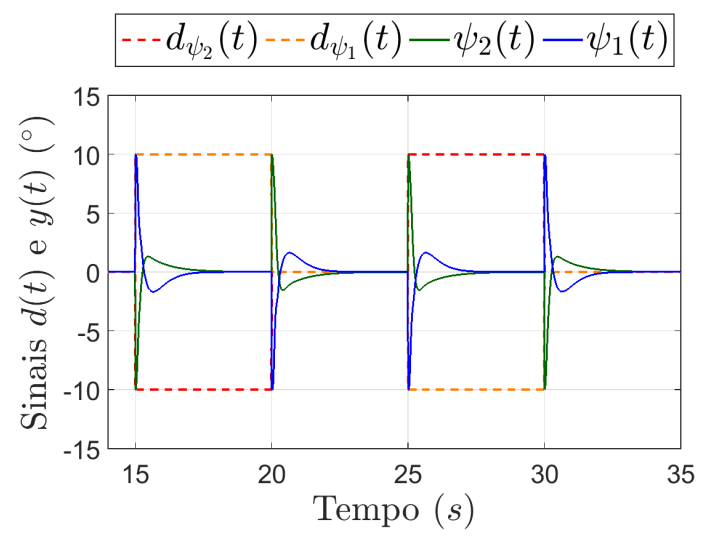

(c) Sinais de Controle $u(t)$ em N.m
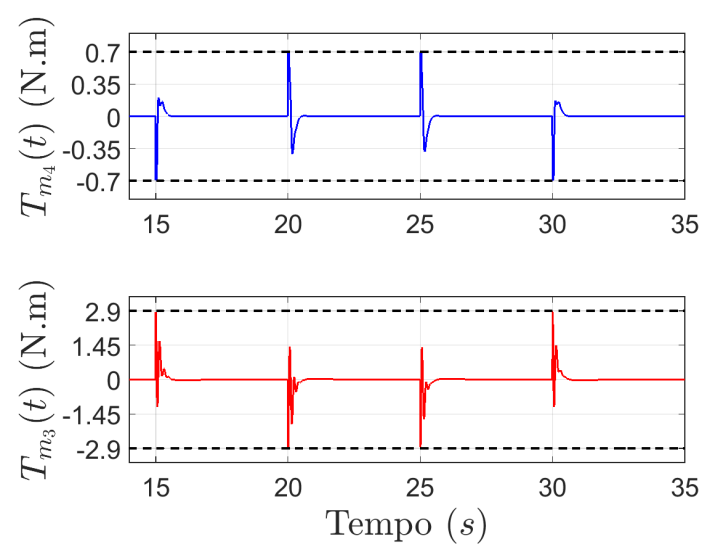

(e) Velocidades Angulares $\omega\left(k T_{s}\right) \mathrm{em} \% \mathrm{~s}$
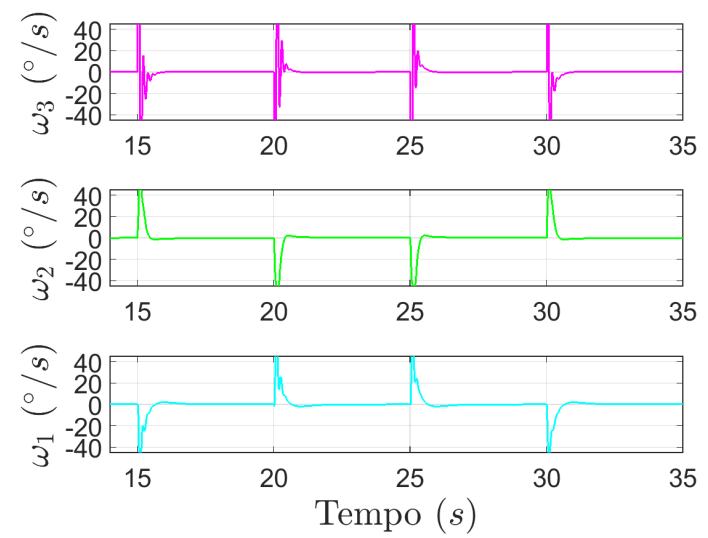

(b) Sinais $r(t)$ e $y(t)$ em $^{\circ}$

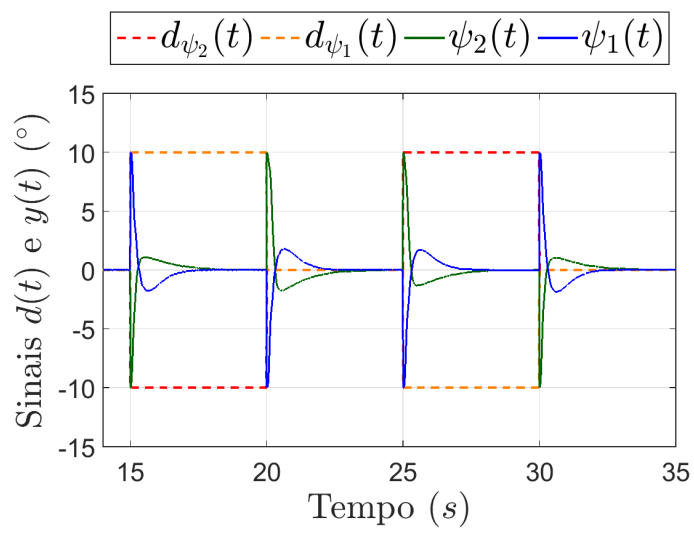

(d) Sinais de Controle $u(t)$ em N.m
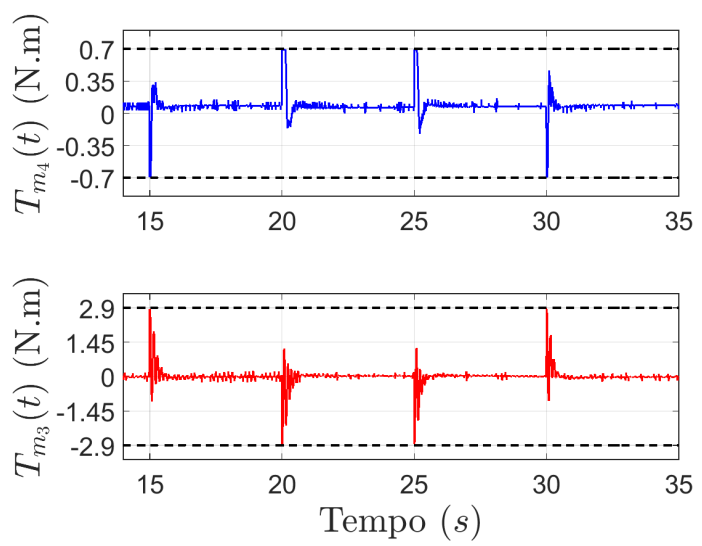

(f) Velocidades Angulares $\omega\left(k T_{s}\right)$ em $\% / \mathrm{s}$
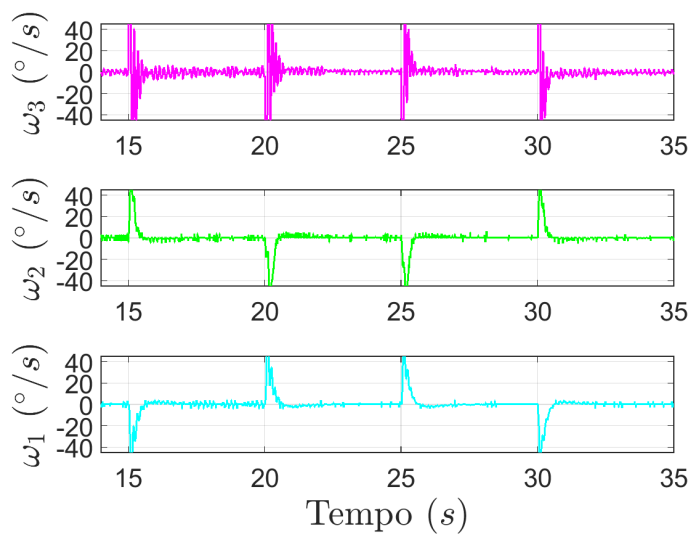

Fonte: Autor. 


\section{H.1.1.3 Ensaio 3 - Sinais Senoidais}

As Figuras 34 e 35 apresentam os resultados obtidos nos testes de acompanhamento de referência e de rejeição à perturbação, respectivamente.

Figura 34: Gráficos com os resultados do teste de acompanhamento de referência com sinais do ensaio 3 - Sistema desacoplado com controle PID, com os sinais obtidos na simulação [(a), (c) e (e)] e no experimento [(b), (d) e (f)]

(a) Sinais $r(t)$ e $y(t)$ em $^{\circ}$

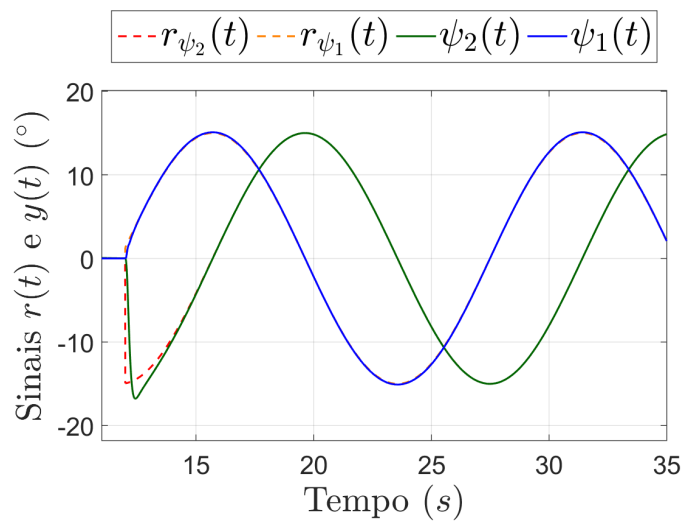

(c) Sinais de Controle $u(t)$ em N.m
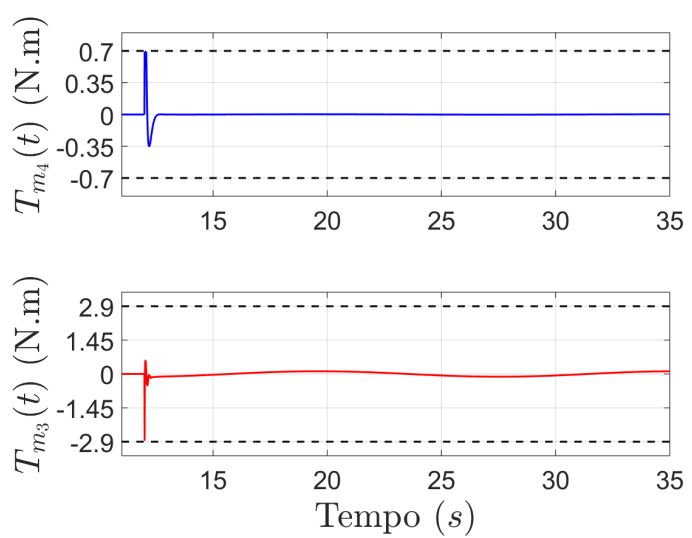

(e) Velocidades Angulares $\omega\left(k T_{s}\right) \mathrm{em} \% \mathrm{~s}$
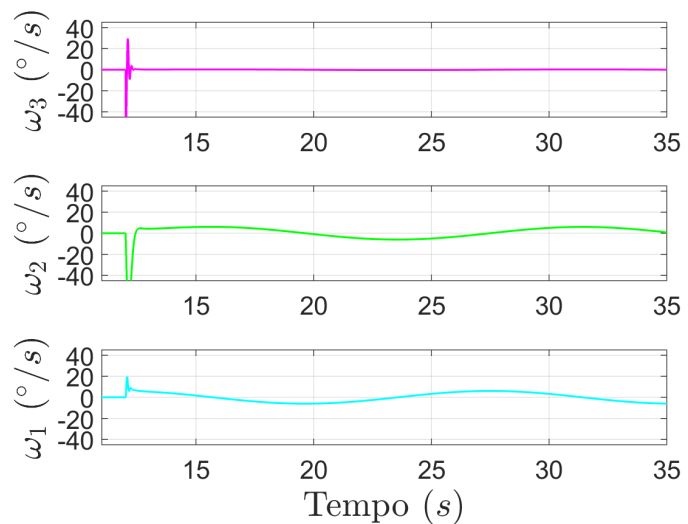

(b) Sinais $r(t)$ e $y(t) \mathrm{em}^{\circ}$

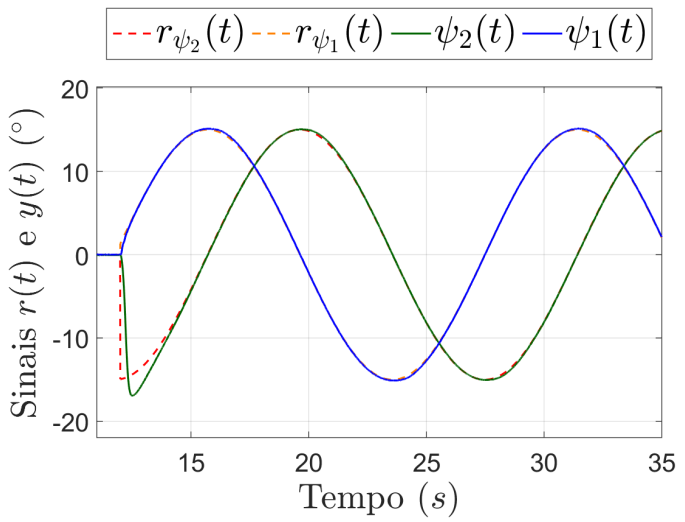

(d) Sinais de Controle $u(t)$ em N.m
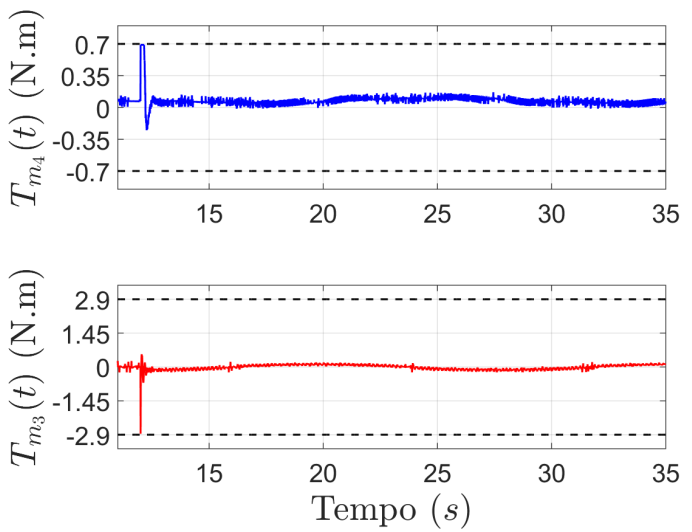

(f) Velocidades Angulares $\omega\left(k T_{s}\right)$ em \% $/ \mathrm{s}$
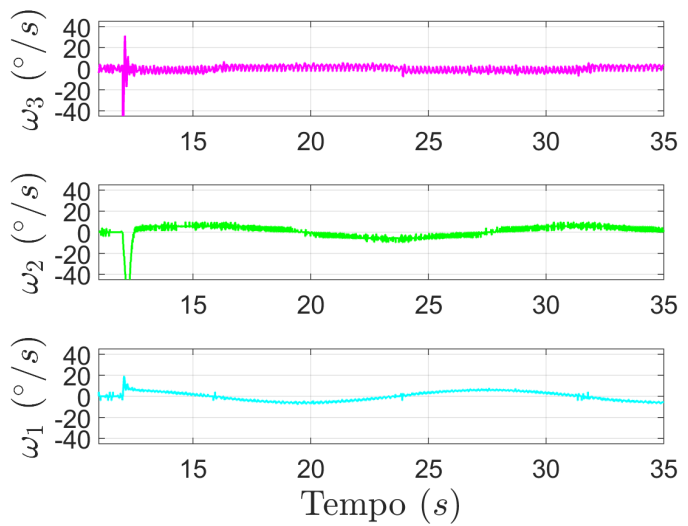

Fonte: Autor. 
Figura 35: Gráficos com os resultados do teste de rejeição à perturbação com sinais do ensaio 3 - Sistema desacoplado com controle PID, com os sinais obtidos na simulação [(a), (c) e (e)] e no experimento [(b), (d) e (f)]
(a) Sinais $r(t)$ e $y(t) \mathrm{em}^{\circ}$
(b) Sinais $r(t)$ e $y(t) \mathrm{em}^{\circ}$

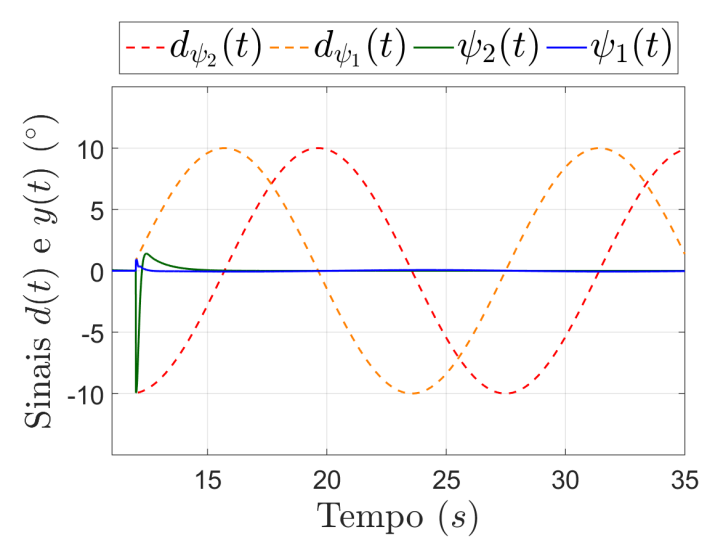

(c) Sinais de Controle $u(t)$ em N.m
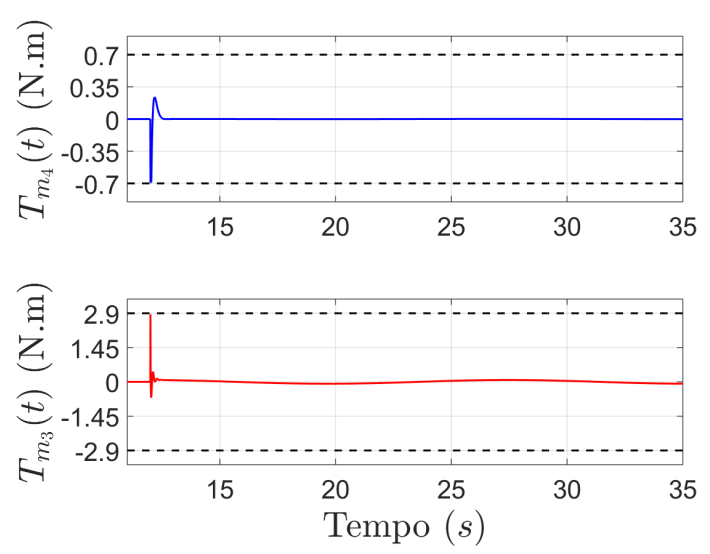

(e) Velocidades Angulares $\omega\left(k T_{s}\right) \mathrm{em} \% \mathrm{~s}$
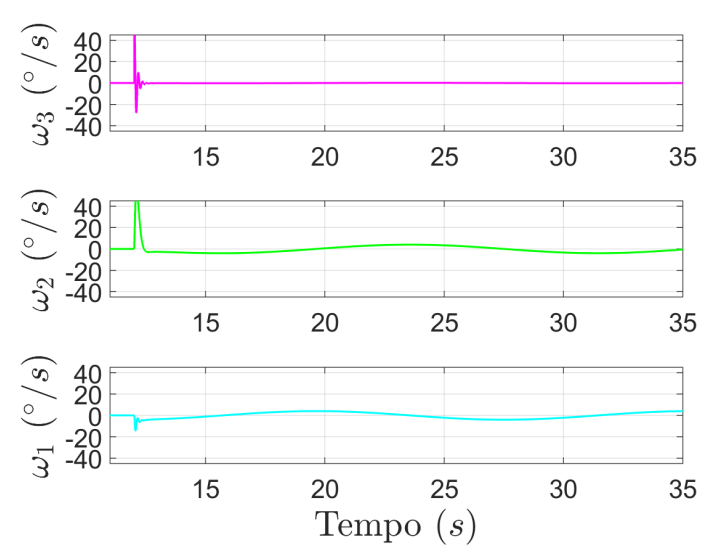

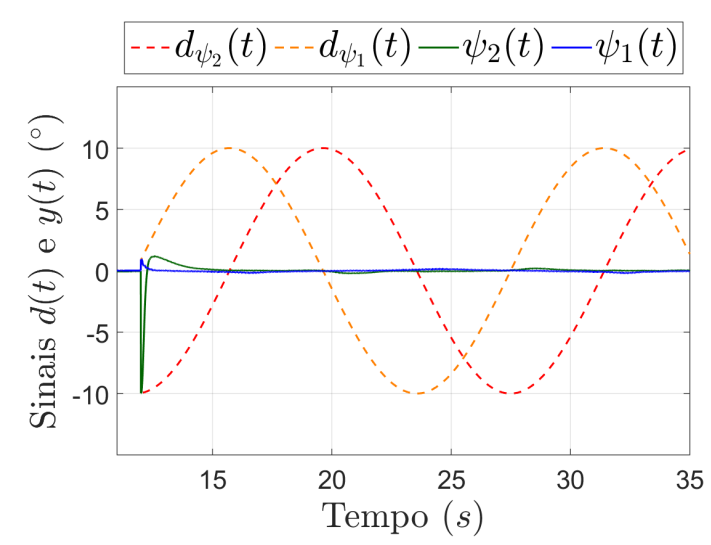

(d) Sinais de Controle $u(t)$ em N.m
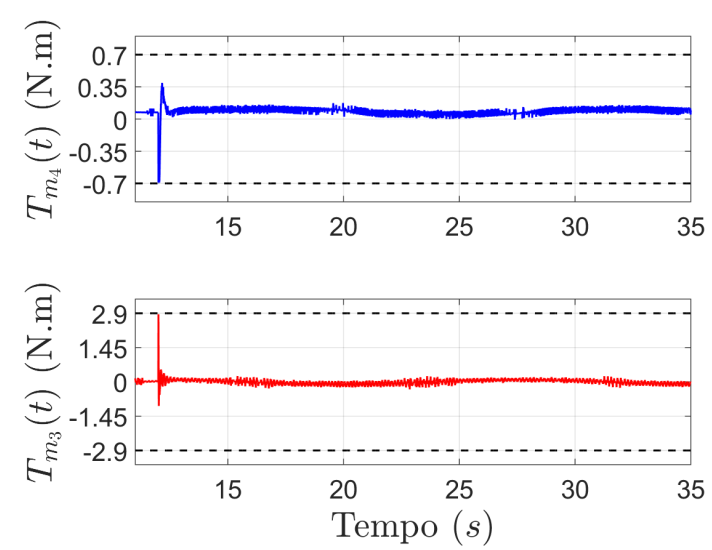

(f) Velocidades Angulares $\omega\left(k T_{s}\right) \mathrm{em} \% \mathrm{~s}$
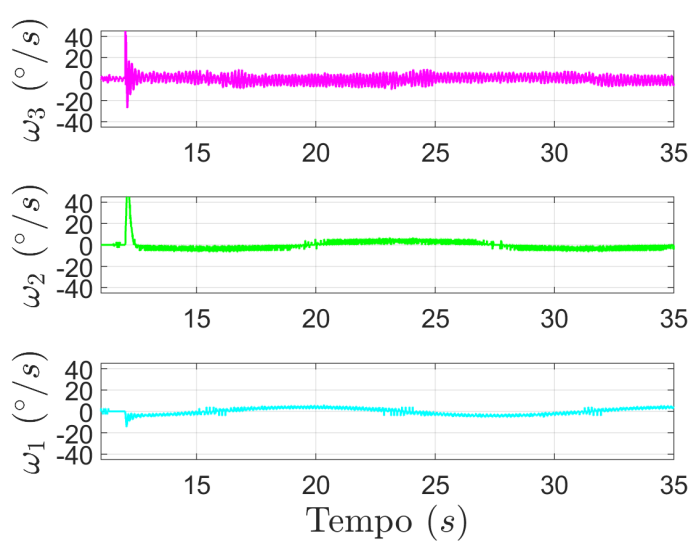

Fonte: Autor. 


\section{H.1.2 Sistema com o Controlador LQR}

Para o sistema desacoplado, o controlador digital LQR utilizado é projetado em relação ao modelo desacoplado da planta. Os resultados obtidos para os 3 ensaios de sinais considerados são apresentados a seguir.

\section{H.1.2.1 Ensaio 1 - Pulsos Retangulares Separados}

As Figuras 36 e 37 apresentam os resultados obtidos no teste de acompanhamento de referência com amplitudes positivas e negativas, respectivamente. e as Figuras 38 e 39 os resultados obtidos no teste de rejeição à perturbação com amplitudes positivas e negativas, respectivamente. 
Figura 36: Gráficos com os resultados do teste de acompanhamento de referência com sinais do ensaio 1 (positivo) - Sistema desacoplado com controle LQR, com os sinais obtidos na simulação [(a), (c) e (e)] e no experimento [(b), (d) e (f)]

(a) Sinais $r(t)$ e $y(t)$ em $^{\circ}$

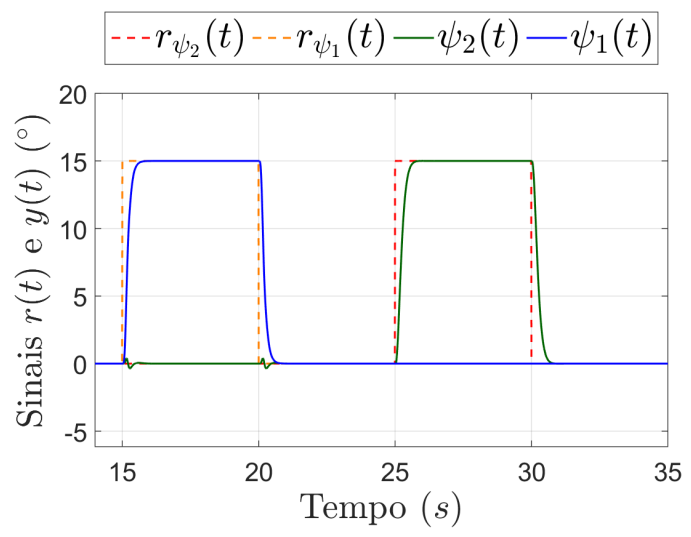

(c) Sinais de Controle $u(t)$ em N.m
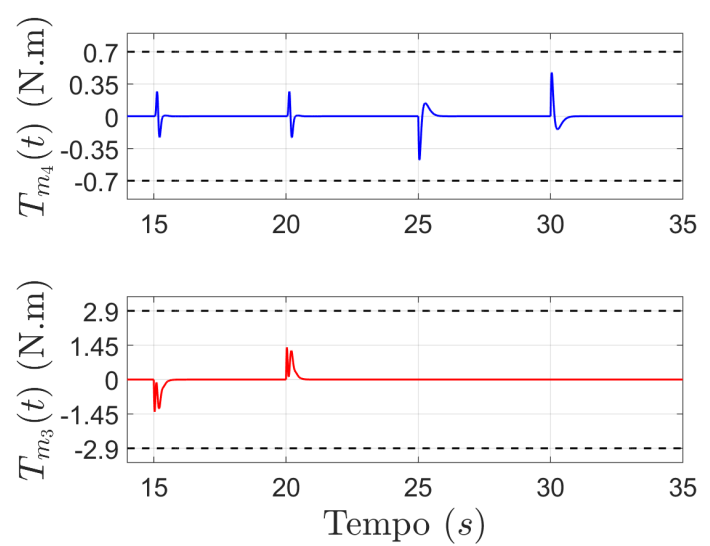

(e) Velocidades Angulares $\omega\left(k T_{s}\right)$ em $\% / \mathrm{s}$
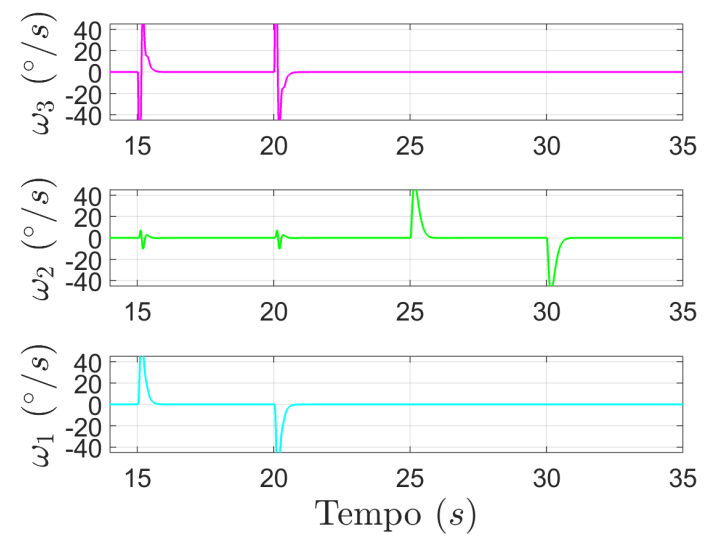

(b) Sinais $r(t)$ e $y(t)$ em $^{\circ}$

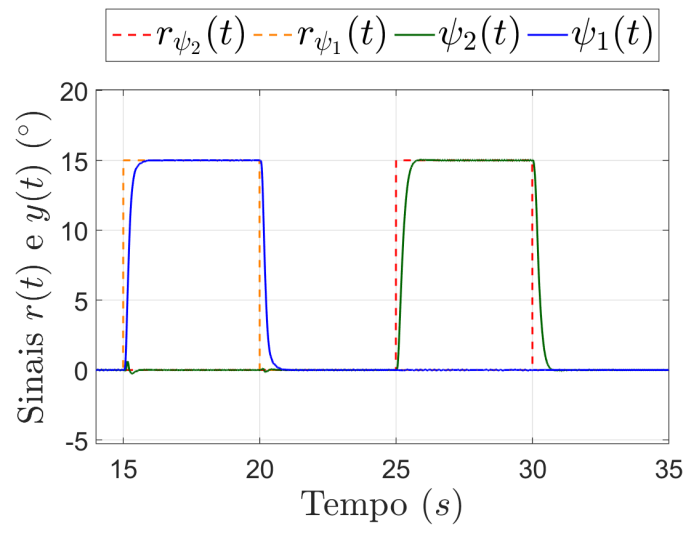

(d) Sinais de Controle $u(t)$ em N.m
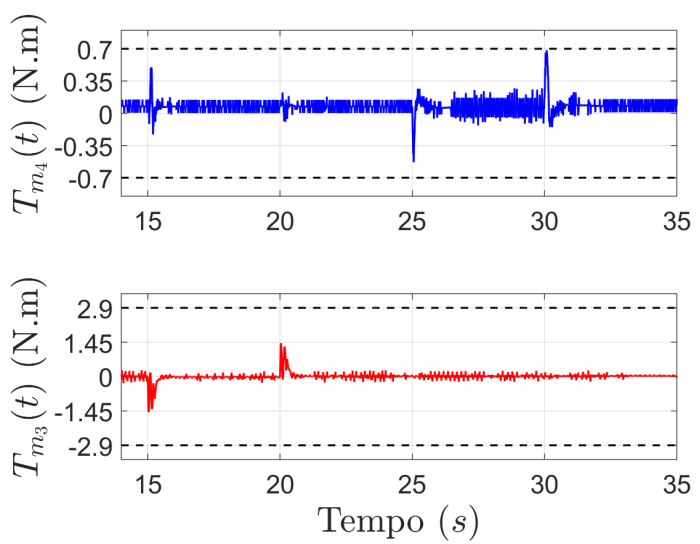

(f) Velocidades Angulares $\omega\left(k T_{s}\right)$ em $\% / \mathrm{s}$
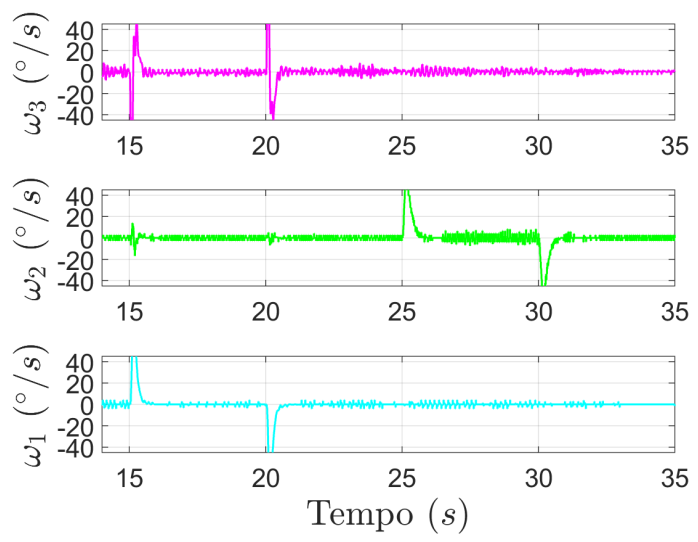

Fonte: Autor. 
Figura 37: Gráficos com os resultados do teste de acompanhamento de referência com sinais do ensaio 1 (negativo) - Sistema desacoplado com controle LQR, com os sinais obtidos na simulação [(a), (c) e (e)] e no experimento [(b), (d) e (f)]

(a) Sinais $r(t)$ e $y(t)$ em $^{\circ}$

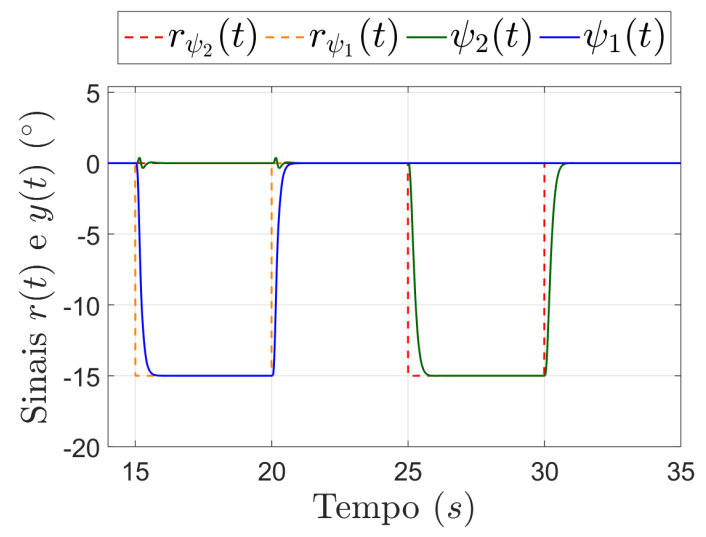

(c) Sinais de Controle $u(t)$ em N.m
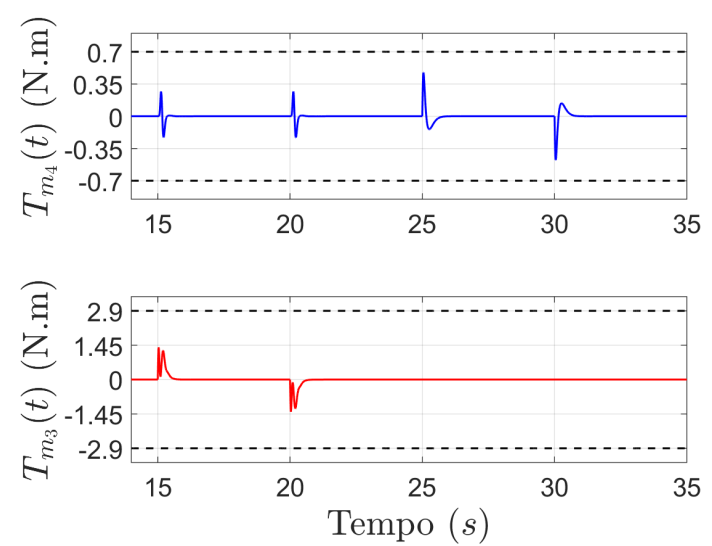

(e) Velocidades Angulares $\omega\left(k T_{s}\right)$ em $\% / \mathrm{s}$
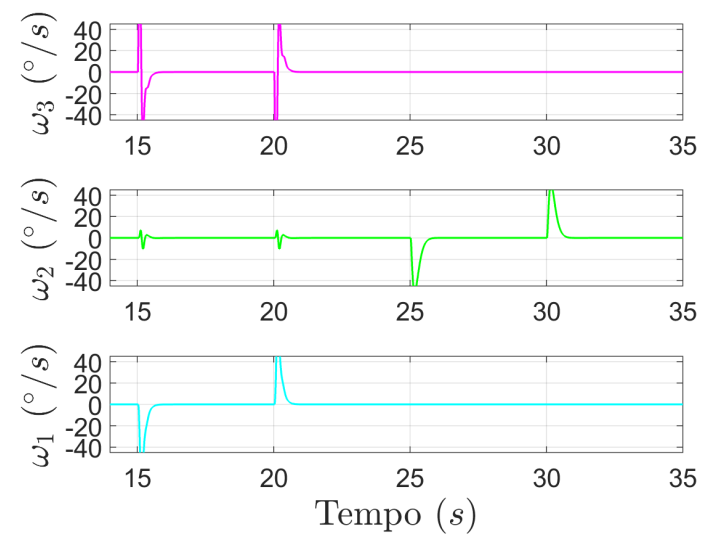

(b) Sinais $r(t)$ e $y(t) \mathrm{em}^{\circ}$

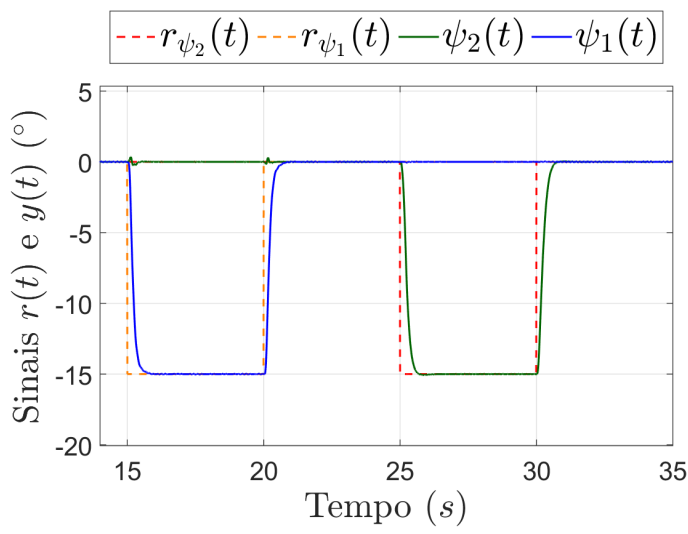

(d) Sinais de Controle $u(t)$ em N.m
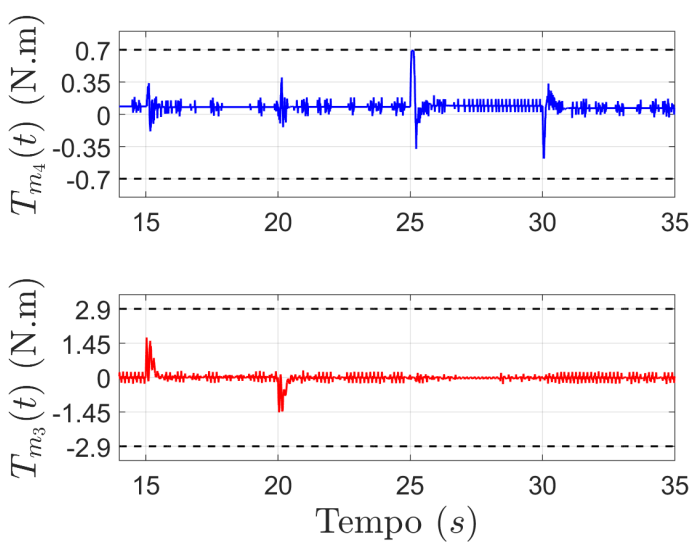

(f) Velocidades Angulares $\omega\left(k T_{s}\right)$ em $\% / \mathrm{s}$
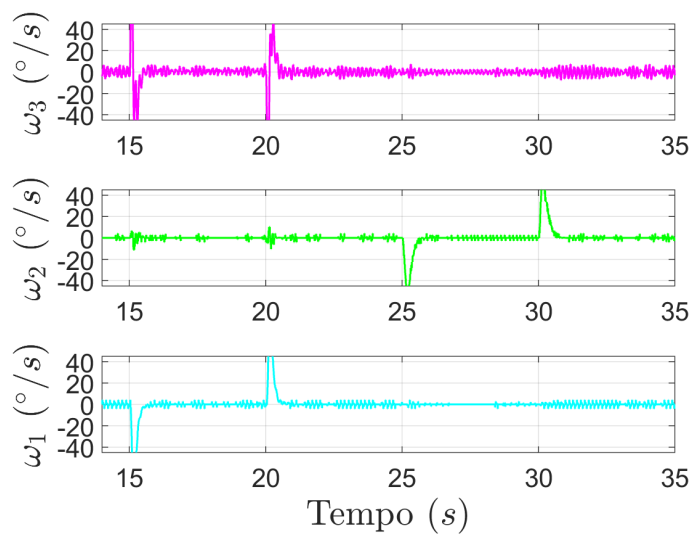

Fonte: Autor. 
Figura 38: Gráficos com os resultados do teste de rejeição à perturbação com sinais do ensaio 1 (positivo) - Sistema desacoplado com controle LQR, com os sinais obtidos na simulação [(a), (c) e (e)] e no experimento [(b), (d) e (f)]

(a) Sinais $r(t)$ e $y(t) \mathrm{em}^{\circ}$

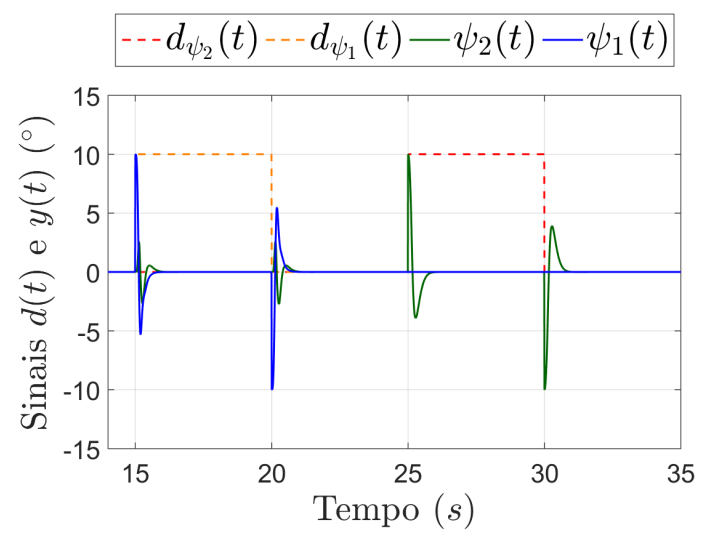

(c) Sinais de Controle $u(t)$ em N.m
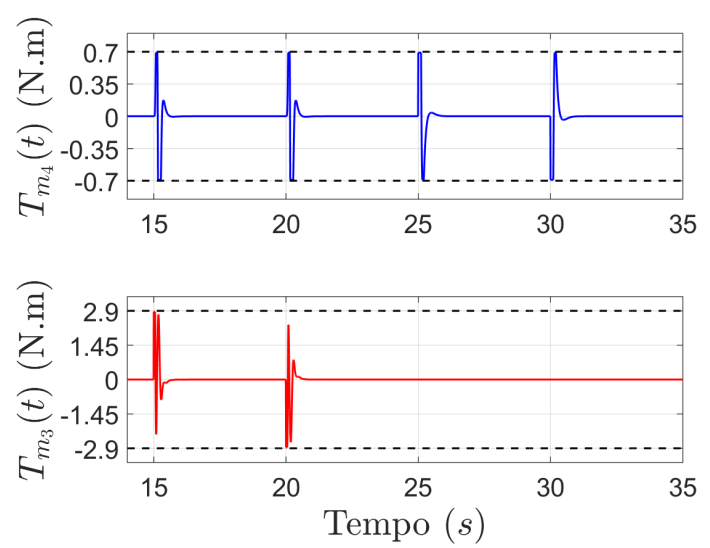

(e) Velocidades Angulares $\omega\left(k T_{s}\right) \mathrm{em} \% \mathrm{~s}$
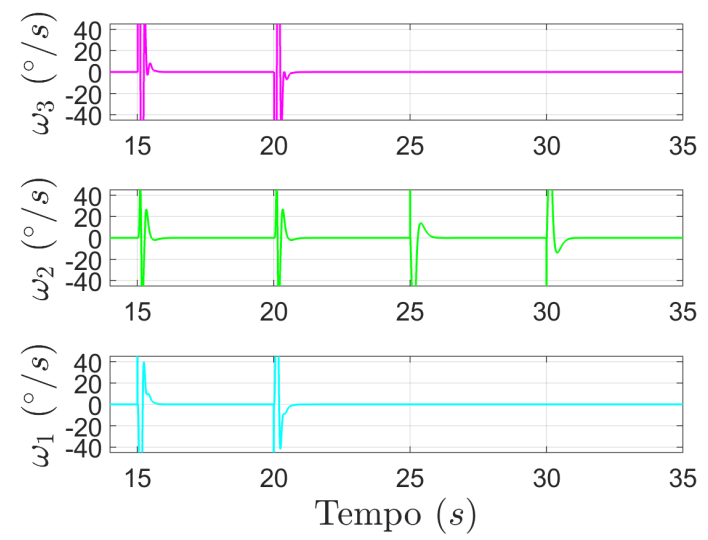

(b) Sinais $r(t)$ e $y(t) \mathrm{em}^{\circ}$

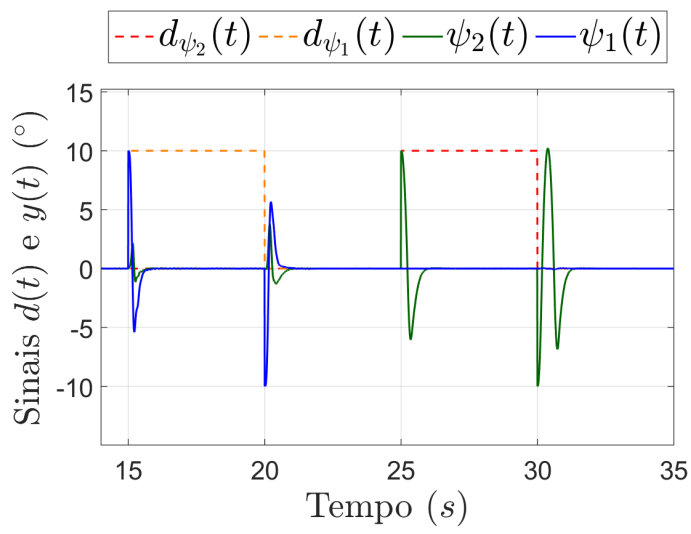

(d) Sinais de Controle $u(t)$ em N.m
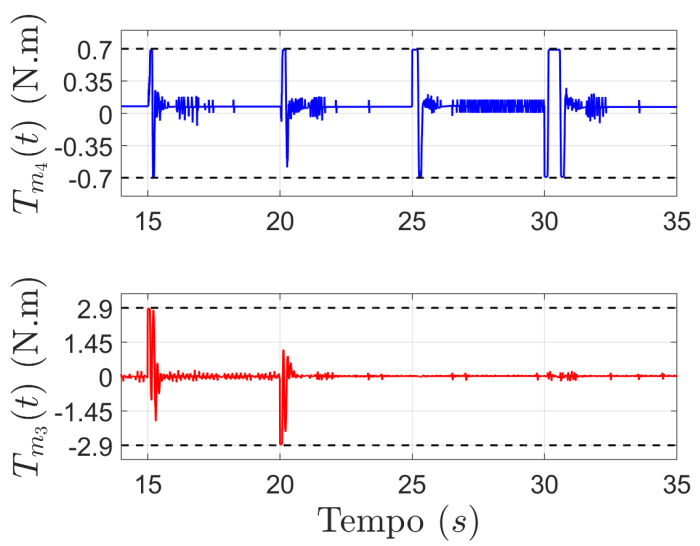

(f) Velocidades Angulares $\omega\left(k T_{s}\right)$ em $\% / \mathrm{s}$
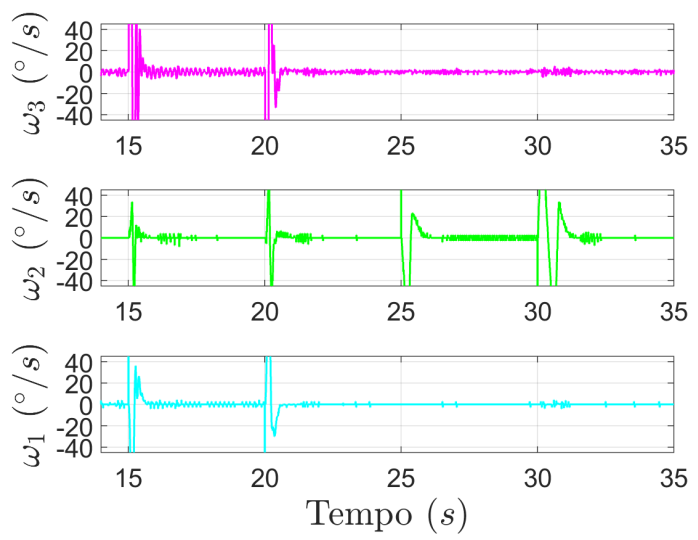

Fonte: Autor. 
Figura 39: Gráficos com os resultados do teste de rejeição à perturbação com sinais do ensaio 1 (negativo) - Sistema desacoplado com controle LQR, com os sinais obtidos na simulação [(a), (c) e (e)] e no experimento [(b), (d) e (f)]
(a) Sinais $r(t)$ e $y(t) \mathrm{em}^{\circ}$
(b) Sinais $r(t)$ e $y(t) \mathrm{em}^{\circ}$
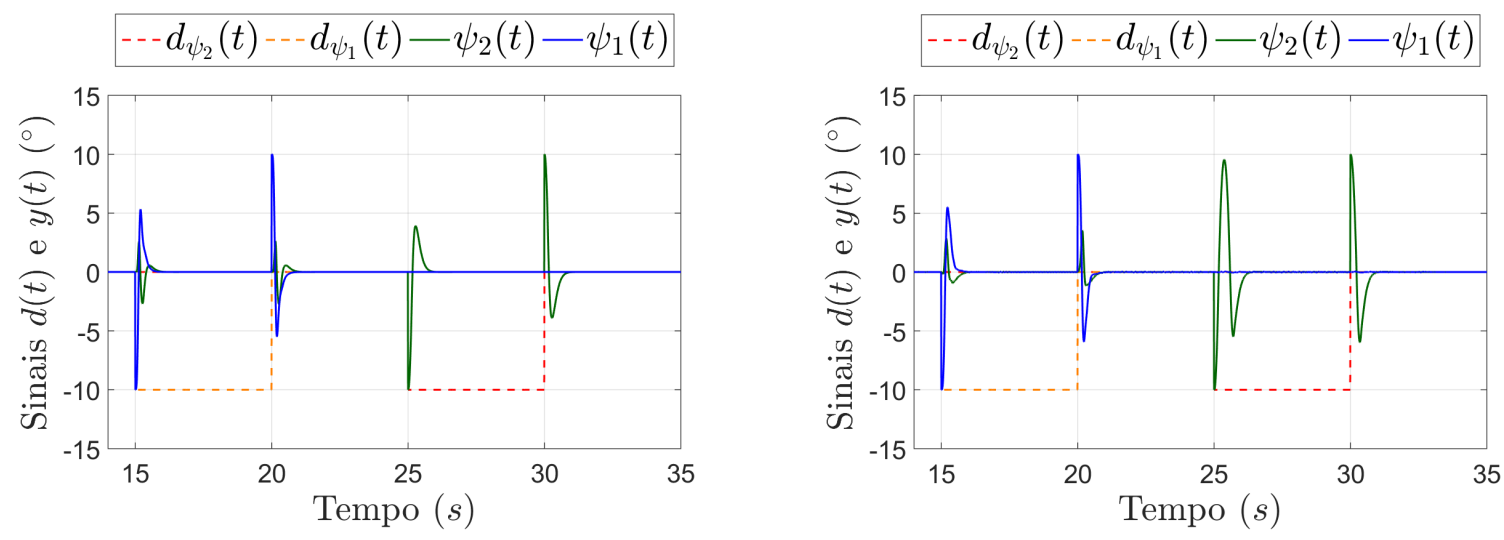

(c) Sinais de Controle $u(t)$ em N.m

(d) Sinais de Controle $u(t)$ em N.m
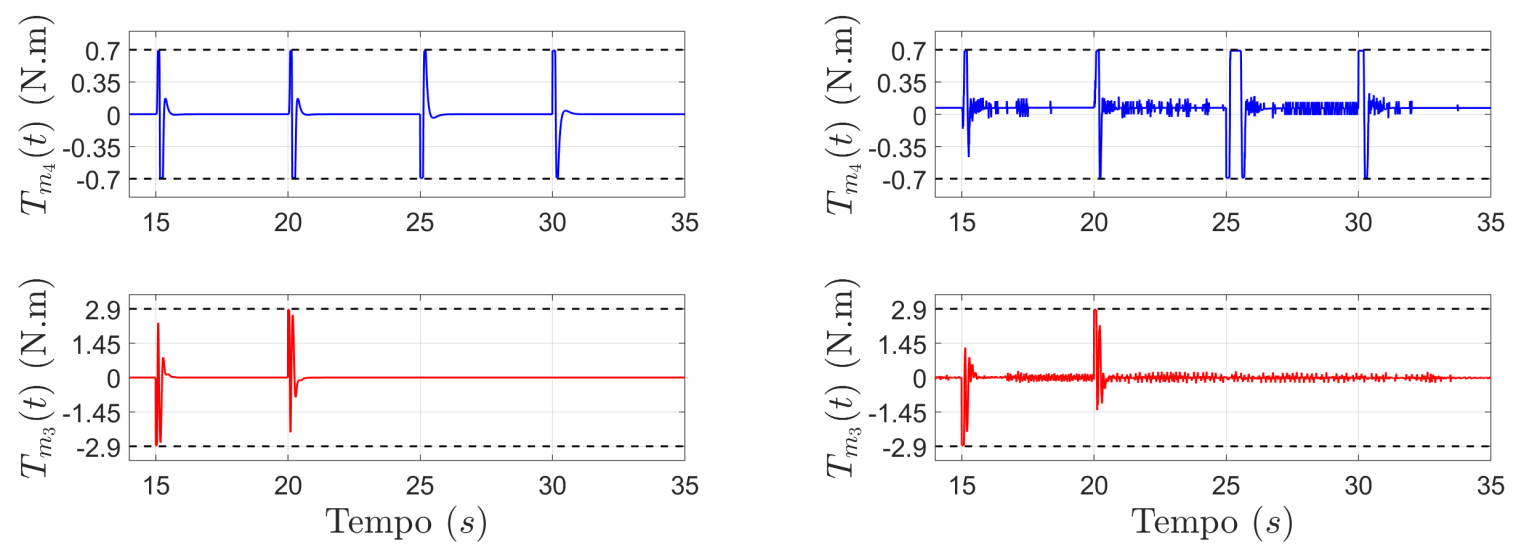

(e) Velocidades Angulares $\omega\left(k T_{s}\right)$ em $\% / \mathrm{s}$

(f) Velocidades Angulares $\omega\left(k T_{s}\right)$ em $\% / \mathrm{s}$
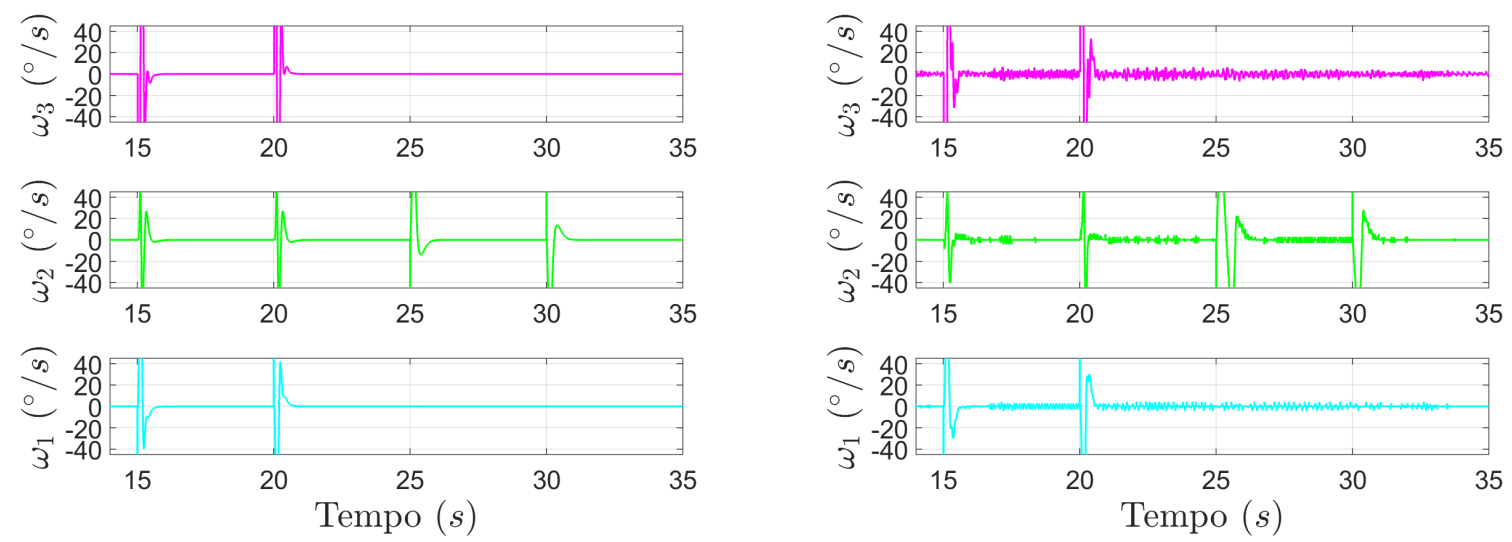

Fonte: Autor. 


\section{H.1.2.2 Ensaio 2 - Pulsos Retangulares Simultâneos}

As Figuras 40 e 41 apresentam os resultados obtidos nos testes de acompanhamento de referência e de rejeição à perturbação, respectivamente.

Figura 40: Gráficos com os resultados do teste de acompanhamento de referência com sinais do ensaio 2 - Sistema desacoplado com controle LQR, com os sinais obtidos na simulação [(a), (c) e (e)] e no experimento [(b), (d) e (f)]

(a) Sinais $r(t)$ e $y(t) \mathrm{em}^{\circ}$

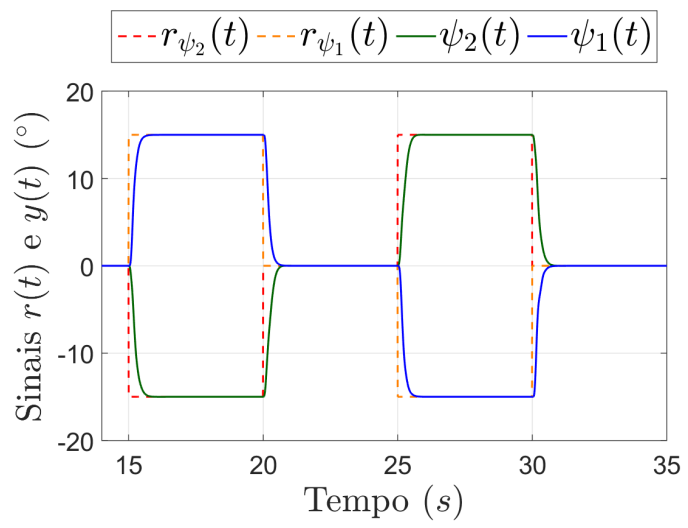

(c) Sinais de Controle $u(t)$ em N.m
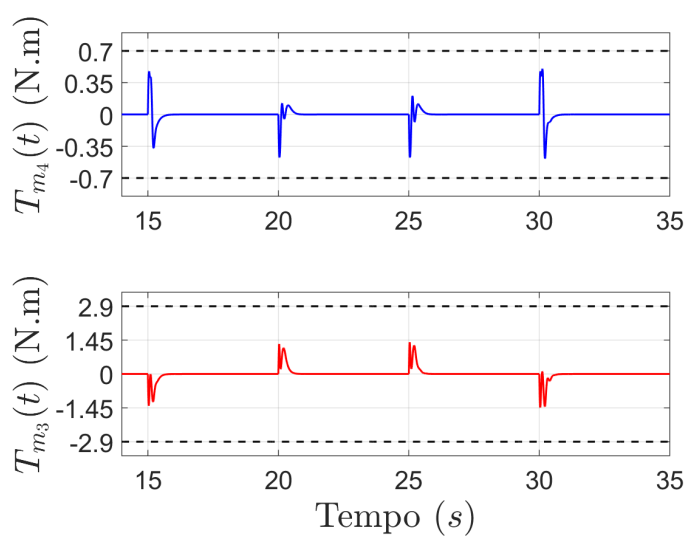

(e) Velocidades Angulares $\omega\left(k T_{s}\right)$ em $\% / \mathrm{s}$
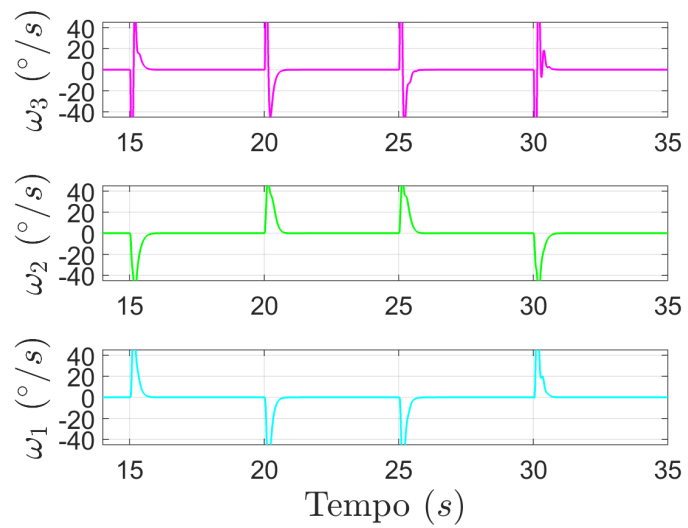

(b) Sinais $r(t)$ e $y(t) \mathrm{em}^{\circ}$

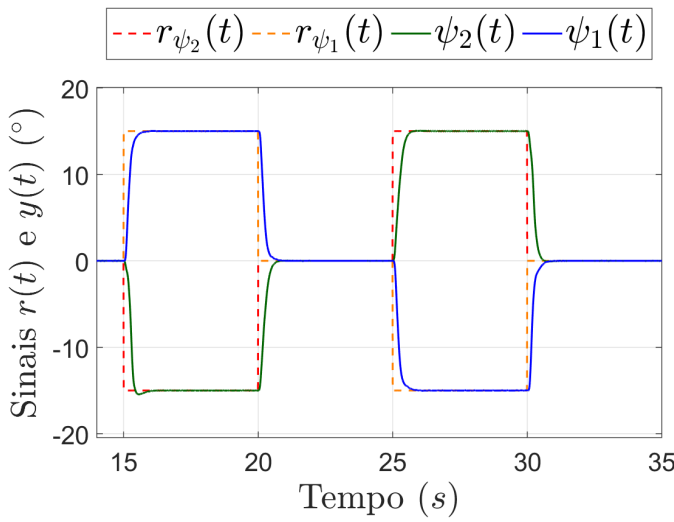

(d) Sinais de Controle $u(t)$ em N.m
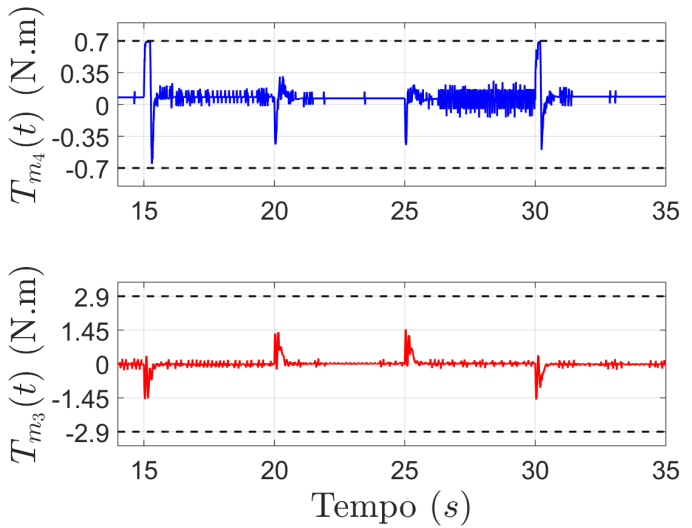

(f) Velocidades Angulares $\omega\left(k T_{s}\right) \mathrm{em} \% \mathrm{~s}$
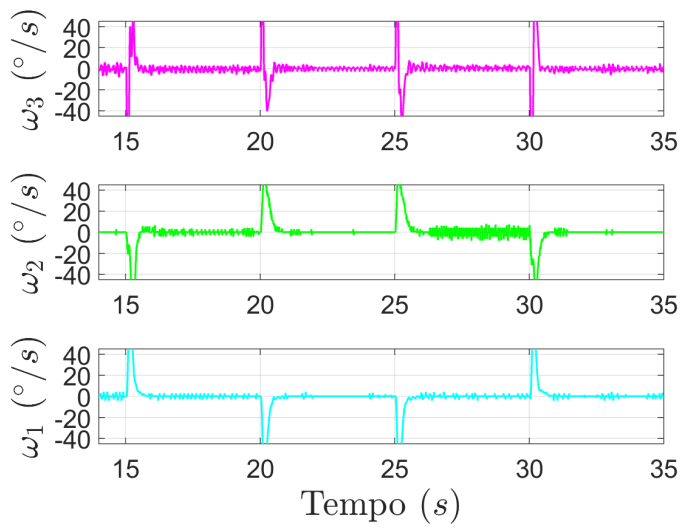

Fonte: Autor. 
Figura 41: Gráficos com os resultados do teste de rejeição à perturbação com sinais do ensaio 2 - Sistema desacoplado com controle LQR, com os sinais obtidos na simulação $[(\mathrm{a}),(\mathrm{c})$ e (e)] e no experimento [(b), (d) e (f)]

(a) Sinais $r(t)$ e $y(t) \mathrm{em}^{\circ}$

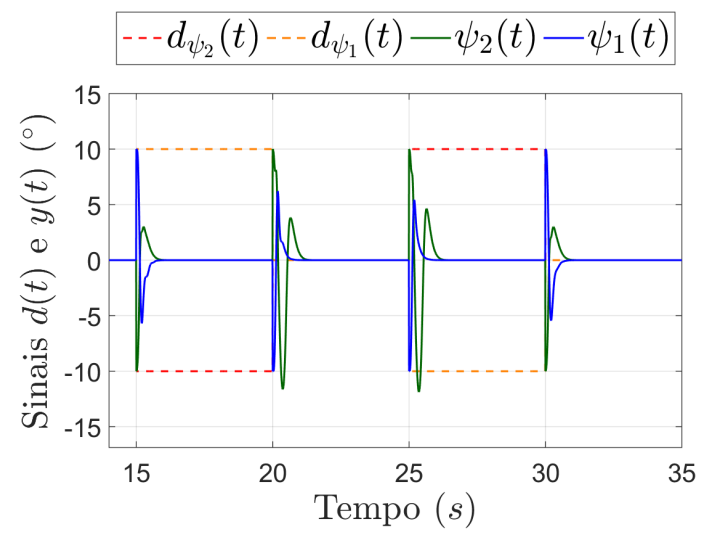

(c) Sinais de Controle $u(t)$ em N.m
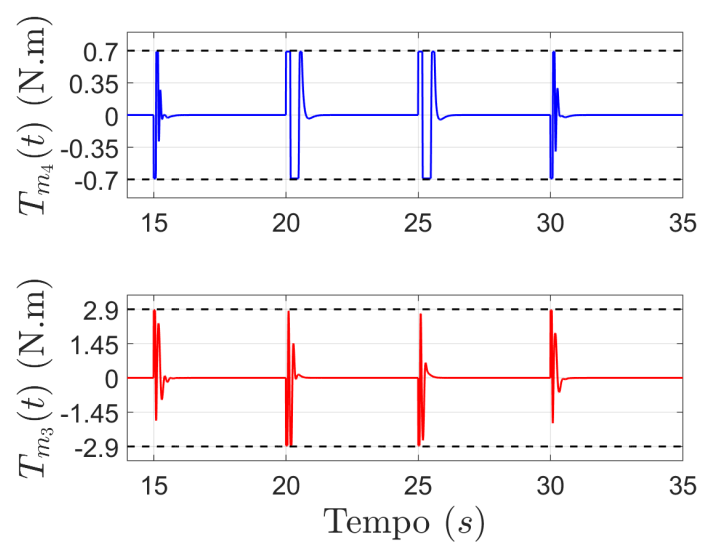

(e) Velocidades Angulares $\omega\left(k T_{s}\right) \mathrm{em} \% \mathrm{~s}$
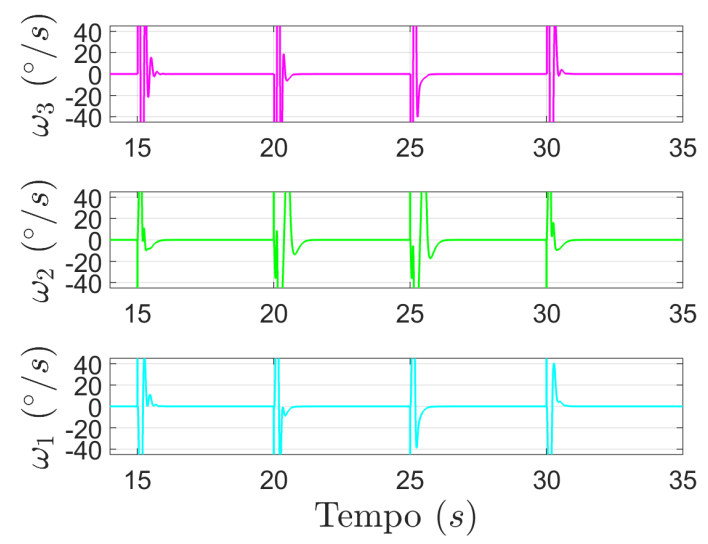

(b) Sinais $r(t)$ e $y(t) \mathrm{em}^{\circ}$

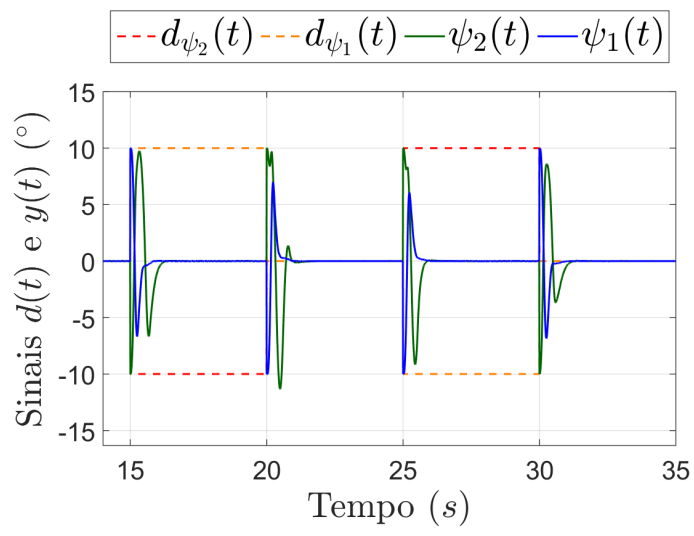

(d) Sinais de Controle $u(t)$ em N.m
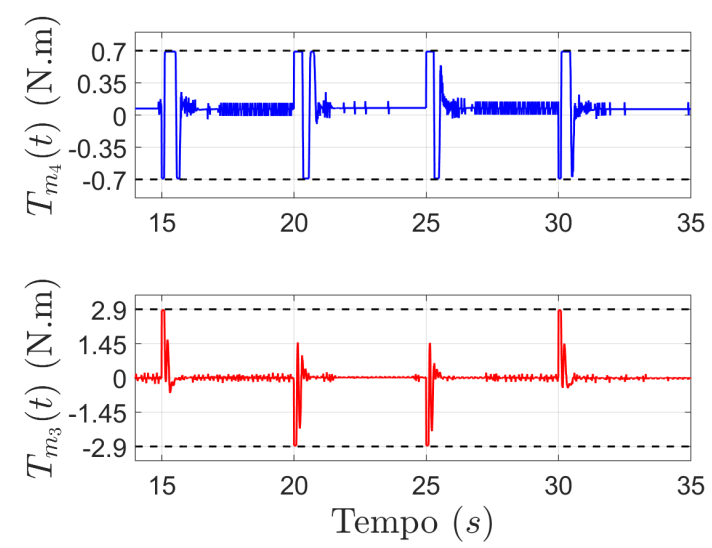

(f) Velocidades Angulares $\omega\left(k T_{s}\right) \mathrm{em} \% \mathrm{~s}$
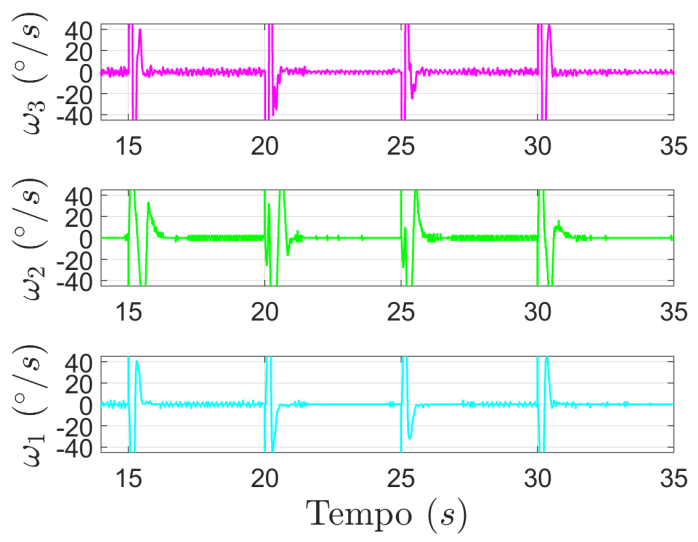

Fonte: Autor. 


\section{H.1.2.3 Ensaio 3 - Sinais Senoidais}

As Figuras 42 e 43 apresentam os resultados obtidos nos testes de acompanhamento de referência e de rejeição à perturbação, respectivamente.

Figura 42: Gráficos com os resultados do teste de acompanhamento de referência com sinais do ensaio 3 - Sistema desacoplado com controle LQR, com os sinais obtidos na simulação [(a), (c) e (e)] e no experimento [(b), (d) e (f)]

(a) Sinais $r(t)$ e $y(t) \mathrm{em}^{\circ}$

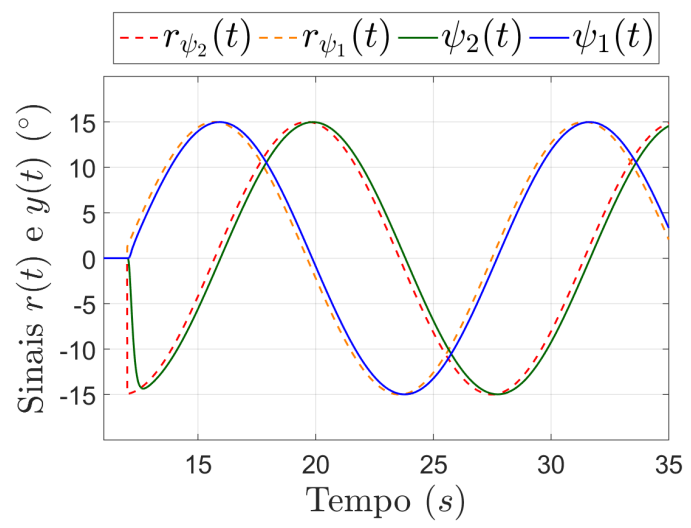

(c) Sinais de Controle $u(t)$ em N.m
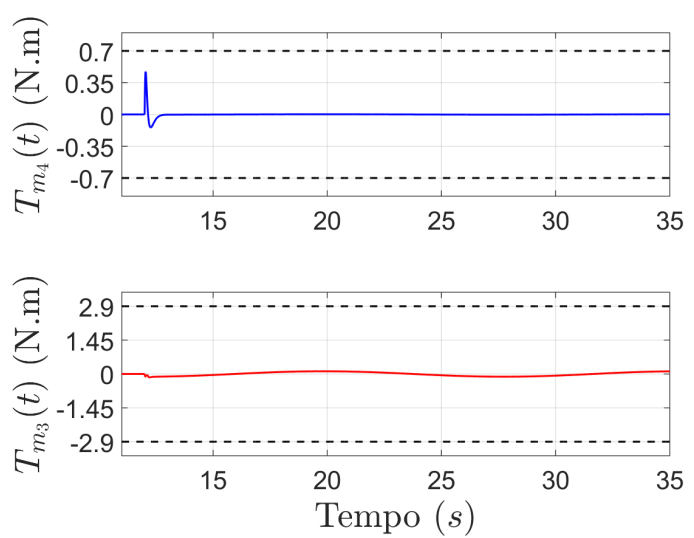

(e) Velocidades Angulares $\omega\left(k T_{s}\right) \mathrm{em} \% \mathrm{~s}$
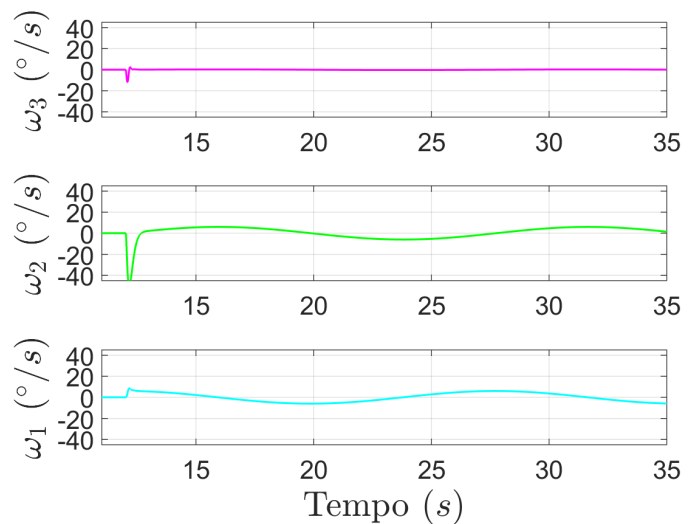

(b) Sinais $r(t)$ e $y(t) \mathrm{em}^{\circ}$

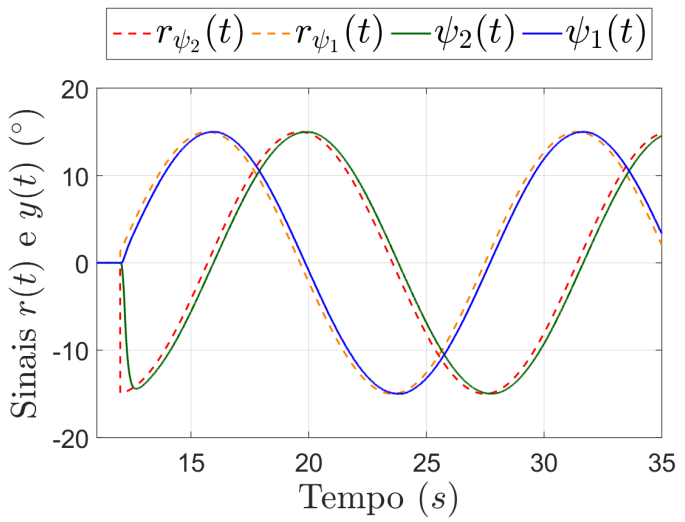

(d) Sinais de Controle $u(t)$ em N.m
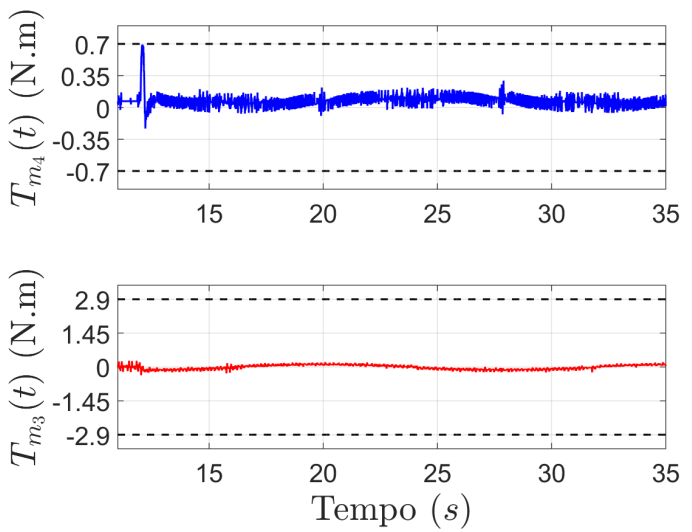

(f) Velocidades Angulares $\omega\left(k T_{s}\right)$ em \% $/ \mathrm{s}$
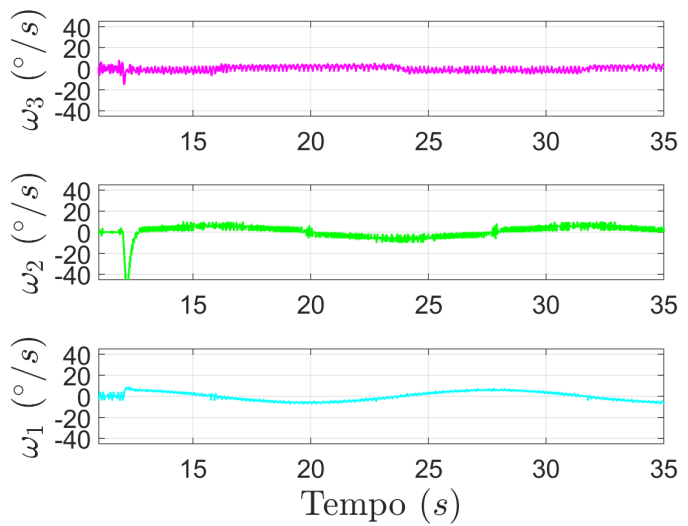

Fonte: Autor. 
Figura 43: Gráficos com os resultados do teste de rejeição à perturbação com sinais do ensaio 3 - Sistema desacoplado com controle LQR, com os sinais obtidos na simulação $[(\mathrm{a}),(\mathrm{c})$ e (e)] e no experimento [(b), (d) e (f)]

(a) Sinais $r(t)$ e $y(t) \mathrm{em}^{\circ}$

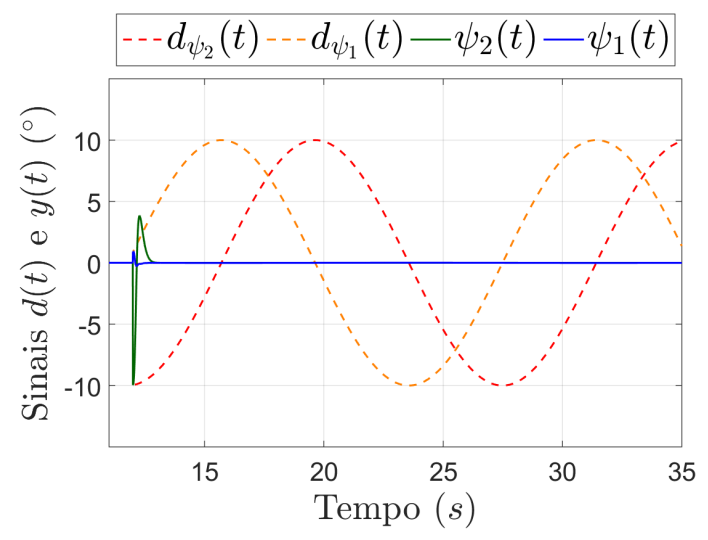

(c) Sinais de Controle $u(t)$ em N.m
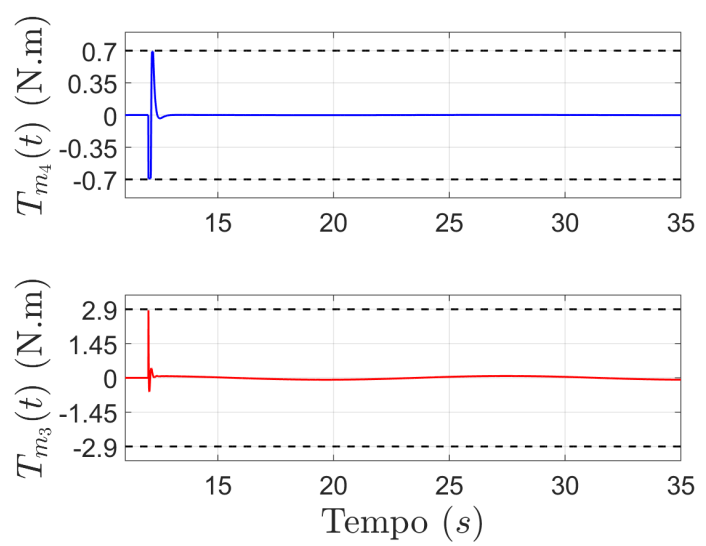

(e) Velocidades Angulares $\omega\left(k T_{s}\right) \mathrm{em} \% \mathrm{~s}$
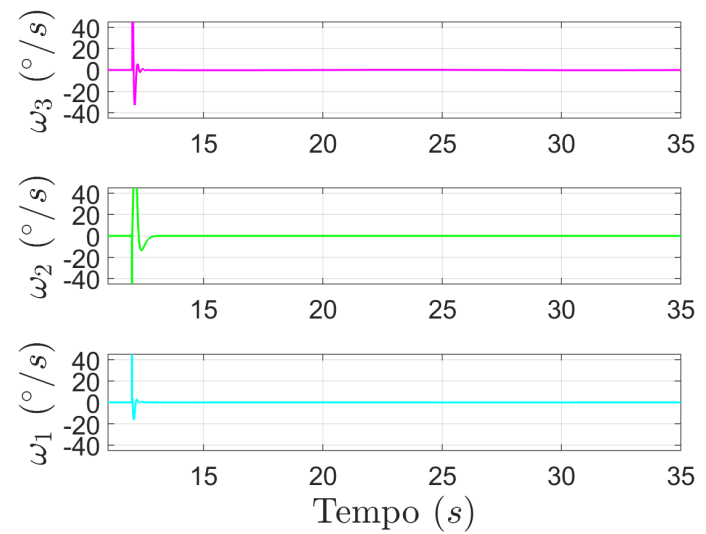

(b) Sinais $r(t)$ e $y(t) \mathrm{em}^{\circ}$

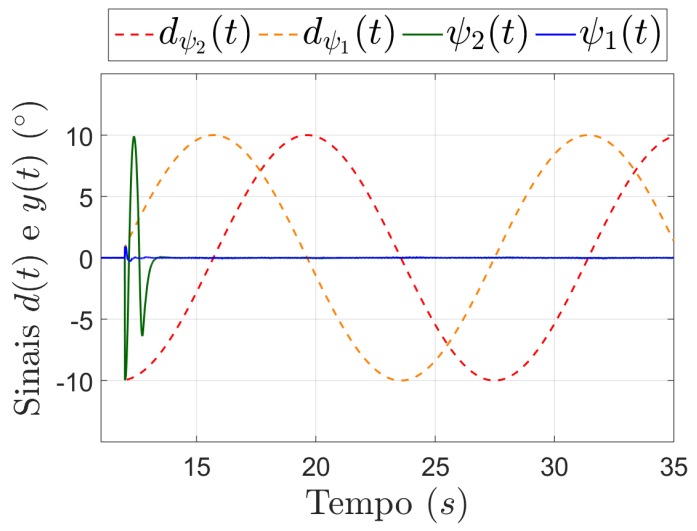

(d) Sinais de Controle $u(t)$ em N.m
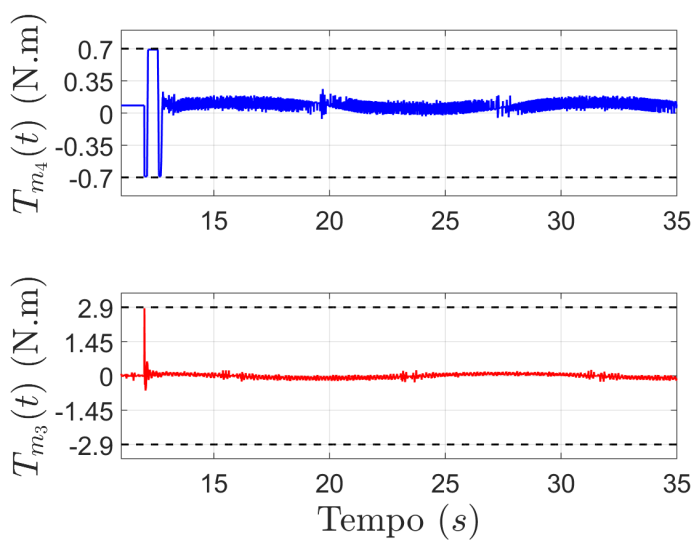

(f) Velocidades Angulares $\omega\left(k T_{s}\right) \mathrm{em} \% \mathrm{~s}$
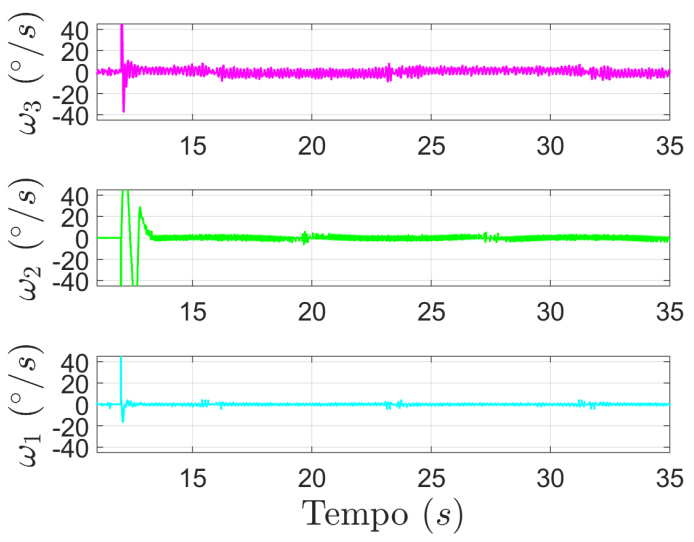

Fonte: Autor. 


\section{H.2 Caso Acoplado de Fase Mínima}

Para o sistema acoplado de fase mínima realiza-se o procedimento experimental, para cada caso de sinais considerados (ensaios 1, 2 e 3), com os sistemas contendo a planta na configuração acoplada de fase mínima (no ponto de operação $\psi_{2_{0}}=-20^{\circ}, \psi_{3_{0}}=20^{\circ}$ e $\Omega=400 R P M)$ e os controladores digitais projetados.

\section{H.2.1 Sistema com o Controlador PID}

Para o sistema acoplado de fase mínima, o controlador digital PID utilizado é projetado em relação ao modelo desacoplado da planta. Isso se deve ao fato do controlador PID projetado em relação ao modelo acoplado de fase mínima ter apresentado resultados piores em comparação ao projetado em relação ao modelo desacoplado, como informado e justificado na Subseção 4.1.2. Os resultados obtidos para os 3 ensaios de sinais considerados são apresentados a seguir.

\section{H.2.1.1 Ensaio 1 - Pulsos Retangulares Separados}

As Figuras 44 e 45 apresentam os resultados obtidos no teste de acompanhamento de referência com amplitudes positivas e negativas, respectivamente. e as Figuras 46 e 47 os resultados obtidos no teste de rejeição à perturbação com amplitudes positivas e negativas, respectivamente. 
Figura 44: Gráficos com os resultados do teste de acompanhamento de referência com sinais do ensaio 1 (positivo) - Sistema acoplado de fase mínima com controle PID, com os sinais obtidos na simulação [(a), (c) e (e)] e no experimento [(b), (d) e (f)]

(a) Sinais $r(t)$ e $y(t)$ em $^{\circ}$

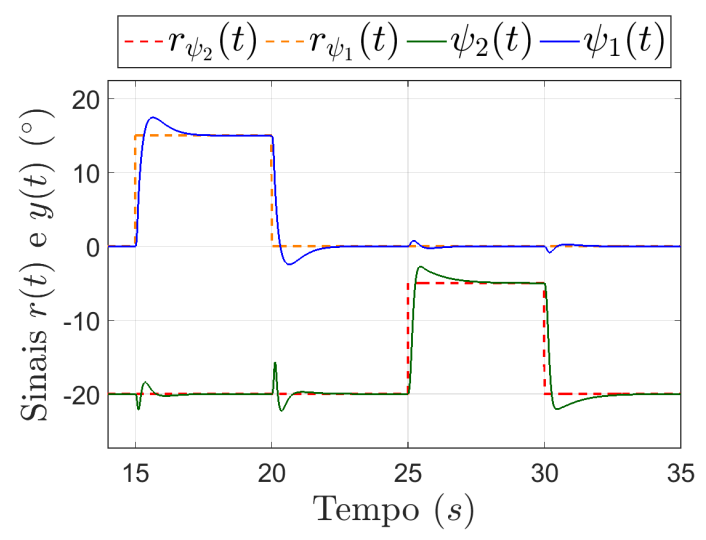

(c) Sinais de Controle $u(t)$ em N.m
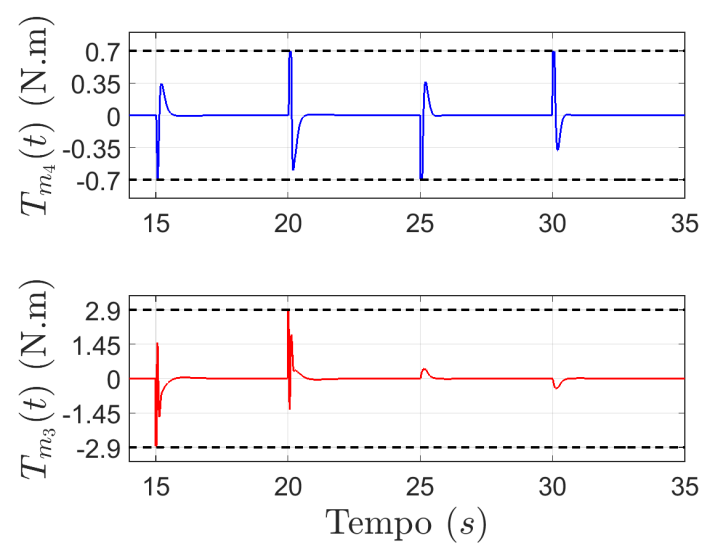

(e) Velocidades Angulares $\omega\left(k T_{s}\right)$ em $\% / \mathrm{s}$
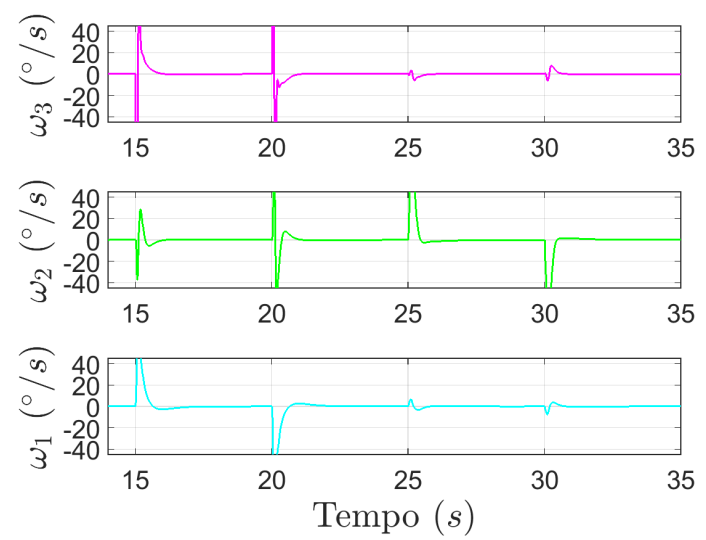

(b) Sinais $r(t)$ e $y(t) \mathrm{em}^{\circ}$

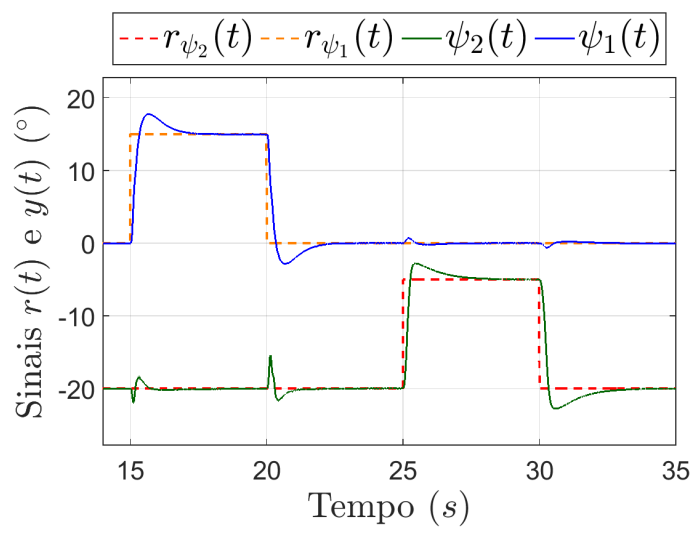

(d) Sinais de Controle $u(t)$ em N.m
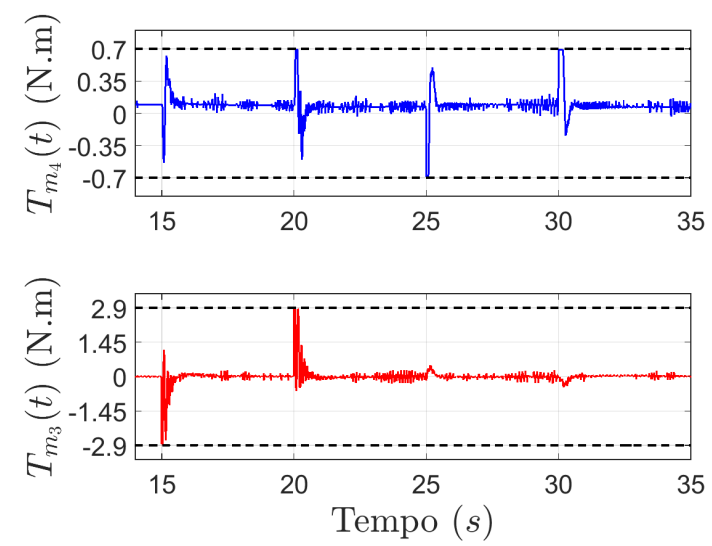

(f) Velocidades Angulares $\omega\left(k T_{s}\right)$ em $\% / \mathrm{s}$
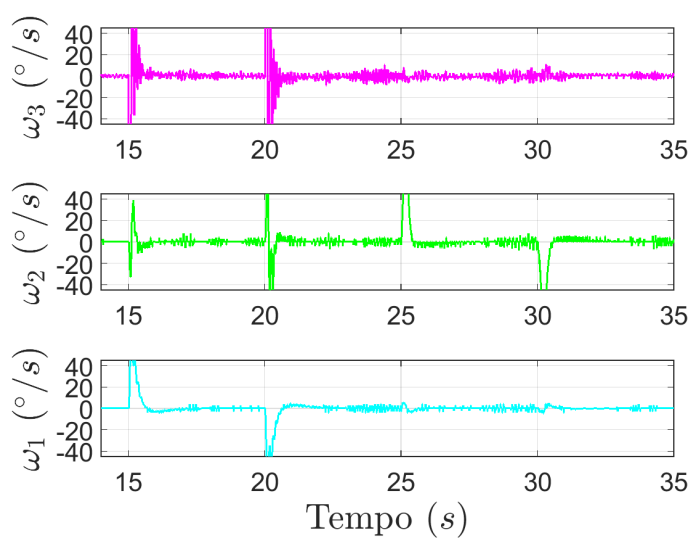

Fonte: Autor. 
Figura 45: Gráficos com os resultados do teste de acompanhamento de referência com sinais do ensaio 1 (negativo) - Sistema acoplado de fase mínima com controle PID, com os sinais obtidos na simulação [(a), (c) e (e)] e no experimento [(b), (d) e (f)]

(a) Sinais $r(t)$ e $y(t)$ em $^{\circ}$

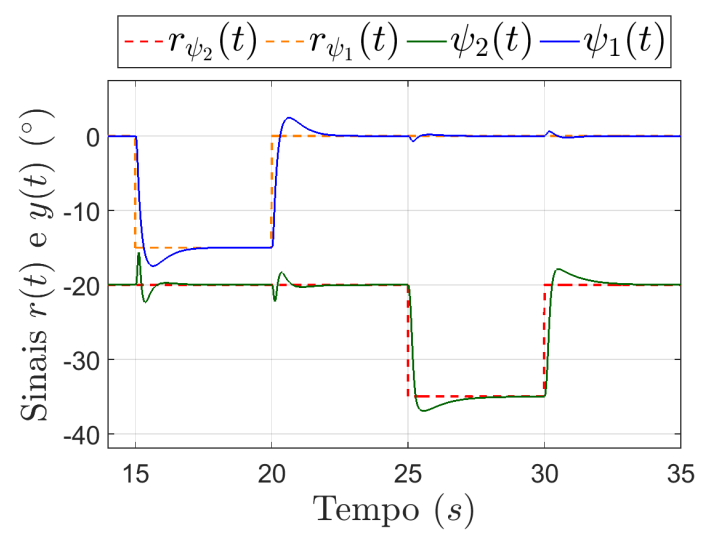

(c) Sinais de Controle $u(t)$ em N.m
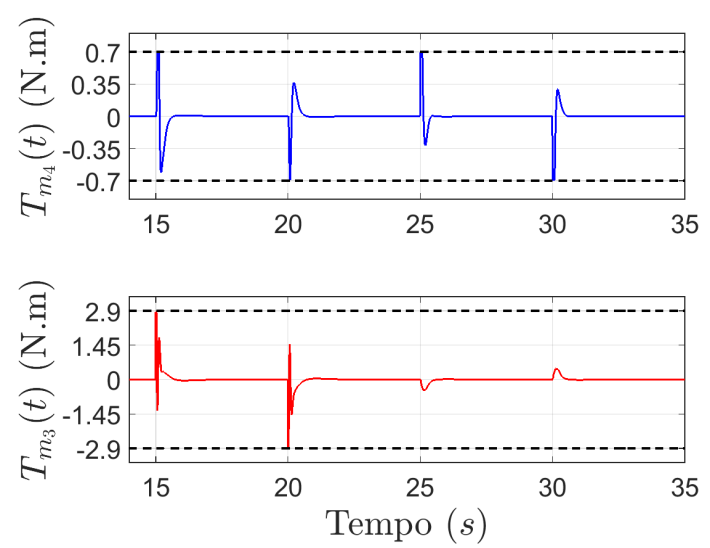

(e) Velocidades Angulares $\omega\left(k T_{s}\right)$ em $\% / \mathrm{s}$
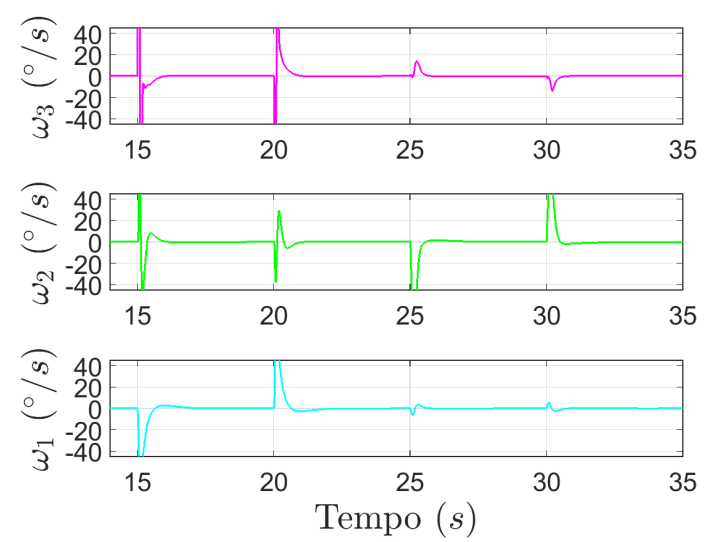

(b) Sinais $r(t)$ e $y(t) \mathrm{em}^{\circ}$

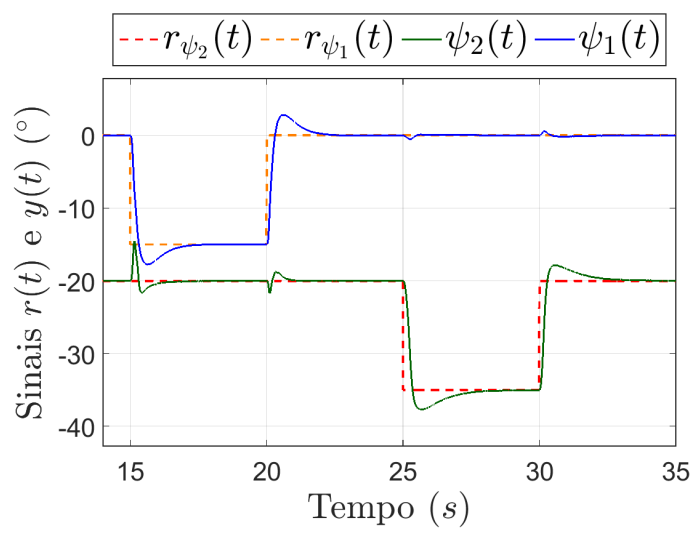

(d) Sinais de Controle $u(t)$ em N.m
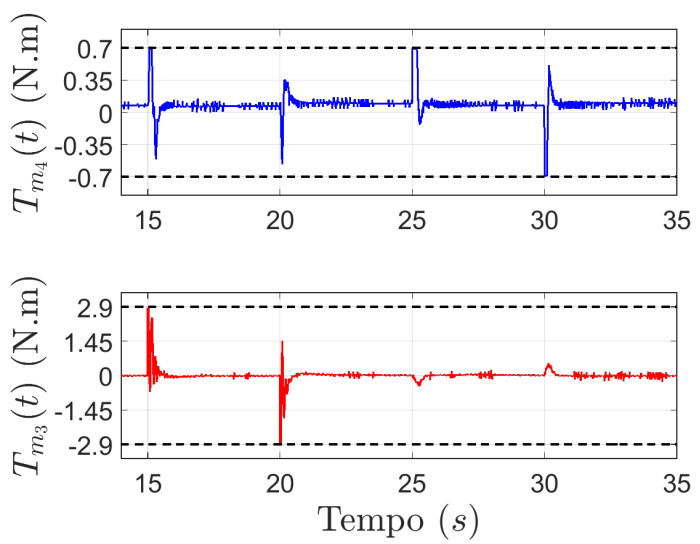

(f) Velocidades Angulares $\omega\left(k T_{s}\right)$ em $\% / \mathrm{s}$
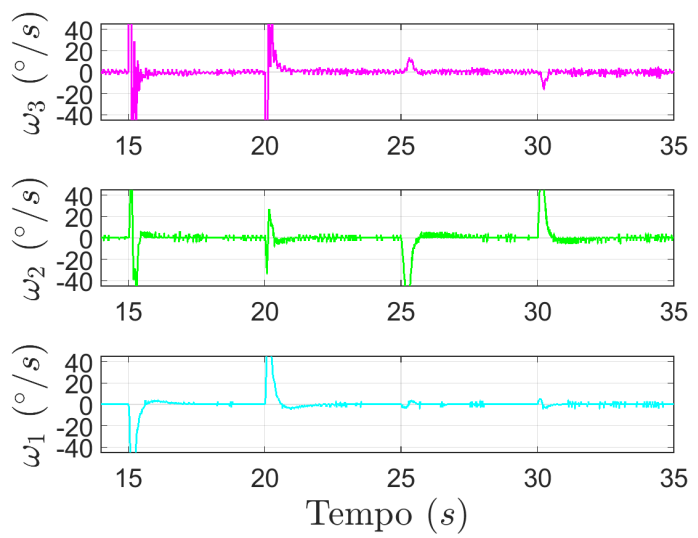

Fonte: Autor. 
Figura 46: Gráficos com os resultados do teste de rejeição à perturbação com sinais do ensaio 1 (positivo) - Sistema acoplado de fase mínima com controle PID, com os sinais obtidos na simulação [(a), (c) e (e)] e no experimento [(b), (d) e (f)]

(a) Sinais $r(t)$ e $y(t)$ em $^{\circ}$

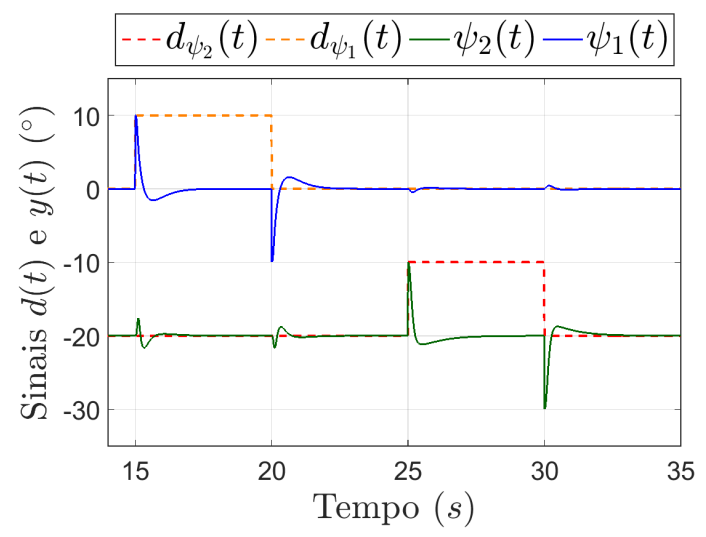

(c) Sinais de Controle $u(t)$ em N.m
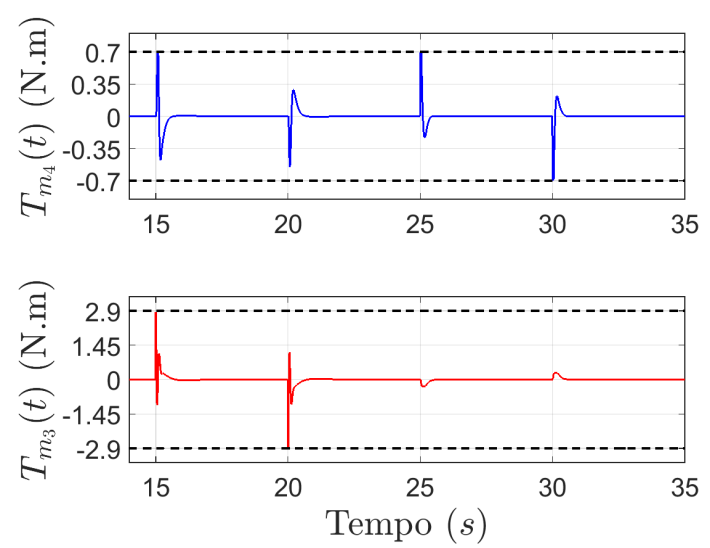

(e) Velocidades Angulares $\omega\left(k T_{s}\right)$ em $\% / \mathrm{s}$
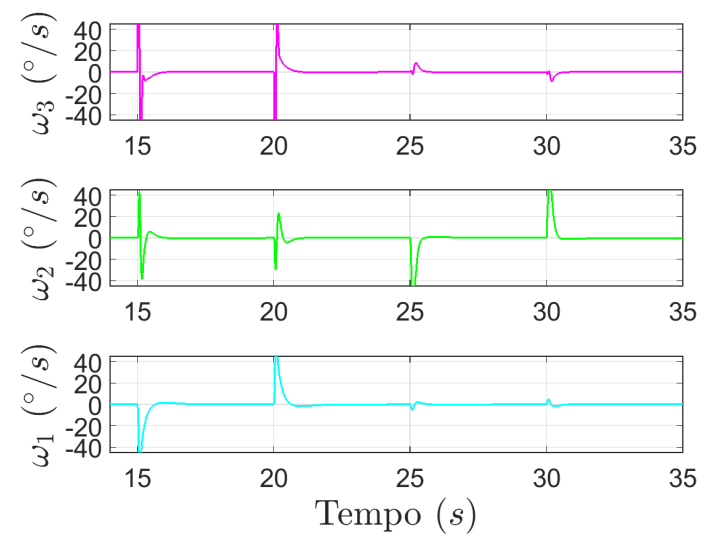

(b) Sinais $r(t)$ e $y(t) \mathrm{em}^{\circ}$

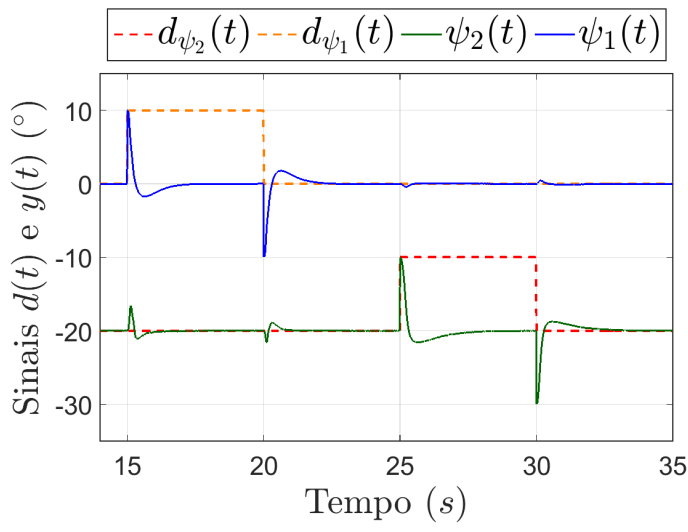

(d) Sinais de Controle $u(t)$ em N.m
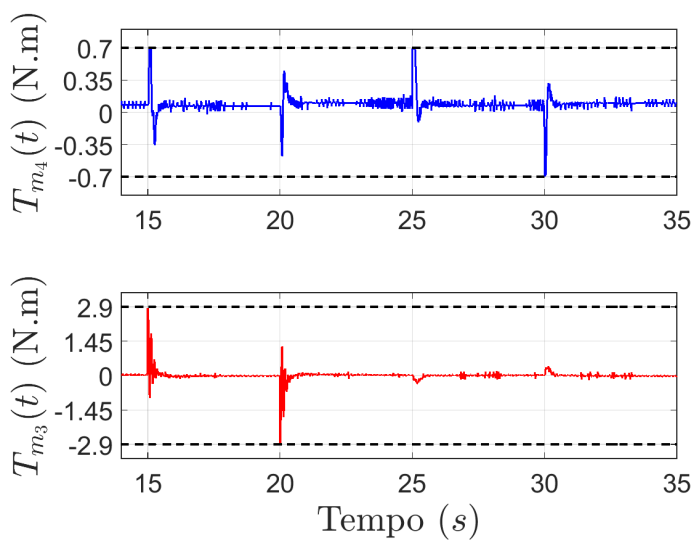

(f) Velocidades Angulares $\omega\left(k T_{s}\right)$ em $\% / \mathrm{s}$
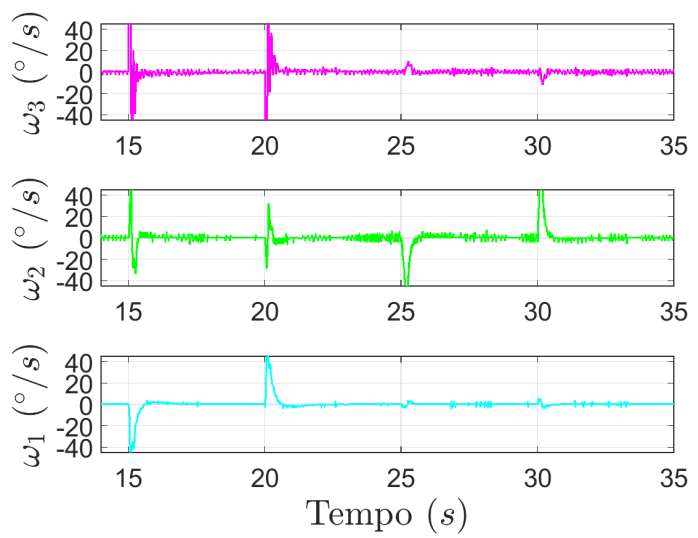

Fonte: Autor. 
Figura 47: Gráficos com os resultados do teste de rejeição à perturbação com sinais do ensaio 1 (negativo) - Sistema acoplado de fase mínima com controle PID, com os sinais obtidos na simulação [(a), (c) e (e)] e no experimento [(b), (d) e (f)]

(a) Sinais $r(t)$ e $y(t)$ em $^{\circ}$

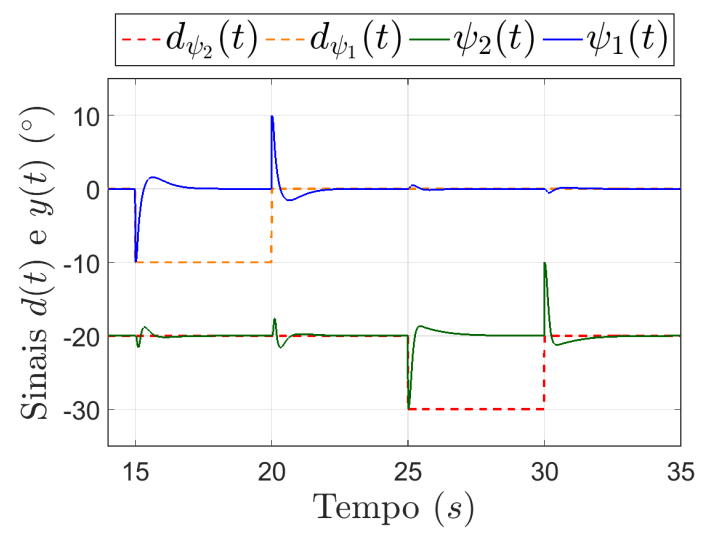

(c) Sinais de Controle $u(t)$ em N.m
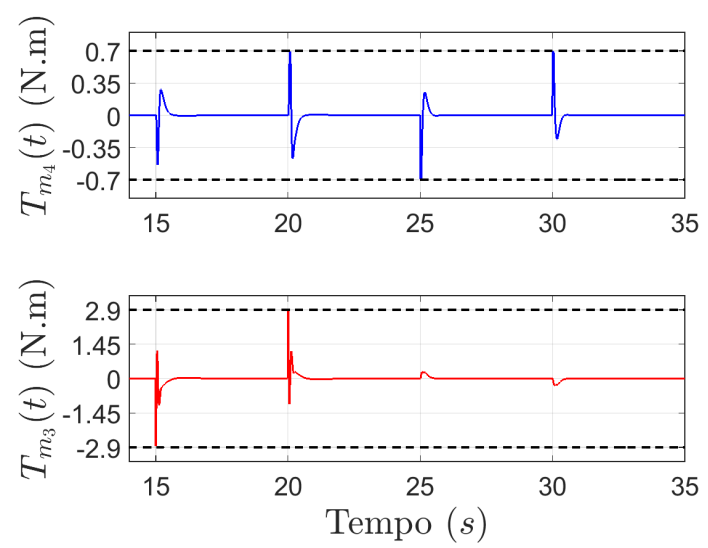

(e) Velocidades Angulares $\omega\left(k T_{s}\right)$ em $\% / \mathrm{s}$
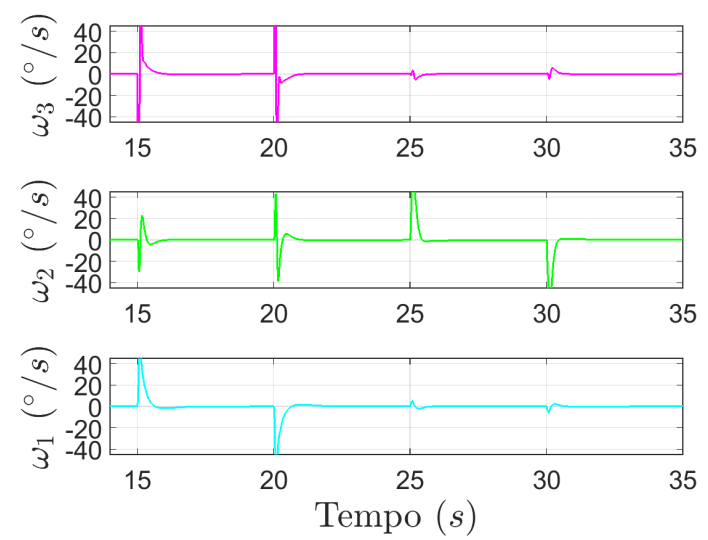

(b) Sinais $r(t)$ e $y(t)$ em $^{\circ}$

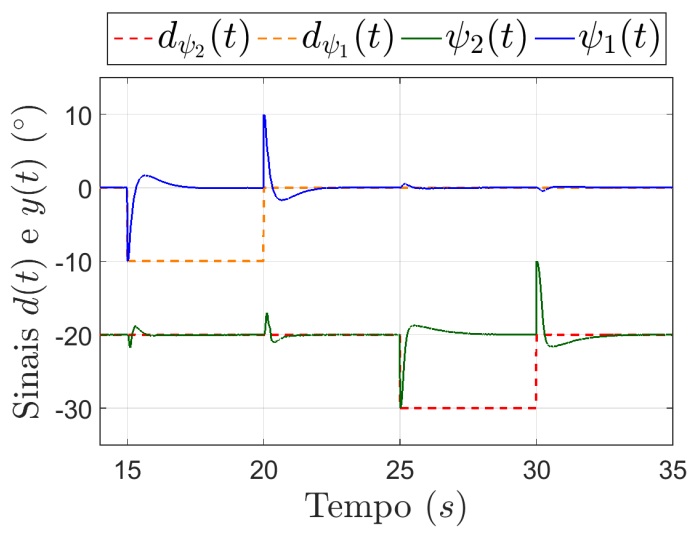

(d) Sinais de Controle $u(t)$ em N.m
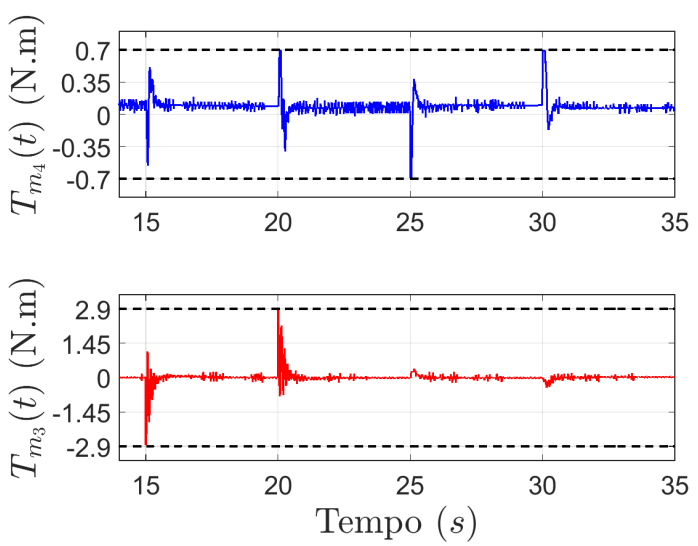

(f) Velocidades Angulares $\omega\left(k T_{s}\right)$ em $\% / \mathrm{s}$
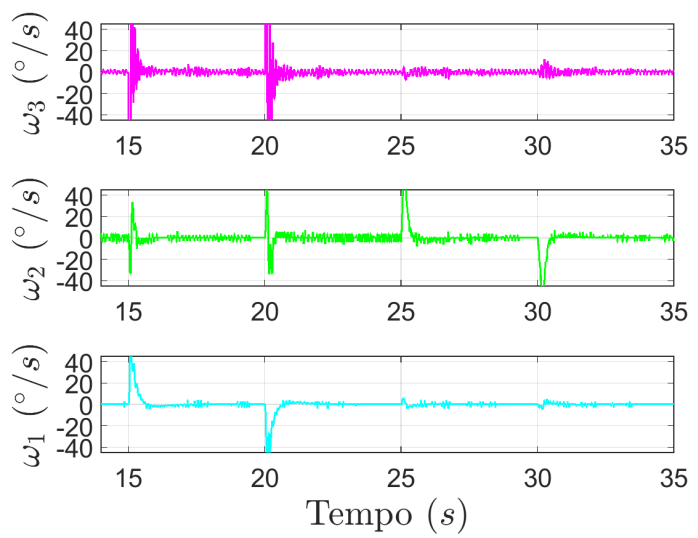

Fonte: Autor. 


\section{H.2.1.2 Ensaio 2 - Pulsos Retangulares Simultâneos}

As Figuras 48 e 49 apresentam os resultados obtidos nos testes de acompanhamento de referência e de rejeição à perturbação, respectivamente.

Figura 48: Gráficos com os resultados do teste de acompanhamento de referência com sinais do ensaio 2 - Sistema acoplado de fase mínima com controle PID, com os sinais obtidos na simulação [(a), (c) e (e)] e no experimento [(b), (d) e (f)]

(a) Sinais $r(t)$ e $y(t) \mathrm{em}^{\circ}$

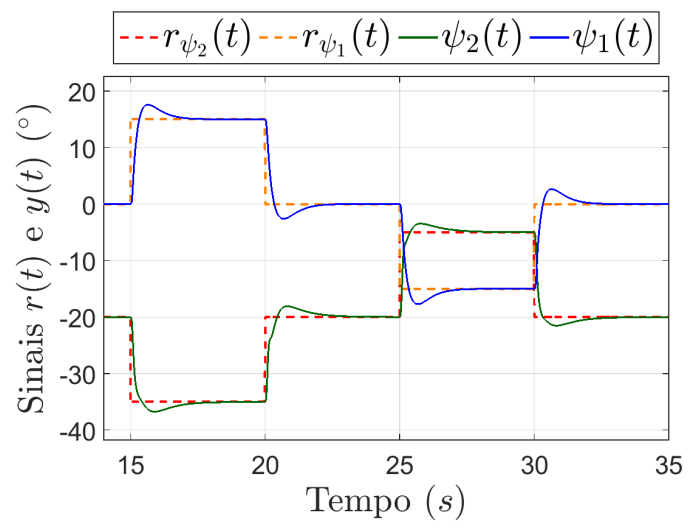

(c) Sinais de Controle $u(t)$ em N.m
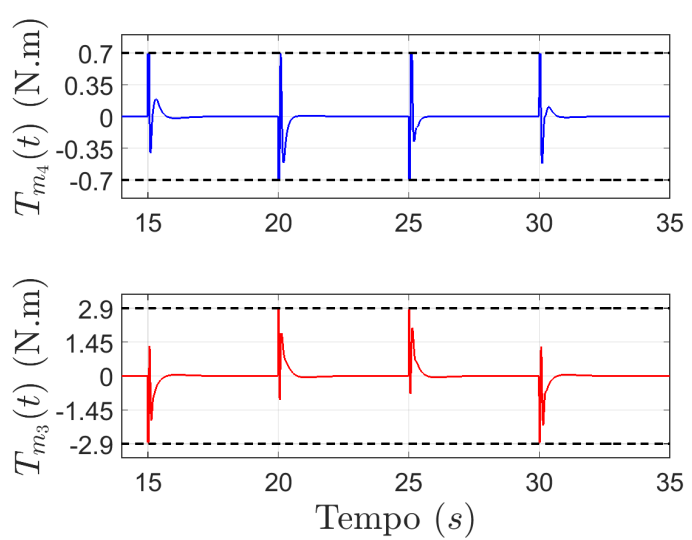

(e) Velocidades Angulares $\omega\left(k T_{s}\right) \mathrm{em} \% \mathrm{~s}$
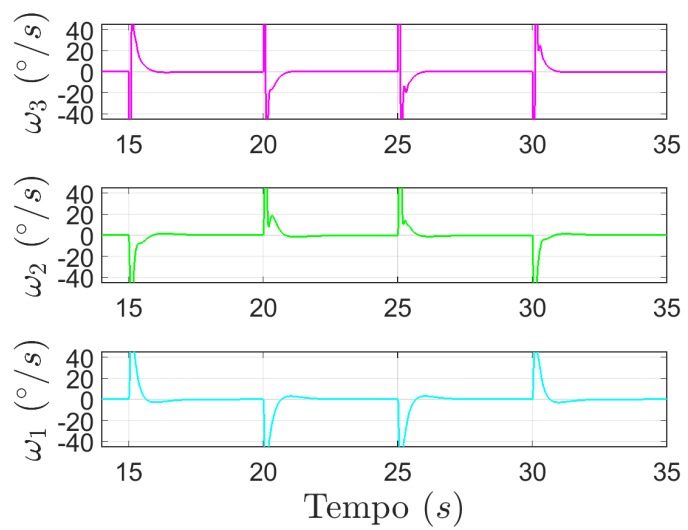

(b) Sinais $r(t)$ e $y(t) \mathrm{em}^{\circ}$

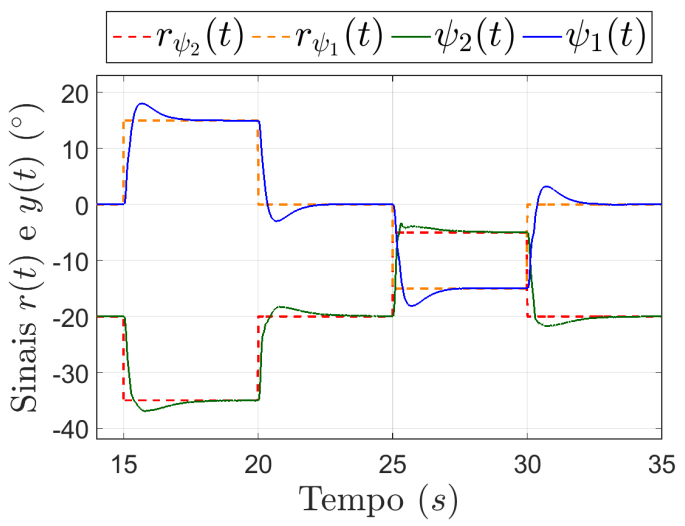

(d) Sinais de Controle $u(t)$ em N.m
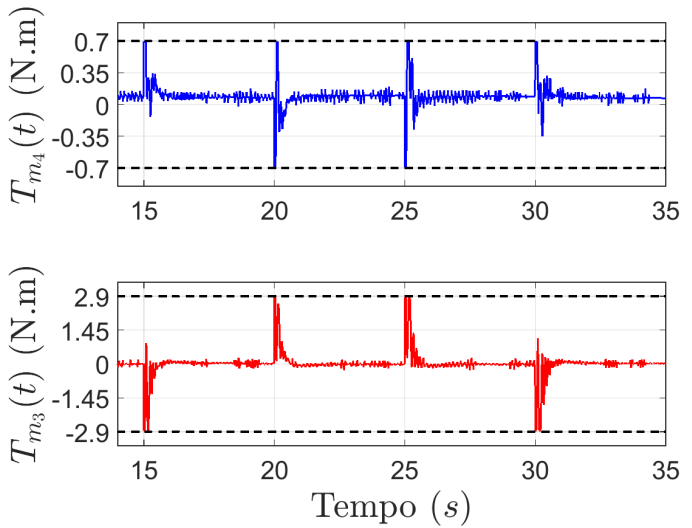

(f) Velocidades Angulares $\omega\left(k T_{s}\right)$ em \% $/ \mathrm{s}$
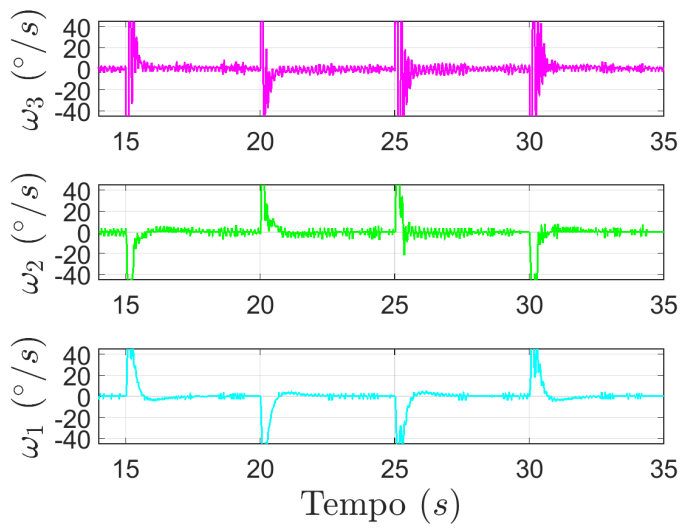

Fonte: Autor. 
Figura 49: Gráficos com os resultados do teste de rejeição à perturbação com sinais do ensaio 2 - Sistema acoplado de fase mínima com controle PID, com os sinais obtidos na simulação [(a), (c) e (e)] e no experimento [(b), (d) e (f)]
(a) Sinais $r(t)$ e $y(t)$ em $^{\circ}$
(b) Sinais $r(t)$ e $y(t) \mathrm{em}^{\circ}$

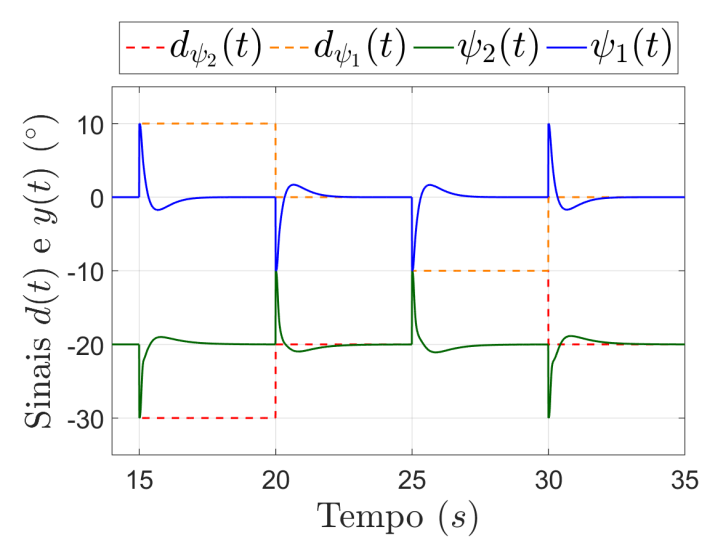

(c) Sinais de Controle $u(t)$ em N.m
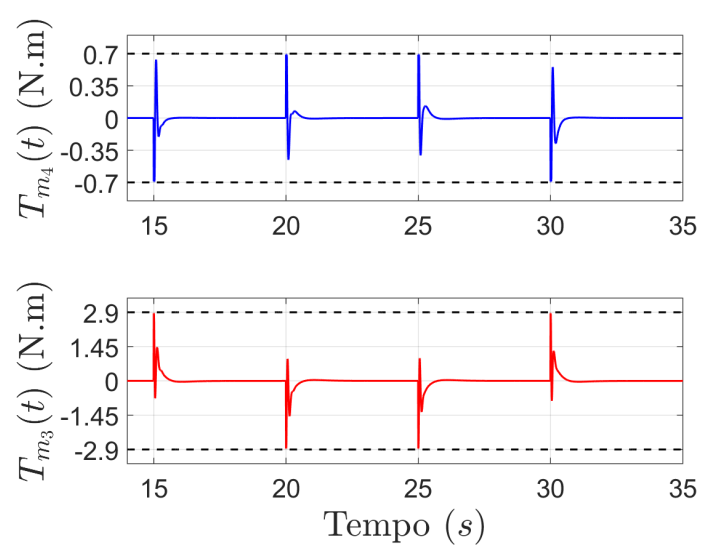

(e) Velocidades Angulares $\omega\left(k T_{s}\right) \mathrm{em} \% \mathrm{~s}$
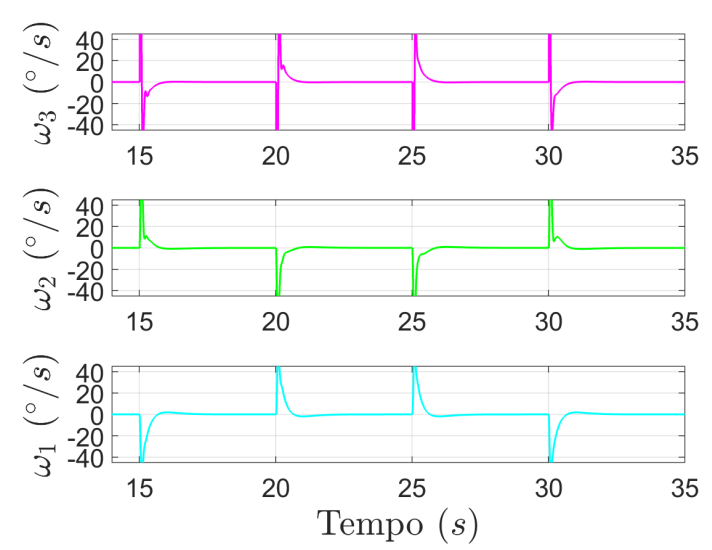

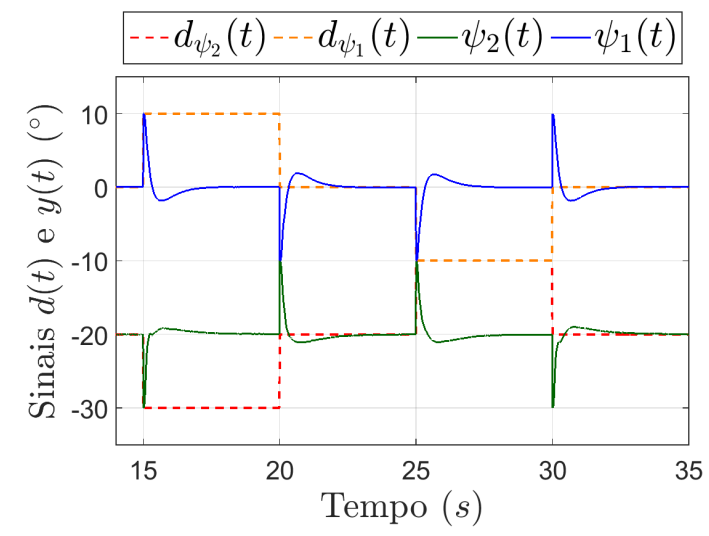

(d) Sinais de Controle $u(t)$ em N.m
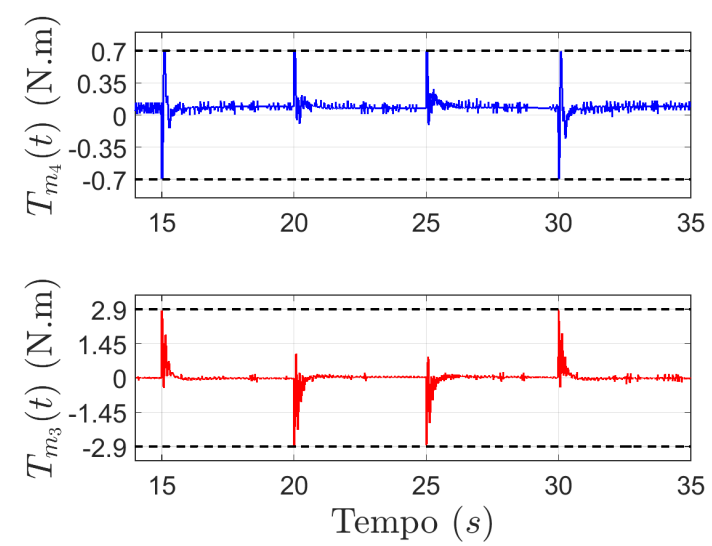

(f) Velocidades Angulares $\omega\left(k T_{s}\right) \mathrm{em} \% \mathrm{~s}$
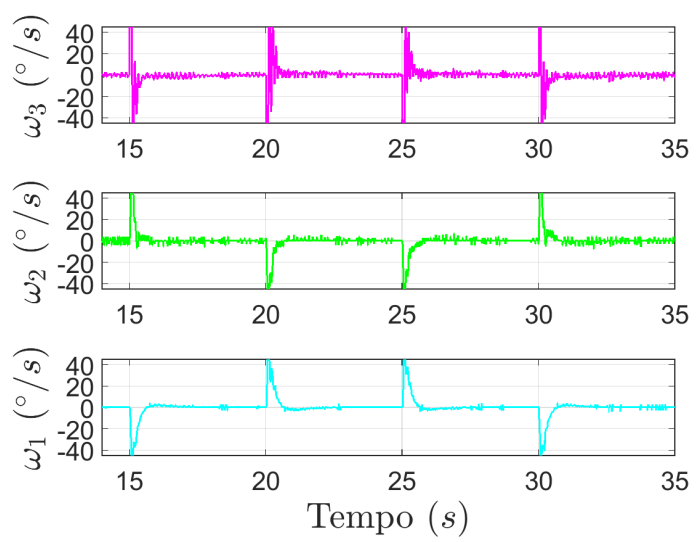

Fonte: Autor. 


\section{H.2.1.3 Ensaio 3 - Sinais Senoidais}

As Figuras 50 e 51 apresentam os resultados obtidos nos testes de acompanhamento de referência e de rejeição à perturbação, respectivamente.

Figura 50: Gráficos com os resultados do teste de acompanhamento de referência com sinais do ensaio 3 - Sistema acoplado de fase mínima com controle PID, com os sinais obtidos na simulação [(a), (c) e (e)] e no experimento [(b), (d) e (f)]

(a) Sinais $r(t)$ e $y(t) \mathrm{em}^{\circ}$

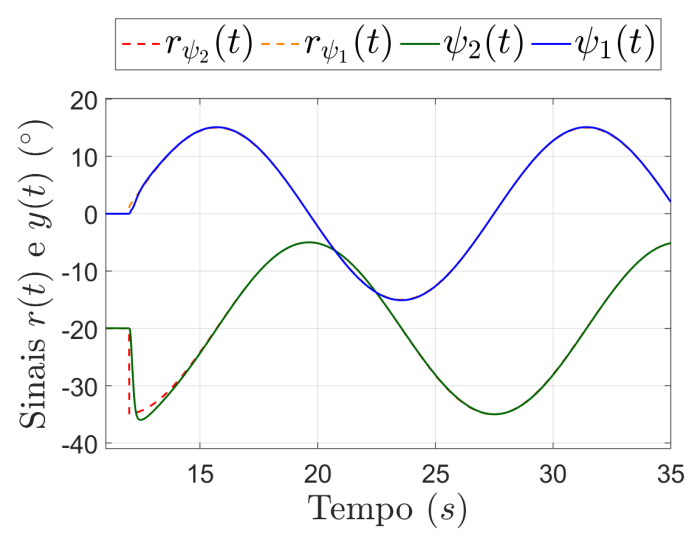

(c) Sinais de Controle $u(t)$ em N.m
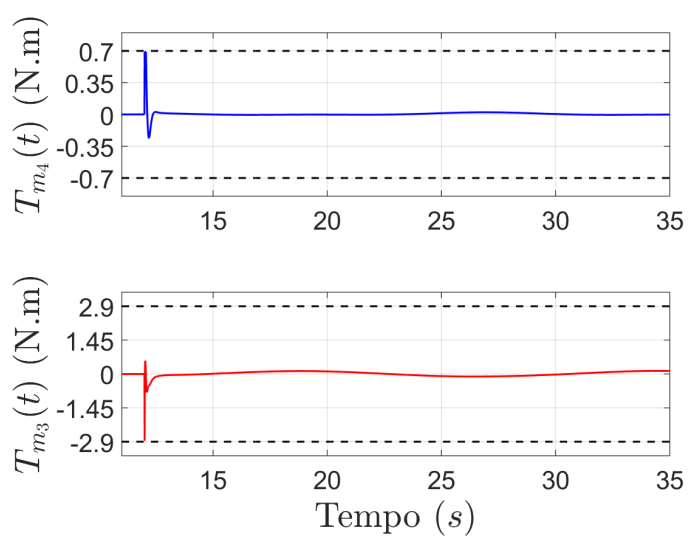

(e) Velocidades Angulares $\omega\left(k T_{s}\right) \mathrm{em} \% \mathrm{~s}$
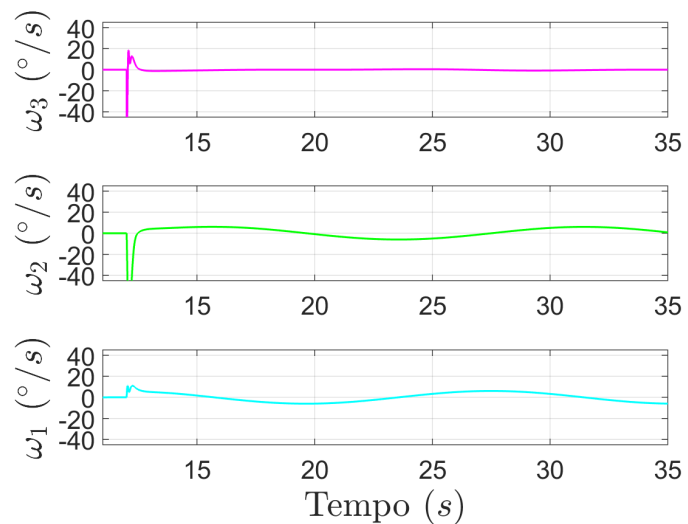

(b) Sinais $r(t)$ e $y(t) \mathrm{em}^{\circ}$

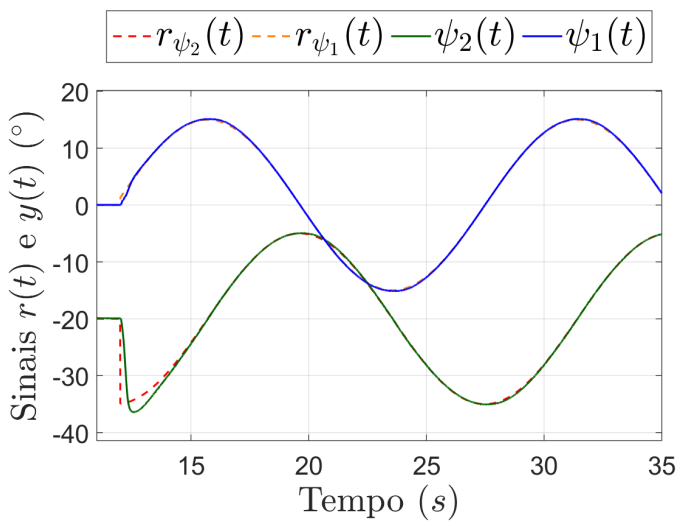

(d) Sinais de Controle $u(t)$ em N.m
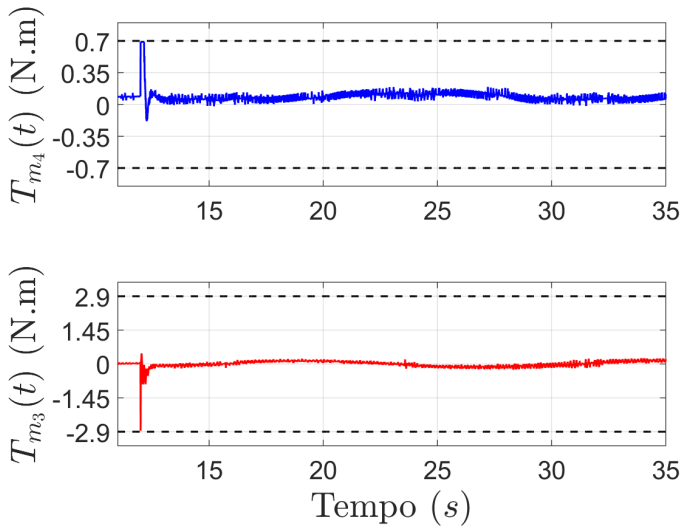

(f) Velocidades Angulares $\omega\left(k T_{s}\right)$ em \% $/ \mathrm{s}$
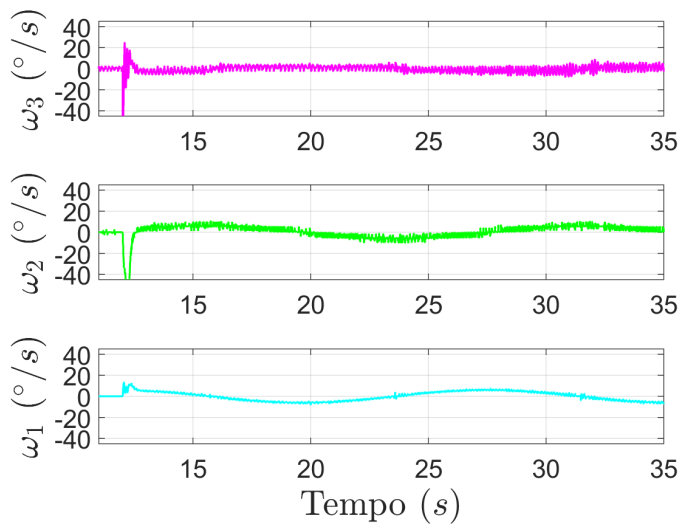

Fonte: Autor. 
Figura 51: Gráficos com os resultados do teste de rejeição à perturbação com sinais do ensaio 3 - Sistema acoplado de fase mínima com controle PID, com os sinais obtidos na simulação [(a), (c) e (e)] e no experimento [(b), (d) e (f)]

(a) Sinais $r(t)$ e $y(t) \mathrm{em}^{\circ}$

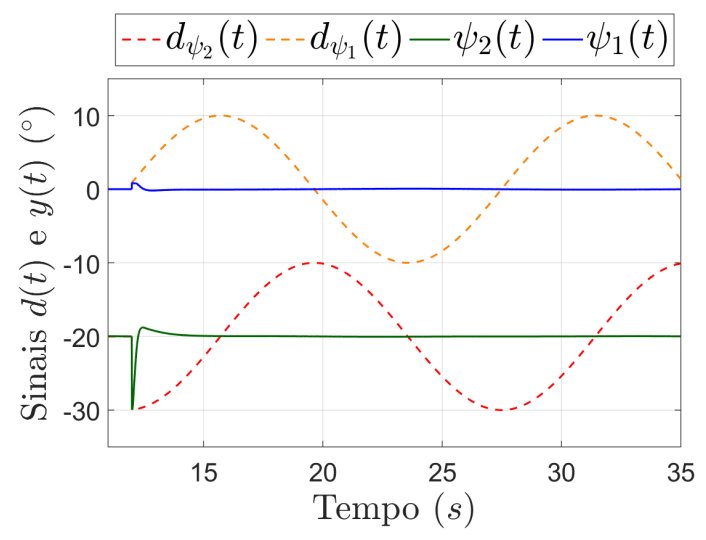

(c) Sinais de Controle $u(t)$ em N.m
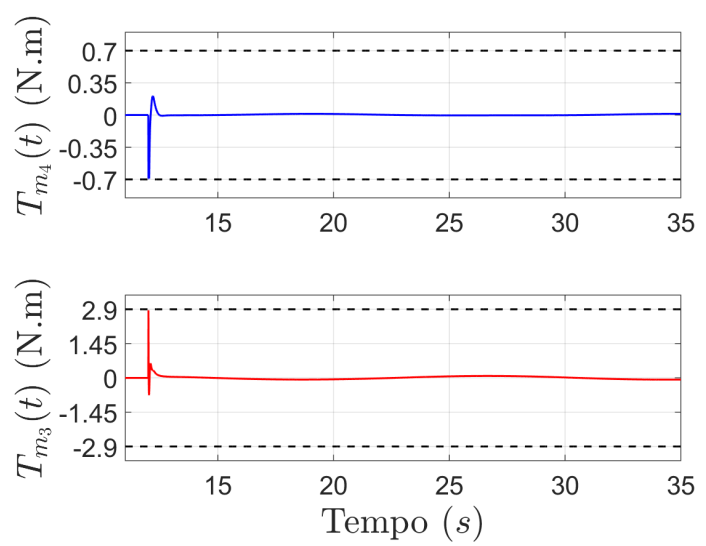

(e) Velocidades Angulares $\omega\left(k T_{s}\right) \mathrm{em} \% \mathrm{~s}$
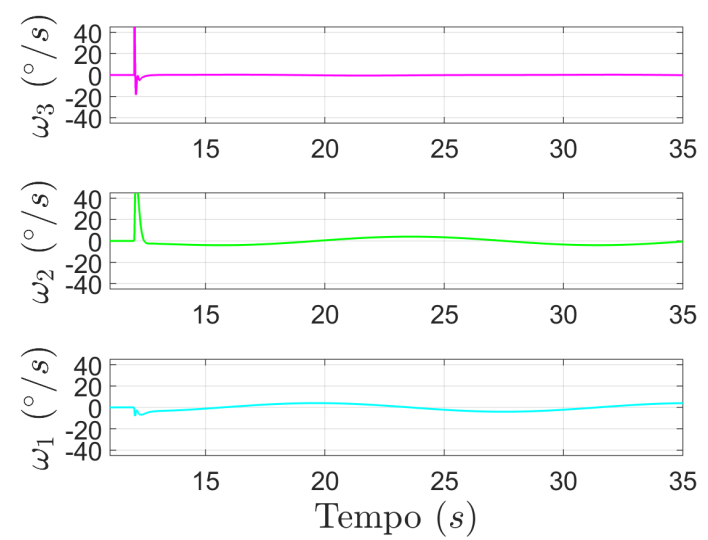

(b) Sinais $r(t)$ e $y(t) \mathrm{em}^{\circ}$

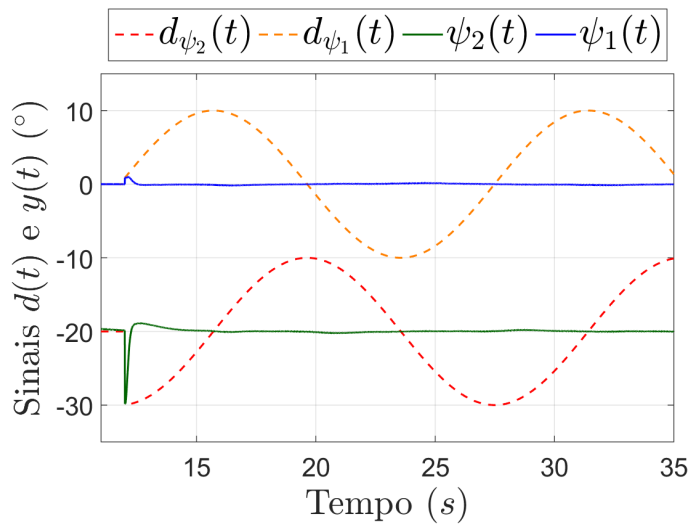

(d) Sinais de Controle $u(t)$ em N.m
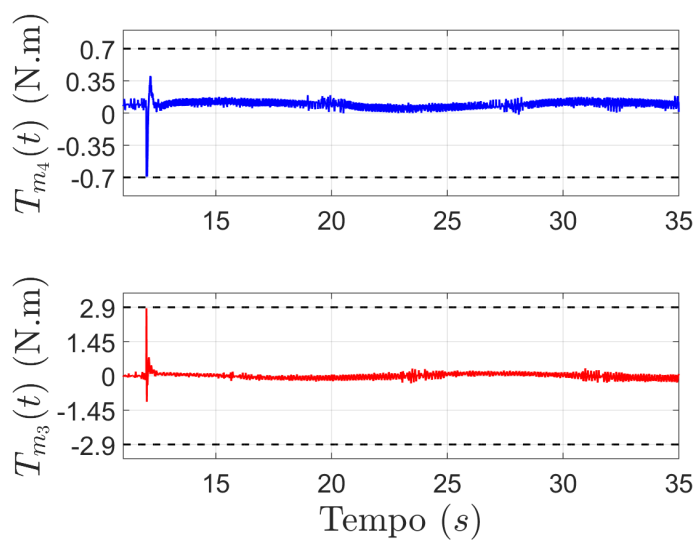

(f) Velocidades Angulares $\omega\left(k T_{s}\right) \mathrm{em} \% \mathrm{~s}$
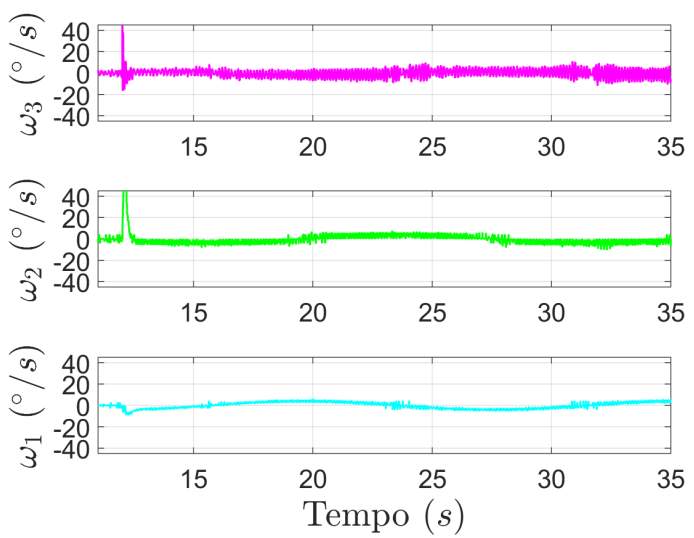

Fonte: Autor. 


\section{H.2.2 Sistema com o Controlador LQR}

Para o sistema acoplado de fase mínima, o controlador digital LQR utilizado é projetado em relação ao modelo acoplado de fase mínima da planta. Os resultados obtidos para os 3 ensaios de sinais considerados são apresentados a seguir.

\section{H.2.2.1 Ensaio 1 - Pulsos Retangulares Separados}

As Figuras 52 e 53 apresentam os resultados obtidos no teste de acompanhamento de referência com amplitudes positivas e negativas, respectivamente. e as Figuras 54 e 55 os resultados obtidos no teste de rejeição à perturbação com amplitudes positivas e negativas, respectivamente. 
Figura 52: Gráficos com os resultados do teste de acompanhamento de referência com sinais do ensaio 1 (positivo) - Sistema acoplado de fase mínima com controle LQR, com os sinais obtidos na simulação [(a), (c) e (e)] e no experimento [(b), (d) e (f)]

(a) Sinais $r(t)$ e $y(t)$ em $^{\circ}$

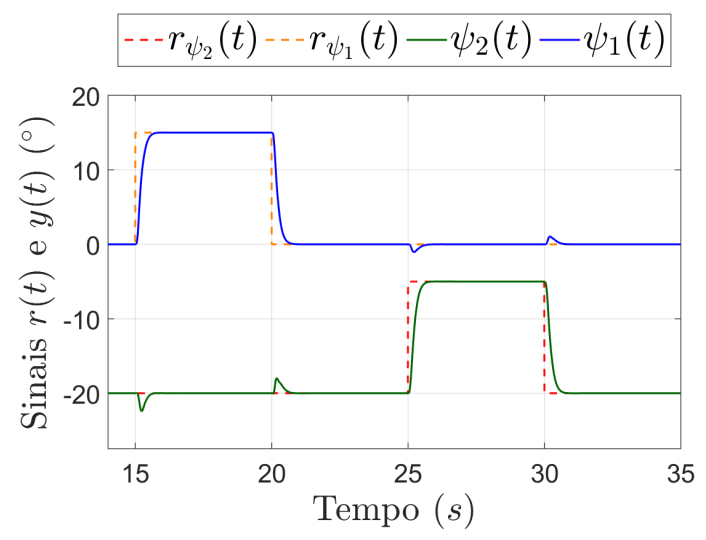

(c) Sinais de Controle $u(t)$ em N.m
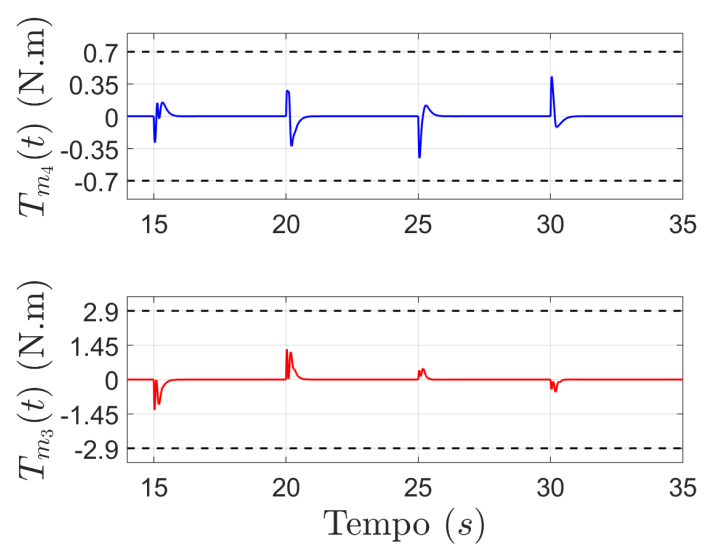

(e) Velocidades Angulares $\omega\left(k T_{s}\right)$ em $\% / \mathrm{s}$
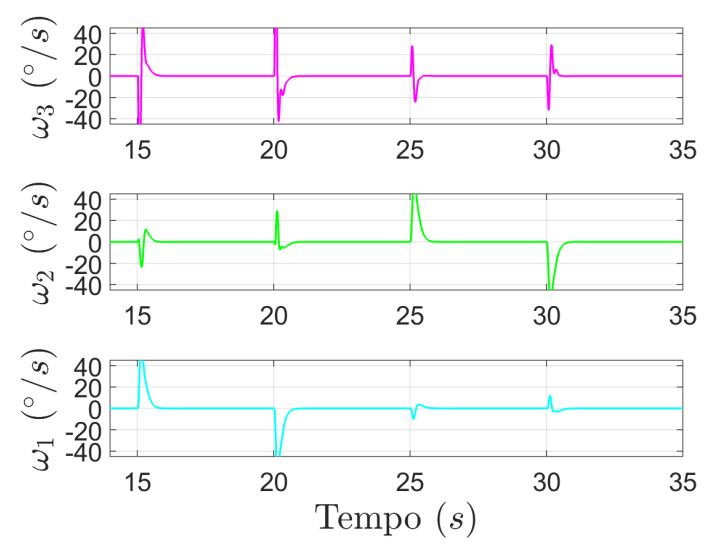

(b) Sinais $r(t)$ e $y(t) \mathrm{em}^{\circ}$

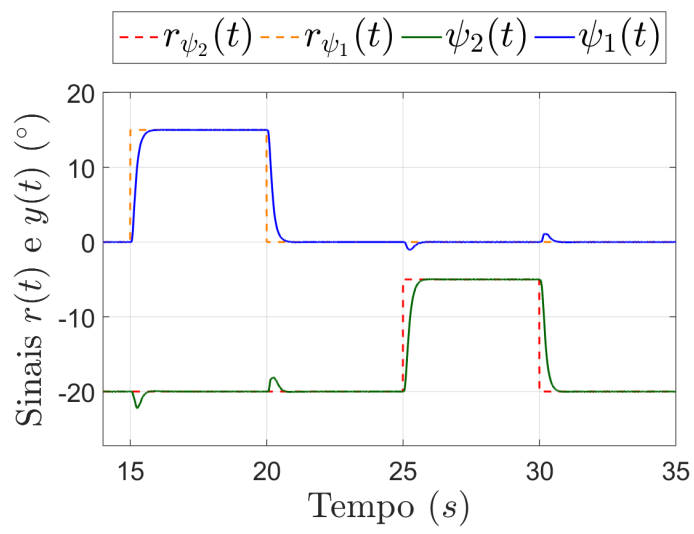

(d) Sinais de Controle $u(t)$ em N.m
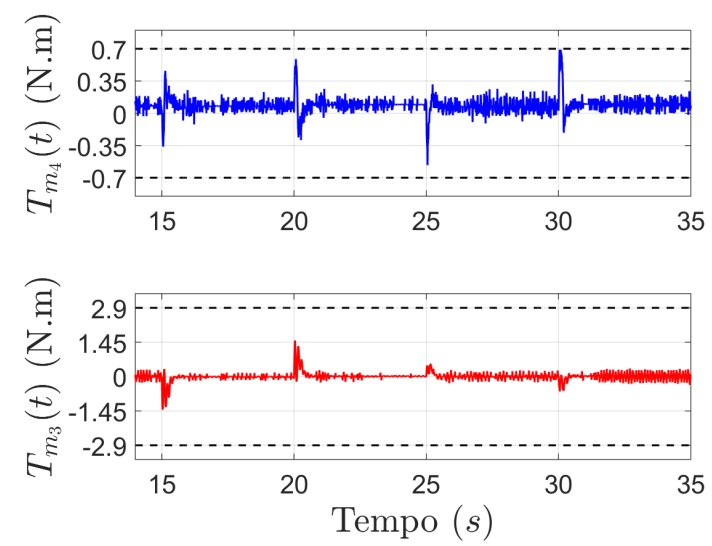

(f) Velocidades Angulares $\omega\left(k T_{s}\right)$ em $\% / \mathrm{s}$
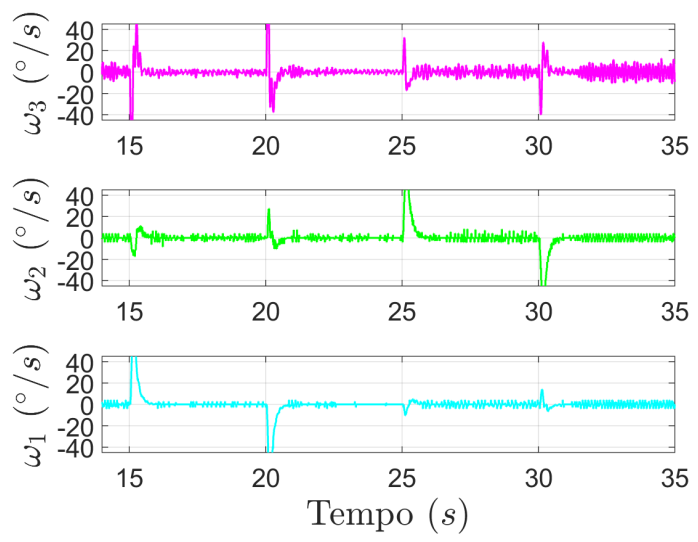

Fonte: Autor. 
Figura 53: Gráficos com os resultados do teste de acompanhamento de referência com sinais do ensaio 1 (negativo) - Sistema acoplado de fase mínima com controle LQR, com os sinais obtidos na simulação [(a), (c) e (e)] e no experimento [(b), (d) e (f)]

(a) Sinais $r(t)$ e $y(t)$ em $^{\circ}$

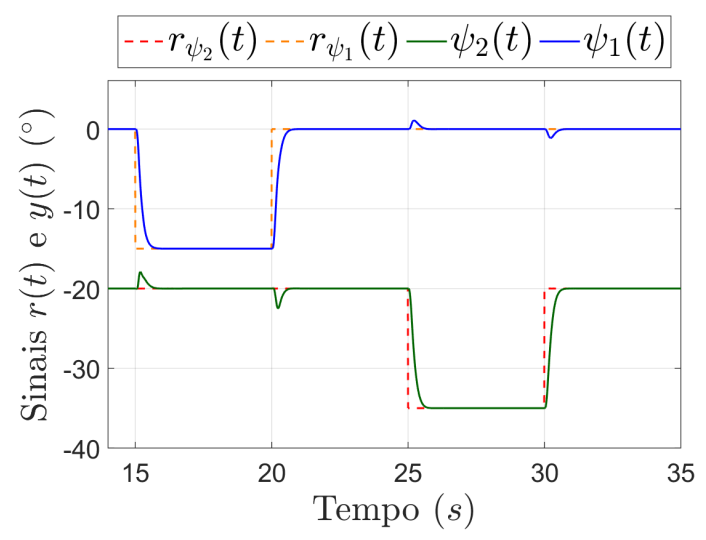

(c) Sinais de Controle $u(t)$ em N.m
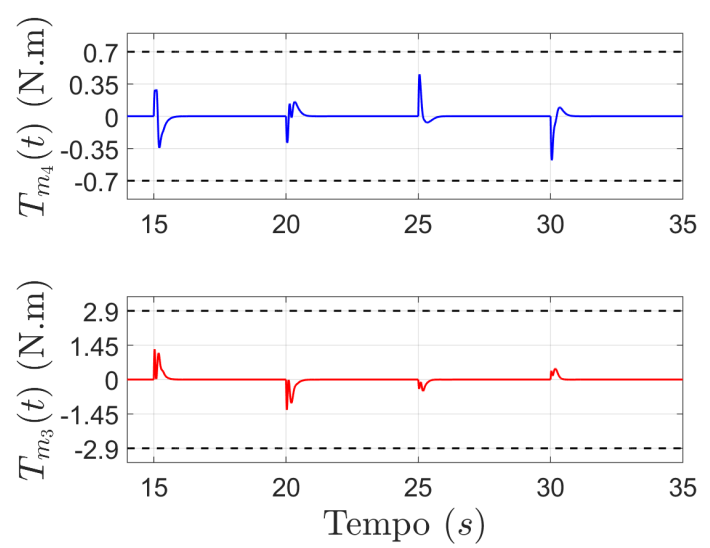

(e) Velocidades Angulares $\omega\left(k T_{s}\right)$ em $\% / \mathrm{s}$
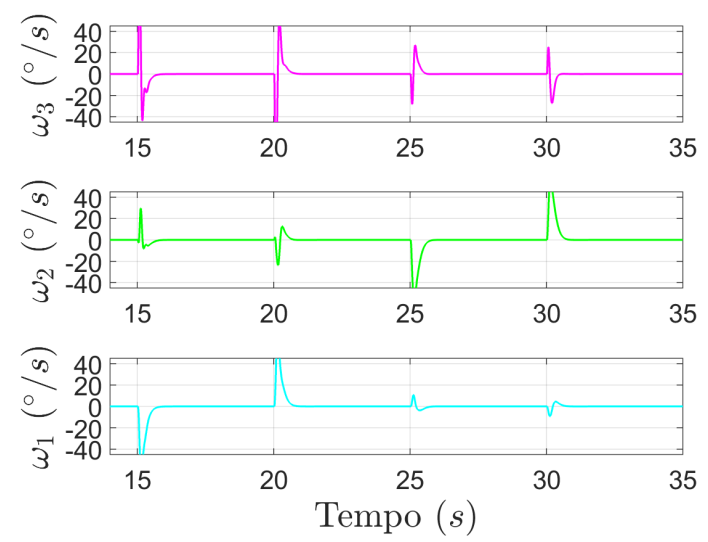

(b) Sinais $r(t)$ e $y(t) \mathrm{em}^{\circ}$

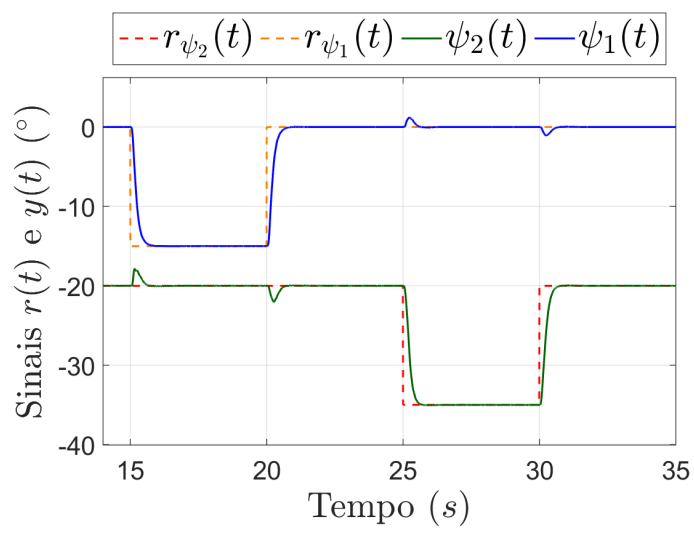

(d) Sinais de Controle $u(t)$ em N.m
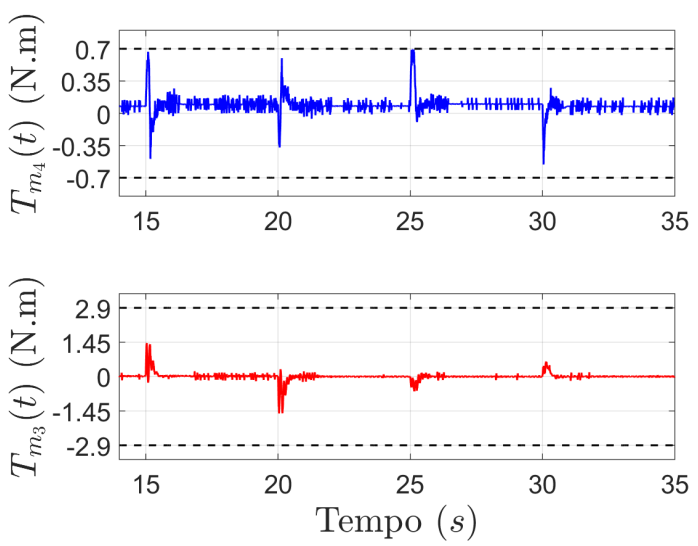

(f) Velocidades Angulares $\omega\left(k T_{s}\right)$ em $\% / \mathrm{s}$
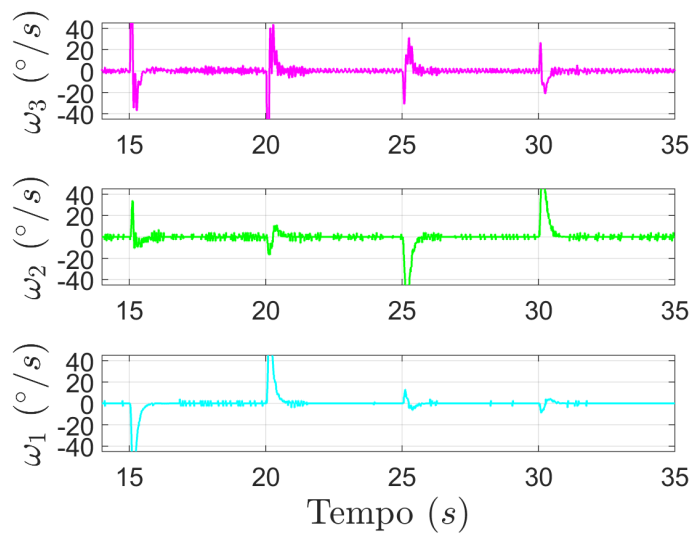

Fonte: Autor. 
Figura 54: Gráficos com os resultados do teste de rejeição à perturbação com sinais do ensaio 1 (positivo) - Sistema acoplado de fase mínima com controle LQR, com os sinais obtidos na simulação [(a), (c) e (e)] e no experimento [(b), (d) e (f)]

(a) Sinais $r(t)$ e $y(t)$ em $^{\circ}$

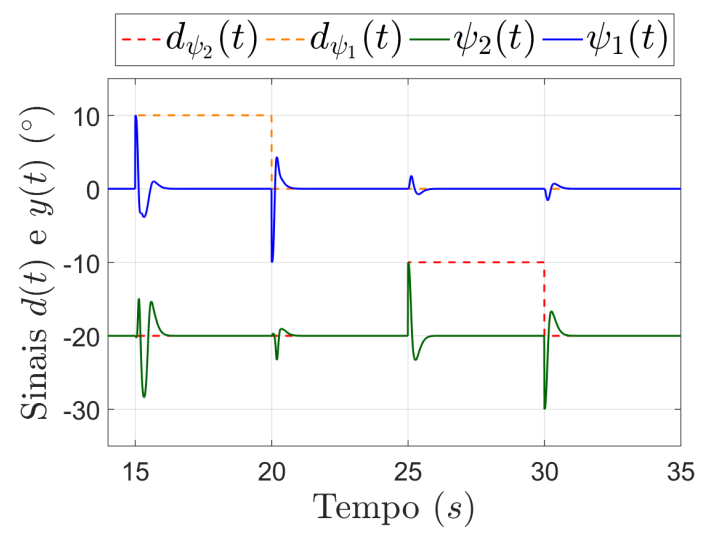

(c) Sinais de Controle $u(t)$ em N.m
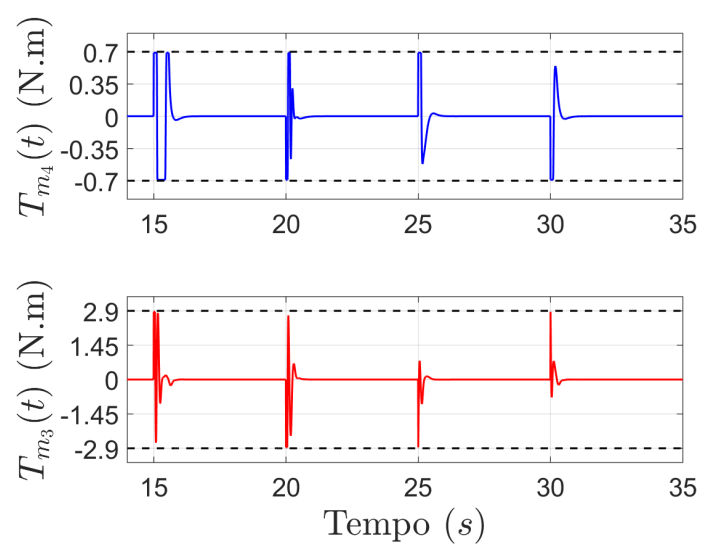

(e) Velocidades Angulares $\omega\left(k T_{s}\right)$ em $\% / \mathrm{s}$
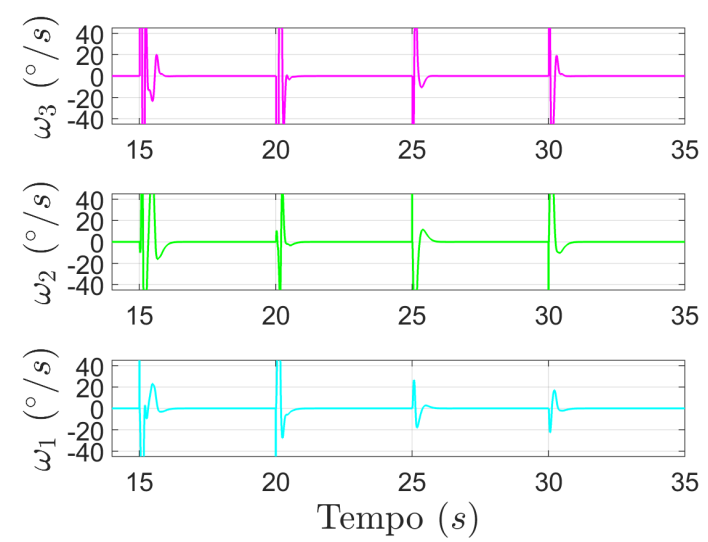

(b) Sinais $r(t)$ e $y(t)$ em $^{\circ}$

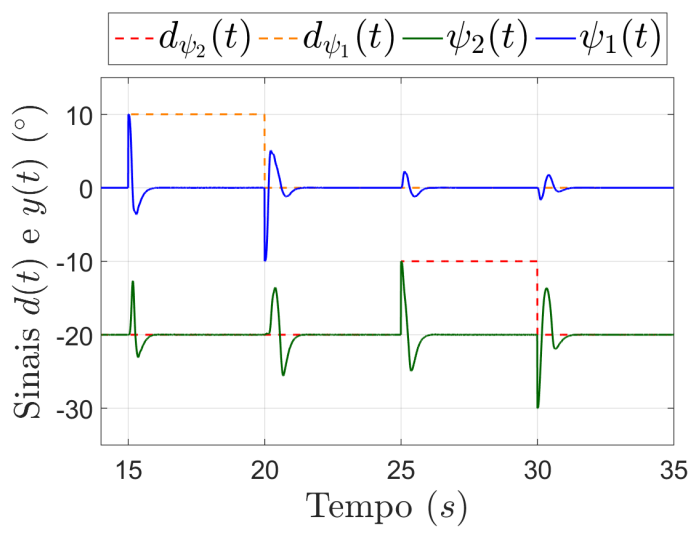

(d) Sinais de Controle $u(t)$ em N.m
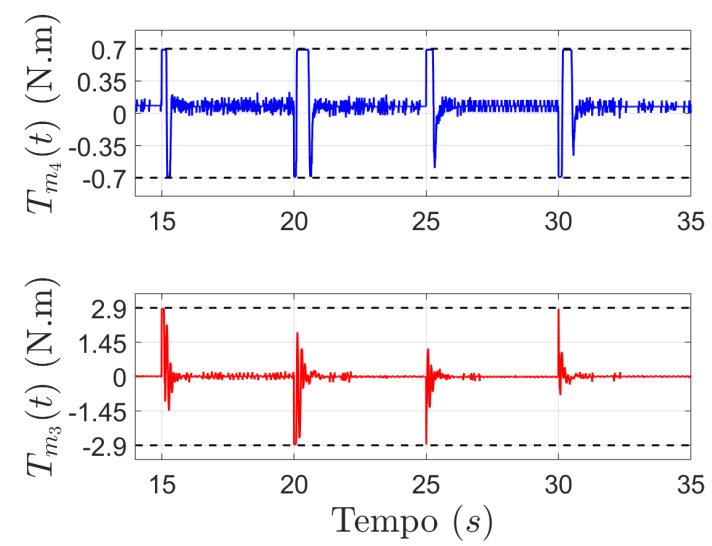

(f) Velocidades Angulares $\omega\left(k T_{s}\right)$ em $\% / \mathrm{s}$
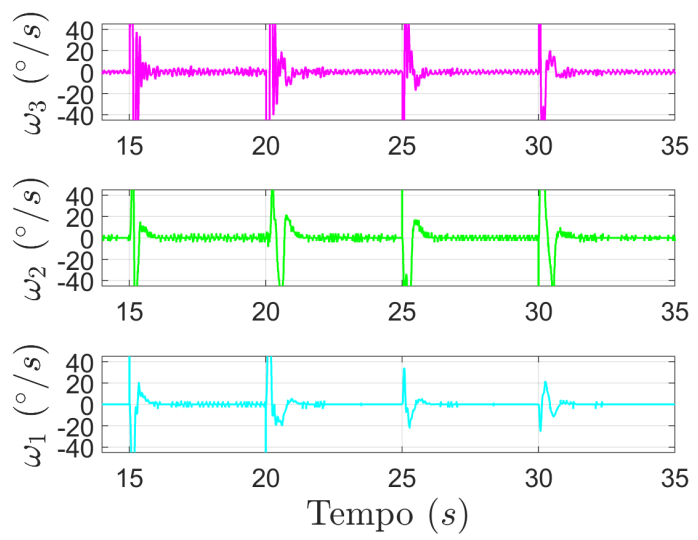

Fonte: Autor. 
Figura 55: Gráficos com os resultados do teste de rejeição à perturbação com sinais do ensaio 1 (negativo) - Sistema acoplado de fase mínima com controle LQR, com os sinais obtidos na simulação [(a), (c) e (e)] e no experimento [(b), (d) e (f)]

(a) Sinais $r(t)$ e $y(t)$ em $^{\circ}$

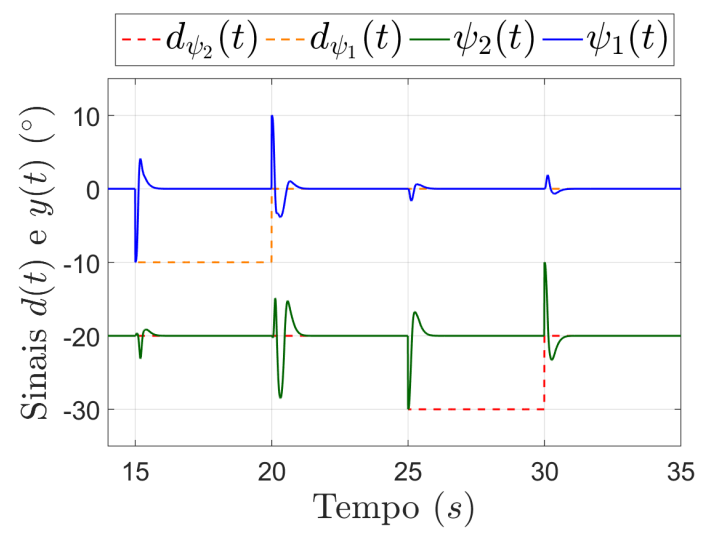

(c) Sinais de Controle $u(t)$ em N.m
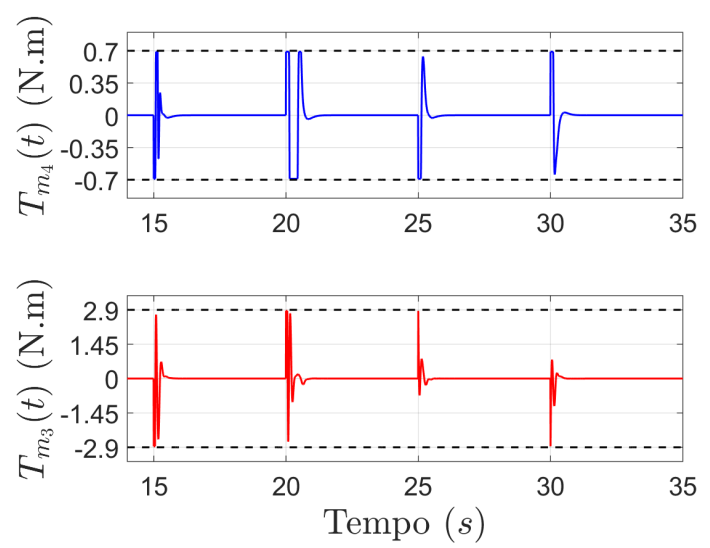

(e) Velocidades Angulares $\omega\left(k T_{s}\right)$ em $\% / \mathrm{s}$
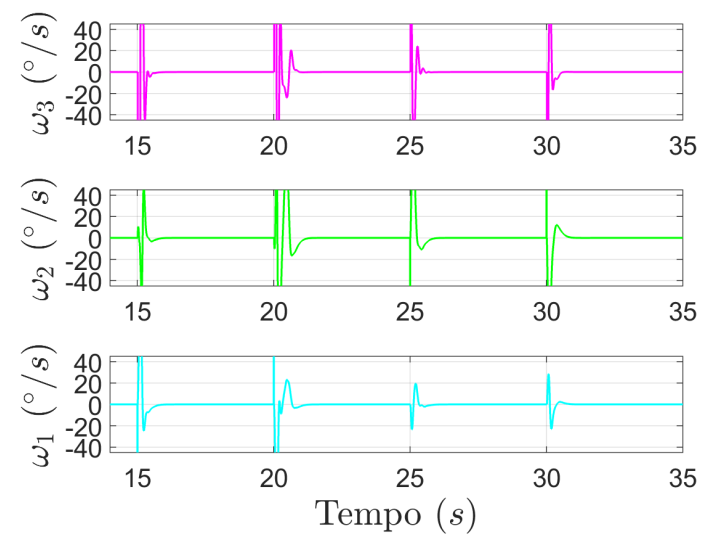

(b) Sinais $r(t)$ e $y(t)$ em $^{\circ}$

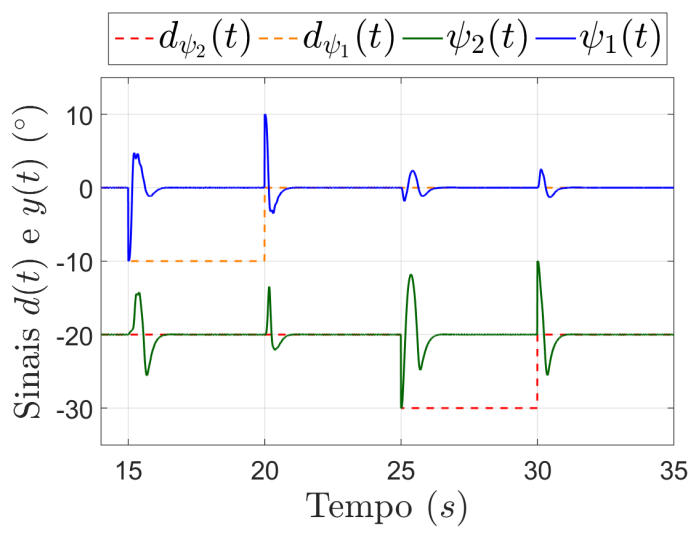

(d) Sinais de Controle $u(t)$ em N.m
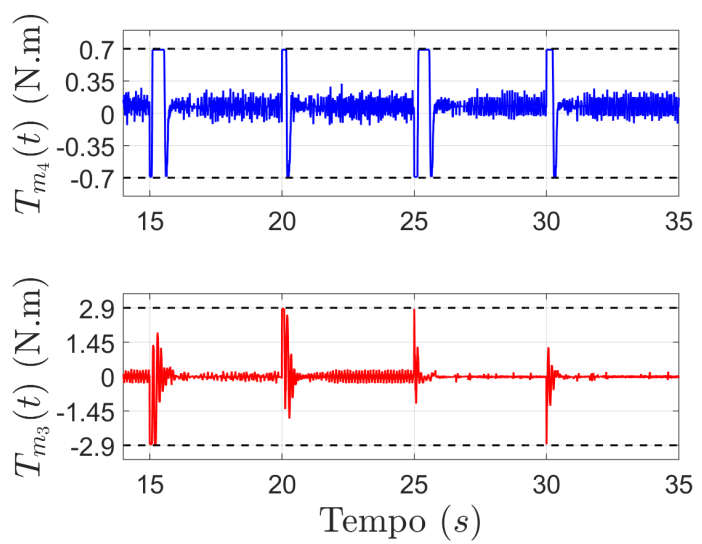

(f) Velocidades Angulares $\omega\left(k T_{s}\right)$ em $\% / \mathrm{s}$
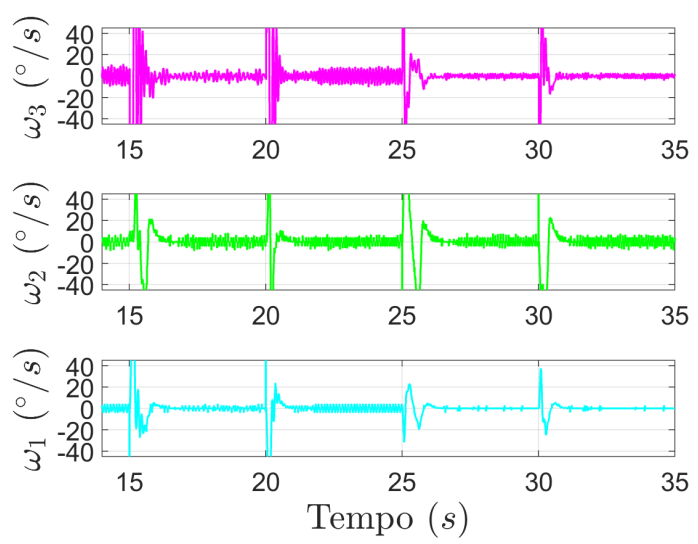

Fonte: Autor. 


\section{H.2.2.2 Ensaio 2 - Pulsos Retangulares Simultâneos}

As Figuras 56 e 57 apresentam os resultados obtidos nos testes de acompanhamento de referência e de rejeição à perturbação, respectivamente.

Figura 56: Gráficos com os resultados do teste de acompanhamento de referência com sinais do ensaio 2 - Sistema acoplado de fase mínima com controle LQR, com os sinais obtidos na simulação [(a), (c) e (e)] e no experimento [(b), (d) e (f)]

(a) Sinais $r(t)$ e $y(t) \mathrm{em}^{\circ}$

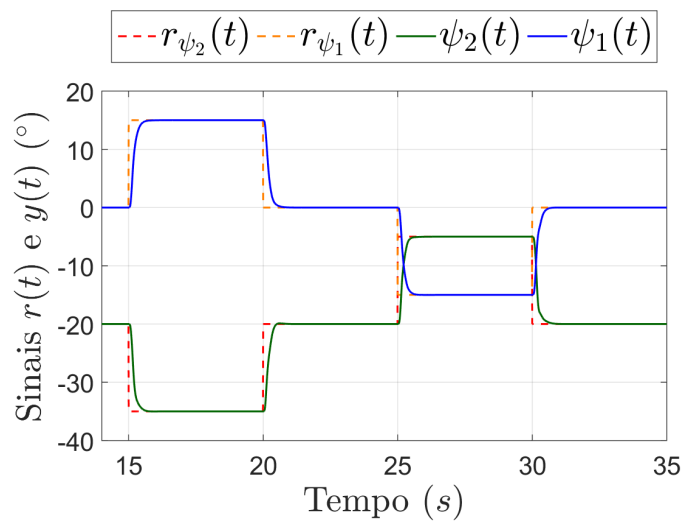

(c) Sinais de Controle $u(t)$ em N.m
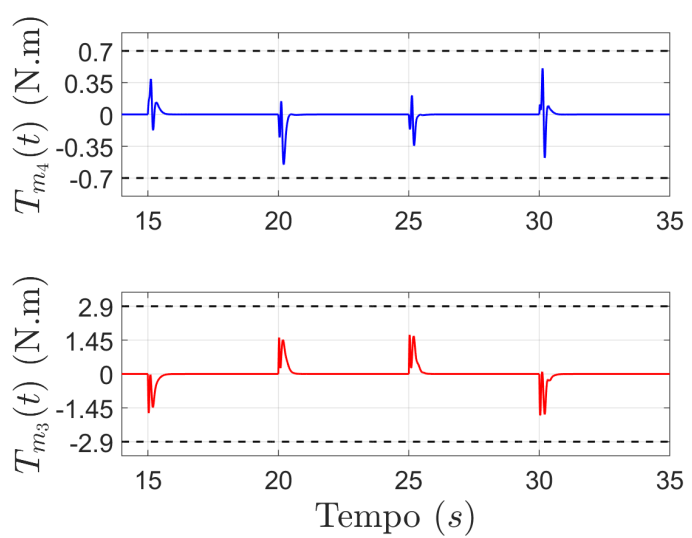

(e) Velocidades Angulares $\omega\left(k T_{s}\right) \mathrm{em} \% \mathrm{~s}$
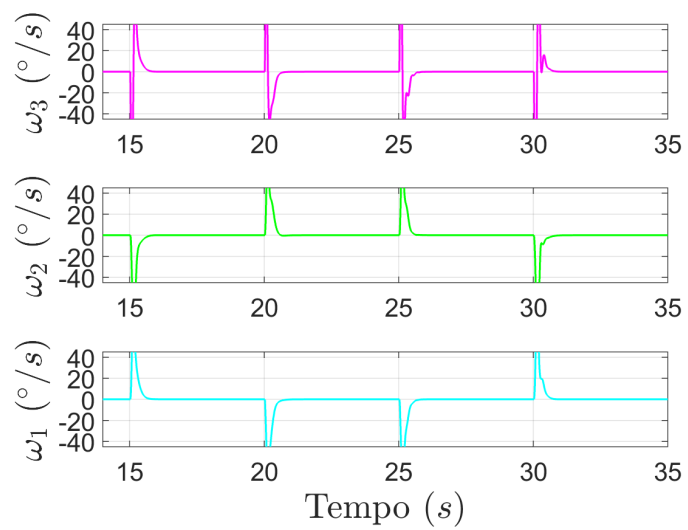

(b) Sinais $r(t)$ e $y(t) \mathrm{em}^{\circ}$

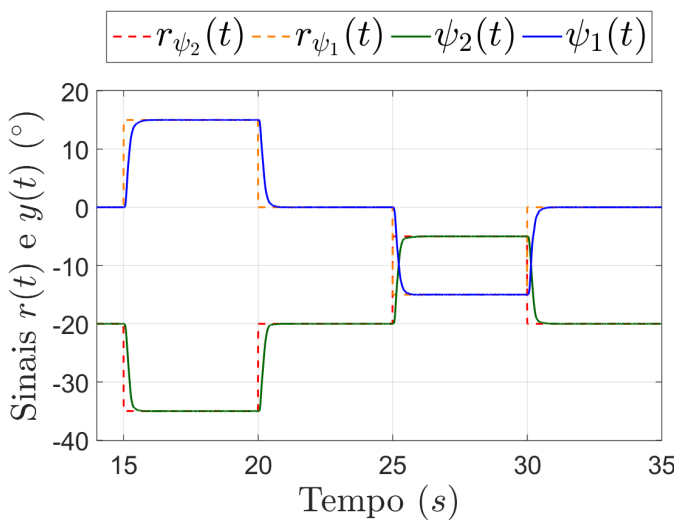

(d) Sinais de Controle $u(t)$ em N.m
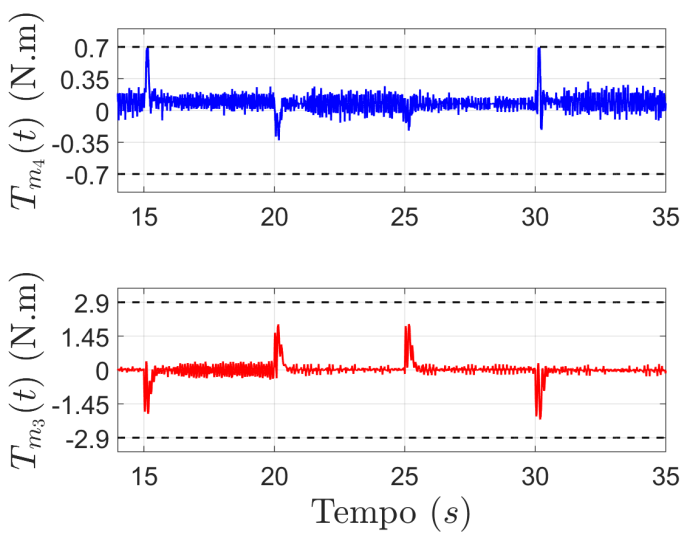

(f) Velocidades Angulares $\omega\left(k T_{s}\right)$ em $\% \mathrm{~s}$
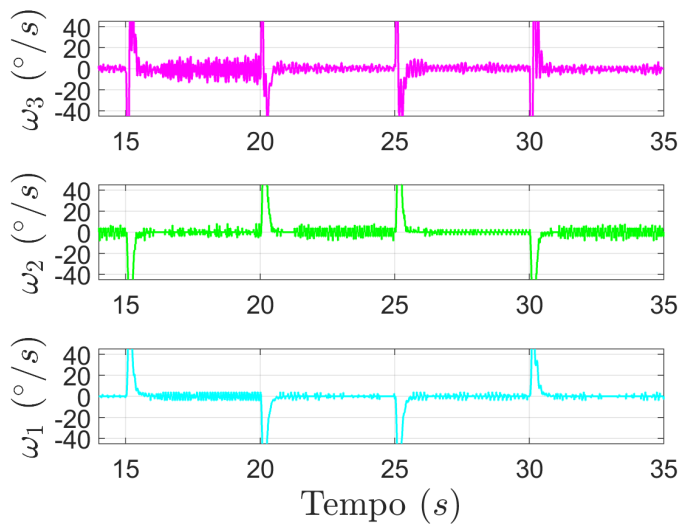

Fonte: Autor. 
Figura 57: Gráficos com os resultados do teste de rejeição à perturbação com sinais do ensaio 2 - Sistema acoplado de fase mínima com controle LQR, com os sinais obtidos na simulação [(a), (c) e (e)] e no experimento [(b), (d) e (f)]
(a) Sinais $r(t)$ e $y(t) \mathrm{em}^{\circ}$
(b) Sinais $r(t)$ e $y(t) \mathrm{em}^{\circ}$

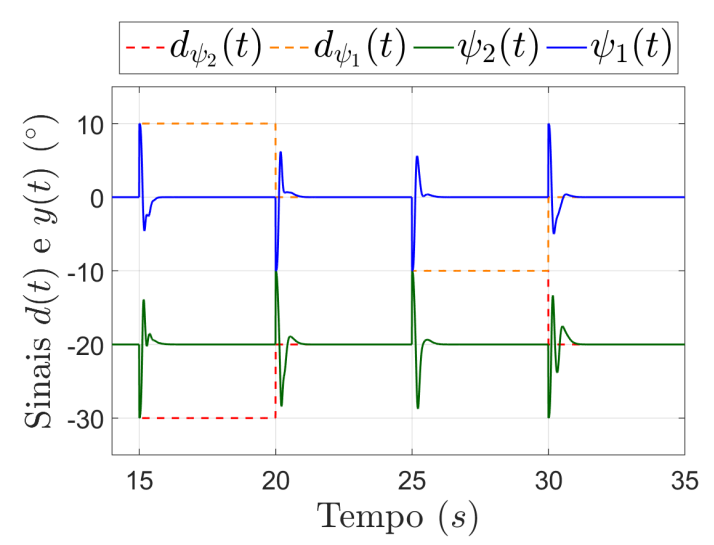

(c) Sinais de Controle $u(t)$ em N.m
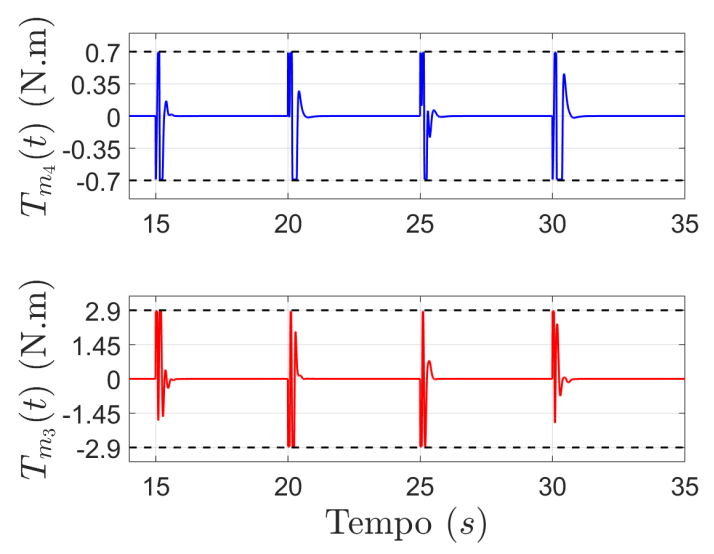

(e) Velocidades Angulares $\omega\left(k T_{s}\right)$ em $\% / \mathrm{s}$
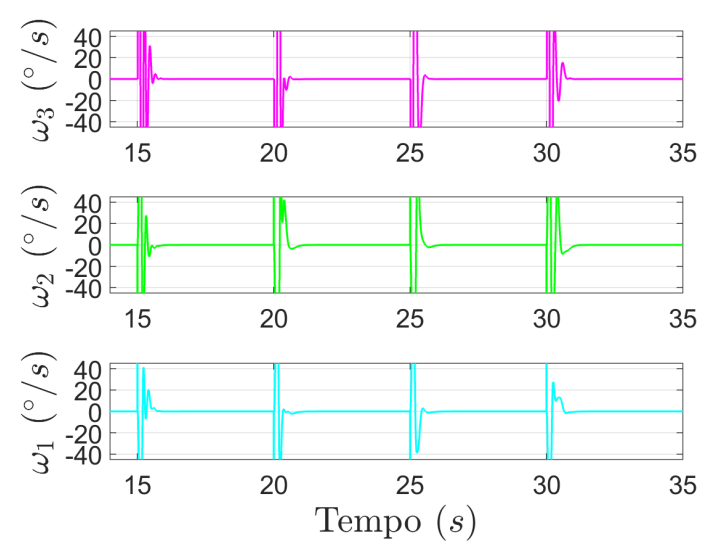

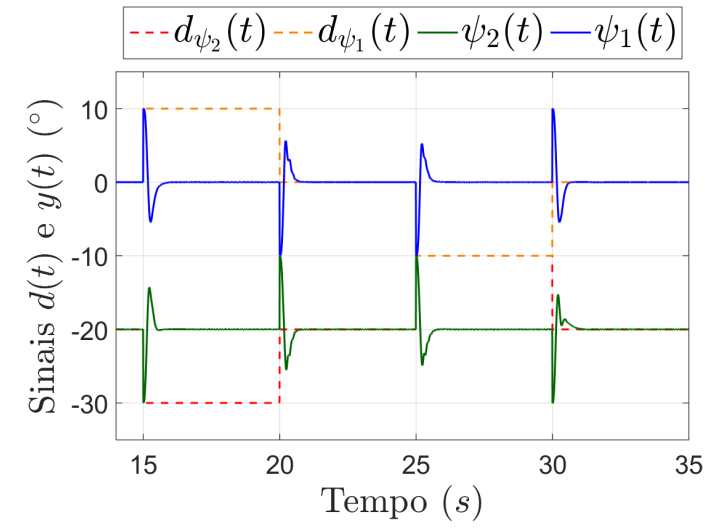

(d) Sinais de Controle $u(t)$ em N.m
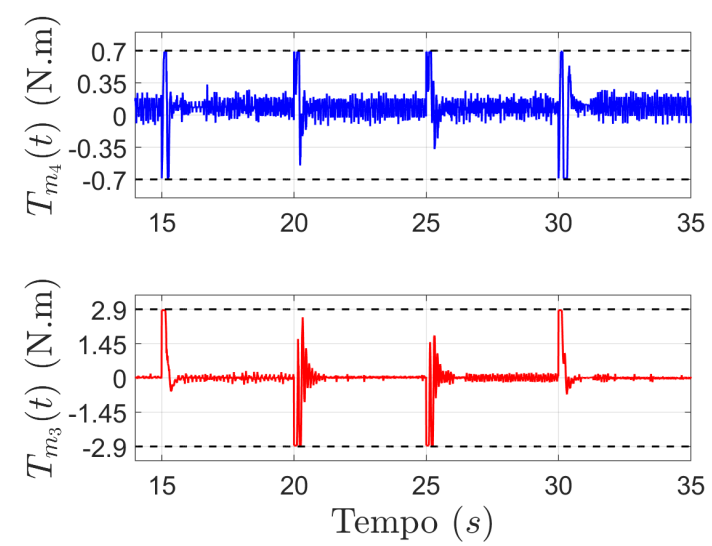

(f) Velocidades Angulares $\omega\left(k T_{s}\right) \mathrm{em} \% \mathrm{~s}$
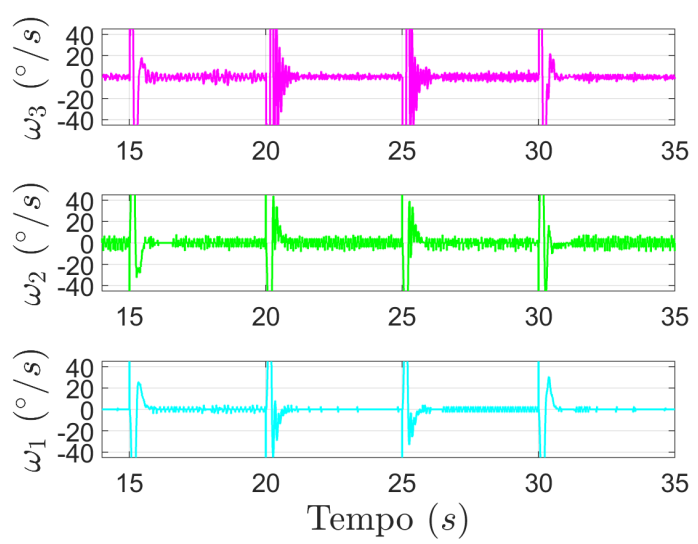

Fonte: Autor. 


\section{H.2.2.3 Ensaio 3 - Sinais Senoidais}

As Figuras 58 e 59 apresentam os resultados obtidos nos testes de acompanhamento de referência e de rejeição à perturbação, respectivamente.

Figura 58: Gráficos com os resultados do teste de acompanhamento de referência com sinais do ensaio 3 - Sistema acoplado de fase mínima com controle LQR, com os sinais obtidos na simulação [(a), (c) e (e)] e no experimento [(b), (d) e (f)]

(a) Sinais $r(t)$ e $y(t) \mathrm{em}^{\circ}$

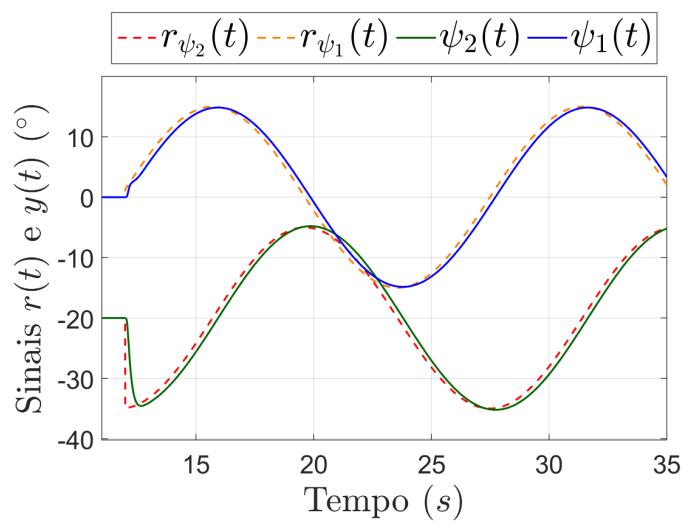

(c) Sinais de Controle $u(t)$ em N.m
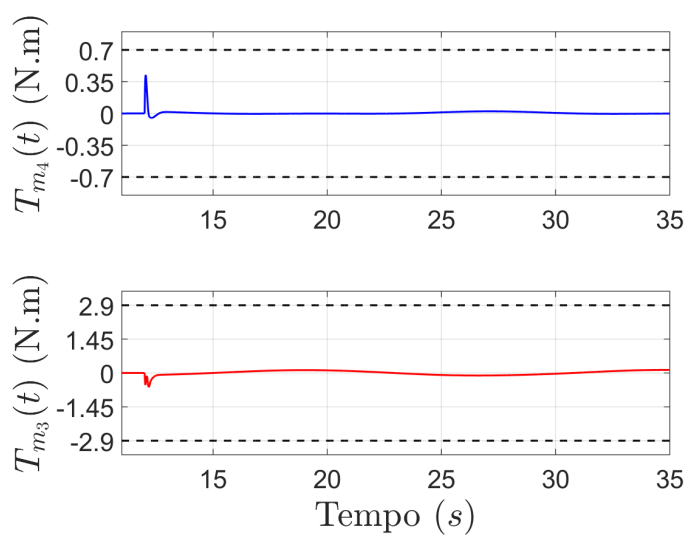

(e) Velocidades Angulares $\omega\left(k T_{s}\right) \mathrm{em} \% \mathrm{~s}$
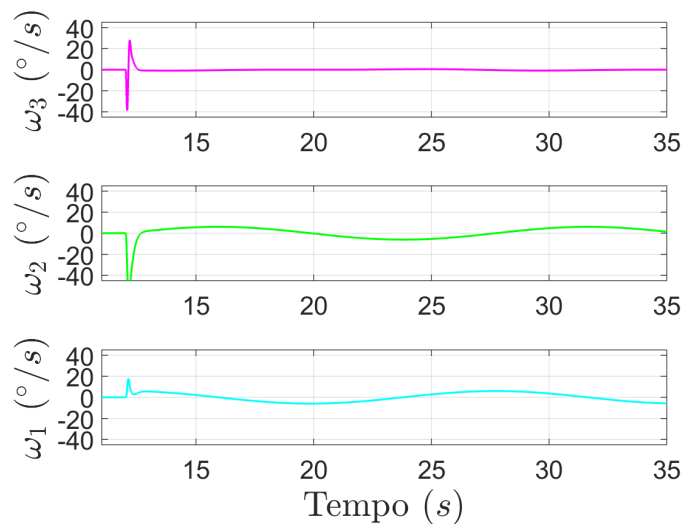

(b) Sinais $r(t)$ e $y(t) \mathrm{em}^{\circ}$

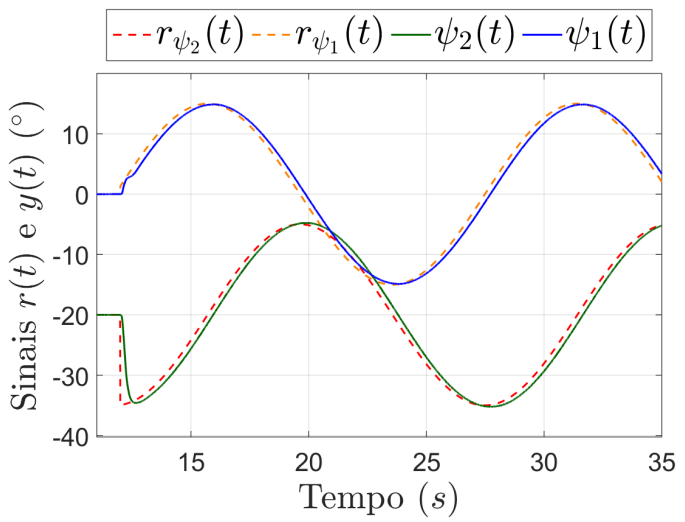

(d) Sinais de Controle $u(t)$ em N.m
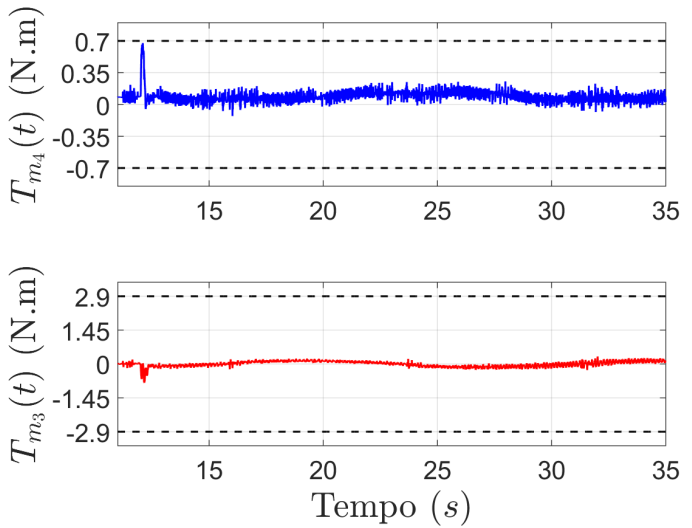

(f) Velocidades Angulares $\omega\left(k T_{s}\right) \mathrm{em}^{\circ} / \mathrm{s}$
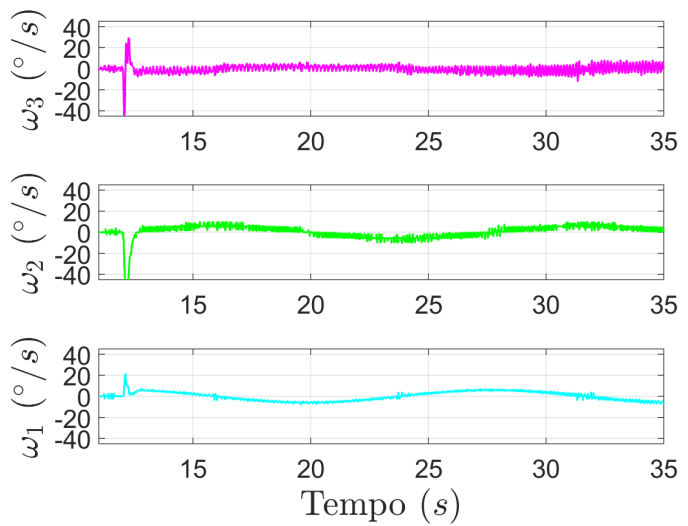

Fonte: Autor. 
Figura 59: Gráficos com os resultados do teste de rejeição à perturbação com sinais do ensaio 3 - Sistema acoplado de fase mínima com controle LQR, com os sinais obtidos na simulação [(a), (c) e (e)] e no experimento [(b), (d) e (f)]
(a) Sinais $r(t)$ e $y(t) \mathrm{em}^{\circ}$
(b) Sinais $r(t)$ e $y(t) \mathrm{em}^{\circ}$

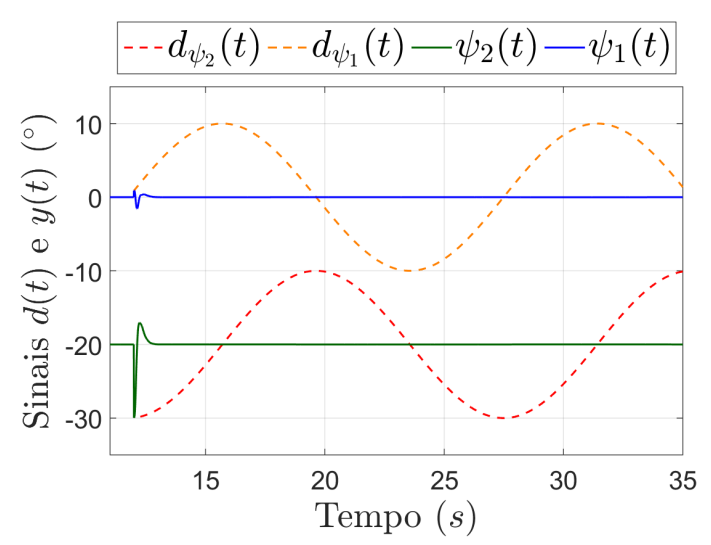

(c) Sinais de Controle $u(t)$ em N.m
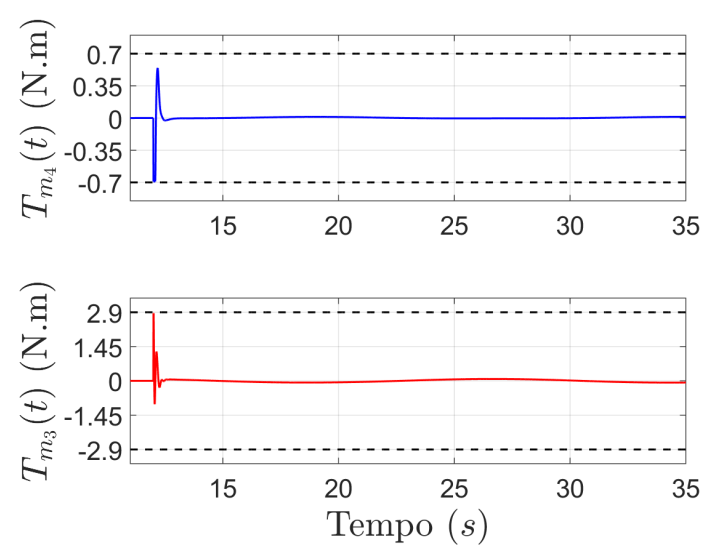

(e) Velocidades Angulares $\omega\left(k T_{s}\right)$ em $\% / \mathrm{s}$
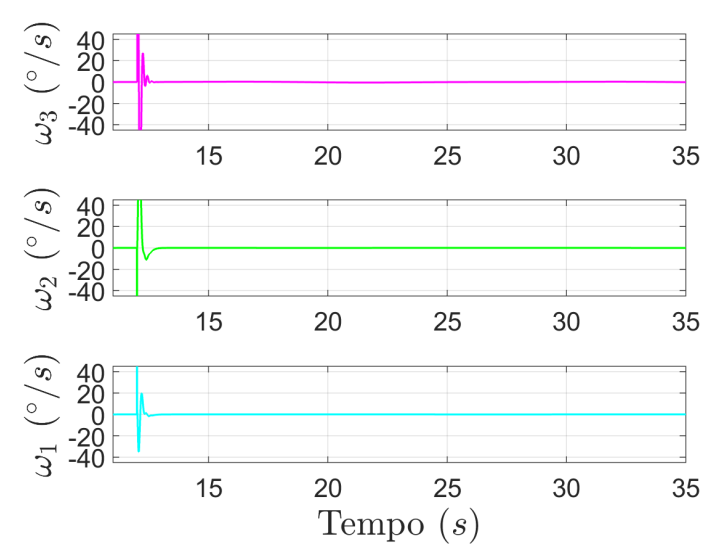

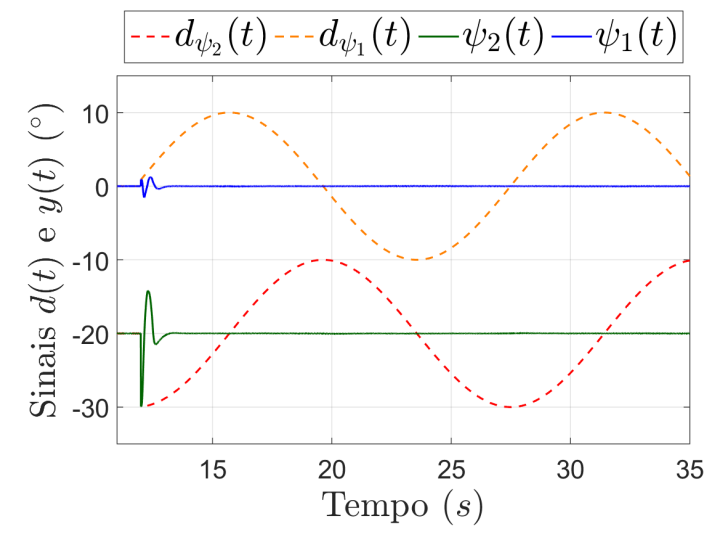

(d) Sinais de Controle $u(t)$ em N.m
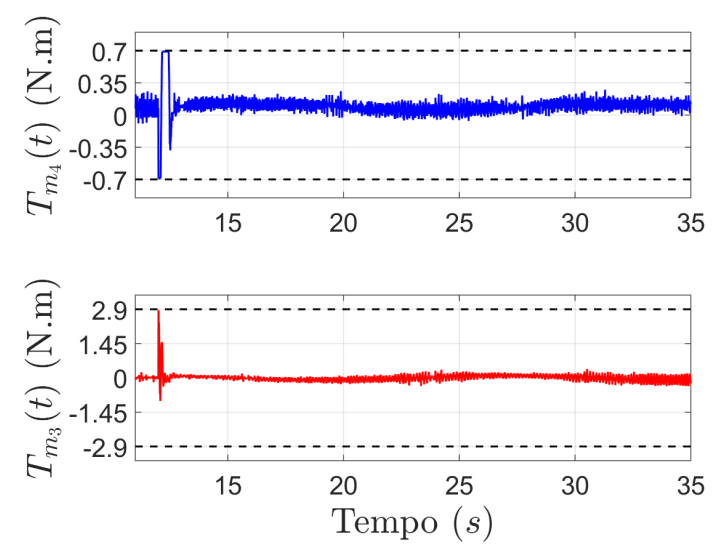

(f) Velocidades Angulares $\omega\left(k T_{s}\right) \mathrm{em} \% \mathrm{~s}$
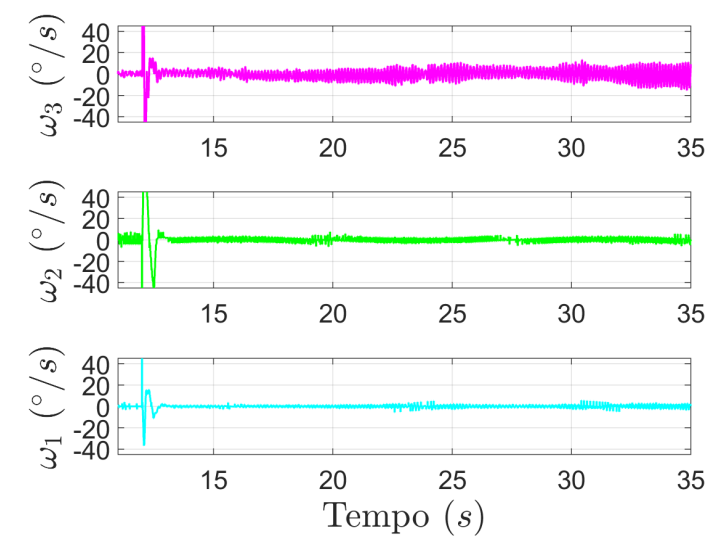

Fonte: Autor. 


\section{H.3 Caso Acoplado de Fase Não-Mínima}

Para o sistema acoplado de fase não-mínima realiza-se o procedimento experimental, somente para o teste de acompanhamento de referência com sinais do ensaio 2 (pulsos retangulares simultâneos), com os sistemas contendo a planta na configuração acoplada de fase não-mínima (no ponto de operação $\psi_{2_{0}}=20^{\circ}, \psi_{3_{0}}=-20^{\circ}$ e $\Omega=400 \mathrm{RPM}$ ) e os controladores digitais projetados.

\section{H.3.1 Sistema com o Controlador PID}

Para o sistema acoplado de fase não-mínima, o controlador digital PID utilizado é projetado em relação ao modelo acoplado de fase não-mínima da planta. Isso se deve ao fato do controlador projetado em relação ao modelo desacoplado não ser adequado a este caso, pois a matriz de transferência $G(z)$ do modelo acoplado possui um dos elementos da diagonal principal com sinal positivo, enquanto que os dois elementos da diagonal da matriz de transferência do modelo desacoplado possuem ambos sinais negativos, o que impossibilita o uso do controlador projetado em relação ao modelo desacoplado. Mesmo realizando o projeto do controlador em relação ao modelo acoplado de fase não-mínima, não foi possível controlar o sistema.

\section{H.3.2 Sistema com o Controlador LQR}

Para o sistema acoplado de fase não-mínima, o controlador digital LQR utilizado é projetado em relação ao modelo acoplado de fase não-mínima da planta. Os resultados obtidos nos testes de acompanhamento de referência com os sinais do ensaio 2 são apresentados na Figura 60. 
Figura 60: Gráficos com os resultados do teste de acompanhamento de referência com sinais do ensaio 2 - Sistema acoplado de fase não-mínima com controle LQR, com os sinais obtidos na simulação [(a), (c) e (e)] e no experimento [(b), (d) e (f)]

(a) Sinais $r(t)$ e $y(t)$ em $^{\circ}$

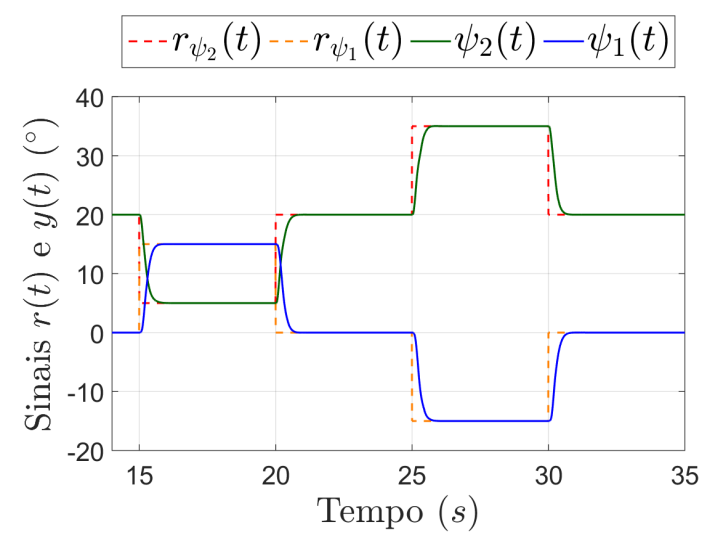

(c) Sinais de Controle $u(t)$ em N.m
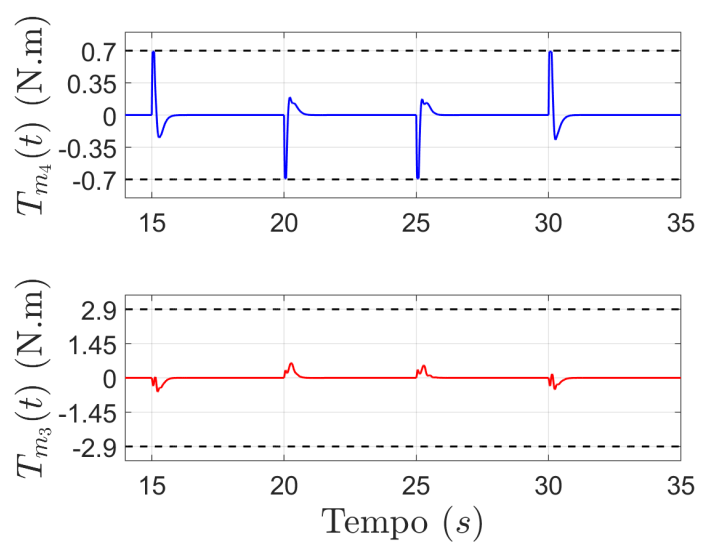

(e) Velocidades Angulares $\omega\left(k T_{s}\right)$ em $\% / \mathrm{s}$
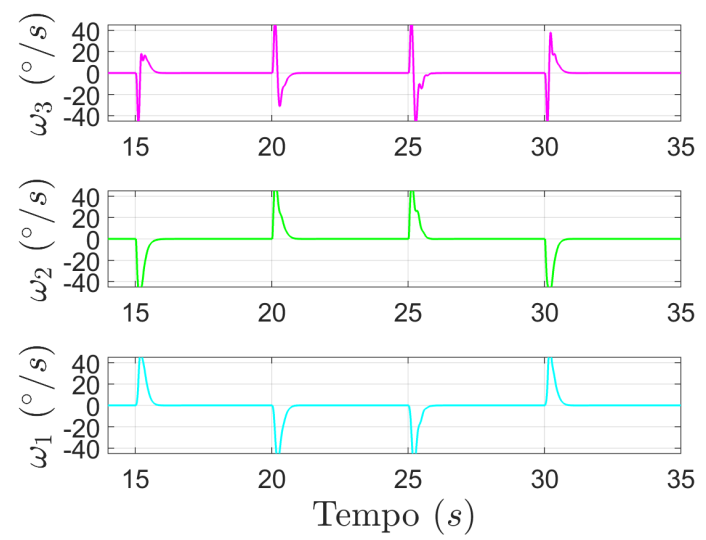

(b) Sinais $r(t)$ e $y(t) \mathrm{em}^{\circ}$

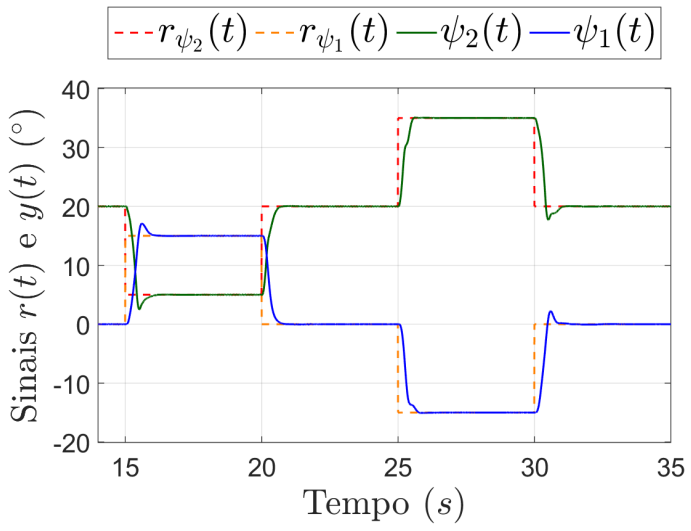

(d) Sinais de Controle $u(t)$ em N.m
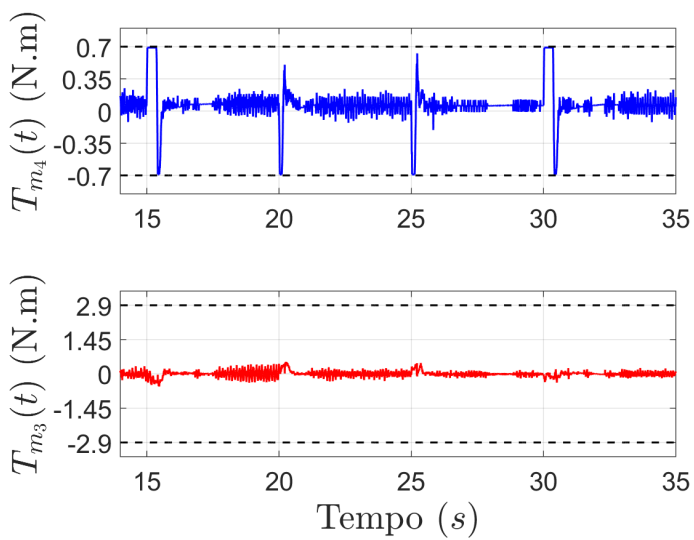

(f) Velocidades Angulares $\omega\left(k T_{s}\right)$ em $\% / \mathrm{s}$
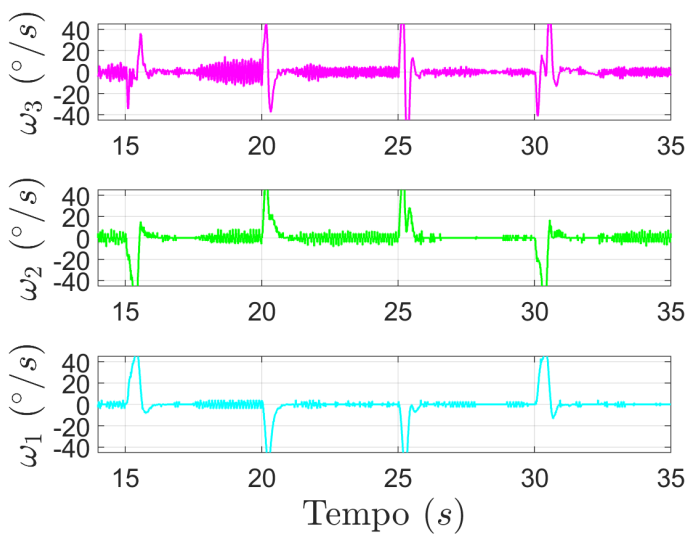

Fonte: Autor. 


\section{APÊNDICE I - DIAGRAMA DE BLOCOS CRIADOS NO SIMULINK}

As Figuras 61 e 62 apresentam, respectivamente, os diagramas de blocos montados no Simulink para a simulação e o experimento prático dos sistemas com o controlador PID projetado, assim como as Figuras 63 e 64 apresentam, respectivamente, os diagramas de blocos montados no Simulink para a simulação e o experimento prático dos sistemas com o controlador LQR projetado. Estes diagramas de blocos possuem alguns subsistemas, que estão ilustrados nas Figuras 65 e 66.

As Figuras 65a e 65b apresentam os subsistemas com o diagrama de blocos da planta, utilizados para a simulação e para o experimento, respectivamente. Dentro destes subsistemas da planta, há mais dois subsistemas, um com o diagrama de blocos do controlador PID, ilustrado na Figura 66a, e outro com o diagrama de blocos da realimentação de velocidade da planta, realizada para o controle PID, ilustrado na Figura 66b.

A Figura 66c apresenta o diagrama de blocos com o controle PI simples utilizado, em todos os sistemas (tanto com o controlador PID quanto com LQR), para elevar e manter a velocidade do disco, a uma dada velocidade w4 dada em RPM, no começo do procedimento experimental.

E o algoritmo do bloco Matlab Function, com as equações de diferenças do controlador LQR, está apresentado na Figura 67.

Todos os dados utilizados nestes diagramas de blocos (como os dados do modelo da planta, do controlador projetado, dos sinais de referência e de perturbação, dentre outros) são gerados por algoritmos criados em arquivos MATLAB ${ }^{\circledR}$, para o controle PID e LQR, apresentados no Apêndice J. 
Figura 61: Diagrama de blocos do Simulink para a simulação do sistema com o controlador PID

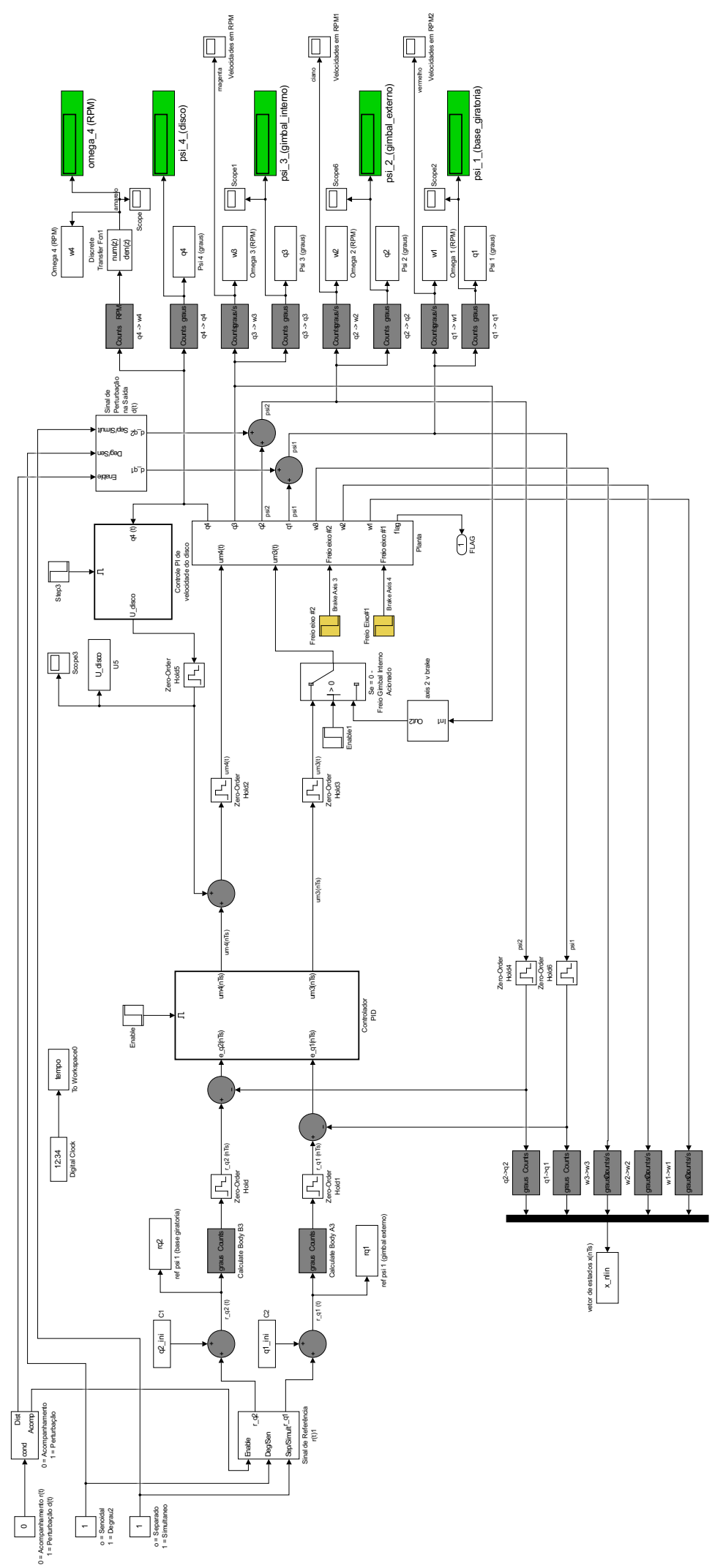

Fonte: Autor. 
Figura 62: Diagrama de blocos do Simulink para o experimento do sistema com o controlador PID

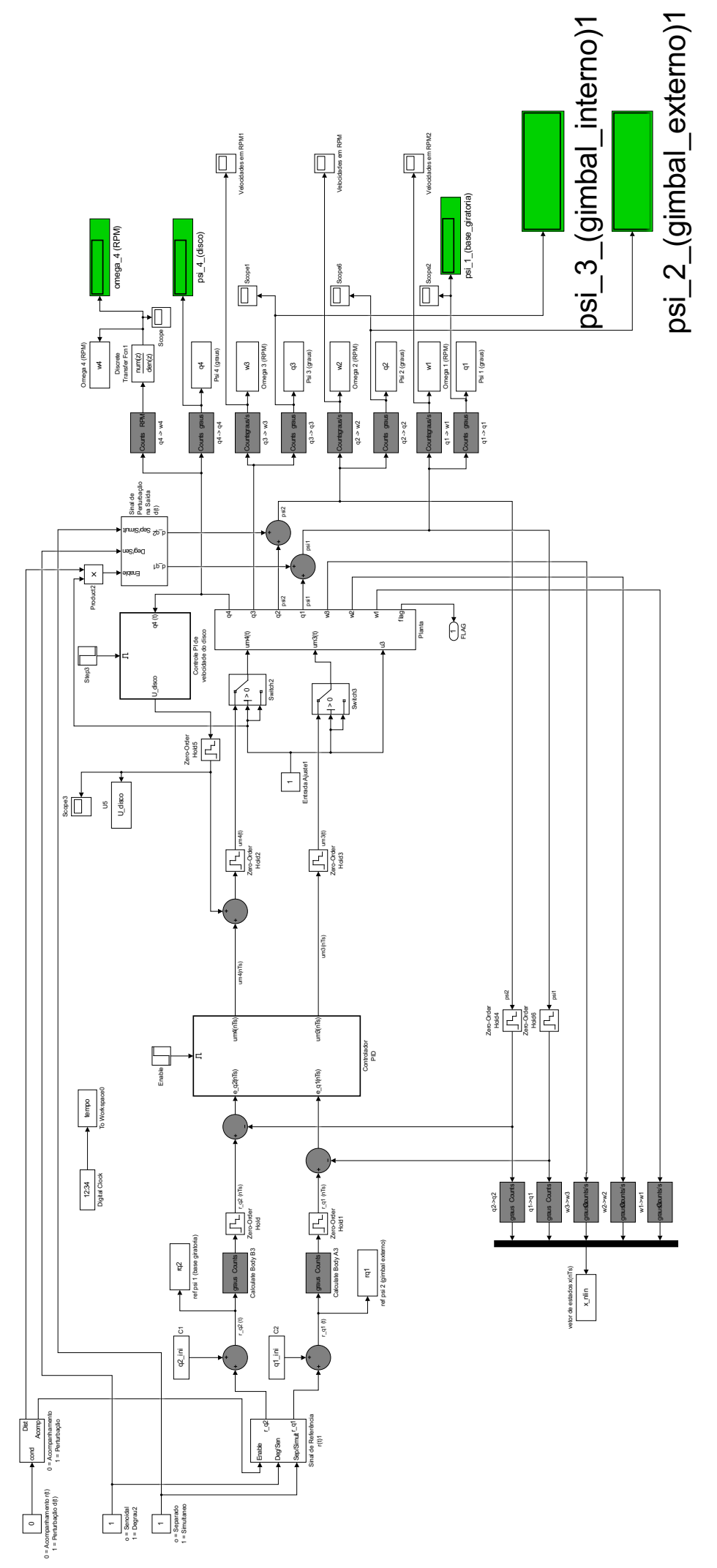

Fonte: Autor. 
Figura 63: Diagrama de blocos do Simulink para a simulação do sistema com o controlador LQR

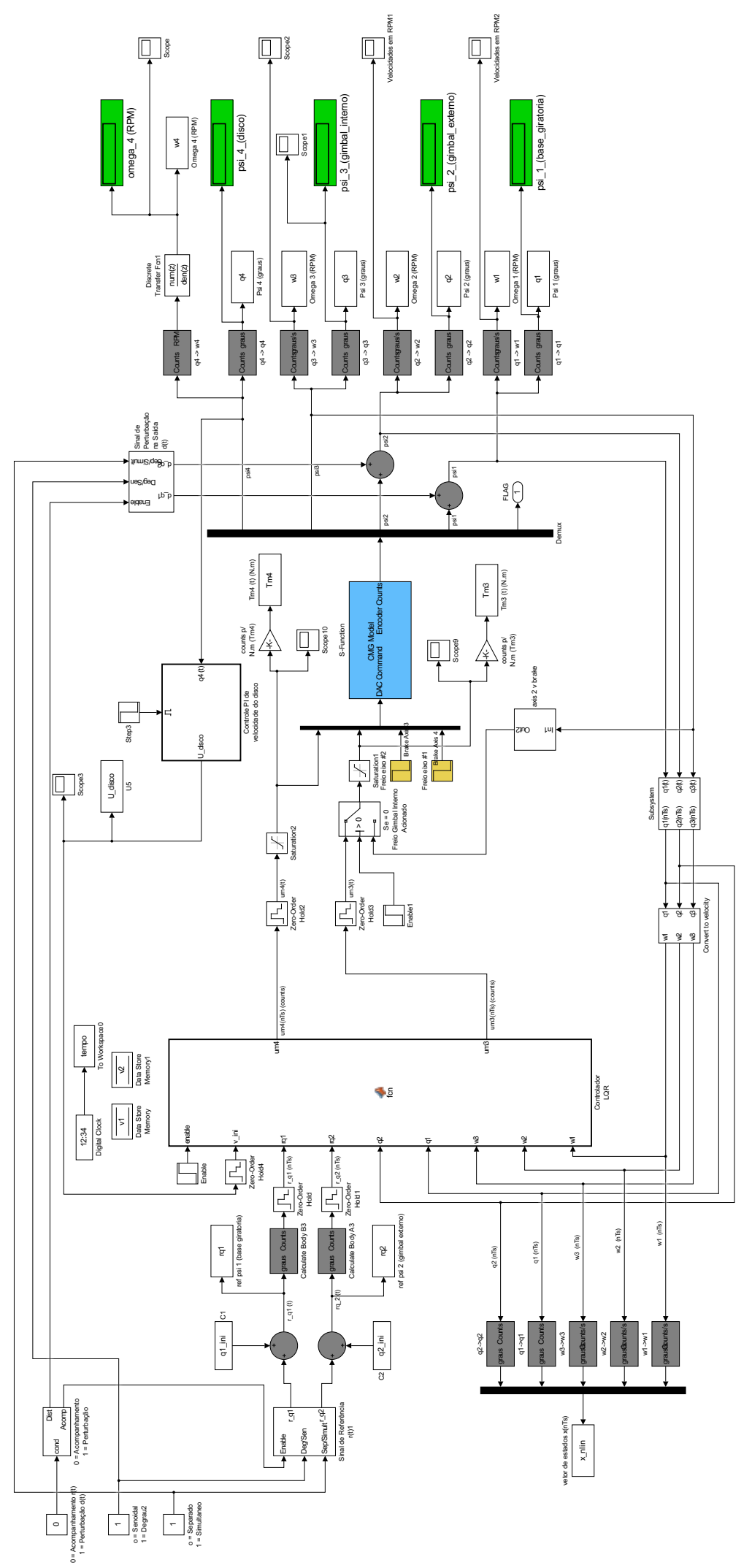

Fonte: Autor. 
Figura 64: Diagrama de blocos do Simulink para o experimento do sistema com o controlador LQR

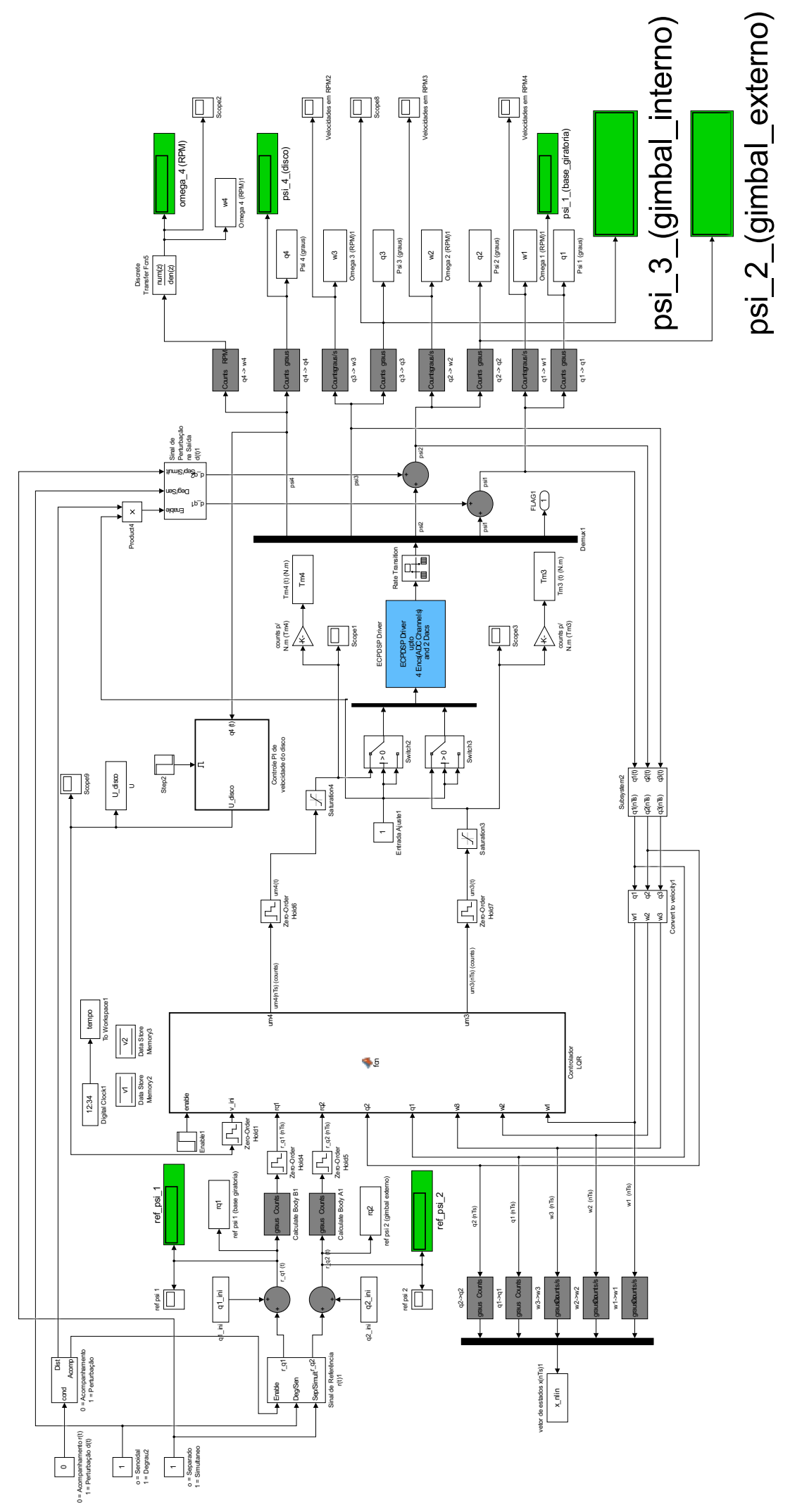

Fonte: Autor. 
Figura 65: Subsistemas com o diagrama de blocos da planta, utilizado para a simulação (a) e para o experimento (b) do sistema com o controlador PID

(a) Subsistema "Planta" da Figura 61

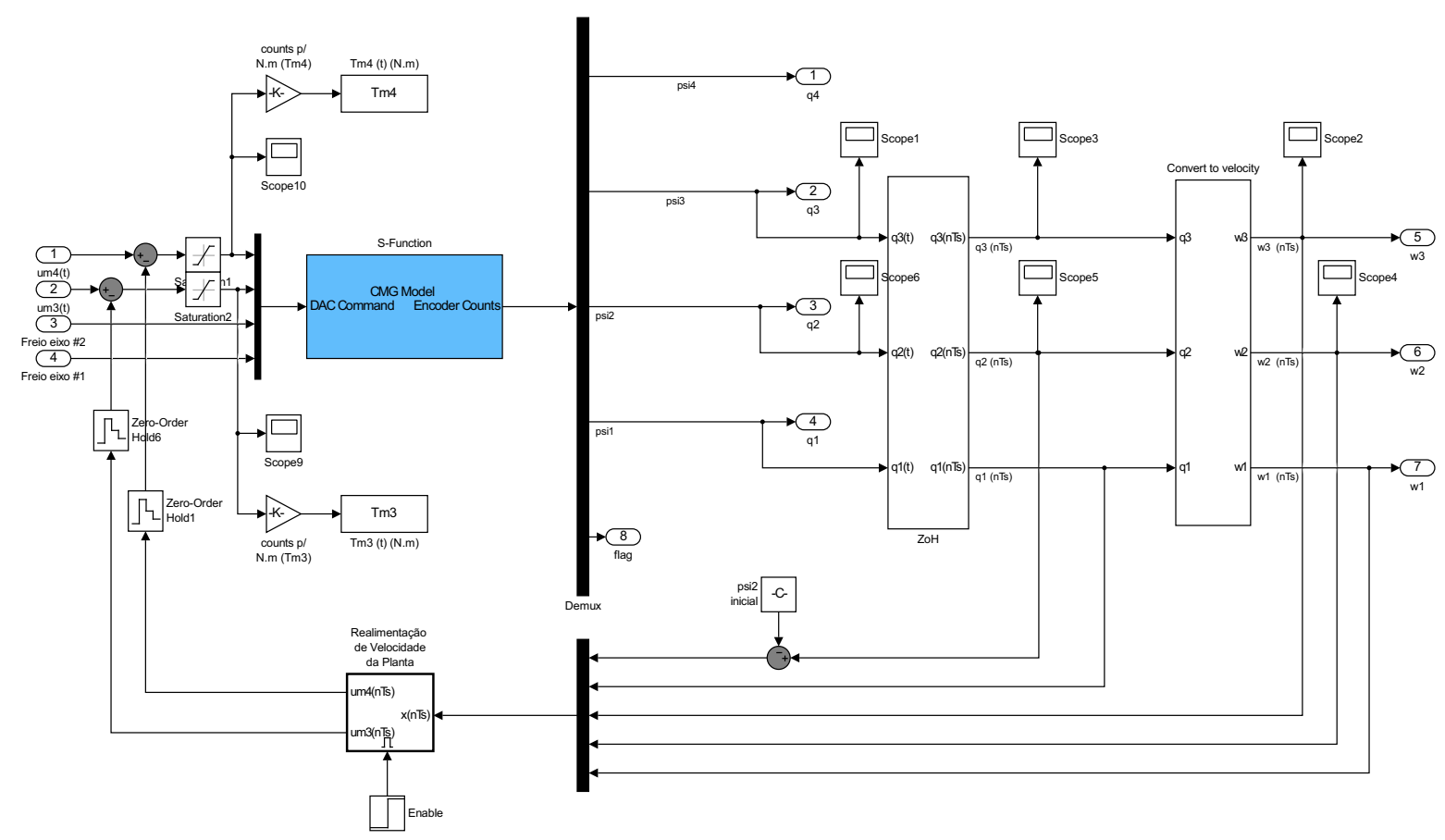

(b) Subsistema "Planta" da Figura 62

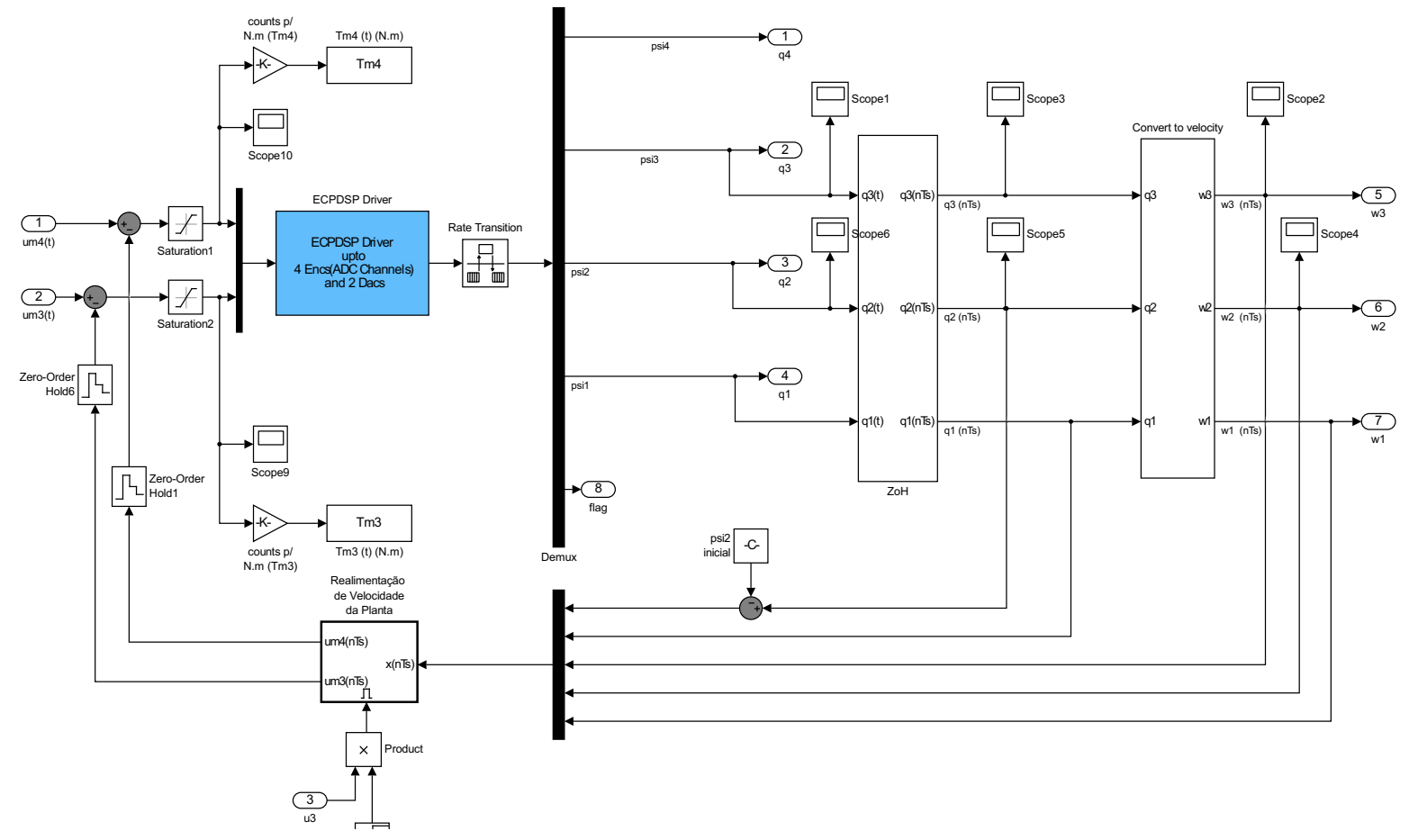

Fonte: Autor. 
Figura 66: Subsistemas com o diagrama de blocos do controlador PID (a), da realimentação de velocidade da planta realizada para o controle PID (b), e do controle PI simples de velocidade do disco.
(a) Subsistema "Controlador PID" das Figu-
(b) Subsistema "Realimentação de Velocidade ras 61 e 62 da Planta" da Figura 65
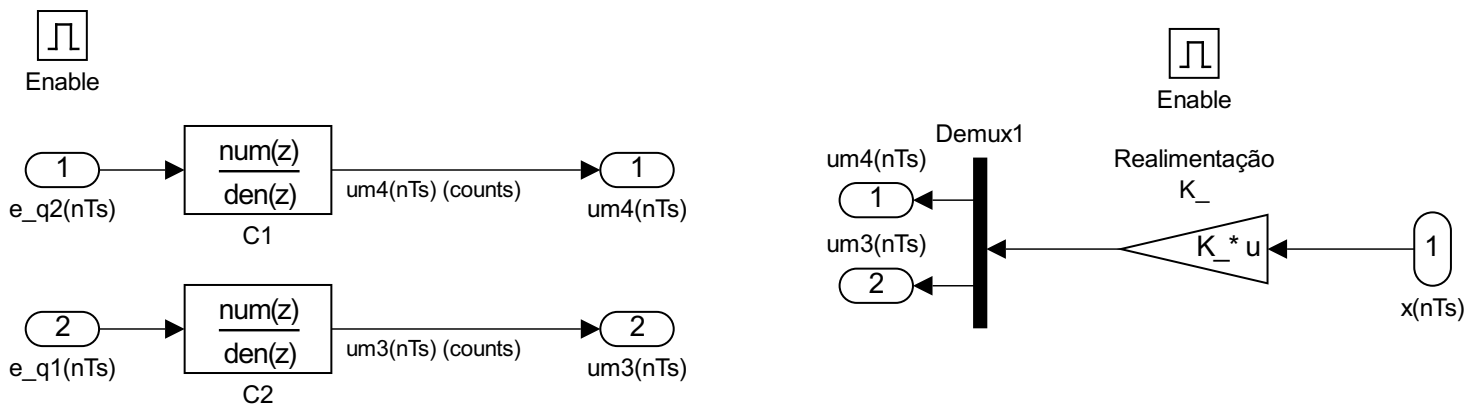

(c) Subsistema "Controle PI de velocidade do disco" utilizada em todos os sistemas

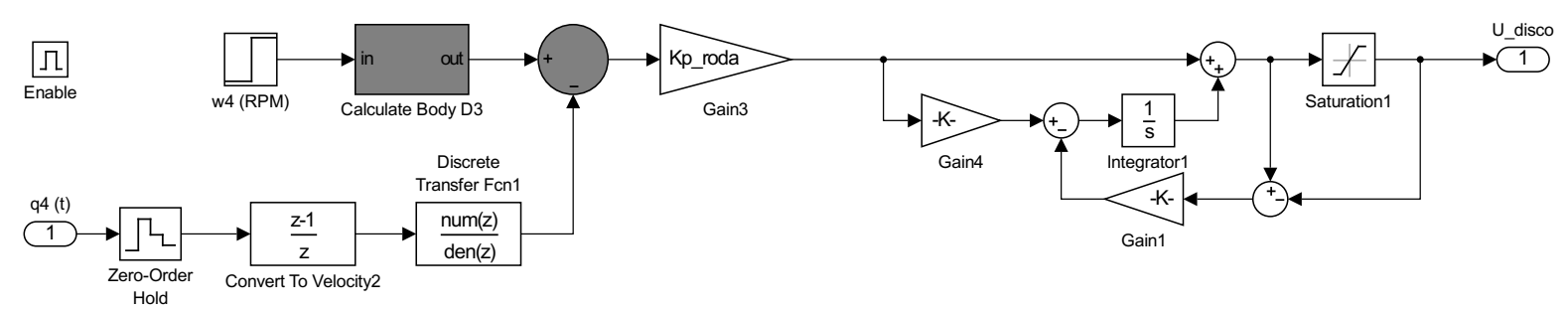

Fonte: Autor.

Figura 67: Algoritmo do bloco Matlab Function com as equações discretas do controlador LQR

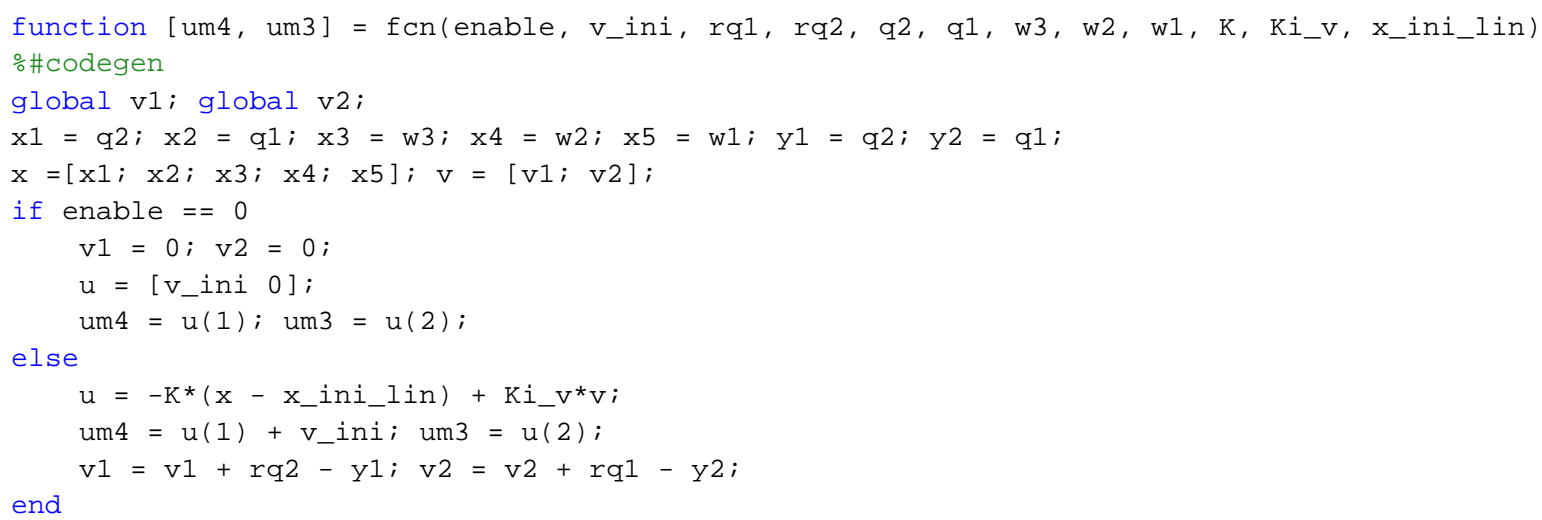

Fonte: Autor. 


\section{APÊNDICE J - ALGORITMOS CRIADOS NO MATLAB ${ }^{\circledR}$}

\section{J.1 Algoritmo criado para o controle PID}

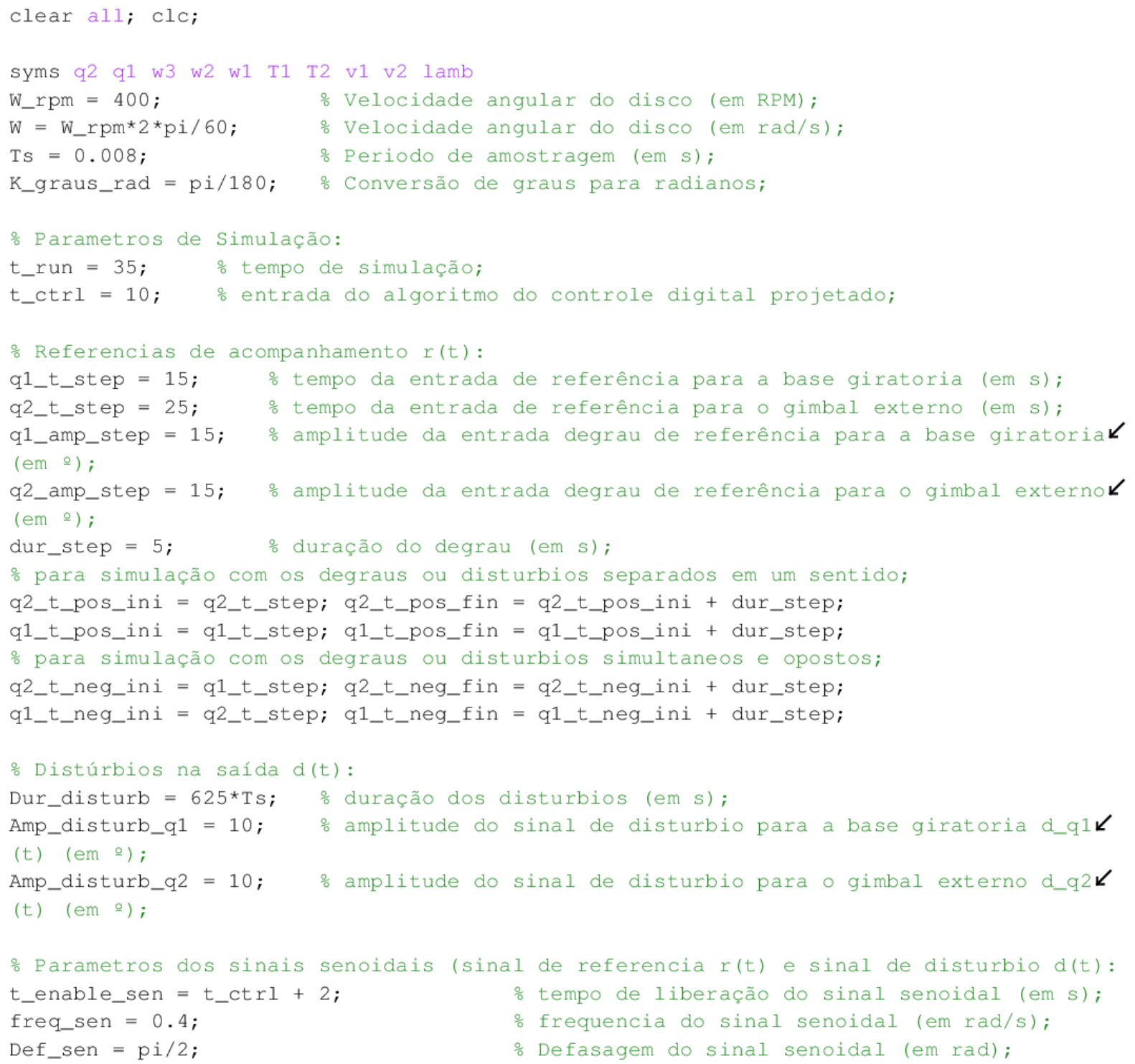




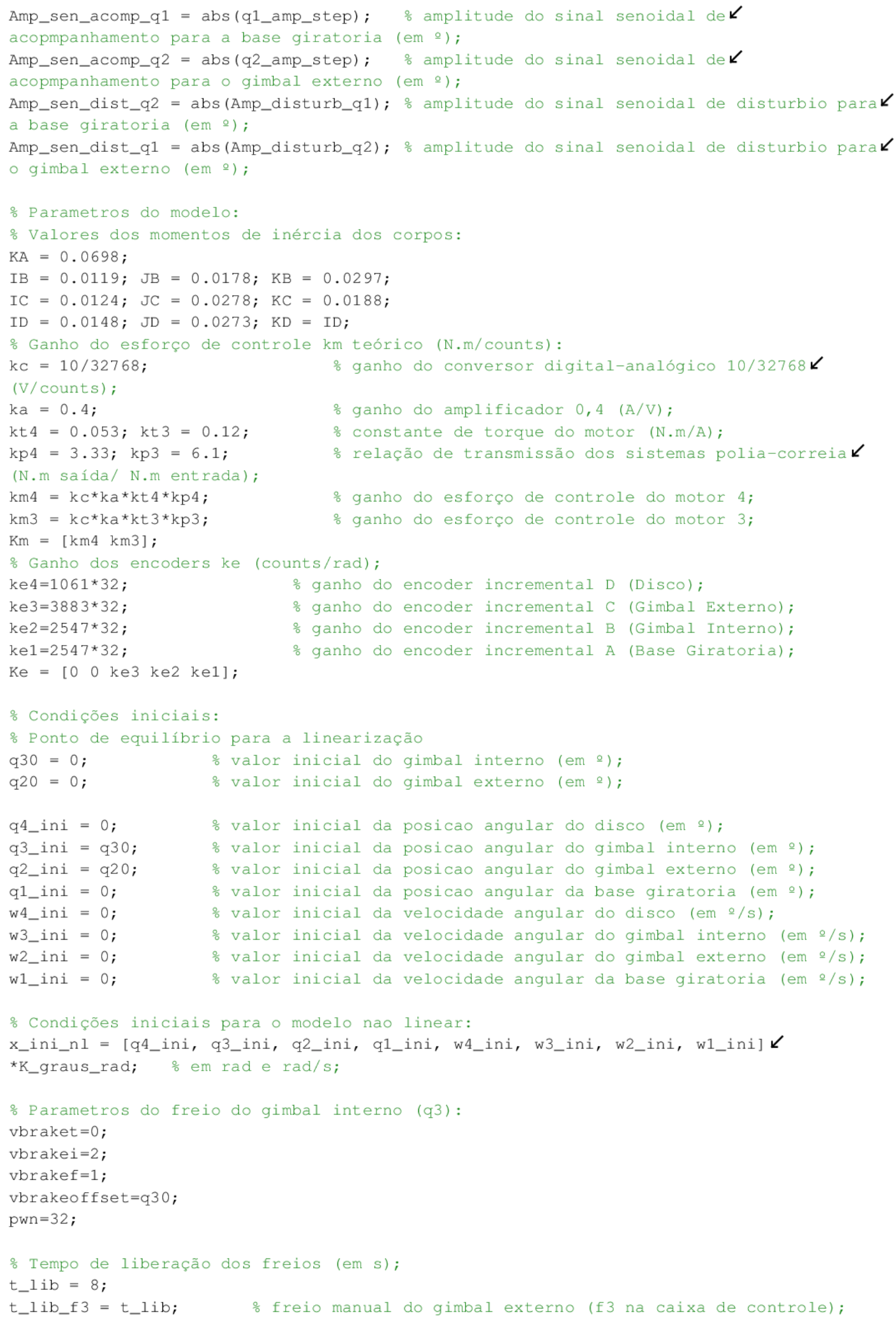




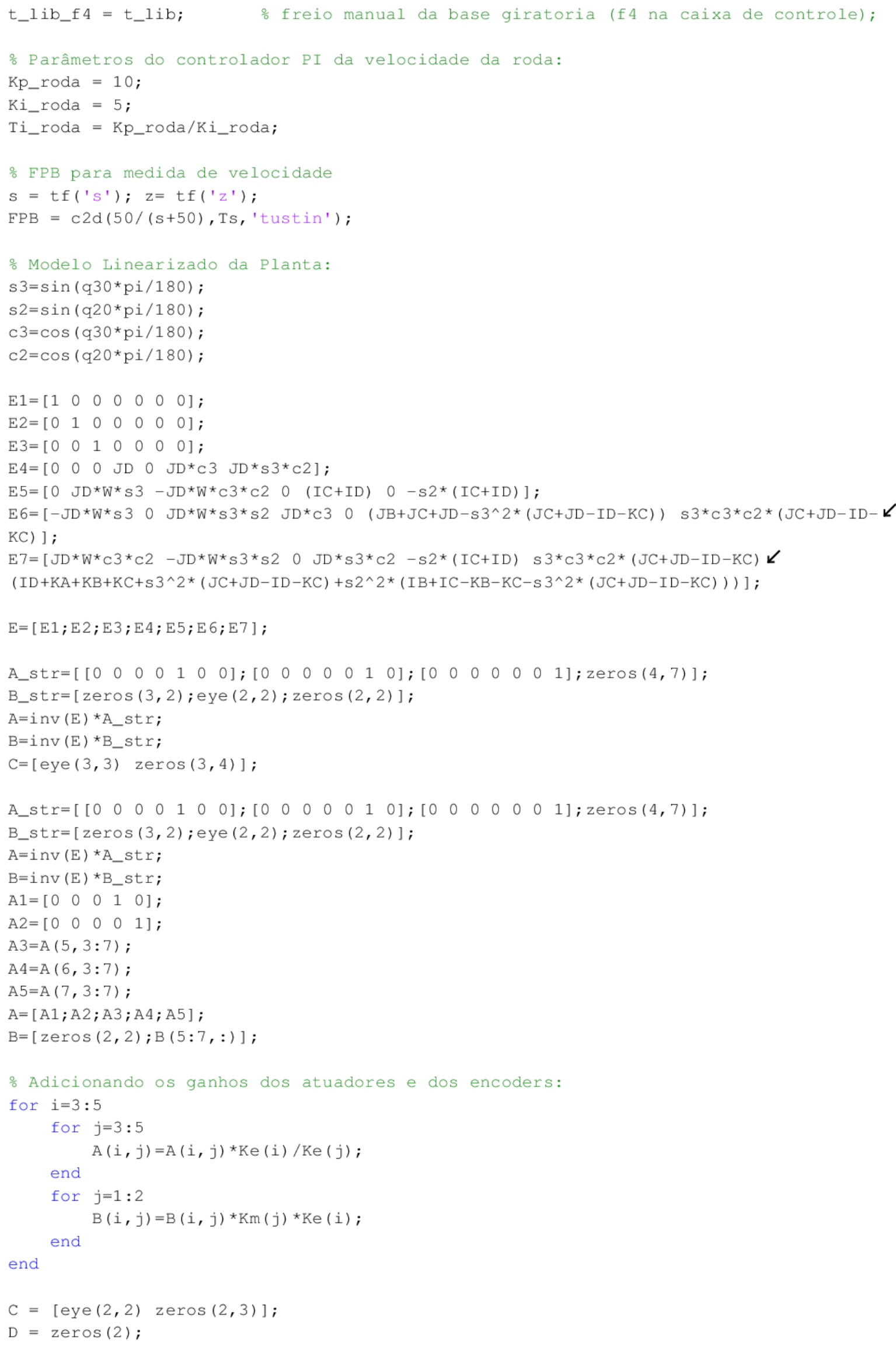




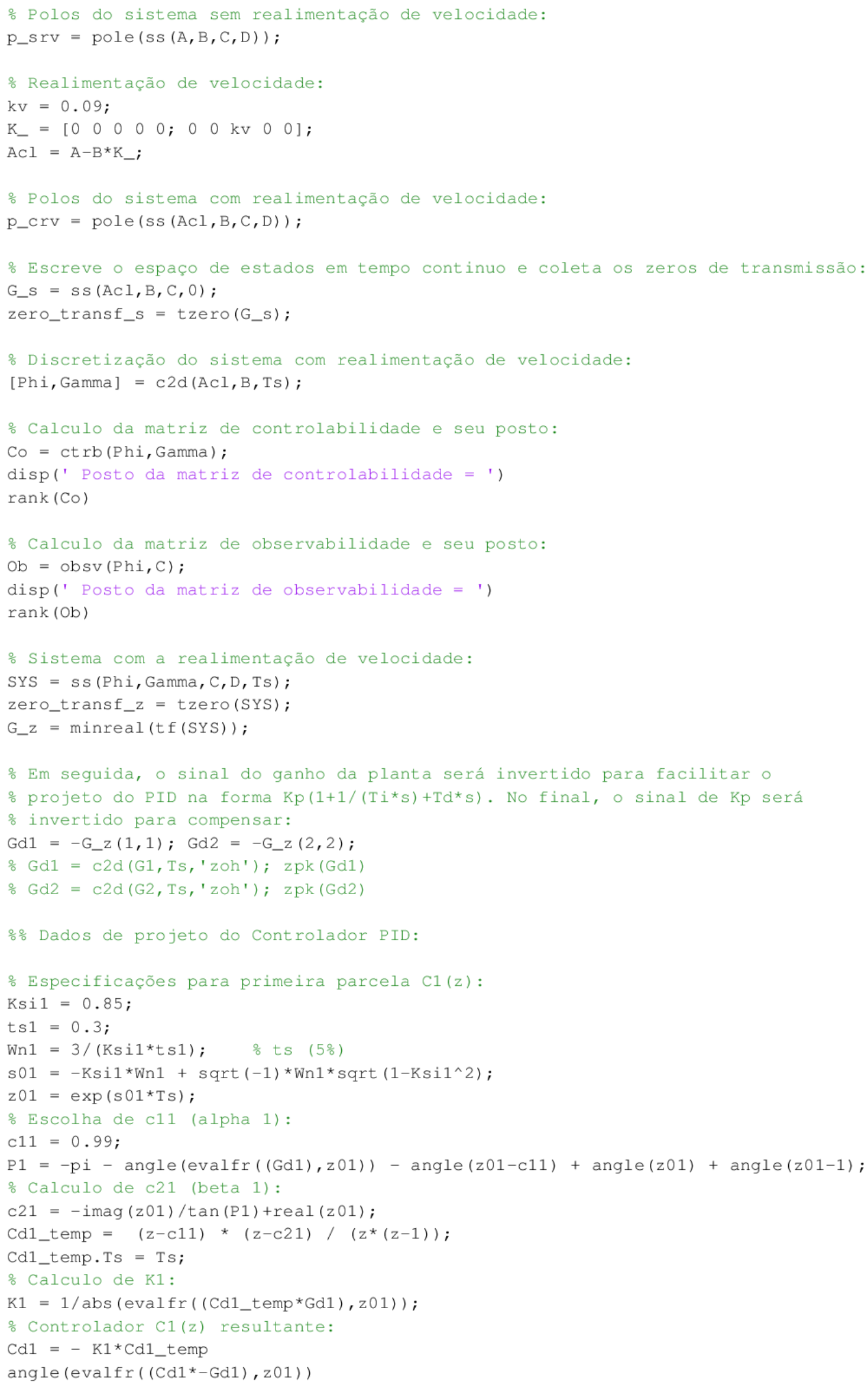




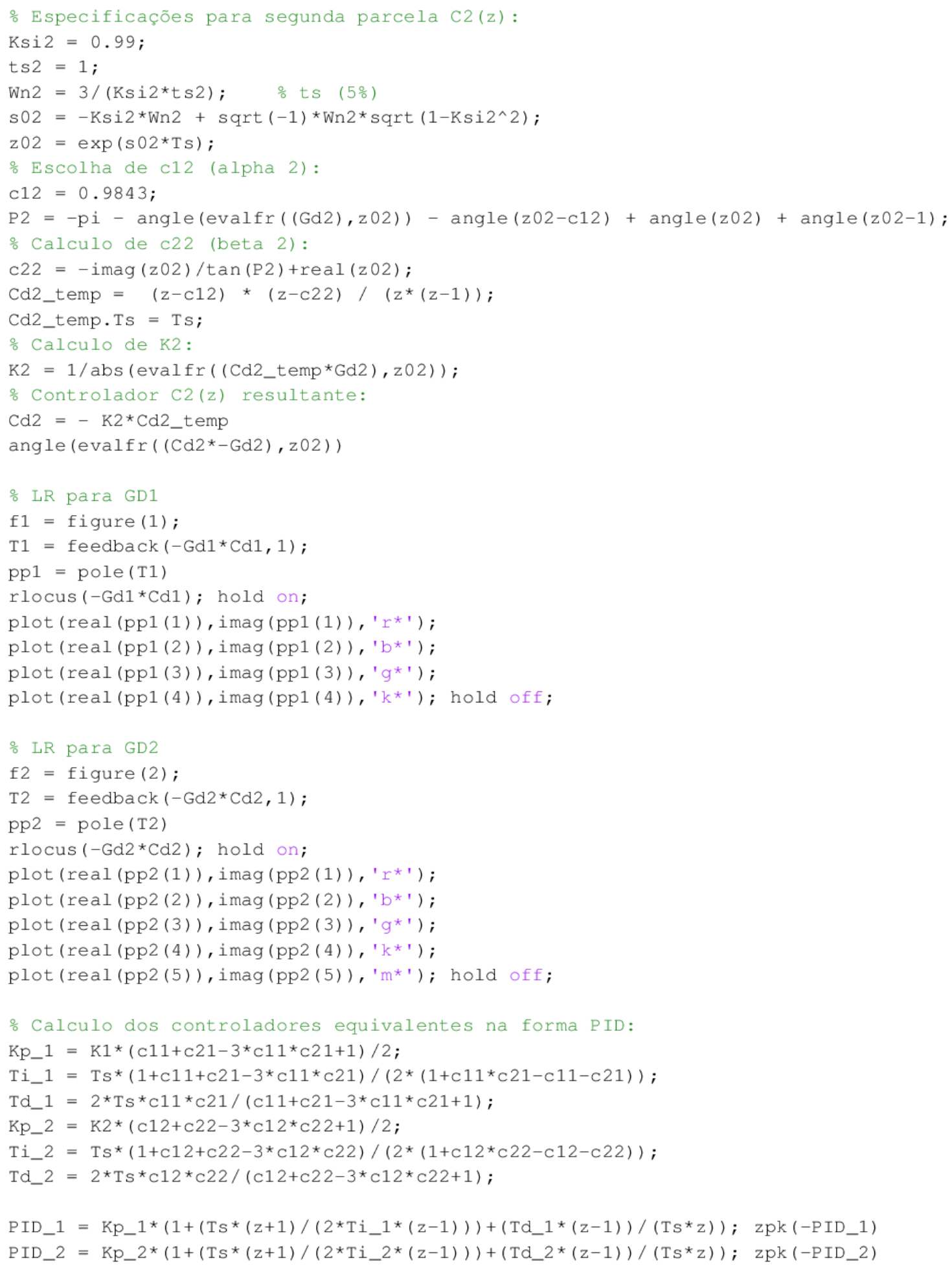




\section{J.2 Algoritmo criado para o controle LQR}

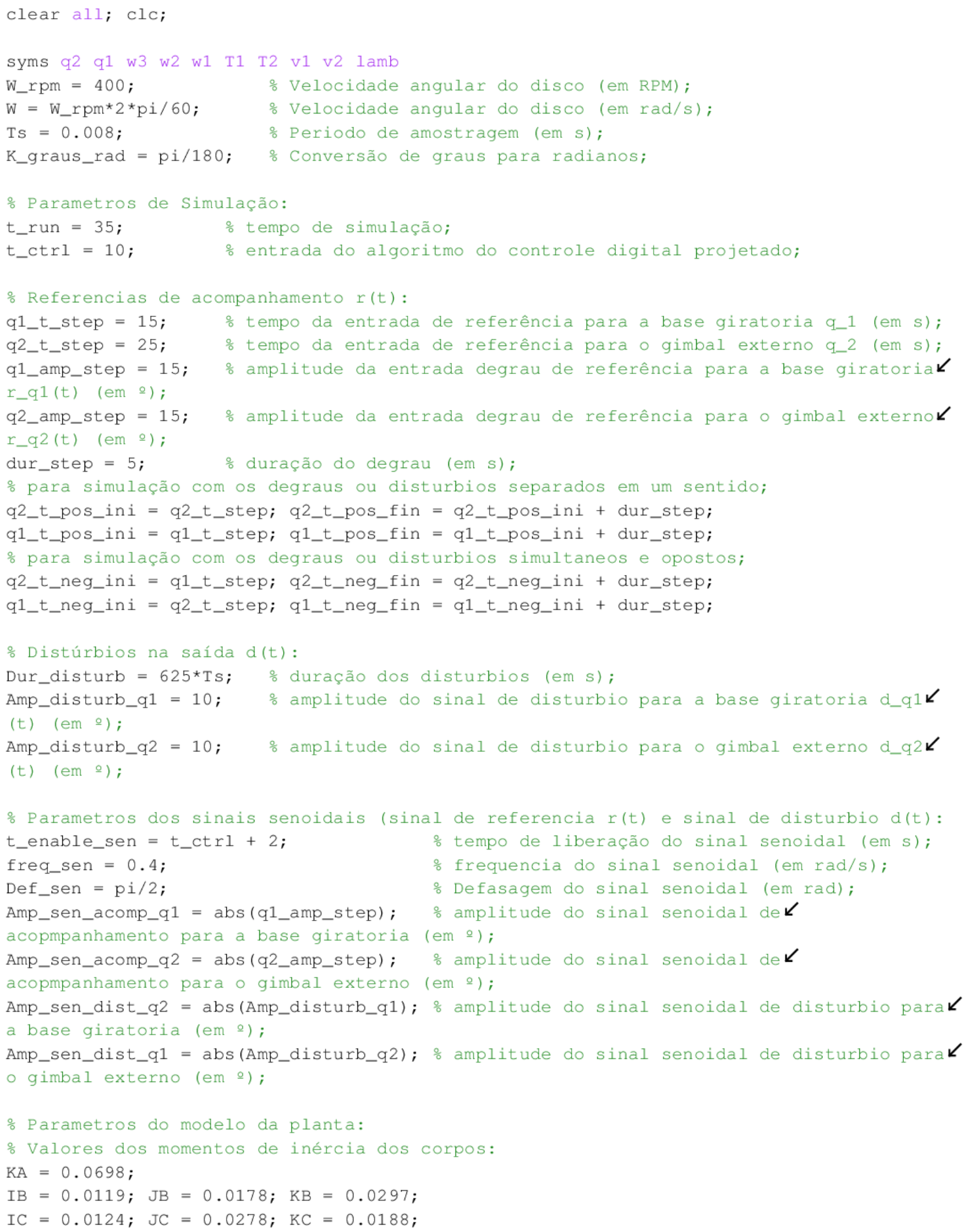




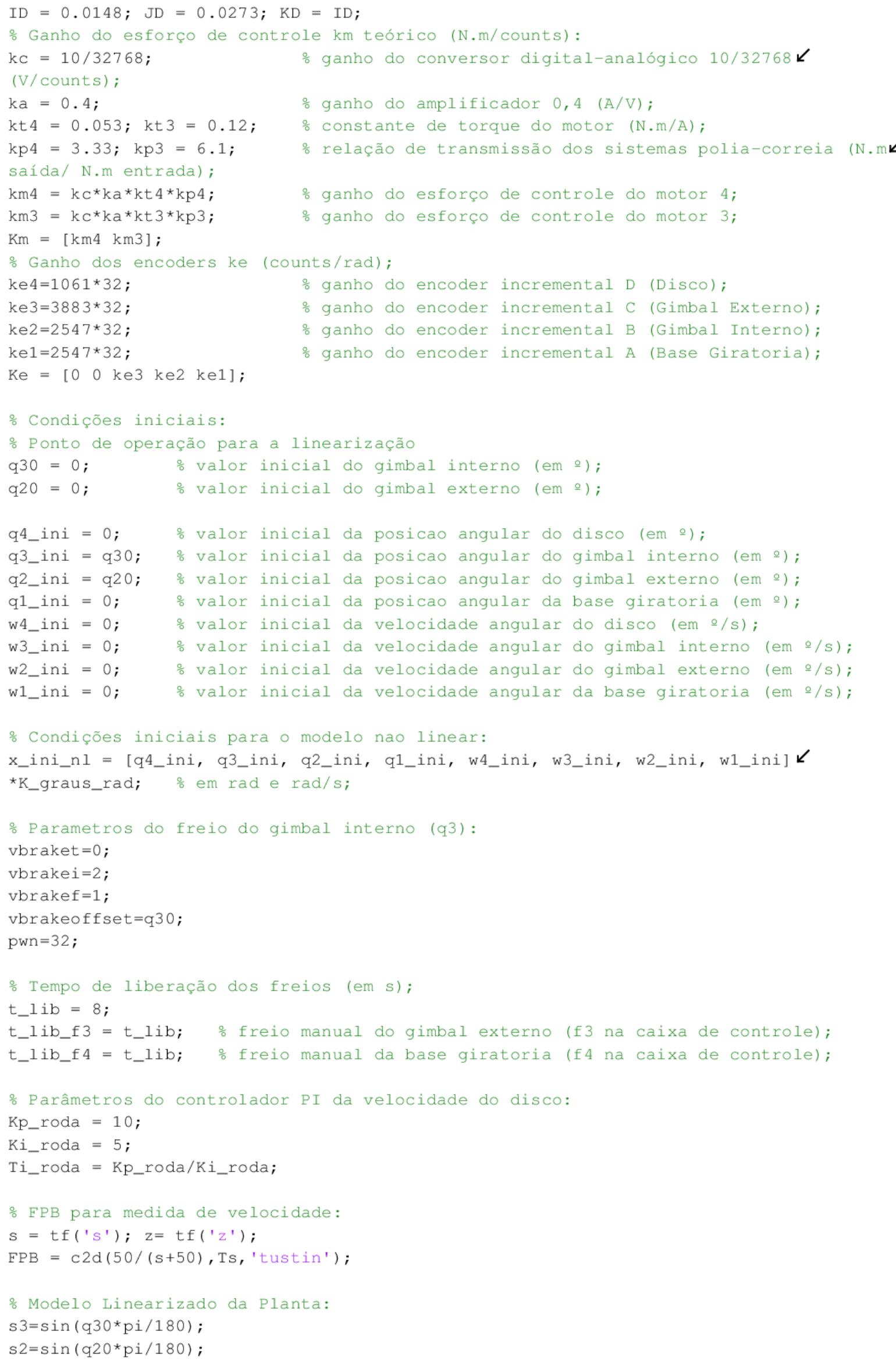




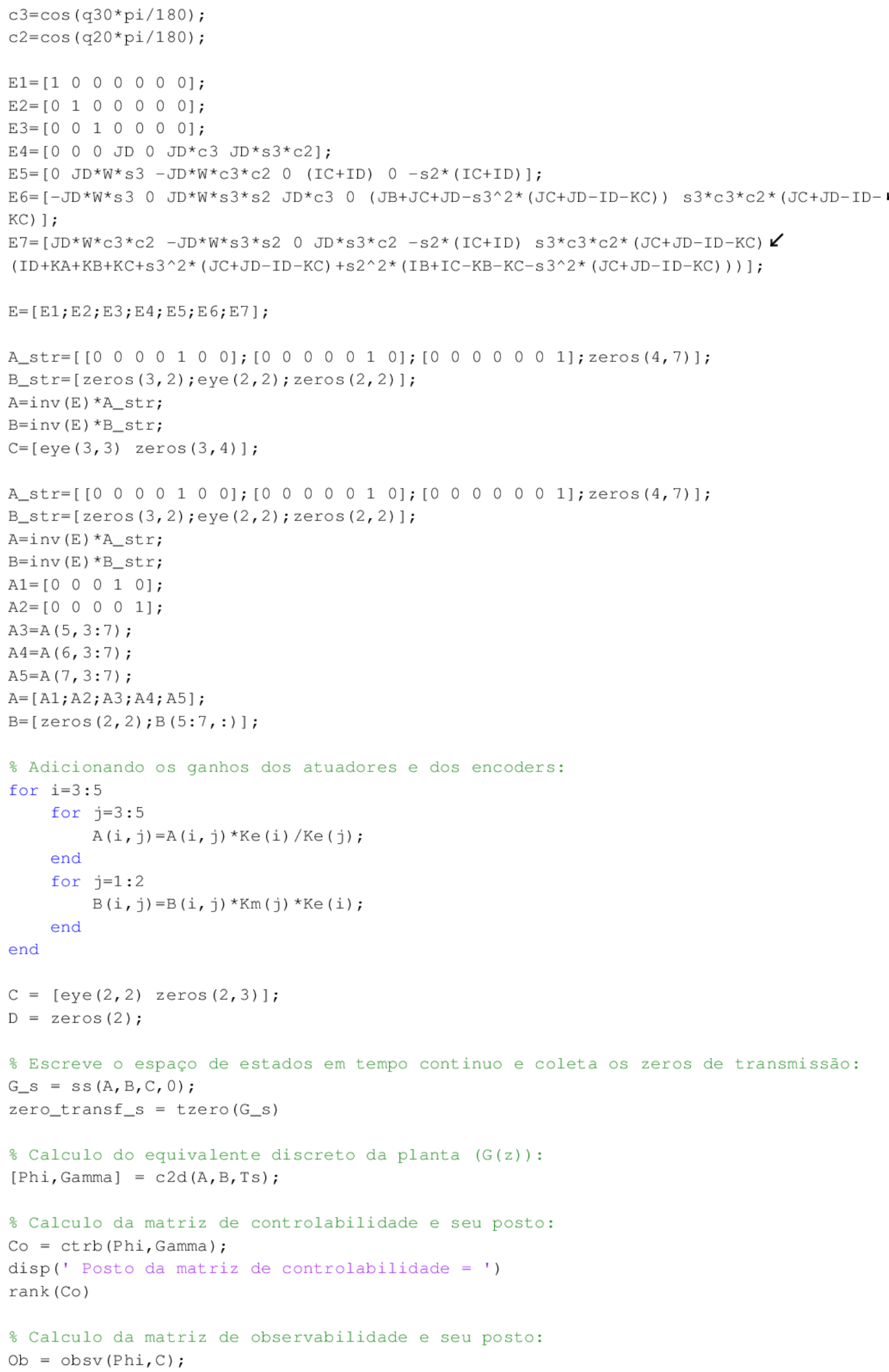




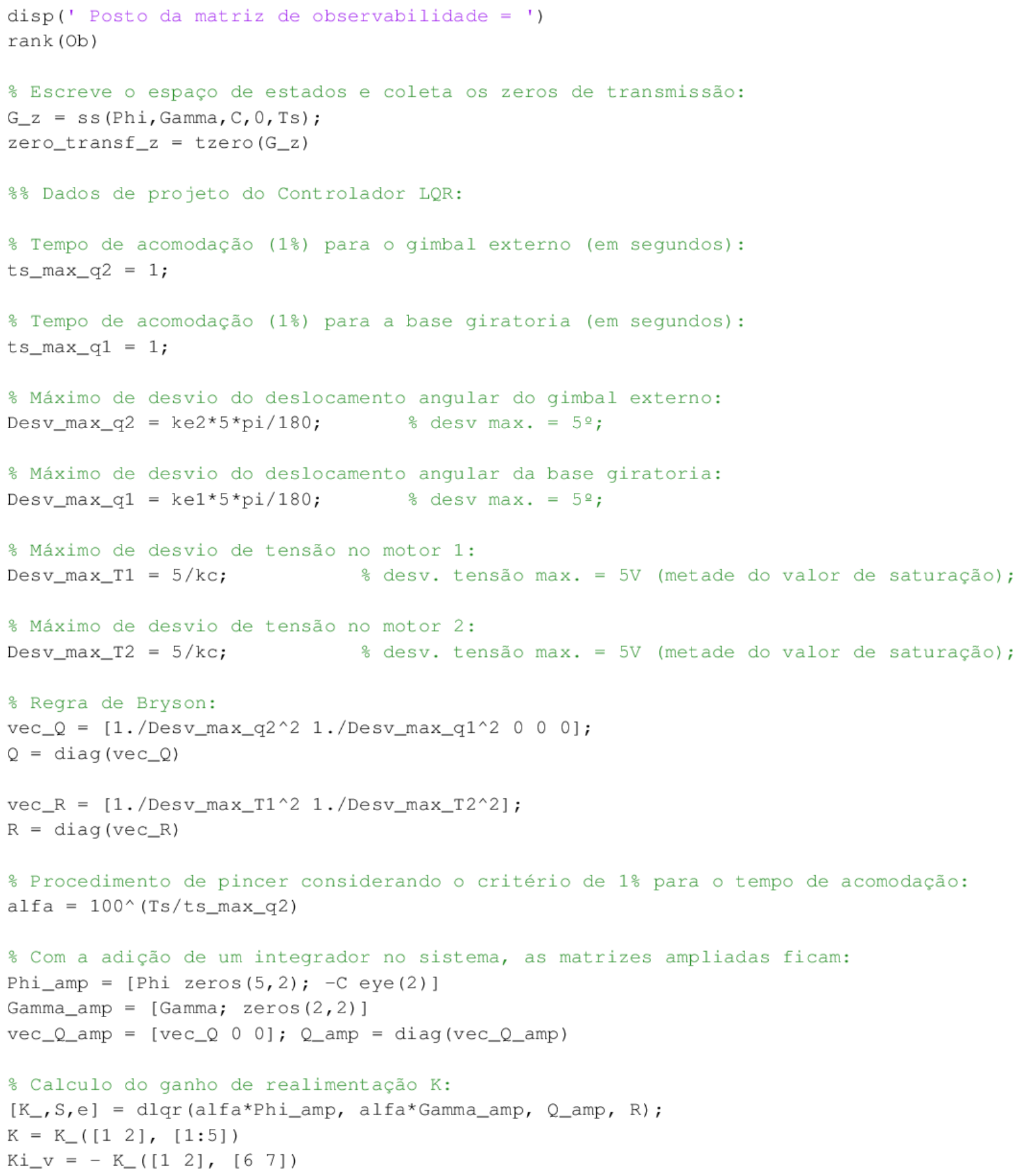

